\title{
Implementation of a Self-Replicating Universal Turing Machine
}

THÈSE № 2457 (2001)

\author{
Présentée au Département d'informatique \\ ÉCOLE POLYTECHNIQUE FÉDÉRALE DE LAUSANNE
}

pour l'obtention du grade de Docteur ès sciences techniques

par

\section{Héctor Fabio Restrepo-García}

Ingeniero Electricista, Magister en Automática, Universidad del Valle, Cali, Colombie, de nationalité colombienne

acceptée sur proposition du jury:

$\begin{array}{ll}\text { Prof. D. Mange } & \text { directeur de thèse } \\ \text { Prof. R. D. Hersch } & \text { président du jury } \\ \text { Prof. G. Coray } & \text { rapporteur } \\ \text { Dr. P. Marchal } & \text { rapporteur } \\ \text { Prof. E. Sanchez } & \text { rapporteur } \\ \text { Prof. A. Tyrrell } & \text { rapporteur }\end{array}$

Lausanne, EPFL 

This work is dedicated to the memory of my father, to my mother, to my wife and to my two child -Daniela and Pablo-who, despite their few years, have more to teach me than I have to teach them. 



\section{Abstract}

The field of bio-inspired digital hardware was pioneered by John von Neumann. A gifted mathematician and one of the leading figures in the development of the field of computer engineering, von Neumann dedicated the final years of his life on what he called the theory of automata. His research, which was unfortunately interrupted by his untimely death in 1957, was inspired by the parallel between artificial automata, of which the paramount example are computers, and natural automata, such as the nervous system, evolving organisms, etc.

Through his theory of automata, von Neumann conceived a set of machines capable of many of the same feats as biological systems: evolution, learning, self-repair, selfreplication, etc. At the core of his approach was the development of self-replicating machines, that is, machines capable of producing identical copies of themselves.

This thesis is part of a more general research project, called Embryonics (a contraction of the words embryonic electronics), which aims at establishing a bridge between the world of biology and that of electronics, and in particular between molecular biology and digital systems. One of the main goals of the Embryonics project is to determine if, given modern technology, Von Neumann's dream of a self-replicating machine can be realized in hardware.

Thus, inspired by von Neumann ideas, the first goal of this thesis was to realize, using the Embryonics architecture, the first hardware implementation of a machine capable of self-replication and exhibiting the property of universal computation, i.e., a universal Turing machine. The hardware architecture of the machine consists of a multicellular two-dimensional array of artificial cells (MICTREE cells) developed in our laboratory.

These MICTREE cells, a new kind of coarse-grained field-programmable gate array, are used for the implementation of multicellular artificial organisms with biologicallike properties, i.e., capable of self-repair and self-replication. But the design of the software programs defining the behavior of the MICTREE cells is difficult and until now, had to be realized by hand, an approach that often led to errors in the software description. These hard-to-find errors increased considerably the design time of an Embryonics system.

Thus, the second goal of this thesis was to develop a programming methodology to deal with complex applications in the Embryonics project. We present, in order to demon- 
strate this methodology, a detailed description of the implementation of some examples (from simple to complex), all based on a top-down design process characterized by the systematic decomposition of a complete microprogram (an artificial genome) into a set of smaller sub-microprograms, by the systematic construction and optimization of binary decision trees and diagrams, and by a rigorous organization of these sub-microprograms according to the demands of the Embryonics architecture. We finally developed a coherent family of software tools (MIC Editor, MIC Compiler, Visual BDD, MIC Sim, and a serial loader) that have been integrated into a single graphical design environment in order to achieve versatility and user-friendliness. 


\section{Résumé}

John von Neumann, un mathématicien exceptionnel et l'un des pères de l'ordinateur, a consacré les dernières années de son existence à la théorie des automates. Ses recherches, malheureusement interrompues par son décès en 1957, ont été inspirées par l'analogie existant entre les automates artificiels, dont l'exemple principal est l'ordinateur, et les automates naturels, tels que le système nerveux, les organismes évolutifs, etc.

Grâce à la théorie des automates, von Neumann a conçu un ensemble de machines munies de caractéristiques semblables à celles des systèmes biologiques : évolution, apprentissage, auto-réplication, auto-réparation, etc. Le développement de machines autoréplicatives, c'est-à-dire capables de produire des copies d'elles-mêmes, est au cœur de son approche.

Cette thèse fait partie d'un projet de recherche plus général appelé Embryonique (une contraction des mots embryologie électronique) qui vise à jeter un pont entre le monde de la biologie et celui de l'électronique, et en particulier entre la biologie moléculaire et les systèmes digitaux. Un des buts principaux du projet Embryonique est de déterminer si, avec la technologie moderne, le rêve de von Neumann, -la construction d'une machine auto-réplicative_ - peut être réalisé à l'aide du matériel actuel.

Sur la base des idées de von Neumann, le but principal de cette thèse est de réaliser, grâce à l'architecture embryonique, la première implémentation matérielle d'une machine capable d'auto-réplication et de calculation universelle, c'est-à-dire une machine de Turing universelle. L'architecture matérielle se compose d'un réseau bidimensionnel multicellulaire artificiel (cellules MICTREE) développé dans notre laboratoire.

Les cellules MICTREE, un nouveau type de FPGA (Field-Programmable Gate Array) à grain grossier, sont utilisées pour la mise en œuvre d'organismes multicellulaires artificiels ayant des propriétés inspirées par la Nature, c'est-à-dire capables d'auto-réparation et d'auto-réplication. Mais la conception des programmes définissant le comportement de ces cellules est difficile et a dû être réalisée, jusqu'ici, à la main. Cette approche conduit souvent à des erreurs dans l'écriture du logiciel. Ces erreurs sont très difficiles à corriger, ce qui augmente le temps de conception.

Le deuxième but de cette thèse est le développement d'une méthodologie de programmation apte à maîtriser des applications complexes du projet Embryonique. Pour démontrer cette méthodologie, nous présentons la description détaillée de plusieurs ex- 
emples, du plus simple au plus compliqué; tous ces exemples sont calculés à l'aide d'une méthode descendante (top-down) caracterisée par une décomposition du microprogramme complet (notre génome artificiel) en un ensemble de sous-microprogrammes, par la construction et l'optimisation systématiques d'arbres ou de diagrammes de décision binaire, et par une organisation rigoureuse des sous-microprogrammes particulièrement adaptée à l'architecture Embryonique. Nous présentons enfin les différents outils logiciels (MIC Editor, MIC Compiler, Visual BDD, MIC Sim et un chargeur série) qui ont été intégrés dans un environnement graphique afin d'obtenir souplesse et convivialité. 


\section{Acknowledgments}

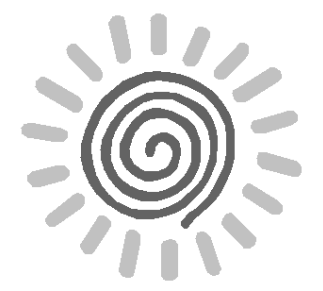

On the long path to realize this work, a lot of steps have required the support and the teaching of many people. Unfortunately, it would be impossible to individually thank all those who deserve it. Therefore, to those whose valuable comments and help are not explicitly acknowledged, my thanks and apologies.

I hesitated a lot before deciding to write down the words composing the next paragraph, but I think that it is the best way to honor my father's memory and all the things he and my mother did for me and my brother.

I was born in my grandmother's house, in a little room that was, from that moment, shared with my parents and one year later with my brother. Thus, for many years we shared not only the same room, but also a lot of wonderful moments, and even if our economic possibilities were very limited, those days were full of love and happiness. My father only did two years of elementary school but he was a passionate reader and an intelligent person. He was always astonished by technology and he always astonished and inspired me. My mother helped me take my first steps in life, taught me the first syllables, the first words, and taught me to read and write. I thank them for those days, for all their lessons, for all the sacrifices they made to grow us up and send us to school, and for giving us almost all the necessities of life.

Thanks to all my relatives, for their warm support, for their sharing, for their helping us in very difficult economic days, even when they didn't have enough for themselves.

I particularly want to thank my wife Graciela for her love, for the trust she placed in me, for her support, patience, and understanding during my work. And finally for every thing we have shared during the last fifteen years.

I want to thank my daugther Daniela, whom I call tenderly "mi solecito de amor" (my little sun of love). She taught, and is still teaching, me a lot of things, she showed me that children are true researchers with their everyday wondering about simple, day-to-day, but important things: Daddy, why is the moon always following us? How many moons are there on the sky? Who was the mother of the first man on the earth and how did she come here? Why and when, when we grow up, do we lose this everyday wondering about simple and wonderful things? Finally, I want to thank Daniela for telling me her dreams.

I want to thank my son Pablo, "Pablito" (little Pablo) as I call him. He was born in the last year of my thesis, so as you can imagine I can't thank him for the time he left me 
to work on my thesis. Rather, I thank him for the splendid and wonderful experiences we shared from the early days in his mother's womb until now, when each of his smiles or his grabbing and squeezing my finger with his strong, sweet, and little hand is my greatest and best present.

I am profoundly grateful to Eduardo Sanchez and his family, Martha, María Isabel, and Pilar Cristina, for their warm support, their friendship, and for the pleasant moments shared. Eduardo has been able to manage his research without forgetting that human relationships are essential. He always figured out a solution for every problem without losing his great sense of humour. I want to thank him for his unconditional and invaluable support, for his sincere friendship, and his always opportune and heartfelt help and guidance.

Thanks to Daniel Mange, my thesis director. I thank him not only for his mentoring, encouragement, and hard work correcting and improving various drafts of my manuscript, but also for being a friend, an understanding person, and the soul of the Embryonics project. I finally thank him for the trust he has placed in me.

I would like to thank the members of the Ph.D. thesis committee, namely Prof. R. D. Hersch, Prof. G. Coray, Prof. A. Tyrrell, Prof. E. Sanchez, and Dr. P. Marchal for accepting to read and evaluate my work and for providing valuable suggestions and comments.

I take this opportunity to thank all those members of the laboratory who made it possible for me to finish this thesis and made my stay at Logic Systems Laboratory (LSL) a pleasant one.

I am profoundly grateful to Gianluca Tempesti for the time and effort spent proofreading this manuscript, and for his valuable suggestions and comments.

I am very grateful to Marlyse Taric, our lab's charming secretary, for all her invaluable help, discussions about different and interesting subjects, and her friendliness.

I thank André Badertscher, colleague, soldering wizard, wildlife expert, and official photograph of the lab. I am very grateful to him for his friendliness, his concern about nature, and his practical and helpful advice in different areas.

Thanks to my different officemates during these last years: Maxime Goeke, JeanMichel Puiatti, Jean-Luc Beuchat, Jacques-Olivier Haenni, Christof Teuscher, and Fabien Vannel. Them were all always ready to help me in different situations and shared with me a lot of good things, from wonderful Jacques Brel songs, Italian and Spanish music, classical music, XBlast, Latex and VHDL advice, and French grammar assistance.

Thanks to all my others colleagues in the lab: Gianluca Tempesti, Dominik Madon, Yann Thoma, Mathieu Capcarrere, Enrico Petraglio, Jacques Zahnd, André Stauffer, Ralph Hoffmann, Moshe Sipper, Carlos Andrés Peña, Isis Giraldo, and José Diego de la Cruz. I am very grateful to all of them for contributing to make my stay at the LSL so pleasant and enriching. 
Thanks to Walter Pineda, Antonio Restrepo, Andrés Pérez, Carlos Andrés Peña, and José Luis Bermúdez for their friendship, useful discussions about their work, my work, and a lot of other technical and social problems.

I am profoundly grateful to all my Colombian friends. They have been like a second family for me. I thank them for all the interesting dinner-time discussions about our country, Colombia, science, religion, politics, and economy, as well as for all the experiences and moments shared in parties, Christmases, births, birthdays, etc., all of which were particularly enriching for me.

I am very grateful to Monika, Jerry, and Dino for their warm welcome in their home in California and for the pleasant moments shared. Thanks to Monika for her sincere friendship and unconditional support.

I want to thanks Pierre Marchal, and Pascal Nussbaum of the Centre Suisse d'Electronique et Microtechnique, and Arnaud Tisserand of the Laboratoire de l'Informatique du Parallélisme de l'Ecole Normale Supérieure de Lyon. I would like to thank in particular Pierre Marchal for his friendly and warm smile, his unconditional friendship and his understanding.

I thank the Commission fédérale des bourses pour étudiants étrangers for its financial support during my first years in Switzerland, COLCIENCIAS, the Centre Swiss d'Electronique et de Microtechnique (CSEM), and the EPFL for supporting my work. 



\section{Table of Contents}

$\begin{array}{lll}\text { Abstract } & \mathbf{v}\end{array}$

Résumé vii

$\begin{array}{ll}\text { Acknowledgments } & \text { ix }\end{array}$

1 Introduction 1

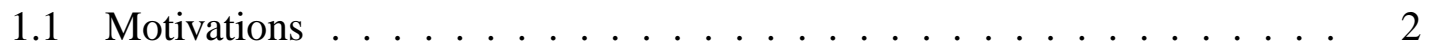

1.2 The Embryonics project . . . . . . . . . . . . . . . . 4

1.2.1 Overview of the project . . . . . . . . . . 4

1.2.2 The POE model . . . . . . . . . . . . . . . . 5

1.2.3 Phylogeny ....................... 5

1.2 .4 Epigenesis . . . . . . . . . . . . . . 6

1.2 .5 Ontogeny ....................... 7

1.3 Outline of the thesis . . . . . . . . . . . . . 8

2 History of Self-Replicating Machines $\quad 11$

2.1 Self-replicating automata . . . . . . . . . . . . . . 12

2.1.1 Von Neumann's self-replicating machines . . . . . . . . . . . 13

2.1.2 Von Neumann's cellular automaton . . . . . . . . . . 13

2.1.3 Von Neumann's successors . . . . . . . . . . . . . . . . 15

2.2 Self-replicating loops $\ldots \ldots \ldots \ldots$. . . . . . . . . . . . . 17

2.3 Self-replicating loops with computing capabilities . . . . . . . . . . . . . 19 
2.4 Self-replicating loops with universal computing capabilities . . . . . . . . . . . . . . . . . . 20

2.5 Self-replication of Turing machines on a multicellular array: The Embryonics approach . . . . . . . . . . . . . . . 21

3 The MICTREE Cell 23

3.1 General description . . . . . . . . . . . . . . . . . . 24

3.2 MICTREE features . . . . . . . . . . . . . . . 26

3.2 .1 Self-repair . . . . . . . . . . . . . . 27

3.2 .2 Self-replication . . . . . . . . . . . . . . 28

3.3 Example 1: A random number generator . . . . . . . . . . 28

3.3.1 Description . . . . . . . . . . . . . . . . . 29

3.3.2 Computing the gene for rule $90 \ldots \ldots 31$

3.3.3 Computing the gene for rule $150 \ldots \ldots 33$

3.3.4 Computing the operational part of the genome . . . . . . . . . . 34

3.3.5 Computing the coordinates . . . . . . . . . . . . 35

3.3.6 Physical configuration . . . . . . . . . . . . 37

3.3.7 Global configuration . . . . . . . . . . . . . . 38

3.3 .8 Local configuration . . . . . . . . . . . . . . . . 38

3.3.9 Microprogram of the complete genome . . . . . . . . . . . 40

3.4 Example 2: A 2-by-n hybrid cellular automaton . . . . . . . . . . . . 42

3.4.1 Genes, coordinates, and genome . . . . . . . . . . . . 43

3.4.2 Configurations and microprogram . . . . . . . . . . . 46

3.5 Software tools . . . . . . . . . . . . . . . . . . 50

3.5.1 MIC Editor and MIC Compiler . . . . . . . . . . . . 50

3.5 .2 Visual BDD . . . . . . . . . . . . . . 50

3.5 .3 MIC $\operatorname{Sim} \ldots \ldots \ldots \ldots \ldots$

3.5.4 Visual MICTREE design environment . . . . . . . . . . . . . . 51

3.6 Reliability analysis of the Embryonics architecture . . . . . . . . . 51 


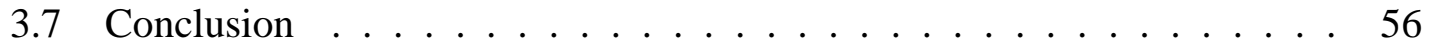

4 Specialized Turing Machines

$4.1 \quad$ Specialized Turing machines $\ldots \ldots \ldots$. . . . . . . . . . . 59

4.2 A parenthesis checker . . . . . . . . . . . . . . . 61

4.3 Self-replication of specialized Turing machines on a multicellular array: the Embryonics approach . . . . . . . . . . . . . . . . . . 63

4.3.1 Embryonics features . . . . . . . . . . . . . 63

4.3.2 Self-repair and self-replication . . . . . . . . . . . . 65

4.4 Detailed calculation of a Turing machine . . . . . . . . . . . . . . 67

4.4 .1 Specifications . . . . . . . . . . . . . 67

4.4.2 Coordinates and initial conditions . . . . . . . . . . . . . 69

4.4 .3 Genes and genome . . . . . . . . . . . . 71

4.4.4 Configurations and microprogram . . . . . . . . . . . . 77

4.5 Conclusion . . . . . . . . . . . . . . . . 80

5 Universal Turing Machine $\quad 83$

5.1 The universal Turing machine $\ldots \ldots \ldots . \ldots . \ldots . \ldots$

5.2 Self-replication of a universal Turing machine on a multicellular array . . 84

5.2.1 Multicellular architecture of a universal Turing machine . . . . . 84

5.2.2 An application: A binary counter . . . . . . . . . . 85

5.2.3 An ideal architecture for the universal Turing machine . . . . . 86

5.2.4 An actual implementation of the UTM for the binary counter example ........................ 88

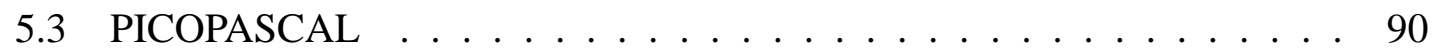

5.3.1 The PICOPASCAL language . . . . . . . . . . . . . . 90

5.3.2 PICOPASCALINE: an interpreter for the PICOPASCAL language . . . . . . . . . . . . . 92

5.4 Detailed calculation of a universal Turing machine $\ldots \ldots \ldots$

5.4.1 Computing the coordinates . . . . . . . . . . . . . 98 
5.4.2 Computing the initial conditions . . . . . . . . . . . . 98

5.4.3 Computing the operative genome . . . . . . . . . . . . . 99

5.4.4 Computing the program tape genes . . . . . . . . . . 103

5.4.5 Computing the stack genes . . . . . . . . . . . . 105

5.4.6 Computing the $(Q, S)$ REGISTER genes . . . . . . . . . . . . . 109

5.4.7 Computing the $(Q L 3: 0, Q C, Q R 0: 3)$ REGISTER genes . . . . . . 112

5.4 .8 Global configuration . . . . . . . . . . . . . 117

5.4 .9 Local configuration . . . . . . . . . . . . . . . . . 118

5.4 .10 Final microprogram . . . . . . . . . . . . . 122

5.5 Experiment and discussion . . . . . . . . . . . . . . . . . . . . 124

5.6 Conclusion . . . . . . . . . . . . . . . . . . 127

6 Software Tools $\quad 129$

6.1 MIC Editor and MIC Compiler . . . . . . . . . . . . . . . . . . 129

6.2 Visual BDD . . . . . . . . . . . . . . . . . . 130

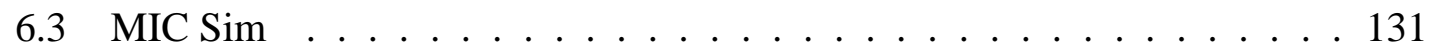

6.3.1 Simulation engine . . . . . . . . . . . . . 132

6.3.2 MICTREE model . . . . . . . . . . . . . . . . . 133

6.3 .3 Input/output cell model . . . . . . . . . . . . . . . . . 135

6.3.4 Cellular network model . . . . . . . . . . . . 135

6.3.5 MIC Sim implementation . . . . . . . . . . . . . 135

6.4 Visual MICTREE design environment . . . . . . . . . . . . . . 138

6.5 2-by-n hybrid cellular automaton example . . . . . . . . . . . . . 139

6.6 Conclusion . . . . . . . . . . . . . . . . . . . 143

7 Conclusions $\quad 145$

7.1 Original contributions . . . . . . . . . . . . . . . . 146

7.2 Problems and issues . . . . . . . . . . . . . . . . . . 147

7.3 The future of Embryonics . . . . . . . . . . . . . . . . . . . . 148 
7.3.1 The BioWatch on the BioWall's computing substrate . . . . . . 149

7.3.2 Towards Bio-Inspired Computing Tissues . . . . . . . . . . . . 150

7.3.3 Towards POEtic Machines . . . . . . . . . . . . . . . 150

A Cellule MICTREE : description et utilisation 153

A.1 Interfaçage du biodule $601 \ldots \ldots$. . . . . . . . . . . . . 154

A.1.1 Généralités . . . . . . . . . . . . . . . . . 154

A.1.2 Configuration classique . . . . . . . . . . . . 156

A.1.3 Câblage de l'horloge $(C K) \ldots \ldots . \ldots . \ldots 157$

A.1.4 Câblage du signal de remise à zéro $(C L R) \quad \ldots . . . .157$

A.1.5 Câblage pour le transfert du génome $(D I N$ et $D O U T) \quad \ldots . .157$

A.1.6 Câblage des signaux de coordonnées $(W X, X, S Y$ et $Y) \quad \ldots 159$

A.1.7 Câblage du signal de suicide commun $(B Y P) \quad \ldots . \ldots .159$

A.1.8 Câblage du signal de synchronisation global $(G) \quad \ldots \ldots$

A.1.9 Connexion avec le MacIntosh et son interface LSLParallelIO . . . 160

A.1.10 Connexion avec le MacIntosh et son interface série LoaderSer . . 161

A.2 Fonctionnement interne . . . . . . . . . . . . . . . . 162

A.2.1 Caractéristiques générales . . . . . . . . . . . . . 162

A.2.2 Machine de décision binaire . . . . . . . . . . . . . . 162

A.2.3 Routeur de 20 entrées et 16 sorties . . . . . . . . . . . . 163

A.2.4 Registre 4 bits d'usage général et affichage 7 segments . . . . . 163

A.2.5 Registres 4 bits de sortie indiquant la position . . . . . . . . . . 163

A.2.6 Comportement après suicide de la cellule . . . . . . . . . . . . . 164

A.3 Jeu d'instructions et programmation du biodule $601 \ldots \ldots$

A.3.1 Diagramme syntaxique du langage asmBio2 . . . . . . . . . . 164

A.3.2 Variables ..................... 166

A.3.3 Bits de sortie . . . . . . . . . . . . . . 167

A.3.4 Bits d'entrée . . . . . . . . . . . . . . . . 167 
A.3.5 Jeu d'instructions . . . . . . . . . . . . . . . . . . . . 168

B Complete universal Turing machine microprogram

Bibliography

Curriculum vitae 


\title{
Chapter 1
}

\section{Introduction}

\begin{abstract}
"Since the apparition of visible life on Earth, three hundred and eighty million years had to pass so that a butterfly could learn to fly, another one hundred and eighty million years to make a rose without any other commitment than to be beautiful, and four geological eras such that human beings - unlike their great-grandfather pithecanthropus-, were able to sing better than the birds and to die of love. There is nothing honorable in the human intellect, in the golden age of science, having conceived of a way in which such an expensive and colossal multi-millenarian process, can return to the nothingness from where it came by the simple art of pressing a button."
\end{abstract}

\section{Gabriel García Márquez quoted from [70]}

Biological organisms are among the most intricate structures known to man, exhibiting highly complex behavior through the massively parallel cooperation of huge numbers of relatively simple elements, the cells. As the development of computing systems approaches levels of complexity such that their synthesis begins to push the limits of human intelligence, more and more engineers are beginning to look at Nature to find inspiration for the design of computing systems, both in software and in hardware [102]. The last three decades of investigations in the field of molecular biology (embryology, genetics, and immunology) has brought a clearer understanding of how living systems grow and develop. The principles used by Nature to build and maintain complex living systems are now available as a source of inspiration for the engineer [62]. 
In this introductory chapter, we present in Section 1.1 a short description of the motivations behind this work. In order to understand the basics of our approach, it is necessary to consider the biological inspiration of our project. Thus, in Section 1.2, we provide the required background by presenting an overview of the Embryonics project and its biological inspiration. Finally, in Section 1.3 we conclude the chapter with a brief outline of the overall structure of the thesis.

\subsection{Motivations}

The field of bio-inspired digital hardware was pioneered by John von Neumann [4]. A gifted mathematician and one of the leading figures in the development of the field of computer engineering, von Neumann dedicated the final years of his life on what he called the theory of automata [111]. His research, which was unfortunately interrupted by his untimely death in 1957, was inspired by the parallel between artificial automata, of which the paramount example are computers, and natural automata, such as the nervous system, evolving organisms, etc.

Through his theory of automata, von Neumann conceived a set of machines capable of many of the same feats as biological systems: evolution, learning, self-repair, selfreplication, etc. At the core of his approach was the development of self-replicating machines, that is, machines capable of producing identical copies of themselves. In developing his theory, von Neumann identified a set of criteria which had to be met in order to obtain useful biological-like behavior in computing machines [102]. These criteria rested on two fundamental assumptions:

$\Rightarrow$ Self-replication should be a special case of construction universality. That is, the self-replicating machines should be able not only to create copies of themselves, but also to construct any other machine, given its description. Such a feature would be a requirement to later obtain evolving systems.

$\Rightarrow$ The self-replicating machines should be universal computers, that is, capable of executing any finite (but arbitrarily large) program. Such machines were known to von Neumann: the requirement of logical universality is met by a class of automata known as universal Turing machines [30,42].

Von Neumann's research was, as we mentioned, never completed: the only machine he developed to any great extent was a theoretical model known as the universal constructor. Nevertheless, his theory of automata provides, even today, a firm foundation for the development of bio-inspired systems.

This thesis is part of a more general research project, called Embryonics [49, 52, $61,95,97]$ (a contraction of the words embryonic electronics), which aims at establishing a bridge between the world of biology and that of electronics, and in particular between 
biological organisms and digital circuits. One of the main goals of the Embryonics project is to determine if, given modern technology, Von Neumann's dream of a self-replicating machine can be realized in hardware.

Thus, inspired by von Neumann ideas, the main goal of this thesis is to realize, thanks to the Embryonics architecture, the first hardware implementation of a machine capable of self-replication and exhibiting universal computation, i.e., a universal Turing machine. The hardware architecture consists of a two-dimensional multicellular array of artificial cells (MICTREE cells) developed in our laboratory in the framework of the Embryonics project.

These MICTREE cells, a new kind of coarse-grained field-programmable gate array, are used for the implementation of multicellular artificial organisms with biologicallike properties, i.e., capable of self-repair and self-replication. But the design of the software programs defining the behavior of the MICTREE cells is difficult and had to be realized, until now, by hand. The mentioned approach often introduces errors in the software description. These errors are hard to debug, increasing the design time.

Thus, the second goal of this thesis is to develop a programming methodology to deal with complex applications in the Embryonics project. To do so, we present a detailed description of the implementation of some examples (from simple to complex), all based on a top-down design methodology characterized by the following processes:

$\Rightarrow$ the decomposition of the complete genome microprogram (the artificial genome) into a set of smaller sub-microprograms;

$\Rightarrow$ the systematic construction and optimization of these sub-microprograms thanks to the utilisation of binary decision trees and diagrams;

$\Rightarrow$ the rigorous organization of these sub-microprograms conforming to the Embryonics architecture (computation of the reset conditions and of the global configurations, of the $X$ and $Y$ coordinates, of the initial states and local configurations as a function of the $X$ and $Y$ coordinates, and finally computation of the genome's operational part describing, according to the coordinates, each gene of the artificial organism).

We finally developed a coherent family of software tools (MIC Editor, MIC Compiler, Visual BDD, MIC Sim, and a serial loader) that have been integrated into a single graphical design environment in order to achieve versatility and user-friendliness.

The software tools were designed taking into account the possibilities of evolution of the MICTREE cell architecture (for example more memory and larger internal registers, wider input/output busses). The resulting object oriented software architecture is easily scalable to meet all these constraint through minor changes to the code. The software tools provides the user with debugging capabilities not present in a real MICTREE network. 


\subsection{The Embryonics project}

In this section we want to provide an overview of the approach we adopted in the Embryonics project in order to adapt biological concepts and mechanisms to the world of electronics. After introducing the project and its main goals, we will present our approach to biological inspiration, and notably the model we created to try and unify bio-inspired systems into a common framework.

\subsubsection{Overview of the project}

Embryonics is a long-term research project, conceived not so much to achieve a specific goal, but rather to look for insights by applying new concepts to a known field. In our case, we try to obtain interesting results by applying biological concepts (i.e., concepts which are usually associated with biological processes) to computing, and notably to the design of reliable digital hardware.

The analogy between biology and electronics is not as farfetched as it might appear at a first glance. Aside from the more immediate parallel between the human brain and the computer, which has led to the development of fields such as artificial intelligence and is the inspiration for many applications (such as, for example, pattern recognition), a certain degree of similarity exists between the genome (the hereditary information of an organism) and a computer program.

The genome consists of a uni-dimensional string of data encoded in a base- 4 system. The DNA (DeoxyriboNucleic Acid), the macromolecule that encodes the genome, is a sequence written in a four-letter alphabet, the four bases: A (Adenine), C (Cytosine), G (Guanine), and T (Thymine). The information stored in the DNA is chemically decoded and interpreted to determine the function of a cell. A computer program is a unidimensional string of data encoded in a base-2 system (0 and 1). Stored in an electronic memory circuit, it is interpreted to determine the function of a processor.

Of course, carbon-based biology and silicon-based computing are different enough that no straightforward one-to-one relationship between the genome and a computer program (and indeed between any biological and computing process) can be established, except at a very superficial level. However, through careful interpretation, some basic biological concepts can be adapted to the design of computer systems, and some biological processes are indeed extremely interesting from a computer designer's point of view: for example, an organism's robustness (achieved by its healing processes) is unequaled in electronic circuits, while the natural process of evolution has produced organisms of a complexity that far exceeds that of modern computer systems.

It should be obvious that, biology being a very vast field, the biological concepts which could potentially be adapted to computer hardware are numerous, as are the approaches to applying such concepts to electronics. The first step in such an effort should therefore be to identify which aspects of the biological world will be studied, and define 
a unified framework which can meld them into a single project.

\subsubsection{The POE model}

Obviously, biological inspiration in computer science can assume a variety of different forms, and Embryonics does not attempt to cover all its possible aspects. Nevertheless, the scope of the project is such that it does involve many of the traditional bio-inspired systems, as well as the development of novel approaches.

In analogy to Nature, the POE model $[91,95]$ of bio-inspired hardware systems assumes the existence of three axes of organization of life: phylogeny, ontogeny, and epigenesis (Figure 1.1). The phylogenetic axis involves evolution, the ontogenetic axis involves the development of a single individual from its own genetic material, essentially without environmental interactions, and the epigenetic axis involves the learning through environmental interactions that takes place after formation of the individual $[54,94]$.

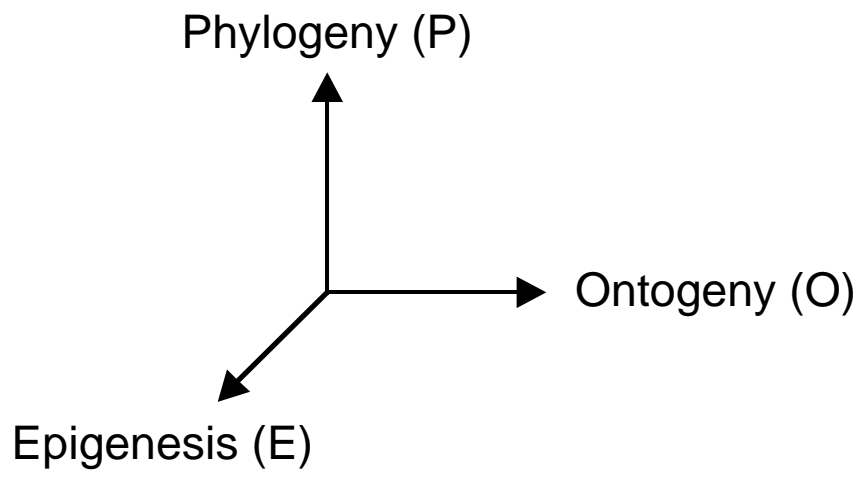

Figure 1.1: The POE Model.

\subsubsection{Phylogeny}

The first axis concerns the temporal evolution of the genetic program, the hallmark of which is the evolution of species, or phylogeny [17]. The multiplication of living organisms is based upon the reproduction of the genetic program (DNA), subject to an extremely low error rate at the individual level, so as to ensure that the identity of the offspring remains practically unchanged. Mutation (asexual reproduction) or mutation along with recombination (sexual reproduction) give rise to the emergence of new organisms. The phylogenetic mechanisms are fundamentally nondeterministic, with the mutation and recombination rate providing a major source of diversity. This diversity is indispensable for the survival of living species, for their continuous adaptation to a changing environment, and for the appearance of new species. 
Bio-inspired phylogenetic systems include the so-called evolvable systems: systems that, starting with a set of potential solutions to a problem, mimic the process of reproduction to generate potentially better solutions and imitate the process of natural selection to keep the better solutions and discard the bad ones. This process has served as the inspiration for engineering techniques like genetic algorithms [29,67], genetic programming [36,37], and evolutionary programming [21]. These ideas have been the inspiration for the development, in our laboratory, of an evolving hardware system (the Firefly machine) implementing online autonomous evolution [24].

Another, less conventional but very interesting example of a phylogenetic computer system is Tierra [83], an experimental project in which a "population" of programs is allowed to freely evolve inside a computer's memory. The very simple fitness criterion is survival in an environment with limited resources (in this case, the limited resource is the amount of memory available). Some very interesting and unexpected survival strategies emerged spontaneously, including parasitism (where tiny programs, which would not be able to survive by themselves, exploit the code belonging to other programs) and the evolution of more and more compact programs (smaller programs are more likely to survive in an environment where memory is the limited resource).

It appears, then, that the phylogenetic axis has already provided a considerable amount of inspiration to the development of computer systems. To date, however, its impact has been felt mostly in the development of software algorithms, and only marginally in the conception of digital hardware.

\subsubsection{Epigenesis}

The genome program is limited in the amount of information that it can store, implying that the complete specification of the organism in the DNA molecule is impossible. A well-known example is that of the human brain, consisting of approximately $10^{10}$ neurons and $10^{14}$ connections, far too large a number to be completely specified in a genome of approximately $3 \times 10^{9}$ characters in a 4-letter alphabet. Therefore, upon reaching a certain level of complexity, there must emerge a different process to allow the individual to integrate the vast quantity of interactions with the outside world. This process is known as epigenesis and includes primarily the nervous system, the immune system, and the endocrine system. These systems are characterized by a basic structure that is entirely defined by the genome (the innate part), which is then subjected to modification through lifelong interactions of the individual with the environment (the acquired part). The epigenetic processes can be loosely grouped under the heading of learning systems. Engineers have centered their attention on the nervous system, giving rise to the field of artificial neural networks (ANNs) [3,25].

ANNs cannot be expected to operate "correctly" (i.e. to produce the correct output) for all input patterns, a drawback which prevents their use in many applications. However, they have proved their worth (to the point that they are starting to be adopted in commercial systems) in applications such as voice and character recognition, where a 
limited margin of error is acceptable.

Our laboratory is also involved in research along the epigenetic axis, with the development of FAST (Flexible Adaptable-Size Topology) [76-78], a neural network with a dynamically-reconfigurable structure. Traditional ANNs have a fixed interconnection structure, and only the weights associated with the connections can be modified. By implementing the network using reconfigurable logic, FAST achieves on-line learning capabilities and can exploit an adaptable topology (two features which are essential to obtain true learning systems, as opposed to "learned" ones). While FAST is not the first neural network based on an adaptable topology [30, 67], it is unique in that it does not require intensive computation to reconfigure its structure, and can thus exist as a stand-alone machine that could be used, for example, in real-time control applications.

Epigenetic mechanisms have already had considerable impact on computer science, and particularly on software design, notably through the concept of learning. The parallel between a computer and a human brain dates to the very earliest days of the development of computing machines, and led to the development of the field known as artificial intelligence (AI) [113].

AI, which probably knew its greatest popularity in the seventies, tried to imitate the high-level processes of the human mind using heuristic approaches. After a number of years in the spotlight, this field seems today to have lost momentum, but not without leading to useful applications (such as expert systems) and major contributions to the development of novel algorithms (for example, IBM's well-known Deep Blue computer exploits algorithms largely derived from AI research).

\subsubsection{Ontogeny}

The phylogenetic and epigenetic axes of the POE model cover the great majority of existing bio-inspired systems. The development of a multi-cellular biological organism, however, involves a set of processes which do not belong to either of these two axes. These processes correspond to the growth of the organism, i.e. to the development of an organism from a mother cell (the zygote) to the adult phase. The zygote divides, each offspring containing a copy of the genome (cellular division). This process continues (each new cell divides, creating new off-spring, and so on), and each newly formed cell acquires a functionality (i.e., liver cell, epidermal cell, etc.) depending on its surroundings, i.e., its position in relation to its neighbors (cellular differentiation).

Cellular division is therefore a key mechanism in the growth of multi-cellular organisms, impressive examples of massively parallel systems: the $6 \times 10^{13}$ cells of a human body, each a relatively simple elements, work in parallel to accomplish extremely complex tasks (the most outstanding being, of course, intelligence). If we consider the difficulty of programming parallel computers (a difficulty which has led to a decline in the popularity of such systems), biological inspiration could provide some relevant insights on how to handle massive parallelism in silicon. 
A fundamental feature of biological organisms is that each cell contains the blueprint for the entire organism (the genome), and thus can potentially assume the functionality of any other cell: no single cell is indispensable to the organism as a whole. In fact, cells are ceaselessly being created and destroyed in an organism, a mechanism at the base of one of the most interesting properties of multi-cellular organisms: healing.

The mechanisms of ontogeny (cellular division and cellular differentiation), unlike those of epigenesis and phylogeny, are completely deterministic, and are thus, in theory, more easily adaptable to the world of digital circuits (which is by nature deterministic). In spite of this, the ontogenetic axis has been almost completely ignored by computer scientists, despite a promising start in the fifties with the work of John von Neumann, who developed a theoretical model of a universal constructor, a machine capable of constructing any other machine, given its description [111]. Given a description of itself, the universal constructor can then self-replicate, a process somewhat analogous to cellular division. These ideas have been the inspiration for the development of embryonic hardware with self-repair and self-replication capabilities [51,60,102].

We will describe von Neumann's machine, one of the fundamental sources of inspiration for the Embryonics project in general and for this work in particular, in Chapter 2.

Probably the main obstacle to the development of self-replicating machines was the impossibility of physically creating self-replicating hardware (we should mention that, unfortunately, electronic circuits in the 1950s were too primitive to allow von Neumann's machine to be realized). In fact, such machines require a means to transform information (i.e. the description of a machine) into hardware, and such a means was definitely unpractical until recently. The introduction of programmable logic circuits (FPGAs) [12,108], by demonstrating the feasibility of such a process, was an important step towards the development of self-replicating machines.

\subsection{Outline of the thesis}

This thesis is organized in seven chapters, the first Chapter being this introduction. Chapter 2 is dedicated to von Neumann's research on the subject of his universal constructor, a self-replicating cellular automaton [111], and to its further refinements at the hands of von Neumann's successors, which made it the basis for all further research on self-replication.

Chapter 3 presents the MICTREE (for tree of micro-instructions) cell, a new kind of coarse-grained field-programmable gate array (FPGA) developed in the framework of the Embryonics project. This cell will be used for the implementation of multicellular artificial organisms with biological-like properties, i.e., capable of self-repair and selfreplication. Two applications of limited complexity (a random number generator and a 2-by-n hybrid cellular automaton) illustrate the top-down programming methodology.)

Chapter 4 contains a brief introduction to Turing machines (Section 4.1) and the details of the implementation of a self-replicating and self-repairing specialized Turing 
machine, a parenthesis checker, based on a multicellular array of cells (Section 4.2 to Section 4.4). Our systematic programming methodology is again emphasized.

Chapter 5 presents, in Section 5.1, a brief overview of the universal Turing machine. In Section 5.2 we introduce the basics of the Embryonics architecture based on a multicellular array of cells. We then present the architecture of an ideal and of an actual universal Turing machine able to self-replicate. In Section 5.3 we describe the PICOPASCAL language and a hardware implementation of a PICOPASCAL interpreter, which is necessary for the understanding of our Embryonics implementation. Section 5.4 describes the detailed implementation of a self-replicating and self-repairing universal Turing machine with a particular emphasis on the programming methodology. Finally, a discussion of our results is presented in the last section of the chapter (Section 5.5).

Chapter 6 presents a description of the different software tools that have been developed to deal with the programming of complex Embryonics applications. We show how all these software tools have been integrated into a single graphical design environment in order to achieve versatility and user-friendliness. This chapter describes particularly in detail the MIC Sim software (MICTREE network simulator) and the use of the Visual MICTREE design environment.

The last chapter (Chapter 7) presents some conclusions and discusses the goals achieved with the present work. It concludes with a few considerations on possible future developments for the Embryonics project in general and this work in particular.

The body of the thesis will be followed by two annexes: the first (Appendix A) will describe the detailed specifications of the MICTREE artificial cell (registers, input/output busses, routing system, memory, and programming language); the second (Appendix B) is the listing of the complete genome microprogram describing our universal Turing machine implementation. 



\title{
Chapter 2
}

\section{History of Self-Replicating Machines}

\author{
"Anybody who looks at living organisms knows perfectly well that \\ they can produce other organisms like themselves. This is their \\ normal function, they wouldn't exist if they didn't do this, and \\ it's plausible that this is the reason why they abound in the world. \\ In other words, living organisms are very complicated aggregations \\ of elementary parts, and by any reasonable theory of probability \\ or thermodynamics highly improbable. That they should occur in \\ the world at all is a miracle of the first magnitude..."
}

\author{
John von Neumann \\ quoted from [41]
}

The notion of self-replication was inspired by Nature and first studied by John von Neumann in the late 1940s. He was interested in the question of whether a machine can self-replicate, and introduced the fundamental notion of universal constructor.

The existing approaches to the self-replication of computing systems are essentially derived from the work of John von Neumann [4]. Unfortunately, the state of the art in the fifties restricted von Neumann's investigations to a purely theoretical level, and the work of his successors mirrored this constraint.

In Section 2.1, we present von Neumann's research on the subject of his universal constructor, a self-replicating cellular automaton [111], and discuss how it was further refined by von Neumann's successors and became the basis for all further research on self-replication. Section 2.2 will describe the new approach to self-replicating machines, i.e., the development of self-replicating loops as suggested by Langton in the 80s [38]. Sections 2.3 and 2.4 will briefly describe the design of self-replicating loops with computing, and universal computing capabilities, respectively, while Section 2.5 concludes this chapter by introducing our original Embryonics approach. 


\subsection{Self-replicating automata}

The early history of the theory of self-replicating machines is basically the history of John von Neumann's research on the subject $[26,92,111]$. Stanislaw Ulam and John von Neumann introduced in the late 1940's the cellular automaton (CA) model [16, 106, 111, 119] to provide a formal framework for investigating self-replication. CA are dynamic systems in which space and time are discrete.

CA are arrays of elements, or cells, all identical in structure and behavior. At regular, discrete intervals (iterations), the state of all elements is updated synchronously, depending on the current state of the element itself and on that of its neighbors, according to a set of transition rules [102]. The identical set of rules contained in each cell forms essentially a finite state machine, usually specified in the form of a rule table, with an entry for every possible configuration of states for the neighborhood [92].

To summarize, a cellular automaton is defined by the following parameters:

$\Rightarrow$ A number of dimensions, usually one or two, rarely three, and almost never four or more. All the automata used to model self-replication are two-dimensional.

$\Rightarrow$ A set of states and an initial configuration, defining the state of all the elements of the array at iteration 0. While there is no theoretical limit to the number of states in an automaton, for practical considerations very few automata use more than a handful.

$\Rightarrow$ A neighborhood, which specifies which neighbors will have an effect on an element's next state. By far the most common for two-dimensional automata are the neighborhood of 5 (the element itself plus its cardinal neighbors to the north, south, east, and west), also known as the von Neumann neighborhood and neighborhood of 9 (the element itself plus its neighbors to the north, south, east, west, northeast, southeast, southwest, and northwest), also known as the Moore neighborhood.

$\Rightarrow$ A set of transition rules, used to compute an element's next state depending on the neighborhood. The rules can be expressed either as an algorithm or exhaustively as a lookup table. In the latter case, the total number of rules necessary to exhaustively define a cellular automaton is $S^{n}$, where $S$ is the number of states and $n$ the neighborhood. In practice, the number of required rules can, in many cases, be considerably reduced, but the lookup table for a complex automaton (i.e., one with many states) can nevertheless reach a very important size.

As should now be obvious, cellular automata are not a model which can easily be applied to digital hardware. The need for each element to access the transition rules, coupled with the large number of elements required for complex behavior, is a serious drawback for an electronic implementation. In addition, while cellular automata can be a powerful model for certain kinds of applications where the transition rules are relatively 
simple and known in advance (e.g., the modelization of the behavior of gases), the design of a complex automaton is extremely difficult: the absence of global rules imply that complex automata (i.e., configurations which span a large number of elements) have to be handled exclusively at the local level, and no formal approach is available to aid in this task [102].

\subsubsection{Von Neumann's self-replicating machines}

Von Neumann, confronted with the lack of reliability of computing systems, turned to Nature to find inspiration in the design of fault-tolerant computing machines. Natural systems are among the most reliable complex systems known to man, and their reliability is a consequence not of any particular robustness of the individual cells (or organisms), but rather of their extreme redundancy: redundancy at the cellular level (each single cell contains a complete copy of the blueprint of the whole organism, i.e., the genome), at the organismic level (cells are able to self-replicate), and at the population level (organisms are able to self-reproduce).

Von Neumann finally chose self-replication (the capability of producing an exact copy of an original) as the basic process of his research and attempted to design selfreplicating computing machines, which he called artificial automata, as opposed to natural automata, that is, biological organisms.

In order to find an approach to self-replication more amenable to a rigorous mathematical treatment, von Neumann, following the suggestion of his colleague mathematician Stanislaw Ulam, developed a cellular model [111] to study this problem. Von Neumann used a two-dimensional CA with 29 states per cell and a five-cell neighborhood. He showed that a universal computer can be embedded in a such cellular space, namely, a device whose computational power is equivalent to that of a universal Turing Machine [30]. Even if it was never completed, this model, further refined by von Neumann's successors, was the basis for all further research on self-replication.

\subsubsection{Von Neumann's cellular automaton}

In von Neumann's work, self-replication is always presented as a special case of universal construction, that is, the capability of building any machine given its description (Figure 2.1). This approach was maintained in the design of his cellular automaton, which is therefore much more than a self-replicating machine. The complexity of its purpose is reflected in the complexity of its structure, based on three separate components:

$\Rightarrow$ A memory tape, containing the description of the machine to be built, in the form of a one-dimensional string of elements. In the special case of self-replication, the memory contains a description of the universal constructor itself ${ }^{1}$ (Figure 2.2).

\footnotetext{
${ }^{1}$ The memory of von Neumann's automaton bears a strong resemblance to the biological genome. This
} 


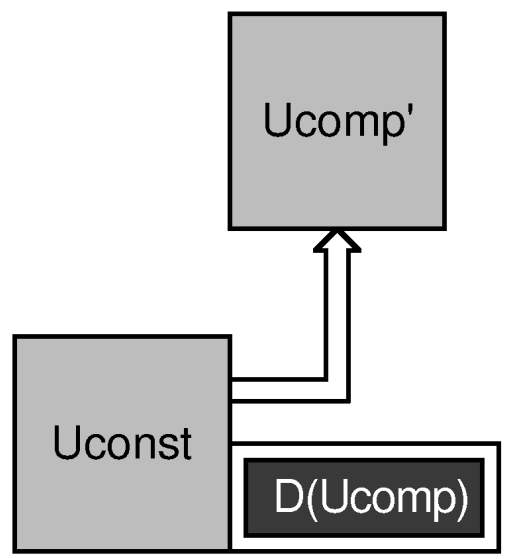

Figure 2.1: Universal construction of von Neumann's automaton: a possible configuration can implement a universal constructor Uconst. Then, given the description D(Ucomp) of any one machine Ucomp, including a universal Turing machine, the universal constructor can build a specimen of this machine (Ucomp') in the cellular space.

$\Rightarrow$ The constructor itself, a very complex machine capable of reading the memory tape and interpreting its contents.

$\Rightarrow$ A constructing arm, directed by the constructor, used to build the offspring (i.e., the machine described in the memory tape). The arm moves across the space and sets the state of the elements of the offspring to the appropriate value.

The implementation as a cellular automaton is no less complex. Each element has 29 possible states, and thus, since the next state of an element depends on its current state and that of its four cardinal neighbors, $29^{5}=20,511,149$ transition rules are required to exhaustively define its behavior. If we consider that the size of von Neumann's constructor is of the order of 100,000 cells, we can easily understand why a hardware realization of such a machine is not really feasible.

In fact, as part of the Embryonics project $[49,52,61,95]$, a hardware implementation of a limited number of von Neumann's cells was realized [9,93]. These cells were implemented using the technology of field-programmable gate arrays (FPGAs) [110]. By carefully designing the hardware structure of each element, the amount of memory required to host the transition rules was considerably reduced. Nevertheless, the system remains a demonstration tool, as it consists of a few elements only, barely enough to illustrate the behavior of a tiny subset of the entire machine.

Before we continue, we should mention that von Neumann went one step further in the design of his universal constructor. If we consider the universal constructor from a resemblance is even more remarkable when considering that the structure of the genome was not discovered until after the death of von Neumann. 


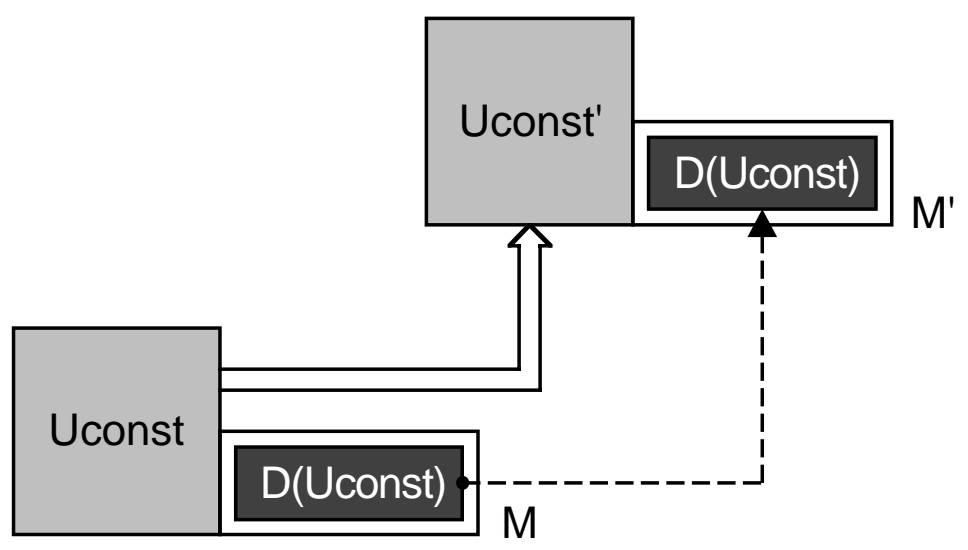

Figure 2.2: Self-replication of the universal constructor: given the description $D$ (Uconst) of the constructor itself, it is then possible to build a copy of the constructor in the cellular space: the constructor interprets first the description D(Uconst) to build a copy Uconst' whose memory $M$ ' is empty (translation process), and then copies the description $D$ (Uconst) from the original memory $M$ to the new memory $M$ ' (transcription process).

biological viewpoint, we can associate the memory tape with the genome, and thus the entire constructor with the ribosome (which would imply a parallel between the automaton's elements and molecules).

However, the constructor, as we have described it so far, has no functionality outside of self-replication. Von Neumann recognized that a self-replicating machine would require some sort of functionality to be interesting from an engineering point of view, and postulated the presence of a universal computer (in practice, a universal Turing machine, an automaton capable of performing any computation) alongside the universal constructor (Figure 2.3).

Von Neumann's constructor and universal computer can thus be regarded as a unicellular organism (a mother cell), containing a genome stored in the form of a memory tape, read and interpreted by the universal constructor (the ribosome) to direct the construction of a complete copy of itself (the daughter cell). Von Neumann's self-replication is the artificial equivalent of natural cells' self-replication, i.e., cellular division.

\subsubsection{Von Neumann's successors}

The extreme complexity of von Neumann's universal constructor has so far prevented any kind of physical implementation (apart from the small demonstration unit we mentioned). But further, even the simulation of a cellular automaton of such complexity was far beyond the capability of early computer systems. Today, such a simulation is starting to 


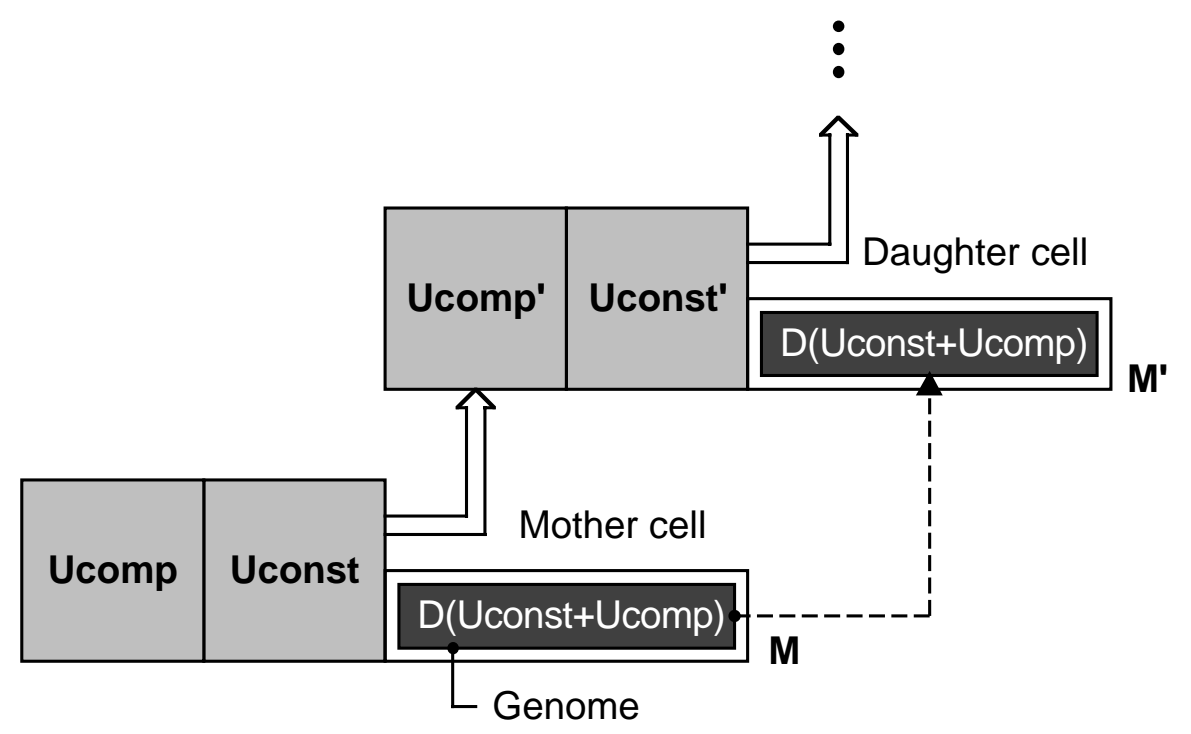

Figure 2.3: Self-replication of a universal computer: by attaching to the constructor a universal computer Ucomp (a universal Turing machine), and by placing the description $D(U c o n s t+U c o m p)$ in the original memory $M$, the universal constructor produces a copy of itself (Uconst') and a copy of the universal computer (Ucomp') through the mechanism described in Figure 2.2 (interpretation and then duplication of the description D).

be conceivable. Umberto Pesavento, thanks to a modified cell with 32 states, developed a simulation of a simplified version of von Neumann's universal constructor [80]. The computing power available did not allow him to simulate either the entire self-replication process (the length of the memory tape needed to describe the automaton would have required too large an array) or the Turing machine necessary to implement the universal computer, but he was able to demonstrate the full functionality of the constructor. Considering the rapid advances in computing power of modern computer systems, we can assume that a complete simulation could be envisaged within a few years [102].

The impossibility of achieving a physical realization did not however deter some researchers from trying to continue and improve von Neumann's work [5,39,71]. Arthur Burks, for example, in addition to editing von Neumann's work on self-replication [14, 111], also made several corrections and advances in the implementation of the cellular model. Codd [16], by altering the states and the transition rules, managed to simplify the constructor by a considerable degree. He reduced the number of states required for a self-replicating universal constructor computer from 29 to 8 [35, 92]. However, without in any way lessening these contributions, we can say that no major theoretical advance in the research on self-replicating automata occurred until C. Langton [38], in 1984, opened a second stage in this field of research. 


\subsection{Self-replicating loops}

Von Neumann's universal constructor was very complex because it tried to implement self-replication as a particular case of construction universality, i.e. the capability of constructing any other automaton, given its description. C. Langton approached the problem somewhat differently [38], by attempting to define the simplest cellular automaton capable exclusively of self-replication [102]. He dropped the condition that the self-replicating unit must be capable of universal construction and computation. Langton's mechanism is based on an extremely simple configuration in Codd's automaton [16] called the periodic emitter, itself derived from the periodic pulser organ in von Neumann's automaton [111]. The cell of Langton's automaton is a finite state machine with only 8 states. The future state, as with von Neumann's automaton, depends on the present state of the cell itself and its four cardinal neighbors. The exhaustive definition of the future state, the transition table, contains only 219 lines, a very small subset of the theoretically required $8^{5}=262,144$ lines (thanks to the use of default rules and symmetry assumptions).

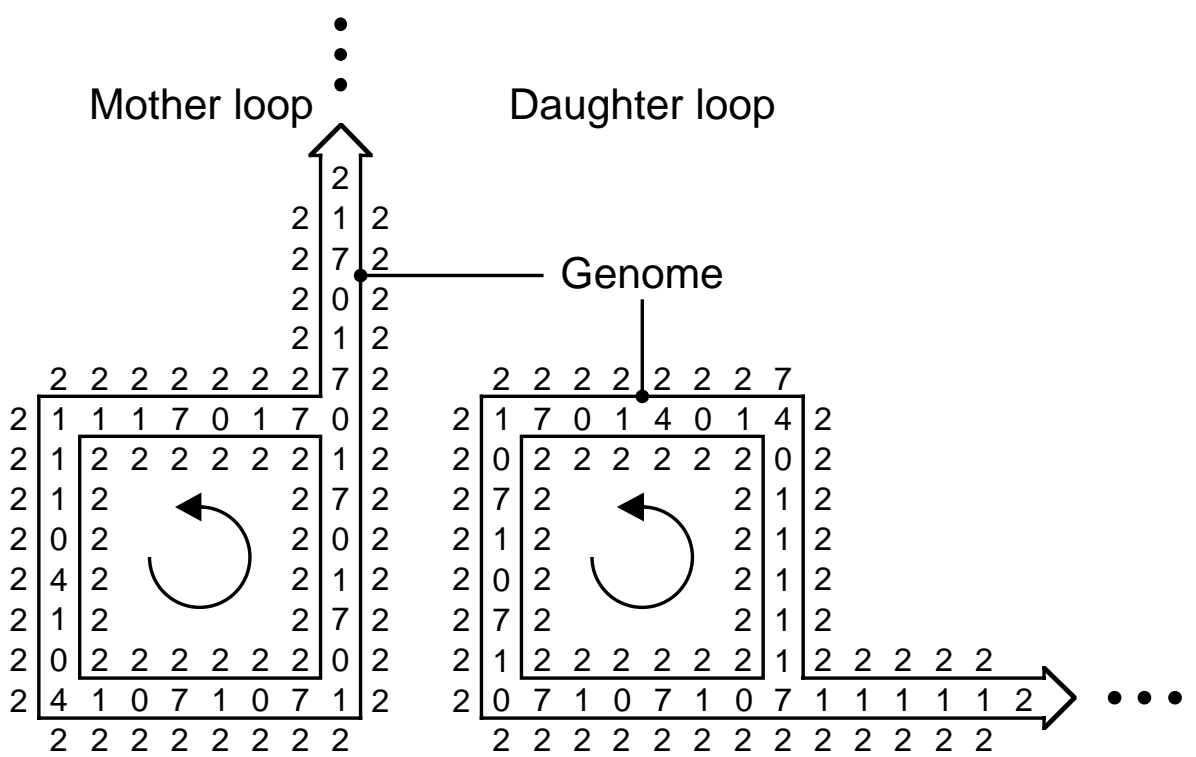

Figure 2.4: In Langton's self-replicating loop, the genome, which turns counterclockwise, is characterized by the sequence, read clockwise: 170170 1701701701701401401111 . The signals "1" are ignored, the signals "70" cause the extension of the constructing arm by one cell, while the signals " 40 ", repeated twice, cause the arm to turn $90^{\circ}$ counterclockwise. After 151 clock periods, the left loop (the mother loop) produces a daughter loop, thus obtaining the self-replication of Langton's loop. The genome is both interpreted (construction of a copy at the end of the constructing arm: translation process) and copied (duplication at the junction of arm and loop: transcription process). 
Langton proposed a configuration in the form of a loop (Figure 2.4), with a constructing arm (pointing to the north in the left loop and to the east in the right loop) and a replication program, or genome, which turns counterclockwise and is surrounded by sheath cells in state 2 (this latter state is represented by the number 2 in Figure 2.4). After 151 clock periods, the left loop (the mother loop) produces a daughter loop, thus achieving self-replication.

Referring again to biological definitions, we observe that Langton's self-replicating loop is a unicellular organism; its genome, defined in Figure 2.4, comprises 28 elements (which would be "molecules" in biology and are "cells" in CA vocabulary) and is a subset of the complete loop, which requires 94 elements.

In summary:

$\Rightarrow$ the size of Langton's loop is perfectly reasonable, since it requires 94 elements, thus allowing complete simulation;

$\Rightarrow$ there is neither universal construction nor universal calculation: the loop does nothing but replicate itself; comparing Figure 2.2 and Figure 2.4 reveals that Langton's self-replicating loop represents a special case of von Neumann's self-replication; the loop is a non-universal constructor, capable of building, on the basis of its genome, a single type of machine: itself.

Further simplifications to Langton's automaton were introduced by Byl [15], who reduced the internal sheath and the number of states per cell, the number of transition rules, and the number of non-quiescent cells in the initial configuration. His self-replicating loop comprised 12 elements and was embedded in a six-state cellular space. Reggia et al. [84] managed to remove also the external sheath, thus designing the smallest self-replicating loop known to date. This self-replicating loop comprised 6 elements and was embedded in a six-state cellular space.

Morita and Imai [68,69] implemented simple self-replication loops in reversible cellular automata, which is a special type of CA in which every configuration of the cellular space has at most one predecessor. A prime motivation for studying such systems stems from the observation that computers based on reversible logic can be more efficient (e.g., faster, smaller, with larger memories, and lower energy consumption) than classical ones $[7,8,92]$.

Stauffer and Sipper [99] studied the self-replication issue using the L-system model $[43,81]$. L-systems are naturally well-suited for modeling growth processes, of which replication is a special case. They (1) showed that L-systems can be used to specify self-replication structures and (2) explored the relationship between L-systems and CAs. They worked in a two-dimensional cellular space with a von Neumann neighborhood and concluded that the bridge between L-systems and CAs seems to offer a promising approach in the study of self-replication, and more generally, of growth processes in CAs [92]. 


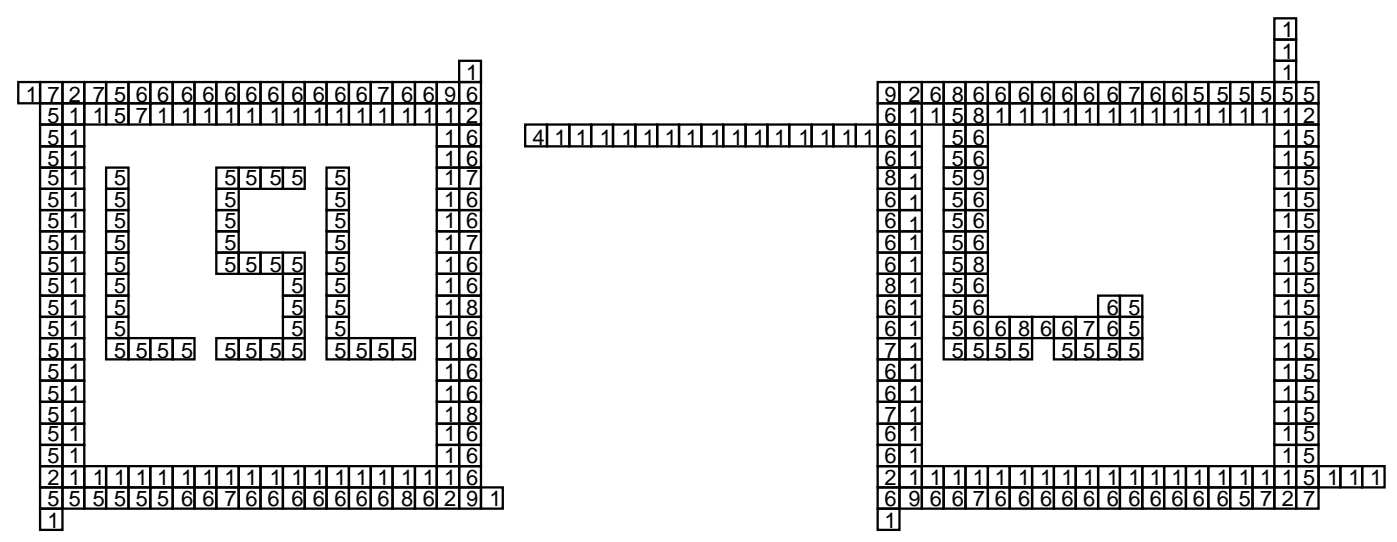

Figure 2.5: Tempesti's self-replication automaton. Tempesti's self-replicating loop has an attached executable program that is duplicated and executed in each of its copies. The cellular space is two-dimensional, nine-neighbor, with ten states per cell.

In a more recent paper, Stauffer and Sipper [100] observed that self-replicating loops presented to date are essentially worlds unto themselves, inaccessible to the observer once the replication process is launched. Thus, they presented a new self-replicating loop which allows for user interaction. In this approach, the user can start the loop's replication and induce its destruction. In this work they presented a physical implementation embedded in a two-dimensional electronic wall, the BioWall $[58,98]$.

Given their modest complexity, at least relative to von Neumann's automaton, all of the mentioned automata have been thoroughly simulated.

\subsection{Self-replicating loops with computing capabilities}

The loops of the previous section exhibit only rudimentary computing and constructing capabilities, their sole functionality being that of self-replication. Lately, new attempts have been made to redesign Langton's loop in order to embed calculation capabilities. Tempesti's loop [101] is a self-replicating automaton which preserves some of the more interesting features of Langton's loop (in particular, it preserves the structure based on a square loop to dynamically store information, and the concept of a constructing arm); nevertheless, Tempesti introduced important modifications to Langton's design. Tempesti's loop attaches an executable program that is duplicated and executed in each of its copies. The programs is stored within the loop, interlaced with the replication code (Figure 2.5). This process is demonstrated for a simple program that writes out (after the loop's replication) LSL, acronym of the Logic Systems Laboratory [102]. 


\subsection{Self-replicating loops with universal computing capabilities}

Perrier et al.'s [79] went beyond Tempesti's demostration of finite computation, constructing a self-replicating loop with universal computational capabilities (Figure 2.6). The system consists of three parts, loop, program, and data, all of which are replicated before the program's execution on the given data. In Figure 2.6, $P$ and $D$ denote states belonging to the set of program states and to the set of data states, respectively. The loop was simulated in its entirety, thus demonstrating a viable, self-replicating machine with programmable capabilities.

The universal computational model chosen for this work was the W-machine, introduced by Hao Wang [112] and named for him by Lee [40], who explored its relation with finite automata. A W-machine is like a Turing machine, save that its operation at each time step is guided not by a state table but by an instruction from the following list [2]:

$\Rightarrow$ PRINT 0

$\Rightarrow$ PRINT 1

$\Rightarrow$ MOVE DOWN

$\Rightarrow$ MOVE UP

$\Rightarrow$ IF 1 THEN $(n)$ ELSE (next)

$\Rightarrow$ STOP

The complete program for a Turing machine is a finite ordered list of instructions (a program) equivalent to the state table. After execution of an instruction of the first four types, control is automatically transferred to the next instruction. The conditional jump IF ... transfers control to the $n$-th instruction if the square under scan is a 1 symbol, otherwise it transfers control to the next instruction.

Adding functionality to Langton's loop is, in fact, not possible without major alterations. Perrier et al. developed a relatively complex automaton, in which a two-tape Turing machine was appended to Langton's loop. This automaton exploits Langton's loop as a sort of carrier: the first function of Perrier's loop is to allow Langton's loop to build a copy of itself. The main function of the offspring is to determine the location of the copy of the Turing machine. Once the daughter loop is ready, a messenger runs back to the mother loop and starts to duplicate the Turing machine, a process completely disjoint from the operation of the loop. When the copy is finished, the same messenger activates the Turing machine in the mother loop (the machine had to be inert during the replication process in order to obtain a perfect copy). The process is then repeated in each offspring until the space is filled [79]. 


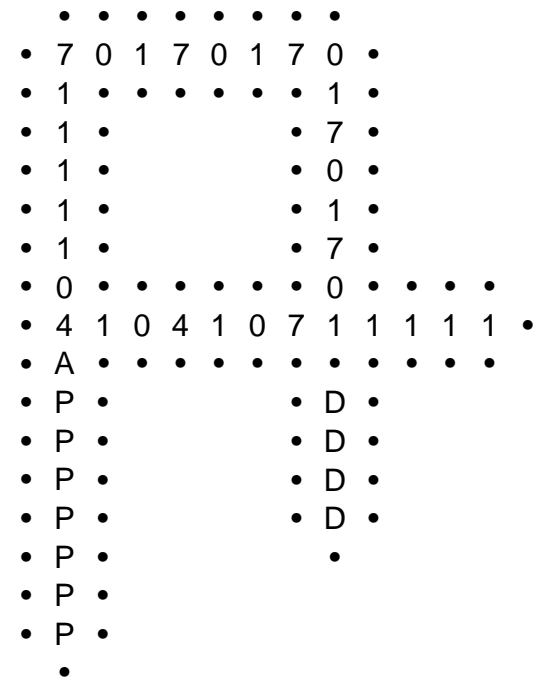

Figure 2.6: Perrier et al.'s self-replicaion loop. The system consists of three parts, loop, program, and data, all of which are replicated before the program's execution on the given data. The cellular space is twodimensional, with a neighborhood of 5 and 63 states per cell. P denotes a state belonging to the set of program states. $D$ denotes a state belonging to the set of data states. A is a state which indicates the position in the program.

The automaton thus becomes a self-replicating universal Turing machine, a powerful construct which is unfortunately handicapped by its complexity: in order to implement a Turing machine, the automaton requires a very considerable number of additional states (63), as well as a large number of additional transition rules. This complexity, while still relatively minor compared to von Neumann's universal constructor, is nevertheless too high to be considered for a hardware application. So once again, adapting Langton's loop to fit our requirements proved too complex to be efficient [102].

\subsection{Self-replication of Turing machines on a multicellular array: The Embryonics approach}

Arbib [2] was the first to suggest a truly "cellular" automaton, in which every cell contains a complete copy of the genome, and a hierarchical organization, where each cell is itself decomposed into smaller, regular parts, the "molecules". Unlike all previous realizations, this new architecture is a truly "multicellular" artificial organism.

This key idea was the basis of the Embryonics (embryonic electronics) project, under development by Mange and his colleagues at Lausanne, by Marchal and his colleagues at 
Neuchâtel, and by Tyrrell and his colleagues at York. The ultimate objective of this project is the construction of large-scale integrated circuits exhibiting properties such as growth, self-repair (healing), and self-replication, found, up until now, only in living beings [52, $56,60-62,73-75,104]$.

With a more theoretical goal in mind, Mange et al. have shown, in order to compare the capabilities of the Embryonics project with those of von Neumann's self-replicating automaton [111], that an artificial multicellular organism can implement a specialized Turing machine and exhibit the properties of self-replication and self-repair [50]. The example that was settled upon is that of a parenthesis checker, as described by Minsky [64]. The function of the machine is to decide whether a sequence of left (open) and right (closed) parentheses is well-formed (i.e., if for every open parenthesis in the sequence there exists a corresponding closed parenthesis). A specialized Turing machine for checking parentheses consists of a tape, decomposed into squares, and a finite-state machine with a read/write head. The detailed implementation of this machine is described in Chapter 4.

Encouraged by the interesting results obtained with the multicellular implementation of the specialized Turing machine, Restrepo and Mange $[87,88,90]$ presented a first implementation of a multicellular universal Turing machine (UTM) with self-replication and self-repair capabilities. Chapter 5, which constitutes the core of this thesis, will describe the detailed implementation of that UTM, which was made possible thanks to the Embryonics architecture. 


\section{Chapter 3}

\section{The MICTREE Cell}

Living organisms are complex systems exhibiting a range of desirable characteristics, such as evolution, adaptation, and fault tolerance, that have proved difficult to realize using traditional engineering methodologies. The last three decades of investigations in the field of molecular biology (embryology, genetics, and immunology) has brought a clearer understanding of how living systems grow and develop. The principles used by Nature to build and maintain complex living systems are now available for the engineer to draw inspiration from [62].

The growth and the operation of all living beings are directed through the interpretation, in each of their cells, of a chemical program, the DNA. This program, called genome, is the blueprint of the organism and consists of a complex sequence written with an alphabet of four characters: A, C, G, and T. This process is the source of inspiration for the Embryonics (embryonic electronics) project [49, 52, 61, 95], whose final objective is the conception of very large scale integrated circuits endowed with properties usually associated with the living world: self-repair and self-replication.

In this chapter, we will present the MICTREE (for tree of micro-instructions) cell, a new kind of coarse-grained field-programmable gate array (FPGA) developed in the framework of the Embryonics project. This cell will be used for the implementation of multicellular artificial organisms with biological-like properties, i.e., capable of selfrepair and self-replication.

Sections 3.1 and 3.2 present a general description and the main features of the MICTREE cell respectively. Through the examples in Sections 3.3 and 3.4 we show that our artificial organisms possess the above mentioned biological-like properties of selfreplication and self-repair. Section 3.5 presents a short introduction to the software tools developed to work with our artificial cell (a detailed description can be found in Chapter 6). Finally, Section 3.6 realize a short analysis of the reliability of the MICTREE cell architecture. 


\subsection{General description}

The MICTREE cell is a new kind of coarse-grained field-programmable gate array (FPGA), which will be used for the implementation of multicellular artificial organisms with biological-like properties $[54,85,96]$. The environment in which our quasi-biological artificial cells will develop consists of a finite (but as large as desired) two-dimensional space of silicon. This space is divided into rows and columns whose intersections define the cells. Since such cells (small processors and their memory) have an identical physical structure, i.e., an identical set of logic operators and of connections, the cellular array is homogeneous. Only the state of the cell, that is, the content of its registers, can differentiate it from its neighbors.

In all living beings, the string of characters which makes up the DNA, the genome, is executed sequentially by a chemical processor, the ribosome. Drawing inspiration from this mechanism, MICTREE is based on a binary decision machine (BDM) [47] (our ribosome), which sequentially executes a microprogram (our genome). In addition, the artificial cell is composed of a random access memory (RAM), and a communication system implemented by a switch block (SB) (Figure 3.1).

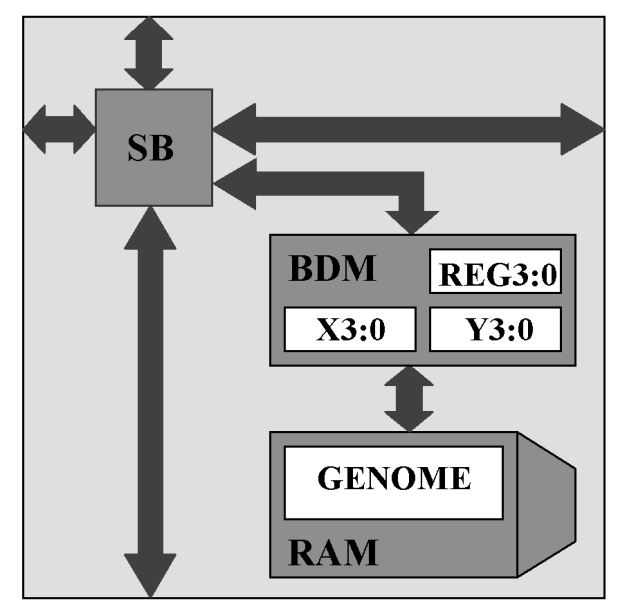

Figure 3.1: MICTREE block diagram; SB: switch block; BDM: binary decision machine; RAM: random access memory; REG3:0: state register; X3:0: horizontal coordinate; Y3:0: vertical coordinate.

The binary decision machine executes a microprogram of up to 1024 instructions, which is stored in the RAM. The microprogram itself is decomposed in sub-programs that are equivalent to the different parts of the genome: the genes. The execution of a specific gene depends on the physical position of the cell in the two-dimensional array, i.e., on its coordinates.

MICTREE's instructions are essentially test and assignment operators (for the calculation of the genes, of the coordinates, and of the genome) and configuration directives 
(to program the busses connecting each cell to its immediate neighbors). The MICTREE cell sequentially executes microprograms written using the following set of instructions:

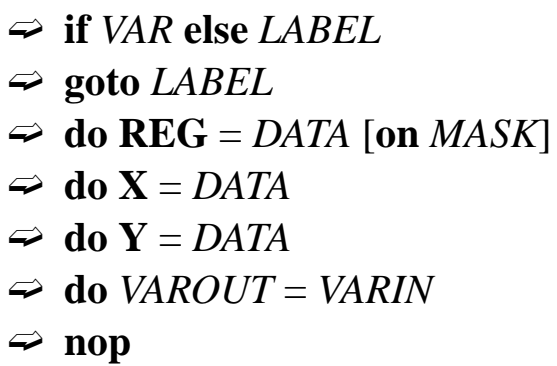

These instructions define the new MIC programming language that we will use to program the MICTREE artificial cell.

The state register $R E G$ and both coordinate registers are 4 bits wide (REG3:0, X3:0, and Y3:0). The 4 bits of MASK allow us to select which of the bits of REG3:0 will be affected by the assignment. By default, $M A S K=1111$ (all bits are affected).

The variables VAROUT correspond to the four cardinal output busses, for a total of 16 bits (Figure 3.13a):

$$
\text { VAROUT } \in\{\text { SO3:0, WO3:0,NO3:0,EO3:0 }\}
$$

while the variables VARIN correspond to the four cardinal input busses and the register REG, for a total of 20 bits:

$$
V A R I N \in\{S I 3: 0, \text { WI3:0, NI3:0, EI3:0, REG3:0 }\}
$$

remembering that VAROUT and VARIN can never refer to the same cardinal direction (Figure 3.13b).

The test variables VAR include the set VARIN and the following additional variables:

$$
V A R \in\{\text { VARIN, WX3:0,SY3:0,G }\}
$$

where $W X$ is the coordinate of the nearest west neighbor, $S Y$ is the coordinate of the nearest south neighbor, and $G$ is a global variable, usually reserved for the synchronization clock.

The coordinates are transmitted from cell to cell serially, but are computed in parallel. Therefore, each cell performs a series-to-parallel conversion on the incoming coordinates $W X$ and $S Y$ (Figure 3.2) of the western and southern neighbors respectively, and a parallelto-series conversion of the coordinates $X$ and $Y$ it computes and propagates to the east and north. By default (that is, with the external connections $W X$ and $S Y$ not connected), the mother cell recognizes the values $W X=S Y=0$. 
The genome microprogram is also coded serially. It enters through the DIN pin (Figure 3.2) of the mother cell and is then propagated through its DOUT pin, according to the cellular division path determined by the user.

The pins $C K$ and $C L R$ ' are used for the propagation of the clock signal and to reset the binary decision machine respectively, while the signal BYP (bypass), connecting all the cells of a column, is used for self-repair.

The size of the artificial organism embedded in an array of MICTREE cells is limited in the first place by the coordinate space $(X=0 \ldots 15, Y=0 \ldots 15)$, that is, a maximum of 256 cells in our current implementation), and then by the size of the memory of the binary decision machine storing the genome microprogram (1024 instructions).

The MICTREE cell has been embedded into a plastic demonstration module, the BIODULE 601 (Figure 3.2). Each module can be easily joined with others, like a LEGO, to build artificial organisms as large as desired.

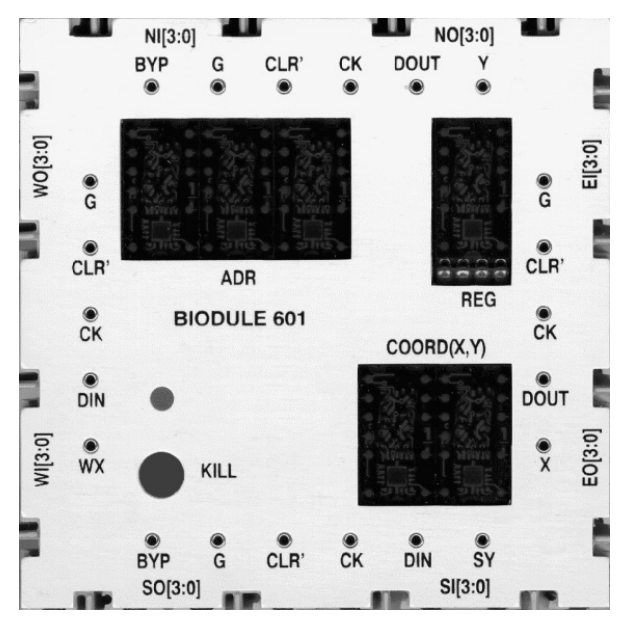

Figure 3.2: BIODULE 601 demonstration module.

\subsection{MICTREE features}

MICTREE is a truly cellular automaton and its conception derives from the study of the multicellular living beings. It relies on three fundamental features: multicellular organization (the artificial organism is decomposed into a finite number of cells, where each cell realizes a unique function, described by a sub-program called the gene of the cell), cellular differentiation (the behavior of the cell depends on the physical position of the cell in the two-dimensional space, i.e., on its coordinates), and cellular division (starting from a mother cell, storing the one and only copy of the genome, a new cell can be programmed to store an exact copy of the genome). 


\subsubsection{Self-repair}

In the BIODULE 601 (Figure 3.2), the existence of a fault is decided by the human user by pushing the KILL button of a cell. Therefore, fault detection and fault location, two features which will be indispensable in the final system, where they will be implemented using BIST (Built-In Self-Test) techniques [1,63,103], are not present in the BIODULES 601.

To implement self-repair, we have chosen, favoring simplicity, the following process (Figure 3.2 and Figure 3.3):

$\Rightarrow$ pressing the KILL button determines the faulty cell;

$\Rightarrow$ all the functions of the MICTREE cell are shifted by one cell (or, in the general case, by one column) to the right until the first spare cell (or column) is reached.

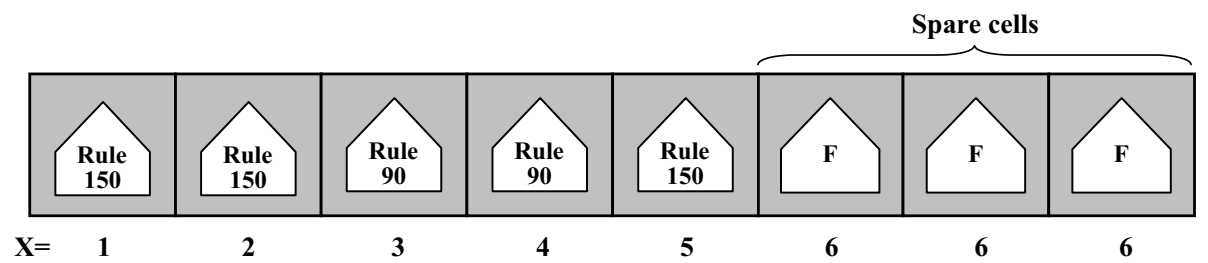

(a)

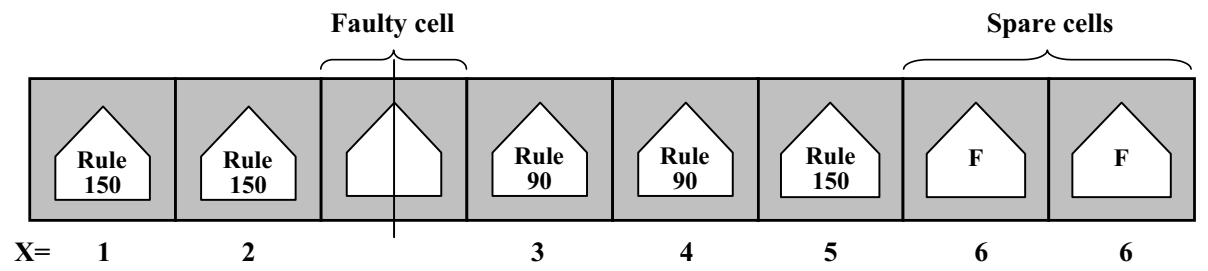

(b)

Figure 3.3: Self-repair of a 5-cell random number generator in an 8-BIODULE 601 array. (a) Original automaton. (b) New automaton after selfrepair.

Obviously, this process requires as many spare cells (or columns), to the right of the array, as there are faulty cells to repair (three spare cells in the example of Figure 3.3, which will be explained in more detail in Section 3.3). It also implies some modifications to the MICTREE cell, so as to add the capability of bypassing the faulty cell and shifting to the right all or part of the original cellular array. 


\subsubsection{Self-replication}

The self-replication of an artificial organism rests on two hypotheses: (1) there exists a sufficient number of spare cells (unused cells at the right hand side of the array, at least five for our example) and (2) the calculation of the coordinates produces a cycle $(X=1 \rightarrow 2 \rightarrow 3 \rightarrow 4 \rightarrow 5 \rightarrow 1 \ldots \rightarrow 5$ in Figure 3.4$)$.

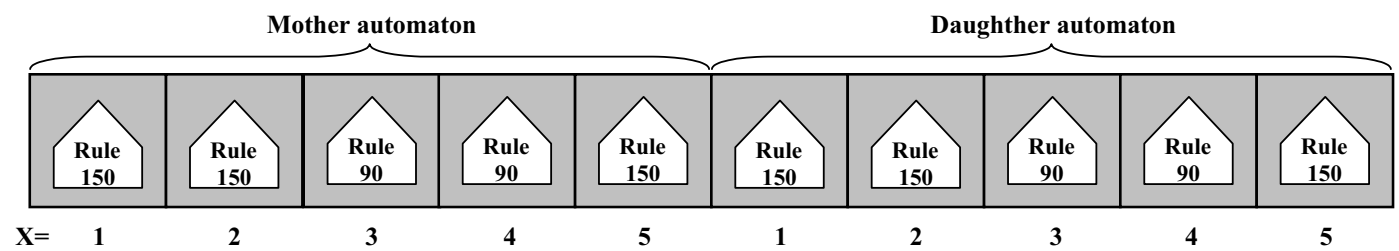

Figure 3.4: Self-replication of a 5-cell random number generator in a 10BIODULE 601 array.

As the same pattern of coordinates produces the same pattern of genes, selfreplication can be easily accomplished if the microprogram of the genome, associated to the homogeneous network of cells, produces several occurrences of the basic pattern of coordinates $(X=1 \rightarrow 2 \rightarrow 3 \rightarrow 4 \rightarrow 5$ in Figure 3.5).

\subsection{Example 1: A random number generator}

In this section we will present a MICTREE implementation of a non-uniform onedimensional cellular automaton (Figure 3.5) that implements a random number generator realized with five cells and featuring two distinct genes (rules 90 and 150 as defined by Wolfram [118]). We will then show that this automaton is capable of self-repair and self-replication.

The development of an artificial organism using MICTREE cells can be broken down into several phases: writing the microcode to compute each one of the genes of the artificial organism, then its coordinates, and finally its complete genome. Each of these phases will be described in the following sub-sections.

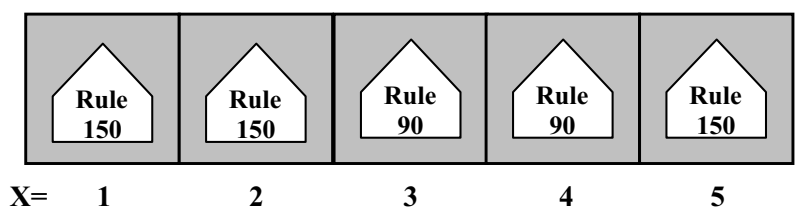

Figure 3.5: Multicellular organization of a random number generator. 


\subsubsection{Description}

Wolfram [118] exhaustively studied one-dimensional cellular automata consisting of identical cells defined by (Figure 3.6):

$\Rightarrow$ a state $Q \in\{0,1\}$

$\Rightarrow$ a neighborhood with a connectivity radius $r=1$, consisting of the state $Q$ of the cell itself, the state $Q W$ of its left-hand (western) neighbor, and the state $Q E$ of its right-hand (eastern) neighbor.

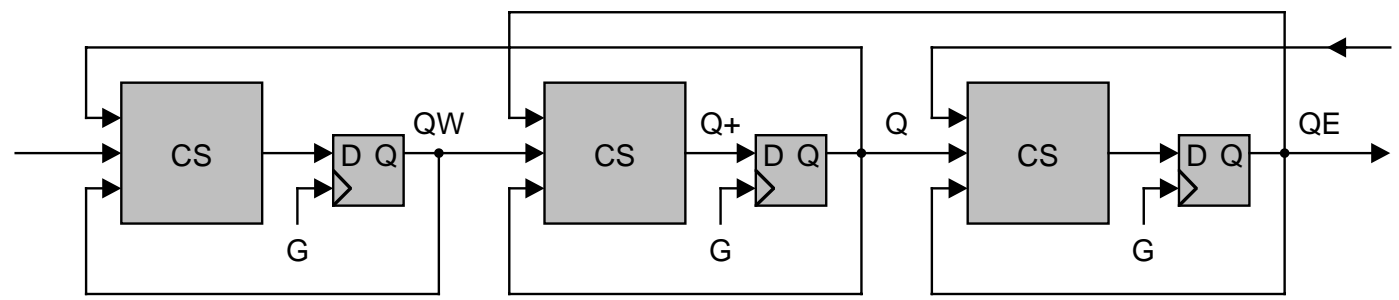

Figure 3.6: Cellular automaton with a 1-bit state per cell and a 3-cell neighborhood $(C S=$ combinational logic system $)$.

\begin{tabular}{|c|ccc|c|c|}
\hline No & QW & $\mathrm{Q}$ & $\mathrm{QE}$ & $\mathrm{Q} 90+=\mathrm{QW} \oplus \mathrm{QE}$ & $\mathrm{Q} 150+=\mathrm{QW} \oplus \mathrm{Q} \oplus \mathrm{QE}$ \\
\hline 0 & 0 & 0 & 0 & 0 & 0 \\
1 & 0 & 0 & 1 & 1 & 1 \\
2 & 0 & 1 & 0 & 0 & 1 \\
3 & 0 & 1 & 1 & 1 & 0 \\
4 & 1 & 0 & 0 & 1 & 1 \\
5 & 1 & 0 & 1 & 0 & 0 \\
6 & 1 & 1 & 0 & 1 & 0 \\
7 & 1 & 1 & 1 & 0 & 1
\end{tabular}

Figure 3.7: Truth table of Wolfram's rules $90(\mathrm{Q} 90+)$ and $150(\mathrm{Q} 150+)$.

The future state $Q+$ of a cell is thus a function of three variables $(Q, Q W$, and $Q E)$, and can be defined by a truth table of $2^{3}=8$ lines (Figure 3.7). There exist $2^{8}=256$ such truth tables, and thus 256 possible functions for a cell of a Wolfram automaton. Each of these functions is called a rule of the automaton. We are interested in the two following rules:

$$
\begin{gathered}
Q+=Q W \oplus Q E=Q W \cdot Q E^{\prime}+Q W^{\prime} \cdot Q E \\
Q+=Q W \oplus Q \oplus Q E
\end{gathered}
$$


where the operation $\oplus$ is the exclusive-OR function, defined in expression (3.4) from the logic operators NOT $\left({ }^{\prime}\right)$, AND $(\cdot)$, and OR $(+)$.

Wolfram identifies each of his rules with the decimal number corresponding to the binary number obtained by reading, from bottom to top, the truth table. Thus, the number corresponding to the rule $R$ of expression (3.4) is obtained as follows (Figure 3.7):

$$
R(Q+=Q W \oplus Q E)=0101 \quad 1010=2^{6}+2^{4}+2^{3}+2^{1}=90
$$

Similarly, for expression (3.5):

$$
R(Q+=Q W \oplus Q \oplus Q E)=1001 \quad 0110=2^{7}+2^{4}+2^{2}+2^{1}=150
$$

Hortensius et al. [31] have shown that a well-chosen arrangement of Wolfram cells of type 90 and 150 produces a non-uniform cellular automaton which is, in fact, a random number generator. For a 5-cell automaton, the final arrangement is shown in Figure 3.8. In such an arrangement, the periodic conditions are equal to 0 :

$$
Q W=0 \quad(\text { for } X=1), Q E=0 \quad(\text { for } X=5)
$$

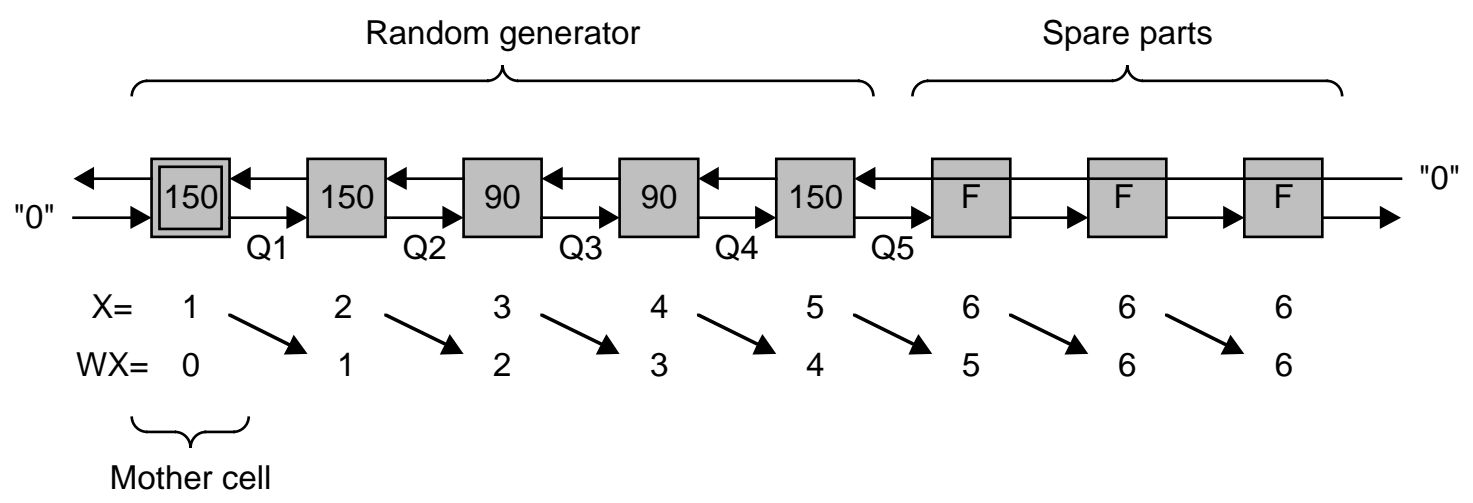

Figure 3.8: One-dimensional 5-cell $(X=1 \ldots 5)$ random number generator with 3 spare cells $(X=6)$; WX: horizontal coordinate of the western neighboring cell.

The global state $Q 1: 5=00000$ is a fixed point of the generator, that is, $Q 1: 5+=00000$. If we suppose that the initial state for this CA is $Q 1: 5=11111$, the remaining $2^{5}-1=31$ states form a cycle of maximal length, defined by the following sequence (in decimal): 


$$
\begin{aligned}
(Q 1: 3, Q 4: 5)= & 73 \rightarrow 20 \rightarrow 70 \rightarrow 32 \rightarrow 43 \rightarrow 72 \rightarrow 23 \rightarrow 62 \rightarrow \\
& 01 \rightarrow 03 \rightarrow 12 \rightarrow 33 \rightarrow 40 \rightarrow 60 \rightarrow 10 \rightarrow 22 \rightarrow \\
& 61 \rightarrow 13 \rightarrow 30 \rightarrow 52 \rightarrow 53 \rightarrow 50 \rightarrow 42 \rightarrow 71 \rightarrow \\
& 31 \rightarrow 51 \rightarrow 41 \rightarrow 63 \rightarrow 02 \rightarrow 11 \rightarrow 21 \rightarrow 73
\end{aligned}
$$

Finally, to demonstrate self-repair, we have decided to add three spare cells to the right of the generator (Figure 3.8). These cells will be identified by the coordinate $X=6$.

For technical reasons, each cell also contains the coordinate $W X$ of its western neighbor. By definition, the leftmost cell, the mother cell, always has coordinates $(W X, X)=(0,1)$.

\subsubsection{Computing the gene for rule 90}

We define a complete (or canonical) binary decision tree as a tree for $n$ variables having $2^{n}$ branches corresponding to the $2^{n}$ possible input states; for the tree of Figure $3.9 \mathrm{~b}$, representing $Q 90+$, we have $n=2$ and $2^{2}=4$. Each test element of the tree is represented by a diamond and defined by a test variable; it has a single input, a true output (test variable equal to 1) and a complemented output (test variable equal to 0 ), identified by a small circle. The leaf elements, represented as squares, define the output values of the given function $(Q 90+$ in our example).

In our case, the use of Karnaugh maps for simplifying trees [47,48] shows that no simplification of $Q 90+$ is possible (Figure 3.9a: there is no block, i.e., no pattern formed by $2^{m}$ adjacent 0 s or $1 \mathrm{~s}$ ). However, joining the common sub-trees (in Figure $3.9 \mathrm{~b}$, the output elements 0 and 1) implies the convergence of some of the tree's branches and thus its transformation into a new representation: the binary decision diagram (BDD) (Figure 3.9c).

The systematic simplification of binary decision diagrams is not a trivial task. For problems of up to 6 variables, Karnaugh maps allow a relatively simple manual computation $[47,48]$. For more than 6 variables, more complex analytical methods are required, and the use of computers becomes necessary $[13,18]$.

For a microprogrammed realization, the binary decision diagram of Figure 3.9c is the flowchart for the gene of rule 90. The software implementation of this flowchart (Figure 3.9d) requires two kinds of instructions: a test instruction and an assignment instruction.

The test instruction is defined by an address $A D R$ and a test variable VAR. Each instruction of this kind has one input and two outputs defining the address of the instruction to be executed next: $A D R+1$ when $V A R=1$ (increment) or $A D R O$ when $V A R=0$ (jump). Thus, the mnemonic of a test instruction is: 


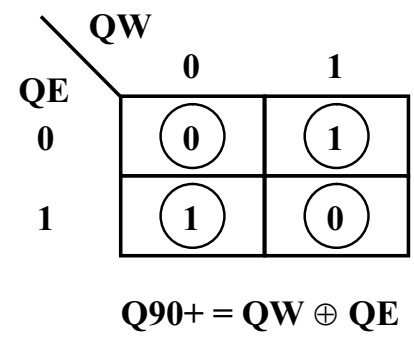

(a)

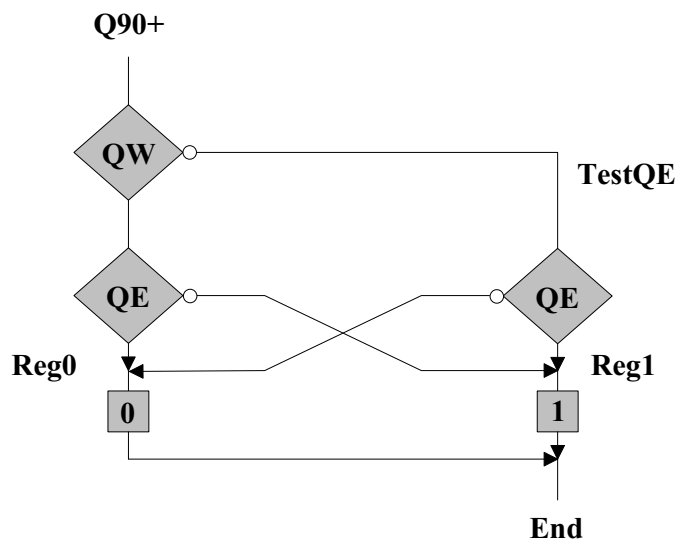

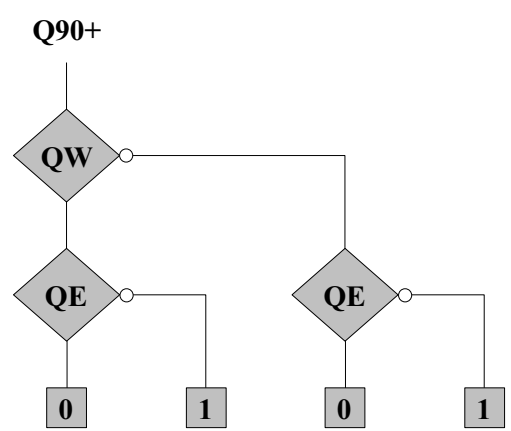

(b)

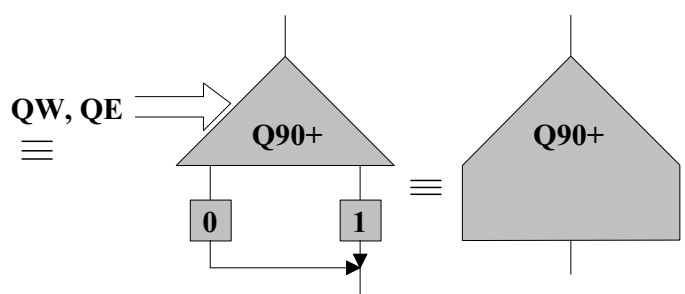

(c)

$\begin{array}{ll}\text { Q90+: } & \text { if } Q W \text { else TestQE } \\ & \text { if } Q E \text { else Reg1 } \\ \text { Reg0: } & \text { do REG }=0 \\ & \text { goto End } \\ \text { TestQE: } & \text { if } Q E \text { else Reg0 } \\ \text { Reg1: } & \text { do REG }=1 \\ \text { End: } & \ldots\end{array}$

(d)

Figure 3.9: Rule 90 gene computation. (a) Karnaugh map. (b) Complete binary decision tree. (c) Binary decision diagram and flowcharts. (d) Assembly language sub-program.

if VAR else $A D R O$

or, in the more agreeable format typical of the assembly language described below:

if VAR else $L A B E L$ 
where $L A B E L$ identifies a unique line in the program, and, therefore, a unique memory address.

The non-conditional jump is a particular case of the test instruction where the test variable is the logic constant 0 . Its mnemonic is simply:

goto $L A B E L$

The assignment instruction is defined by an address $A D R$ and a synchronous assignment $R E G \leftarrow D A T A$, where $R E G$ specifies the cell's register (state register) and DATA an output state. Each instruction of this kind has one input and one output defining the address $A D R+1$ (increment) of the instruction to be executed. The mnemonic of an assignment instruction is:

$$
\text { do } \text { REG = DATA }
$$

The sub-program, or gene, describing rule 90 can then be written using the mnemonics (3.11), (3.12), and (3.13), and the labels Q90+, TestQE, Reg1, Reg0, and End (Figure $3.9 \mathrm{~d})$.

\subsubsection{Computing the gene for rule 150}

On the basis of the definition of the exclusive-OR function and of rule 90 (3.4), the original expression for rule 150 (3.5) can also be written as:

$$
Q 150+=Q \cdot(Q 90+)^{\prime}+Q^{\prime} \cdot(Q 90+)
$$

While no simplification is apparent in the Karnaugh map for $Q 150+$ (Figure 3.10a), we can identify, for $Q=0$, a sub-map (in the form of an outlined block of blocks), equal to the map of function $Q 90+$ of Figure 3.9a.

Transforming the complete binary decision tree derived from Figure 3.10a and joining a number of identical sub-trees (the blocks $S T 1$ and $S T 2$, the output elements 0 and 1), we obtain the binary decision diagram of Figure 3.10b, which is also the flowchart for the gene of rule 150. In accordance with the algebraic expression (3.14), we note that a part of the flowchart is identical to the flowchart for rule $90(Q 90+)$, as shown in Figure 3.9c.

Writing the sub-program implementing rule 150 does not require additional instructions beyond those defined above. 


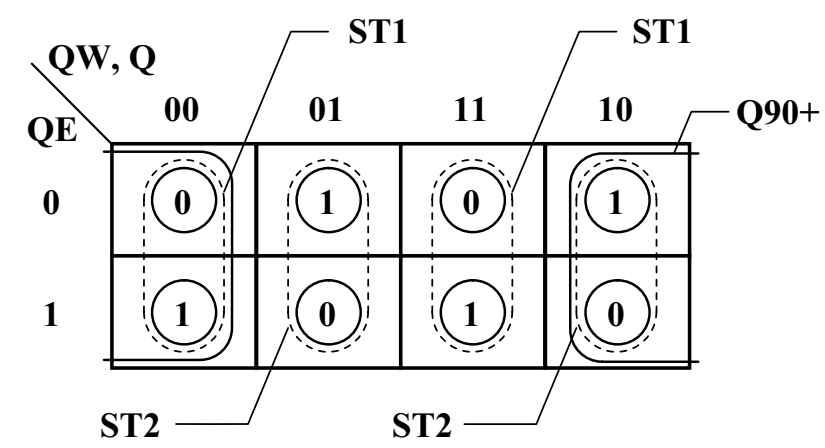

$\mathbf{Q} 150+=\mathbf{Q W} \oplus \mathbf{Q} \oplus \mathbf{Q E}=\mathbf{Q} \cdot\left(\mathbf{Q}^{90+}\right)^{\prime}+\mathbf{Q}^{\prime} \cdot\left(\mathbf{Q}^{90+}\right)$

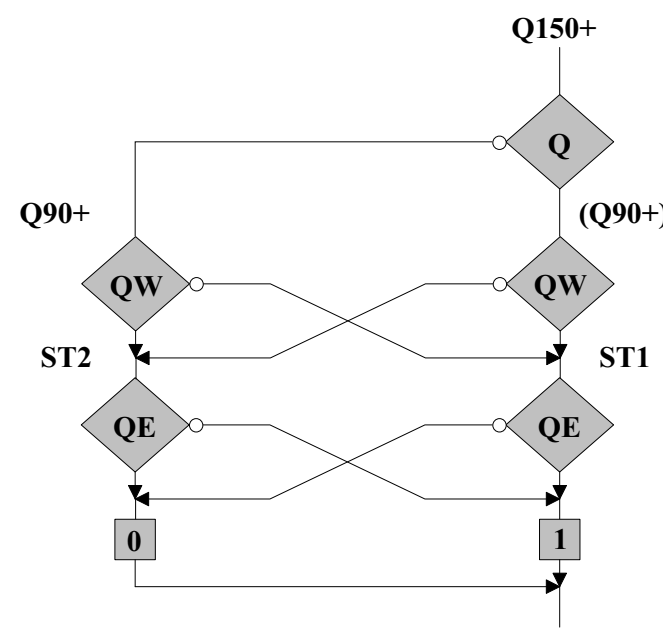

(a)

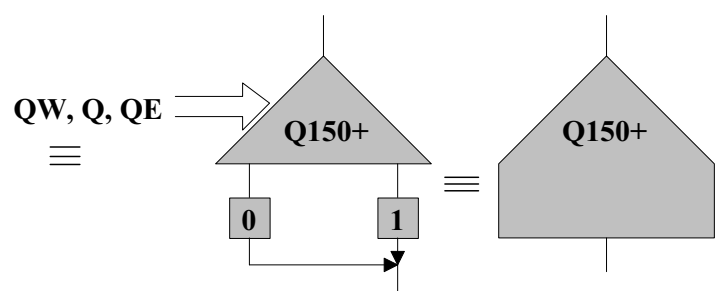

(b)

Figure 3.10: Rule 150 gene computation. (a) Karnaugh map; ST1, ST2: sub-trees.

(b) Binary decision diagram and flowcharts.

\subsubsection{Computing the operational part of the genome}

Cellular differentiation occurs, in our example, only through the horizontal coordinate which, for technical reasons, is computed as a function of the coordinate $W X$ of the preceding cell (the western neighbor). From the description of Figure 3.8, the value of each gene can be described by a 3-variable Karnaugh map. The three variables in question are the three bits $W X 2: 0$ of the horizontal coordinate (Figure 3.11a).

The value $\mathrm{F}$ defines the genes of the spare cells ( $W X=5$ and $W X=6$ ), which display a constant hexadecimal value (equal to F). The simplified binary decision tree derived from Figure 3.11a generates first the binary decision diagram and then the final flowchart for the operational part of the genome, Opgenome, which includes the sub-programs describing the genes $Q 90+, Q 150+$, and $F$ (Figure 3.11b).

Writing the sub-program Opgenome does not require additional instructions beyond those defined above. 


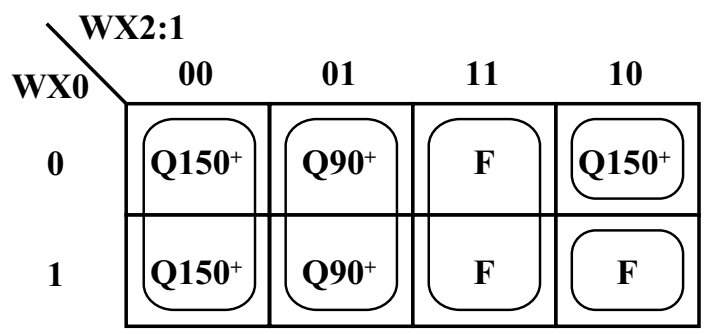

Gene

(a)
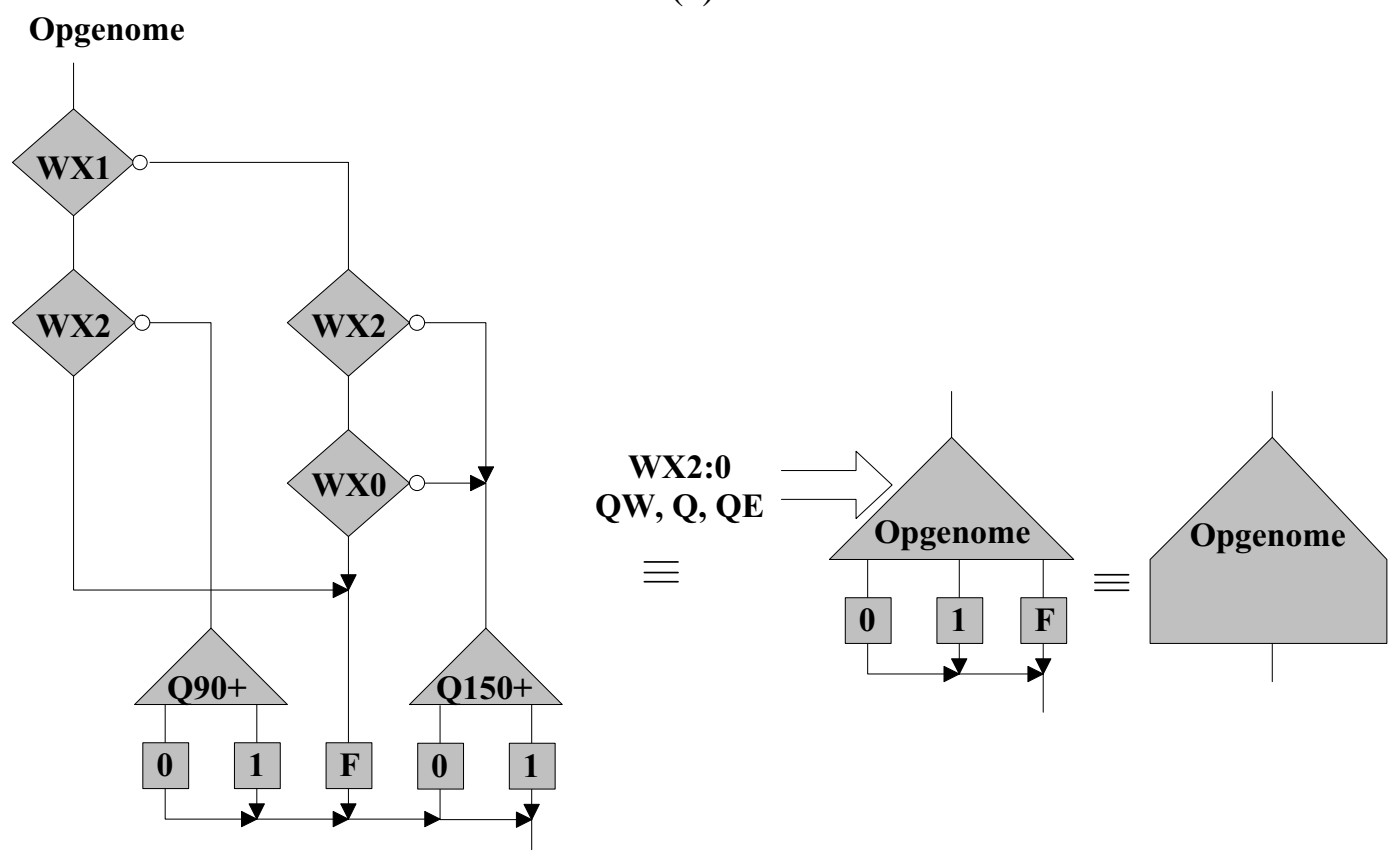

(b)

Figure 3.11: Computing the genome's operational part (sub-program Opgenome). (a) Karnaugh map. (b) Binary decision diagram and flowcharts.

\subsubsection{Computing the coordinates}

The local horizontal coordinate $(X)$ of a given cell is computed as a function of the horizontal coordinate of its western neighbor $(W X)$. If we represent the coordinate $W X$ in its binary form $W X 2: 0$, the specifications of Figure 3.8 allow us to derive directly the Karnaugh map for $X$. A "don't care" condition $\Phi$ (Figure 3.12a) is specified for the (unused) value $W X 2: 0=111$.

The seven blocks of the Karnaugh map generate a simplified binary decision tree with seven branches, which in turn generates a binary decision diagram and, eventually, the flowchart of the sub-program Coord (Figure 3.12b). This flowchart requires the in- 


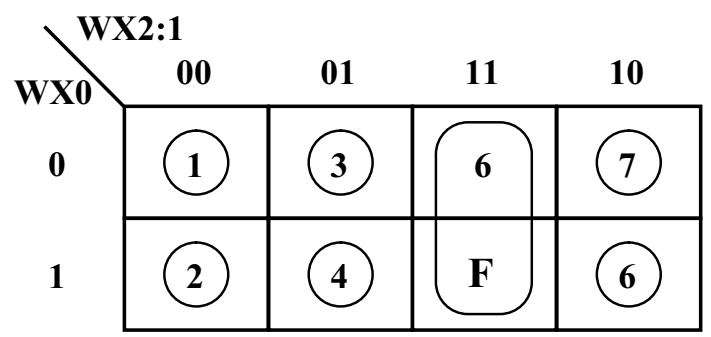

$\mathbf{X}$

(a)
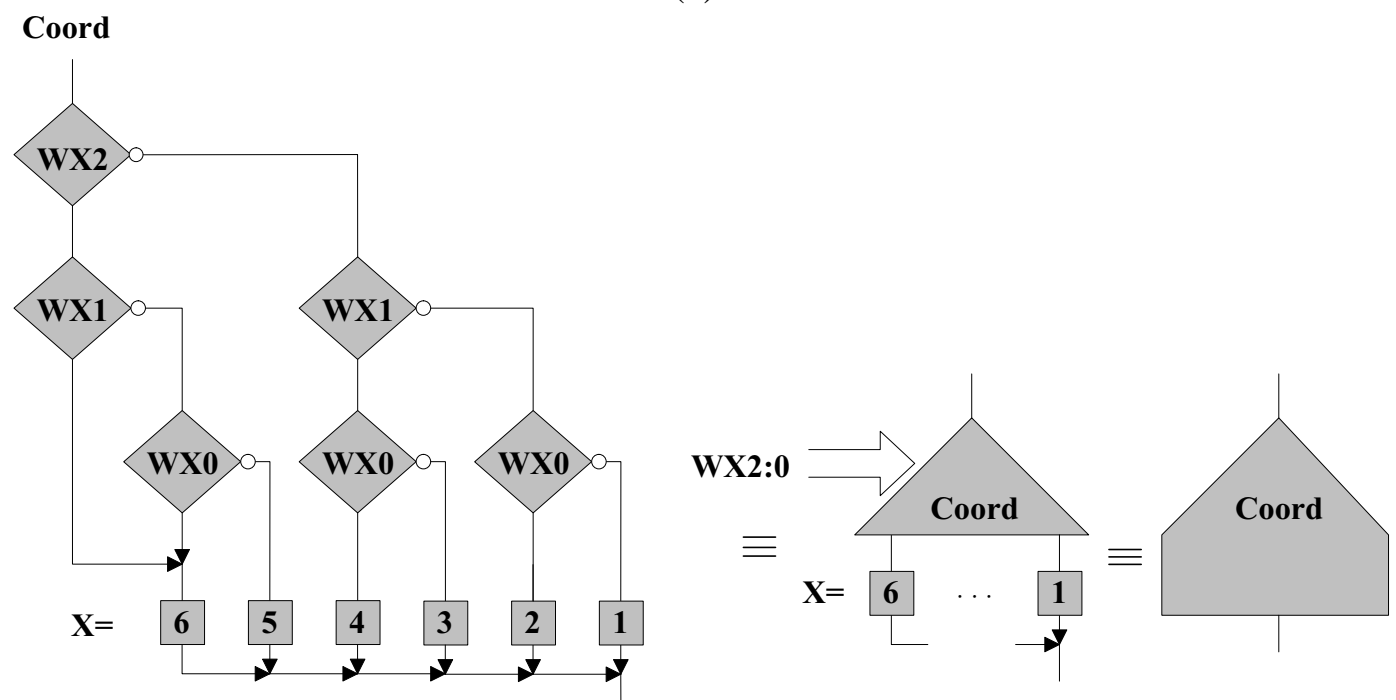

(b)

Figure 3.12: Computing the horizontal coordinate X (sub-program Coord). (a) Karnaugh map. (b) Binary decision diagram and flowcharts.

troduction of a new type of assignment instruction, used exclusively for the computation of the horizontal coordinates. Its mnemonic is:

$$
\text { do } X=D A T A
$$

For the vertical coordinate $Y$, not used in this example, we define a symmetrical assignment instruction whose mnemonic is:

$$
\text { do } Y=D A T A
$$




\subsubsection{Physical configuration}

The original specifications (in particular, Wolfram's rules 90 and 150) allowed us to generate the three genes, realized by the sub-programs Q90+, Q150+, and F. In turn, these genes, differentiated by the address bits $W X 2: 0$, allowed us to generate the sub-program Opgenome, the operational part of the genome. Finally, the sub-program Coord allows each cell to compute its local coordinate $X$ as a function of the coordinate $W X$ of its neighbor. The dynamic part of our microprogram is basically complete.

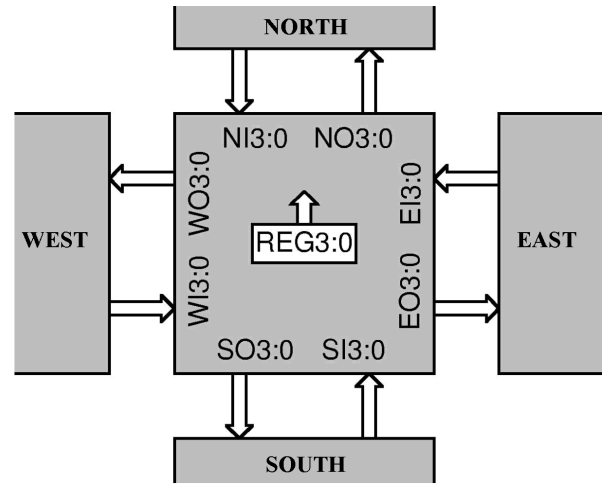

(a)

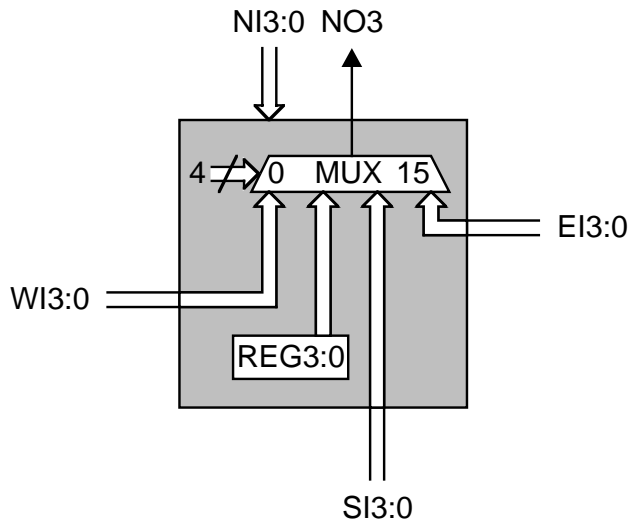

(b)

Figure 3.13: MICTREE cell. (a) Four neighboring cells connection diagram; REG3:0: state register. (b) Output variable NO3 example: 16 different sources programming capability.

We must now define the static part of the final microprogram, which will fix the physical connections between cells and realize the final physical configuration of the device.

Each MICTREE cell (Figure 3.13a) has four neighbors (to the south, west, north, and east). Four 4-bit busses enter the cell from its neighbors (SI3:0 from the south, WI3:0 from the west, NI3:0 from the north, and EI3:0 from the east) and, correspondingly, four output busses go out in the four cardinal directions (SO3:0 to the south, WO3:0 to the west, NO3:0 to the north, and EO3:0 to the east).

Each MICTREE cell has, therefore, 16 outputs SO3...EOO. Each of these outputs can be programmed to take a value from one of 16 possible sources (Figure 3.13b). For example, output $\mathrm{NO} 3$ can take one of the following 16 values:

$\approx$ the four bits $R E G 3: 0$ of register $R E G$;

$\Rightarrow$ the four bits $S I 3: 0$ of the south input bus $S I$;

$\Rightarrow$ the four bits WI3:0 of the west input bus WI; 
$\Rightarrow$ the four bits EI3:0 of the east input bus $E I$.

Note that it is impossible for NO3 to get the value of one of the four bits NI3:0 of the input bus corresponding to the same cardinal direction.

In our assembly language, a single assignment instruction is sufficient to perform this operation. The mnemonic for the instruction is:

$$
\text { do VAROUT }=\text { VARIN }
$$

\subsubsection{Global configuration}

A physical configuration is global when it is realized in all the MICTREE cells of the array, independently of the value of the coordinates ( $X$ and/or $Y$ ).

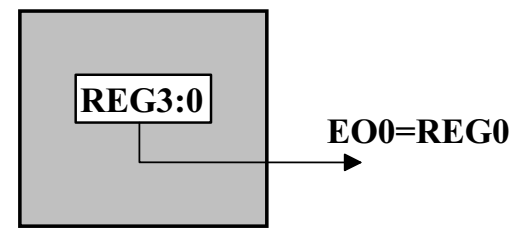

Figure 3.14: Random number generator global configuration.

The diagram of the generator of Figure 3.8 leads us to choose the bit $R E G O$ of the register as the state of the cell $(Q 1 \ldots Q 5)$. From each cell, this value is sent out to the right neighbor through the east output bus $(E O O)$. We thus have the global configuration of Figure 3.14, described by an assignment instruction of type (3.17):

$$
\text { do } E O O=R E G O
$$

The states $R E G O$ of the spare cells $(X=6)$ are not significant and can be shifted to the right without affecting the operation of the array (Figure 3.8).

\subsubsection{Local configuration}

A physical configuration is local if it is realized by a sub-set of the MICTREE cells of the array. Such a configuration depends therefore on the value of the $X$ and/or $Y$ coordinates.

The diagram of the generator of Figure 3.8 shows such a configuration (Figure 3.15a), as: 


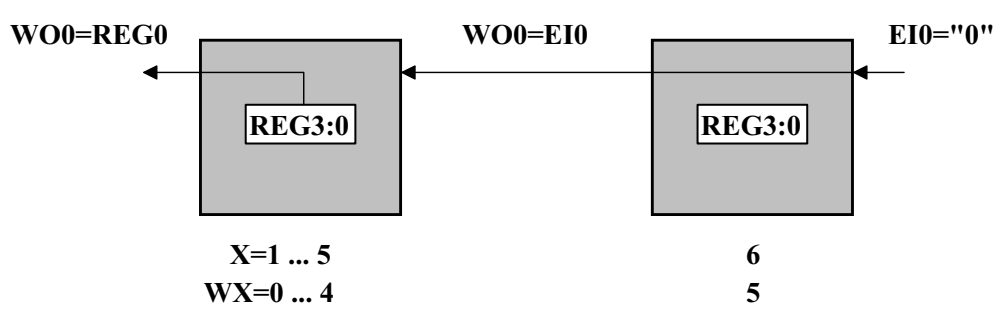

(a)

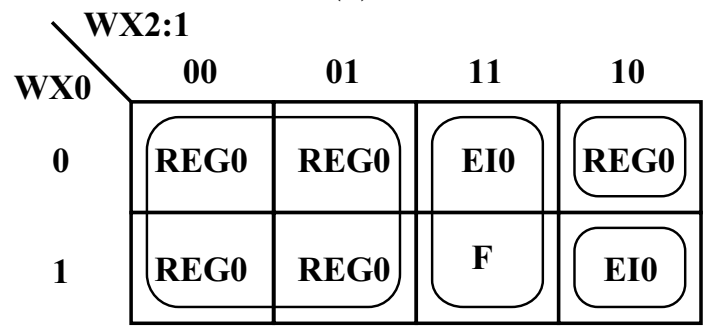

WO0

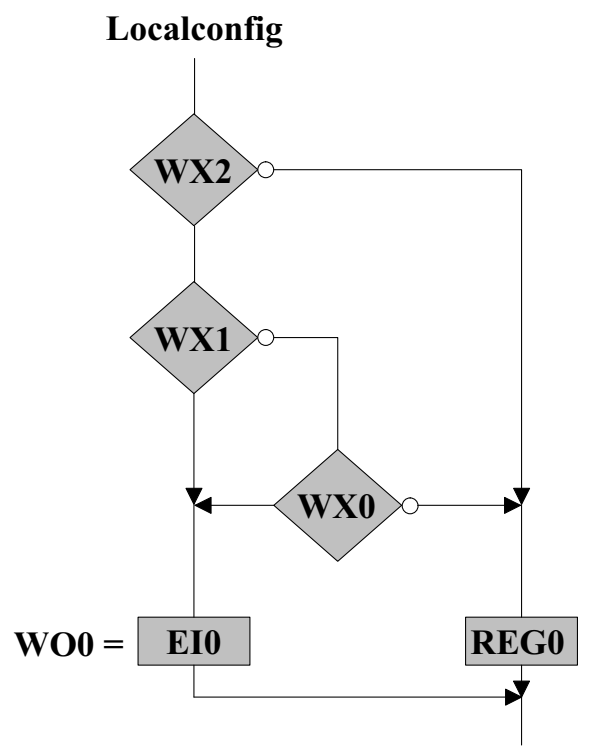

(b)

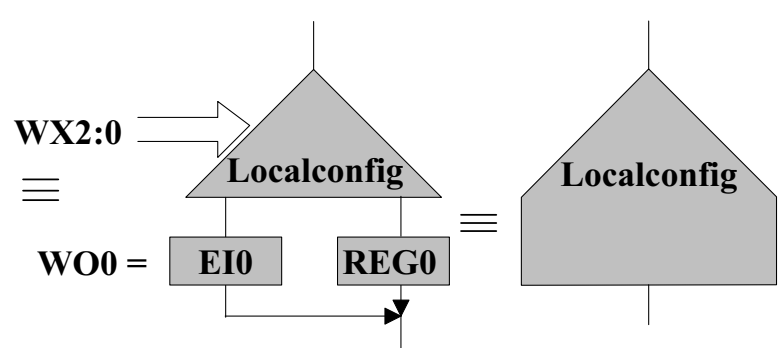

(c)

Figure 3.15: Random number generator local configuration. (a) Block diagram. (b) WOO Karnaugh map. (c) Localconfig sub-program binary decision diagram and flowcharts.

$\Rightarrow$ for $W X=0,1,2,3$, and $4(X=1,2,3,4$, and 5), the state $R E G O$ of the cell must be sent to the cell immediately to the west through the output bus WOO:

$$
\text { do } W O O=R E G O
$$


$\Rightarrow$ for $W X=5$ and $6(X=6)$, the periodic condition, equal to the logic constant 0 , must replace the state $R E G 0$ :

$$
\text { do } W O O=E I O
$$

requiring that the rightmost cell of the array be cabled so as to assure $E I O=0$.

The Karnaugh map of Figure 3.15b describes the local configurations of $W O O$ as a function of the variables $W X 2: 0$ and leads to the flowchart Localconfig of Figure 3.15c. The realization of this flowchart does not require additional instructions beyond those defined above.

\subsubsection{Microprogram of the complete genome}

The complete genome is represented by the final flowchart Generatorgenome of Figure 3.16a. It starts with initial conditions assuring that:

$\Rightarrow$ all the cells of the array are set to $1(R E G O=1)$, which guarantees that the initial state of the generator will be $Q 1: 5=11111$, part of the maximal cycle, and avoids the fixed point $Q 1: 5=00000$;

$\Rightarrow$ the coordinates $X$ and $Y$ are set to $0(X=0, Y=0)$.

The microprogram then executes a double loop, controlled by the variable $G$ (the global clock), the clock signal charged with synchronizing the cellular automaton (Figure 3.6), allowing the transition from the present state $Q 1: 5$ to the future state $Q 1: 5+$ at each rising edge of $G(G=0 \rightarrow 1)$.

The right-hand loop is executed once every period (of duration $t G$ ) of the global clock signal $G$. In this loop, the operational part of the genome (Opgenome) is also executed once. To assure the synchronization of all the cells, tests are performed throughout the half-period where $G=0$, but no assignment is made until the rising edge of $G$ $(G=0 \rightarrow 1$ ), when the registers $R E G$ (i.e. the states of the cells) are updated and, for security, the global configuration is confirmed (Globalconfig).

If $K$ is the number of used cells ( $K=8$ in the example), the left-hand loop must be executed at least 8 times during the half-period when $G=1$ (Figure 3.16b). At the start of the microprogram, or when a repair involving a change of coordinates occurs, the coordinates are recomputed starting from the left-most cell (the mother cell, with $W X=0$ ). At least $K$ executions of the left-hand loop are necessary to ensure that the right-most cell computes the correct coordinates. This computation occurs in the sub-program Coord, which is immediately followed by the computation of the local configurations (Localconfig). If tex is the total execution time of the Coord and Localconfig sub-programs, we must verify that the following inequality holds (Figure 3.16b): 


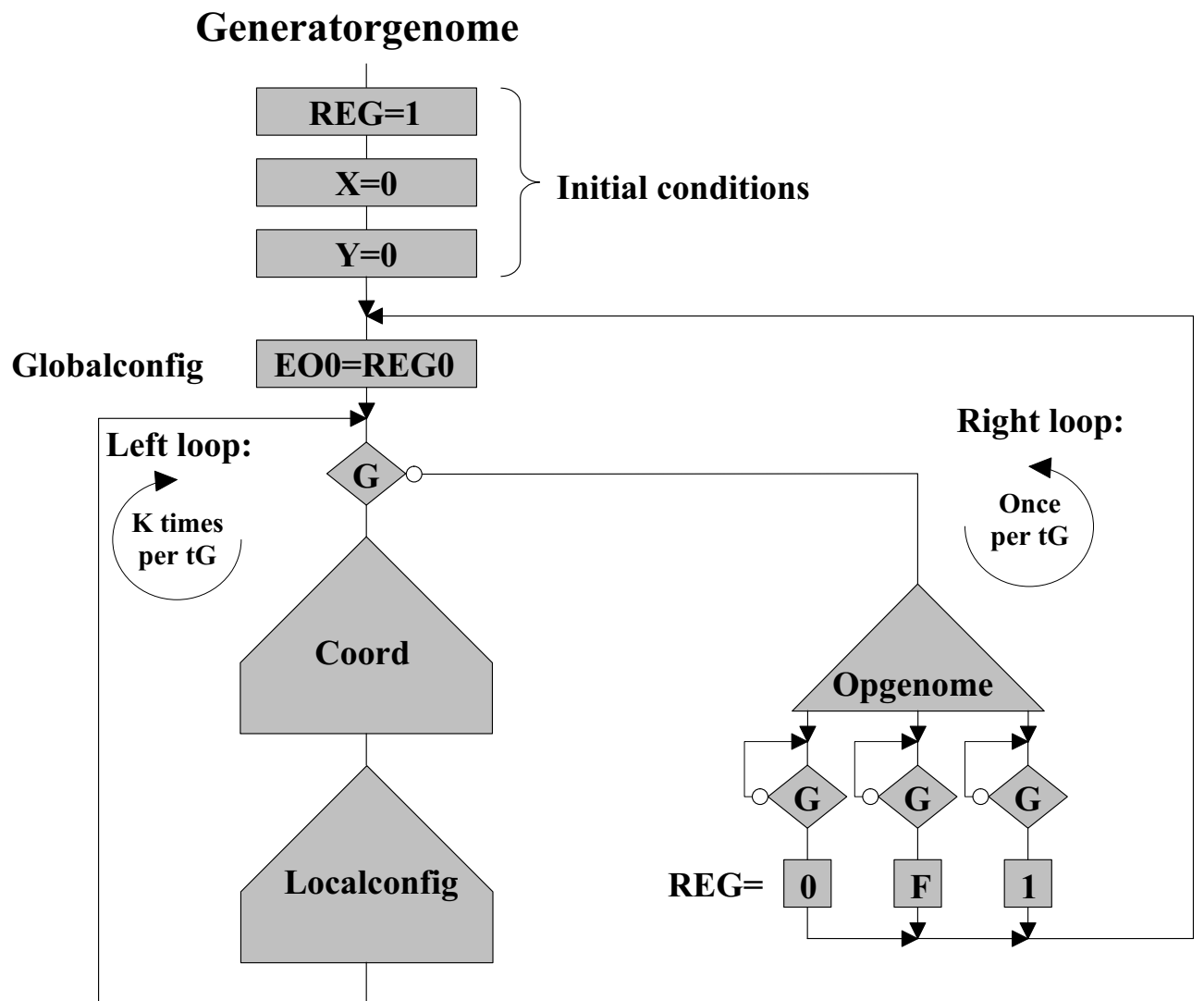

(a)

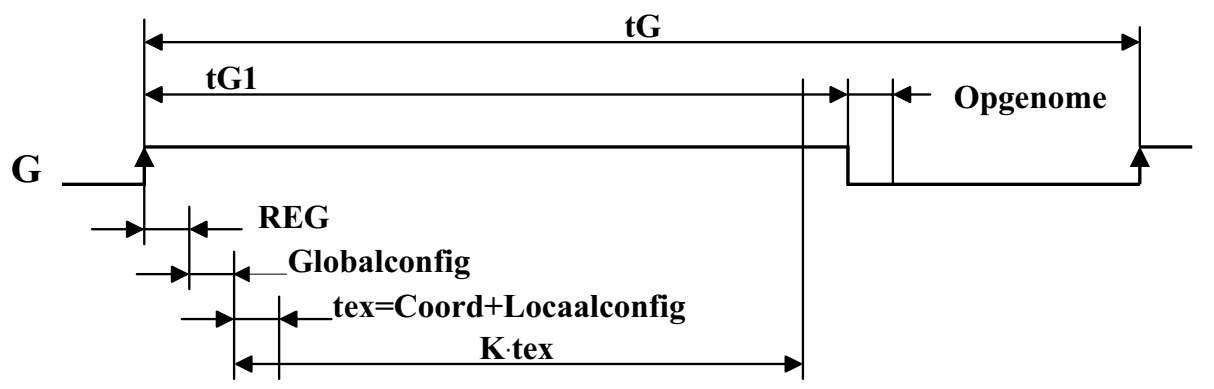

(b)

Figure 3.16: Complete genome microprogram (Generatorgenome). (a) Flowchart. (b) Timing diagram; G: global clock.

$$
K \cdot \operatorname{tex}<t G 1
$$

where $t G 1$ is the half-period of $G$ when $G=1$ (Figure 3.16b). In this expression, we consider negligible the time of execution of the assignment instructions for REG and Globalconfig. 


\subsection{Example 2: A 2-by-n hybrid cellular automaton}

2-by-n (2-by-n arrays of cells) cellular automata (CA) are a subset of regular twodimensional linear CA. In these automata each cell is connected only to its immediate neighbors (connectivity radius $r=1$ ), and there are three such neighbors for each cell. The leftmost and rightmost cells are assumed to have a constant -0 input. The state $Q$ of a cell is calculated using the cell's rule. Different cells can use different rules, making the CA hybrid [33]. Figure 3.17 shows an example of a 2-by-3 cellular automaton with a spare column.

This 2-by-3 cellular automaton (with a spare column) has been completely simulated [86, 89], using MIC Sim [116, 117] (Sub-Section 3.5.3), and implemented with our MICTREE artificial cells. Both the simulation and the physical implementation verified the theoretical results.

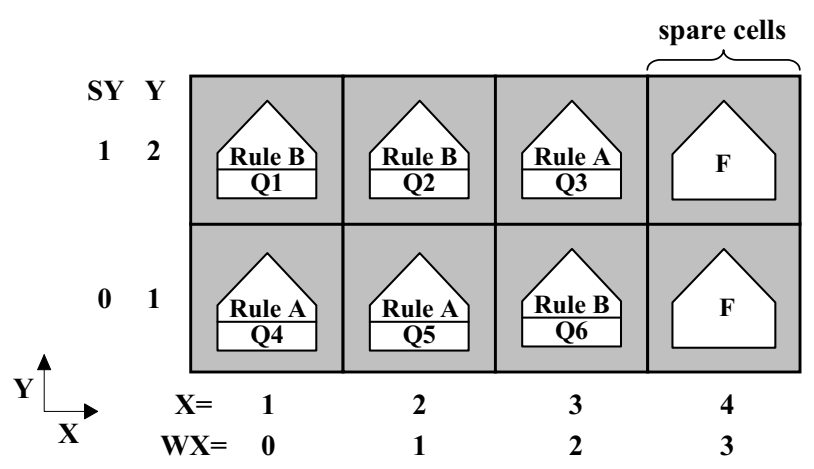

Figure 3.17: Two-dimensional 2-by-3 cellular automaton with a spare column.

This CA presents two rules, which are natural generalizations of Wolfram's rules 90 and 150. The future state $Q+$ of a cell is a function of four variables $(Q, Q W, Q E, Q V)$

$$
\begin{gathered}
Q A+=f(Q, Q W, Q E, Q V)=Q W \oplus Q E \oplus Q V \\
Q B+=f(Q, Q W, Q E, Q V)=Q W \oplus Q E \oplus Q V \oplus Q
\end{gathered}
$$

where the operator $\oplus$ denotes the exclusive-OR function, $Q W, Q E$, and $Q V$ denote the present state of the west, east, and vertical neighbor respectively, and $Q$ denotes the present state of the cell itself.

The global state $Q 1: 6=000000$ is a fixed point of the automaton, that is, $Q 1: 6+=000000$. If we suppose that the initial state for this CA is $Q 1: 6=111111$, the remaining $2^{6}-1=63$ states form a cycle of maximal length, defined by the following sequence (in decimal) [33]: 


$$
\begin{aligned}
(Q 1: 3, Q 4: 6)= & 77 \rightarrow 43 \rightarrow 52 \rightarrow 60 \rightarrow 16 \rightarrow 46 \rightarrow 03 \rightarrow 36 \rightarrow \\
& 34 \rightarrow 11 \rightarrow 32 \rightarrow 76 \rightarrow 50 \rightarrow 45 \rightarrow 35 \rightarrow 02 \rightarrow \\
& 25 \rightarrow 23 \rightarrow 44 \rightarrow 26 \rightarrow 15 \rightarrow 70 \rightarrow 37 \rightarrow 27 \rightarrow \\
& 06 \rightarrow 67 \rightarrow 62 \rightarrow 33 \rightarrow 65 \rightarrow 47 \rightarrow 10 \rightarrow 21 \rightarrow \\
& 61 \rightarrow 05 \rightarrow 51 \rightarrow 56 \rightarrow 22 \rightarrow 57 \rightarrow 31 \rightarrow 40 \rightarrow \\
& 64 \rightarrow 54 \rightarrow 07 \rightarrow 74 \rightarrow 75 \rightarrow 66 \rightarrow 71 \rightarrow 24 \rightarrow \\
& 30 \rightarrow 53 \rightarrow 73 \rightarrow 01 \rightarrow 13 \rightarrow 17 \rightarrow 55 \rightarrow 14 \rightarrow \\
& 63 \rightarrow 20 \rightarrow 72 \rightarrow 12 \rightarrow 04 \rightarrow 42 \rightarrow 41 \rightarrow 77
\end{aligned}
$$

\subsubsection{Genes, coordinates, and genome}

To calculate the complete genome of this CA we need to calculate first each gene of the artificial organism (rules A and B), then its coordinates, and finally the local and global configurations.

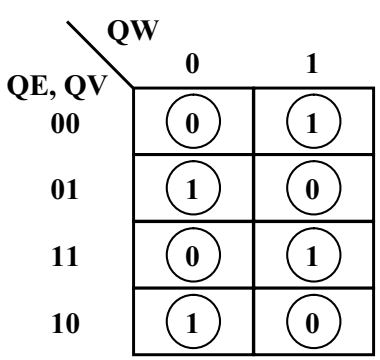

$\mathbf{Q A}+=\mathbf{Q W} \oplus \mathbf{Q E} \oplus \mathbf{Q V}$

(a)
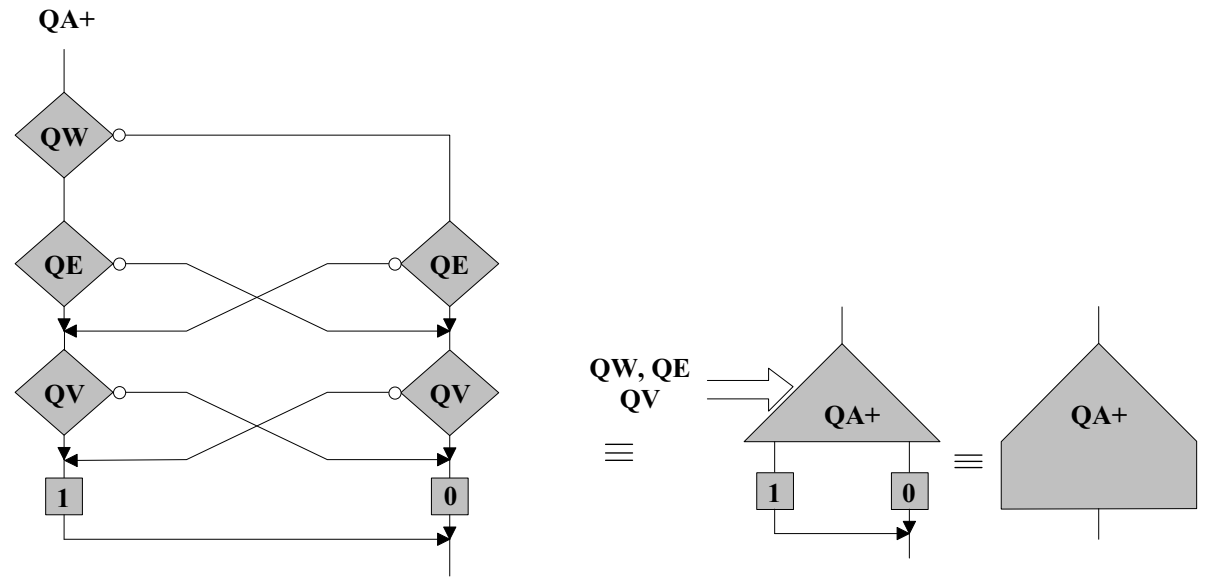

(b)

Figure 3.18: Rule A gene computation. (a) Karnaugh map. (b) Binary decision diagram and flowcharts.

The future state $Q A+$ is represented by the Karnaugh map of Figure 3.18a. This Karnaugh map shows that no simplification of $Q A+$ is possible (there is no block, i.e., no 
pattern formed by $2^{m}$ adjacent 0 s or $1 \mathrm{~s}$ ). For a microprogrammed realization, the binary decision diagram of Figure $3.18 \mathrm{~b}$ is the flowchart for the gene of rule A.

While no simplification is apparent in the Karnaugh map for $Q B+($ Figure 3.19a), we can identify, for $Q=0$, a sub-map (in the form of an outlined block of blocks), equal to the map of function $Q A+$ of Figure 3.18a.

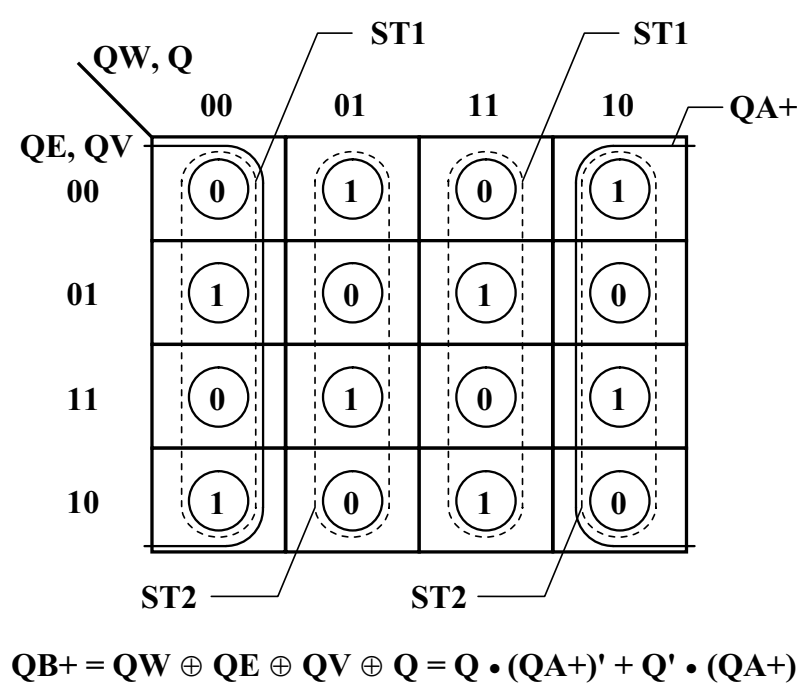

(a)
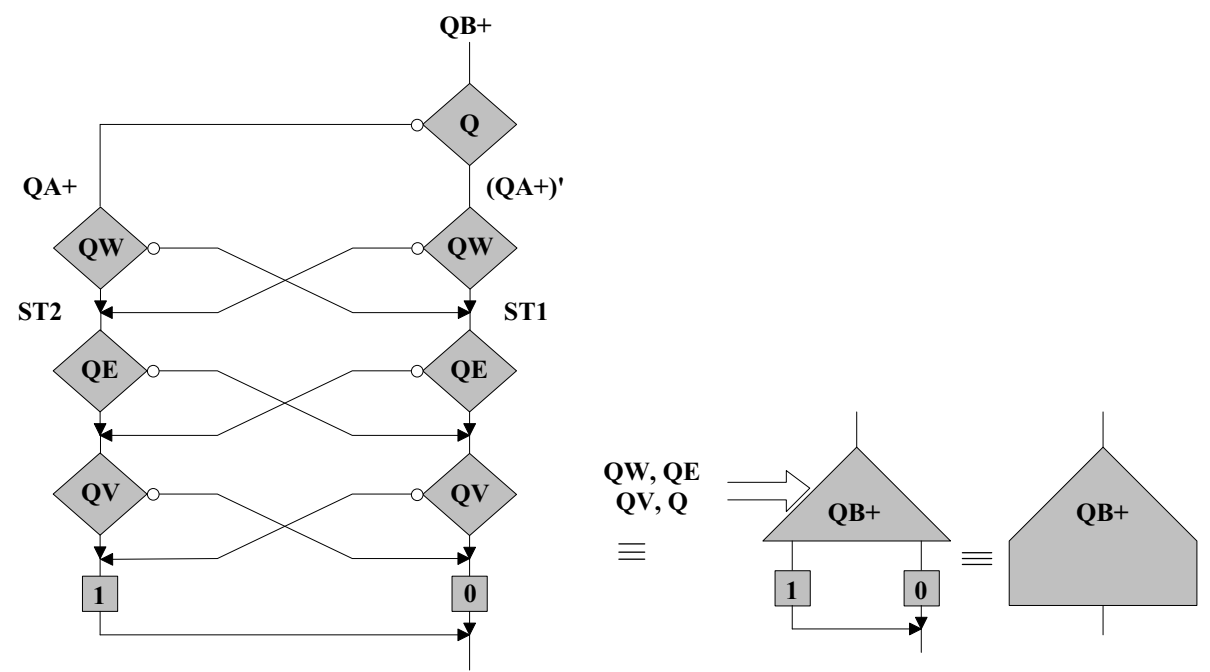

(b)

Figure 3.19: Rule B gene computation. (a) Karnaugh map. (b) Binary decision diagram and flowcharts.

Transforming the complete binary decision tree derived from Figure 3.19a and joining a number of identical sub-trees (the blocks $S T 1$ and $S T 2$, the output elements 0 and 1), we obtain the binary decision diagram of Figure 3.19b, which is also the flowchart for the gene of rule B. In accordance with the algebraic expression (3.23), we note that a part of the flowchart is identical to the flowchart for rule $\mathrm{A}(Q A+)$, as shown in Figure 3.19b. 
Cellular differentiation occurs, in our example, through the horizontal and vertical coordinates. From the description of Figure 3.17, the value of each gene can be described by a 3-variable Karnaugh map. The three variables in question are the two bits WX1:0 of the horizontal coordinate and the bit $S Y O$ of the vertical coordinate (Figure 3.20a).

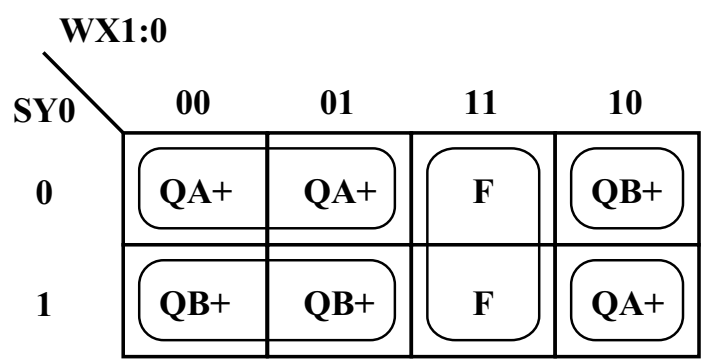

Gene

(a)

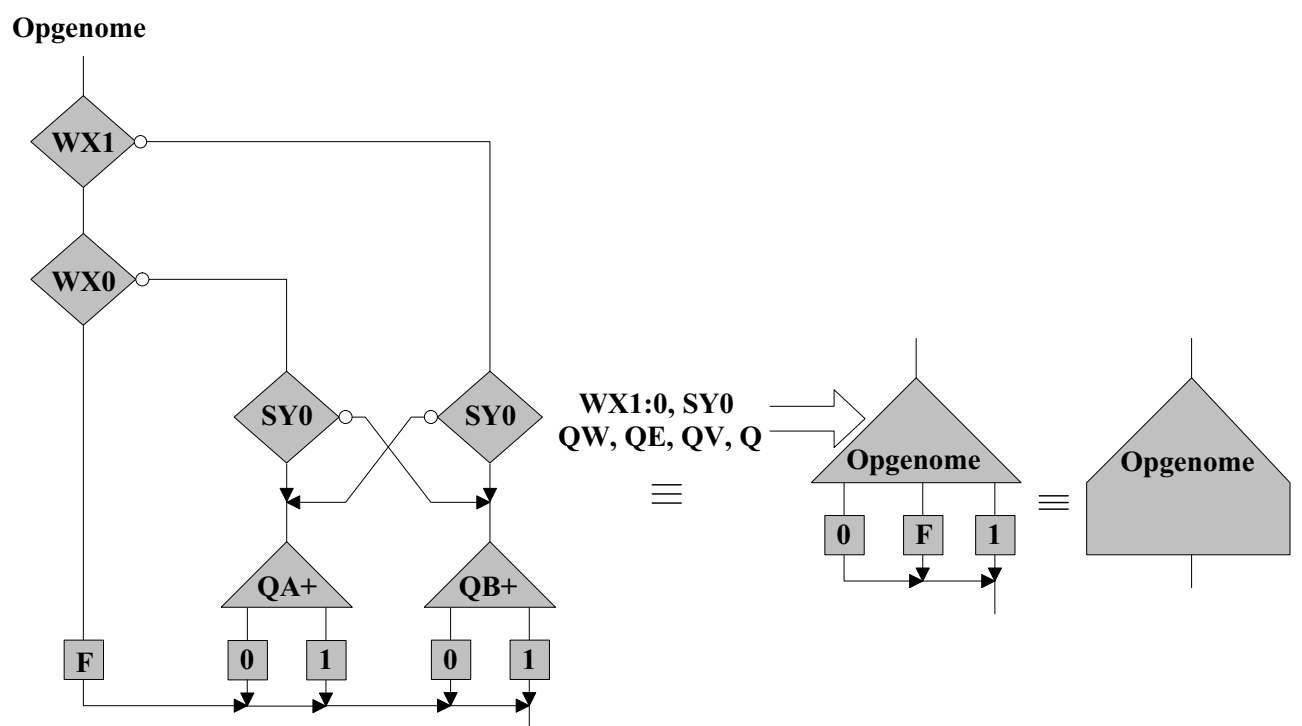

(b)

Figure 3.20: Computing the genome's operational part (sub-program Opgenome). (a) Karnaugh map. (b) Binary decision diagram and flowcharts.

The value $\mathrm{F}$ defines the gene of the spare cells in the column $(W X=3)$, which display a constant hexadecimal value (equal to $F$ ). The simplified binary decision tree derived from Figure 3.20a generates first the binary decision diagram and then the final flowchart for the operational part of the genome, Opgenome, which includes the sub-programs describing the genes $Q A+, Q B+$, and F (Figure 3.20b).

The Karnaugh map of Figure 3.21a expresses the horizontal coordinate $X$ of a cell as a function of the two individual bits $W X 1: 0$ of the horizontal coordinate $W X$ of the west neighboring cell. The four blocks of the map define a complete binary decision tree that becomes the flowchart of the sub-program Coord (Figure 3.21b). The vertical coordinate $Y$ of a cell is expressed as a function of the bit $S Y O$, thus the use of a Karnaugh map is not 
necessary. The addition of a single test element operating on $S Y O$ will allow the definition of the $Y$ coordinate. The binary decision tree, and the flowchart of the sub-program are showed in Figure 3.21c.

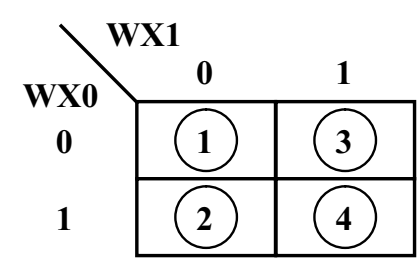

$\mathbf{X}$

(a)
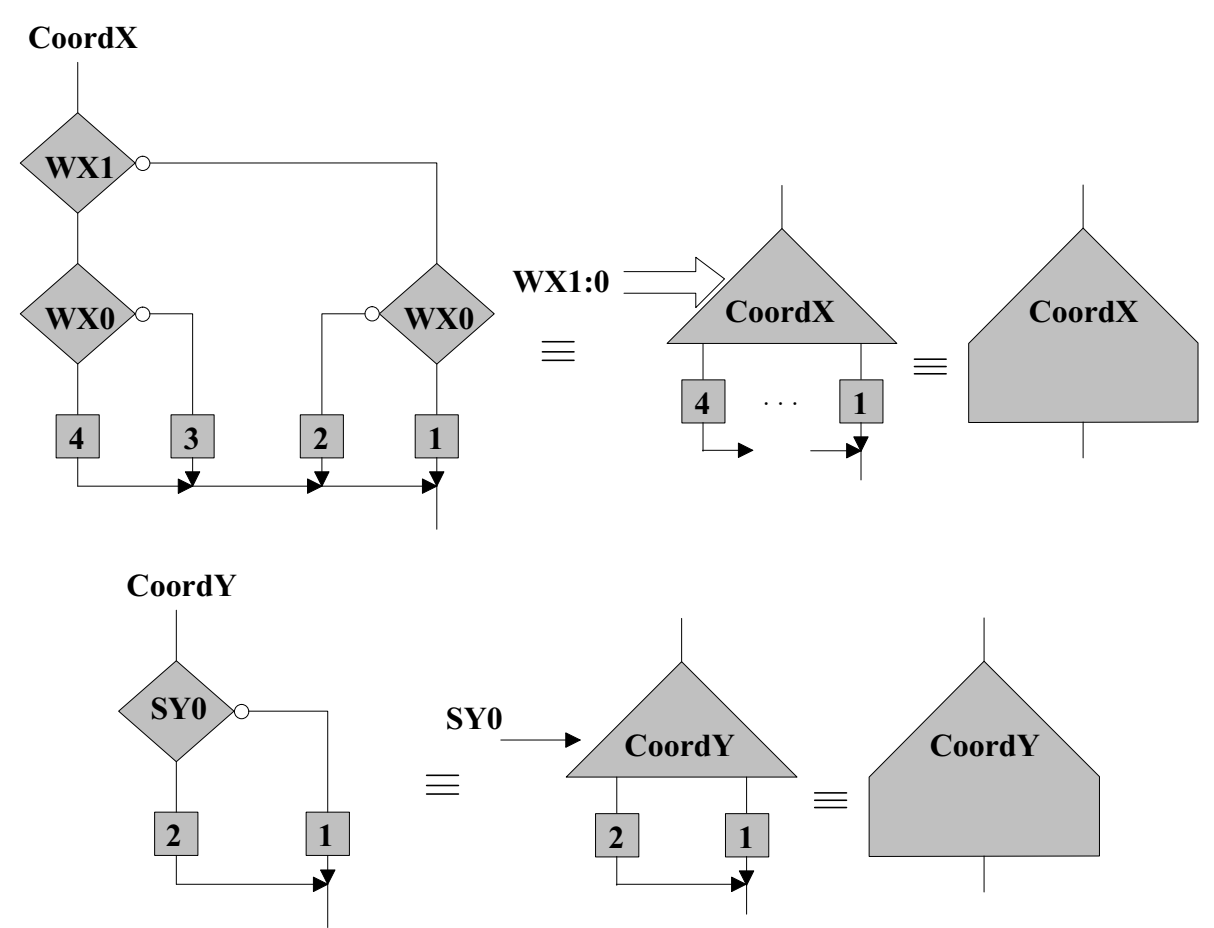

(b)

Figure 3.21: Computing the horizontal and vertical coordinates $X, Y$. (a) Karnaugh map for $X$. (b) Binary decision diagram and flowcharts for $X$ and $Y$ coordinates respectively.

\subsubsection{Configurations and microprogram}

A physical configuration is global when it is realized in all the MICTREE cells of the array, independently of the value of the coordinates ( $X$ and/or $Y$ ).

The diagram of the automaton of Figure 3.17 leads us to choose the bit $R E G 0$ of the register as the state of the cell $(Q 1 \ldots Q 6)$. From each cell, this value is sent out to the right, 
upper, and bottom neighbors through the east, north, and south output busses respectively (EOO, NOO, SOO). We thus have the global configuration of Figure 3.22.

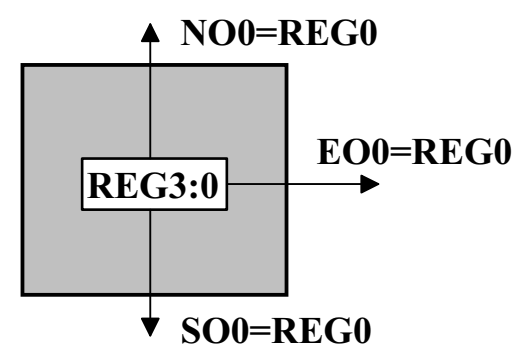

Figure 3.22: 2-by-n CA global configuration.

A physical configuration is local if it is realized by a sub-set of the MICTREE cells of the array. Such a configuration depends therefore on the value of the $X$ and/or $Y$ coordinates.

The diagram of Figure 3.17 shows such a configuration (Figure 3.23b), as:

$\Rightarrow$ for $W X=0,1$, and $2(X=1,2$, and 3), the state $R E G 0$ of the cell must be sent to the cell immediately to the west through the output bus WOO.

$\Rightarrow$ for $W X=3(X=4)$, the periodic condition, equal to the logic constant 0 , must replace the state $R E G 0$, requiring that the rightmost cell of the array be cabled so as to assure $E I O=0$.

The Karnaugh map of Figure 3.23a describes the local configurations of WOO as a function of the variables $W X 1: 0$ and leads to the flowchart Localconfig of Figure 3.23c.

The complete genome is represented by the final flowchart 2-by-3-CA-genome of Figure 3.24a. It starts with initial conditions assuring that:

$\Rightarrow$ all the cells of the array are set to $1(R E G O=1)$, which guarantees that the initial state of the automaton will be $Q 1: 6=111111$, part of the maximal cycle, and avoids the fixed point $Q 1: 6=000000$;

$\Rightarrow$ the coordinates $X$ and $Y$ are set to $0(X=0, Y=0)$.

The microprogram then executes a double loop, controlled by the variable $G$ (the global clock), the clock signal charged with synchronizing the cellular automaton, allowing the transition from the present state $Q 1: 6$ to the future state $Q 1: 6+$ at each rising edge of $G(G=0 \rightarrow 1)$. 


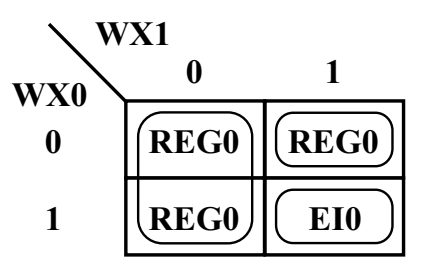

WO0

(a)

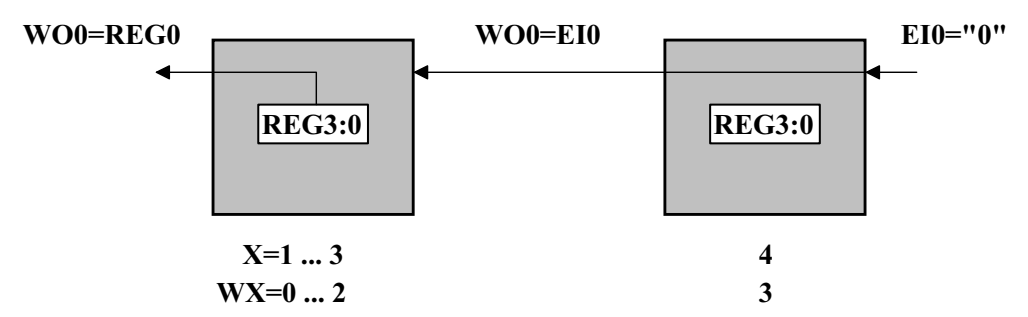

(b)

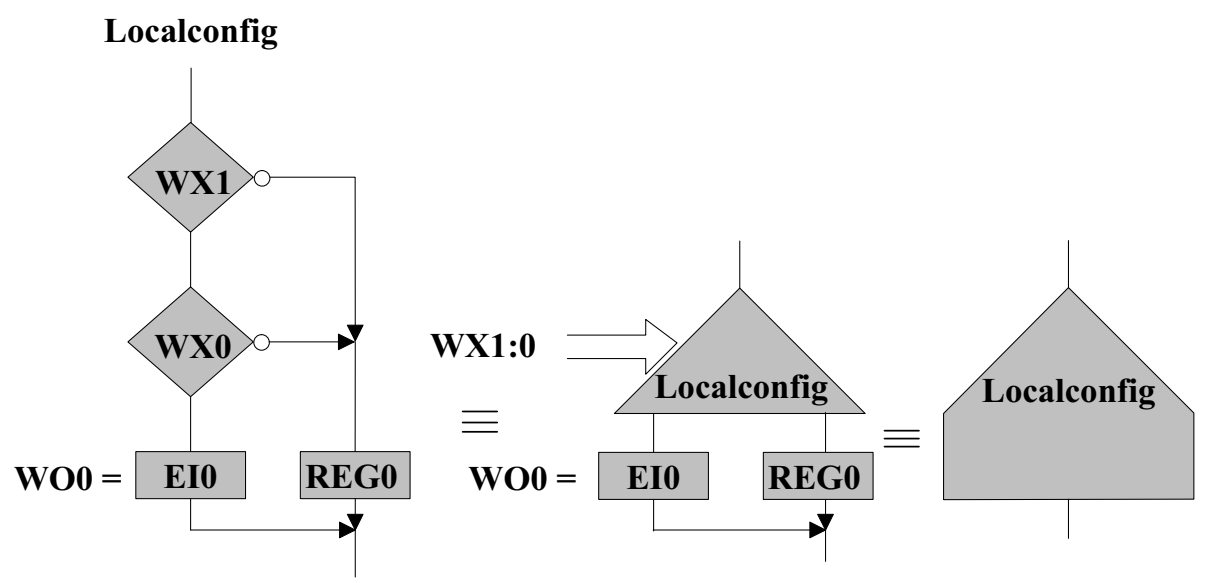

(c)

Figure 3.23: 2-by-n CA local configuration. (a) Karnaugh map. (b) Block diagram. (c) Localconfig sub-program binary decision diagram and flowcharts.

The right-hand loop is executed once every period (of duration $t G$ ) of the global clock signal $G$. In this loop, the operational part of the genome (Opgenome) is also executed once. To assure the synchronization of all the cells, tests are performed throughout the half-period where $G=0$, but no assignment is made until the rising edge of $G$ ( $G=0 \rightarrow 1$ ), when the registers $R E G$ (i.e. the states of the cells) are updated and, for security, the global configuration is confirmed (Globalconfig).

If $K$ is the number of used cells by row ( $K=4$ in the example), the left-hand loop must be executed at least 4 times during the half-period when $G=1$ (Figure 3.24b). At the start of the microprogram, or when a repair involving a change of coordinates occurs, 


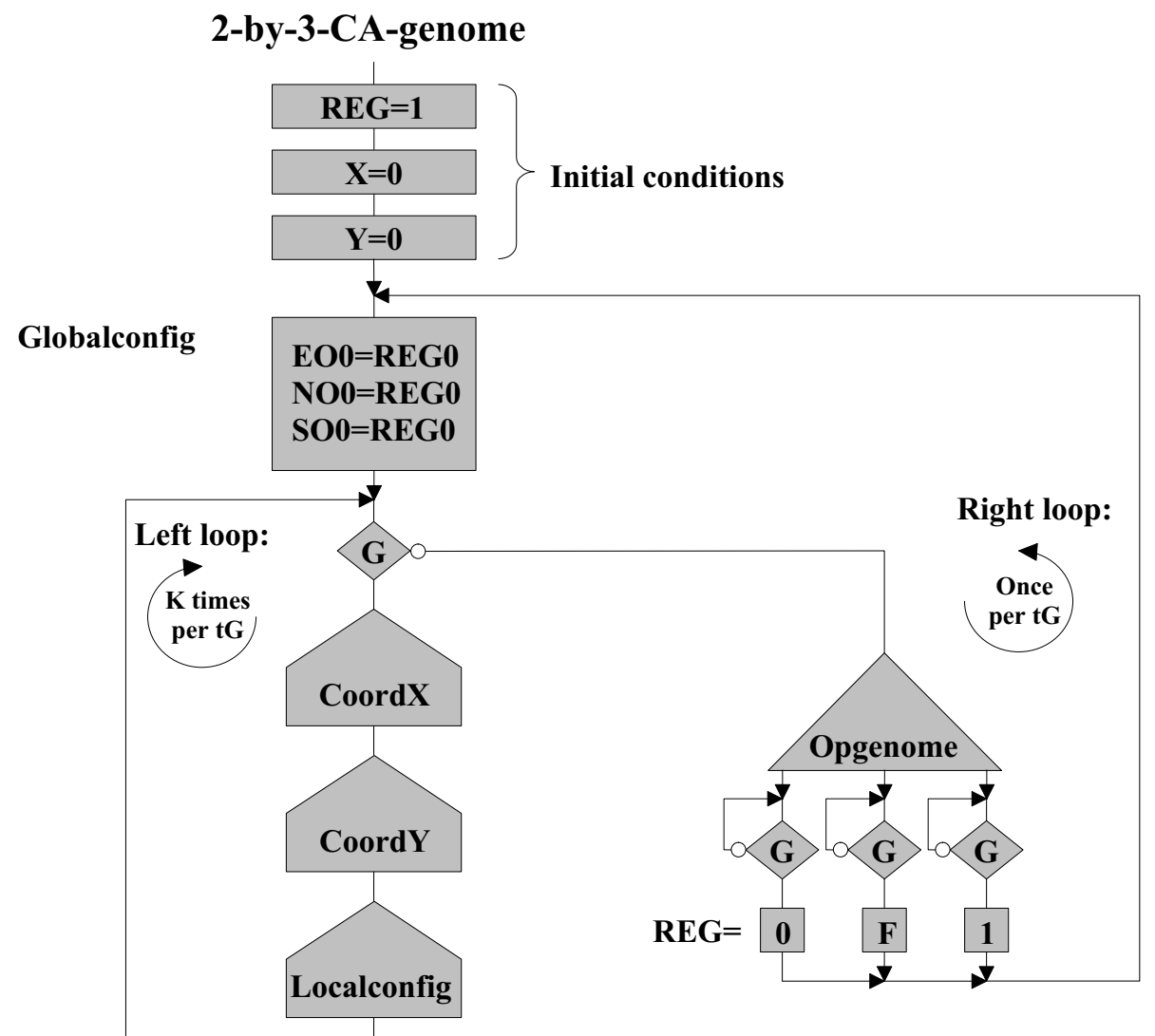

(a)

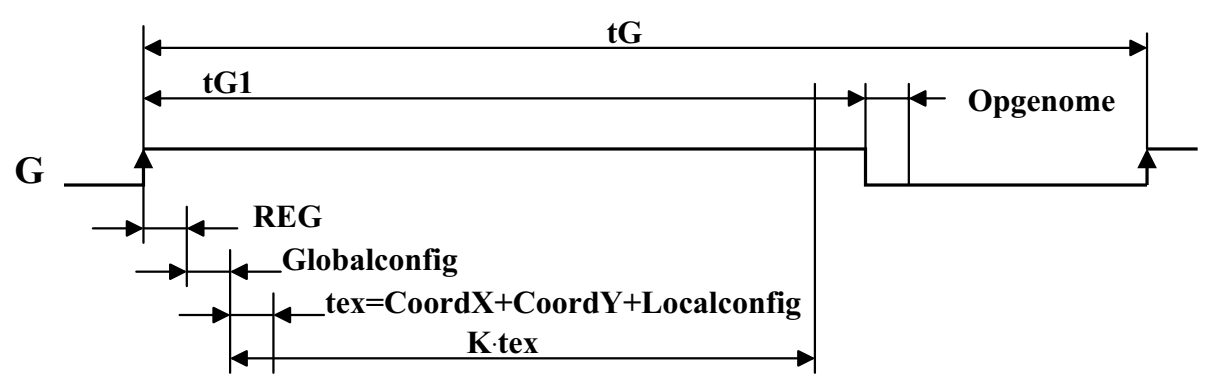

(b)

Figure 3.24: Complete genome: 2-by-3-CA-genome microprogram. (a) Flowchart. (b) timing diagram; G: Global clock.

the coordinates are recomputed starting from the left-most cell (the mother cell, with $W X=0$ ). At least $K$ executions of the left-hand loop are necessary to ensure that the right-most cell computes the correct coordinates. This computation occurs in the subprograms CoordX, and CoordY which are immediately followed by the computation of the local configurations (Localconfig). If tex is the total execution time of the CoordX, CoordY, and Localconfig sub-programs, we must verify that the following inequality holds (Figure 3.24b): 


$$
K \cdot \operatorname{tex}<t G 1
$$

where $t G 1$ is the half-period of $G$ when $G=1$ (Figure 3.24b). In this expression, we consider negligible the time of execution of the assignment instructions for REG and Globalconfig.

\subsection{Software tools}

As we could see from the examples in Sections 3.3 and 3.4, the MICTREE cells can be used to create complex artificial organisms, but the design of the software programs defining their behavior is difficult. In this section we present a short description of the different software tools that have been developed to deal with the programming complexity of our artificial cells (a detailed description can be found in Chapter 6). All of this software tools have been developed for the Windows operating system.

\subsubsection{MIC Editor and MIC Compiler}

MIC Editor is a general-purpose, easy-to-use, text editor for the MIC language (the language used to program the MICTREE artificial cell). It also offers many powerful features for MIC programmers, such as syntax highlighting, dedicated toolbars, line numbering, search and replace, keyboard shortcuts, and more.

The MIC Compiler, which integrates the MIC Editor program, is a compiler for the MIC language. The result of the compiler is the code to be executed by the MICTREE binary decision machine. After compiling the design, the programmer can proceed to simulate the design with MIC Sim (Sub-Section 3.5.3) or to download the genome microprogram into a real MICTREE network.

\subsubsection{Visual BDD}

Visual BDD [65] is a graphical environment used to create flowcharts describing MICTREE genome microprograms. Visual BDD is composed mainly of a binary decision diagram (BDD) editor, a flowchart components editor, and a MIC code generator.

This program presents a very easy-to-use and powerful graphical design environment, allowing the user to deal with complex BDD designs. One of the most interesting features of Visual BDD is the possibility of having hierarchical design views, i.e., to describe the genome microprogram at different levels. One example is presented in Figure $3.23 \mathrm{c}$, where the higher-level view is the flowchart (at the right), and the lower-level views are on the left. This functionality allows an easier and faster development of MICTREE genome microprograms. 


\subsubsection{MIC Sim}

MIC Sim is a MICTREE network simulator [27], which allows us to study the behavior of our artificial organism (the MICTREE network) before the actual implementation. The purpose of the simulator is to relieve the MICTREE user of all the problems involved in the use and management of the real MICTREE network. The user will be able to devote his attention to the development rather than to the physical implementation.

With MIC Sim, the user can easily describe the MICTREE network topology to be simulated and send the genome microprogram (generated by the MIC Compiler) to configure the network and start the simulation.

\subsubsection{Visual MICTREE design environment}

In order to achieve versatility and user-friendliness, we decided to integrate all the MICTREE's software tools in a single graphical design environment code-named Visual MICTREE. This design environment consists of the MIC Editor program, the MIC Compiler, the Visual BDD program, the MICTREE simulator, and a serial loader used to configure the MICTREE network through the computer serial port.

The microcode executed by the MICTREE binary decision machine can be generated by compiling the MIC code written with the text editor, or alternatively (and more simply) by compiling the MIC code generated by Visual BDD. This microcode can then be sent to the MICTREE network through the computer's serial port by using the loader, starting the "life" of our artificial organism.

\subsection{Reliability analysis of the Embryonics architecture}

This section presents briefly the results of the study of the reliability of Embryonics architectures realized by Tyrrell and Ortega at York University [73-75].

To analyse the reliability of MICTREE organisms, the following notation has been used. There are $h \times g$ cells in an organism, from which only a sub-array of $h \times f$ cells will perform the desired function. The remaining $g-f$ columns are spares that replace faulty columns when a fault is detected in a cell. The user defines the number of spare columns that are inserted in an organism to provide a higher level of fault tolerance. Figure 3.25 shows the structure of a MICTREE organism.

In the following analysis, $\lambda$ is the failure rate of a single cell. $\lambda$ is assumed constant and usually expressed as failures per unit time, for example failures per hour or failures per $10^{6}$ hours. The failure rate is determined by the cell's model. The cell's model is a function of parameters that describe its physical implementation, operating characteristics, and the environment in which the device operates [11]. 


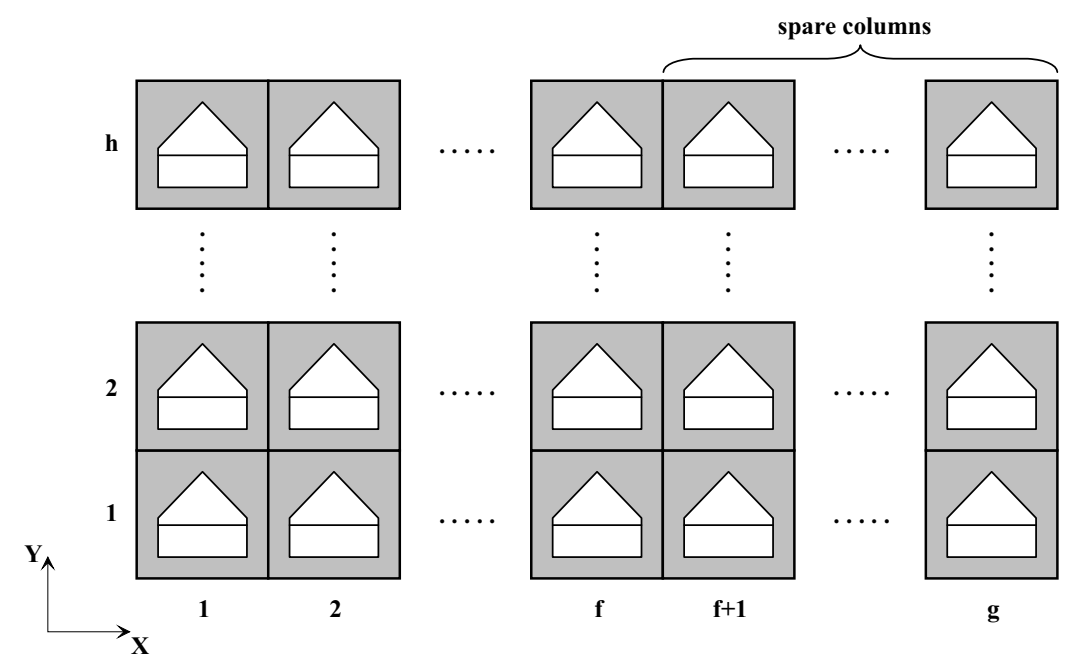

Figure 3.25: Structure of a MICTREE organism

As mentioned in Sub-Section 3.2.1, when a fault occurs in one of the cells within the organism, the corresponding column is logically eliminated by making it transparent to the calculation of coordinates. Coordinates are shifted to the right until a spare column is reached. At that point, cells will perform a new function according to their new set of coordinates. An organism will "live" as long as there are spare columns that can replace transparent ones. When spare columns run out and a new fault arises, the organism "dies".

The reliability of one row of cells can be expressed using the $f$-out-of- $g$ reliability model. In general, an $f$-out-of- $g$ system will remain functional as long as $f$ out of its $g$ components remain functional. The reliability of an $f$-out-of- $g$ system with identical elements is given by the following expression [66]:

$$
R_{f-o u t-o f-g}(t)=\sum_{i=f}^{g}\left(\begin{array}{l}
g \\
i
\end{array}\right) e^{-i \lambda t}\left(1-e^{-\lambda t}\right)^{g-i}
$$

where the reliability of one cell is assumed to follow the exponential law given by:

$$
R(t)=e^{-\lambda t}
$$

Expression 3.26 yields the reliability of one row in the array. The organism's reliability can be expressed as the series connection of $h$ rows. A system with $h$ elements connected in series requires that all its elements be working correctly in order to perform its function. The reliability of a series system with independent elements is given by the multiplication of the reliability expressions of all its elements [66]. Therefore, the reliability of one organism with $h$ rows would be given by: 


$$
R_{\text {org }}(t)=\left(\sum_{i=f}^{g}\left(\begin{array}{l}
g \\
i
\end{array}\right) e^{-i \lambda t}\left(1-e^{-\lambda t}\right)^{g-i}\right)^{h}
$$

If the number of cells in the array allows self-replication of the original organism, then every new organism will perform the same function. Therefore, a correct output will be available as long as there is one surviving organism. Such a structure is modelled by the reliability expression 3.29 for $x$ identical elements connected in parallel.

$$
R_{\text {total }}=1-\left(1-R_{\text {org }}(t)\right)^{x}
$$

where $x$ is the number of organisms in the system.

Figure 3.26a shows the graphic representation of equation 3.28 for organisms with different numbers of spare columns. Figure $3.26 \mathrm{~b}$ shows the corresponding representation of equation 3.29. The following conditions have been assumed:

$\Rightarrow$ the organism's size $h \times g$ is $50 \times 50$ cells;

$\Rightarrow$ the number $x$ of organisms in the system is 6 ; the topology (i.e. $3 \times 2,6 \times 1$, etc.) is not relevant;

$\Rightarrow$ the failure rate $\lambda$ is 1 failure every $10^{6}$ hours;

$\Rightarrow$ cells fail independently from each other.

For the purpose of comparison, the reliability of a $50 \times 50$ cells array with no spare columns is also shown. The benefits of adding redundancy are evident.
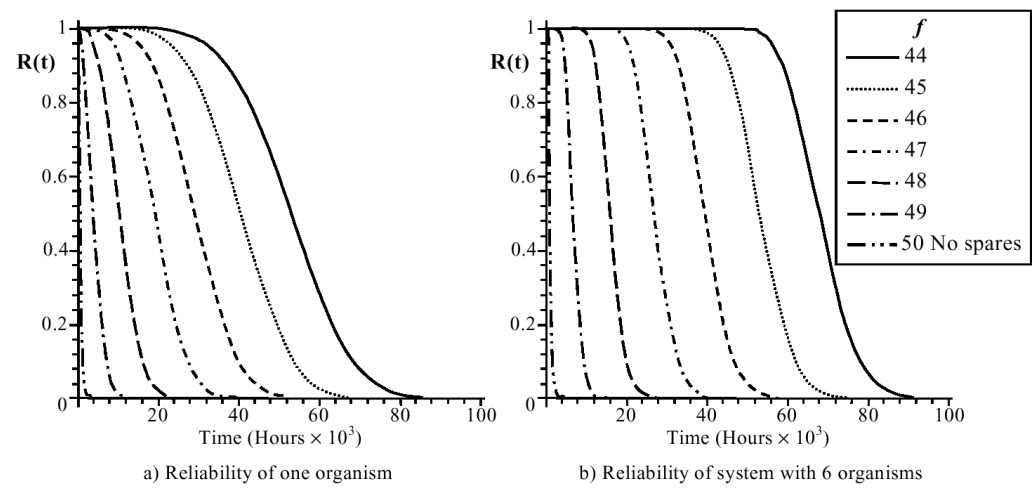

Figure 3.26: Reliability of a $50 \times 50$ MICTREE cells organism with different number of spare columns 
A one-to-one comparison of the graphs in Figures 3.26a and 3.26b shows the effects of self-replication. The more organisms there are, the better the reliability of the system. Figure 3.26 clearly shows that the larger the number of spare columns in the organism, the longer the organism will function correctly. It also shows that the relative improvement in reliability with respect to the number of spare cells increases as the number of spare cells increases. For example, the improvement in reliability when passing from 5 to 6 spare columns is bigger than the improvement when passing from 2 to 3 spare columns.

Figures $3.27 \mathrm{a}$ and $3.27 \mathrm{~b}$ show the graphs of a system with the following characteristics:

$\Rightarrow$ the organism's size $h \times g$ is $50 \times 50$ cells;

$\Rightarrow$ the number $x$ of organisms in the system is 6 ;

$\Rightarrow$ the number $f$ of active cells per row is 44 , i.e. 6 spares per row

$\Rightarrow$ the failure rates $\lambda$ varies;

$\Rightarrow$ cells fail independently from each other.
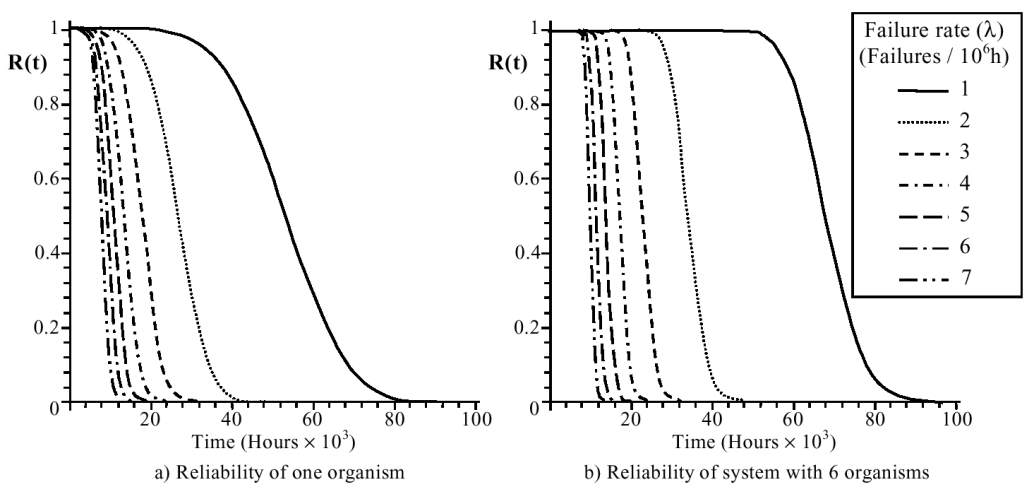

Figure 3.27: Reliability of a MICTREE system for different failure rates

Figure 3.27 shows the high reliability associated to small values of $\lambda$. Decreasing the value of $\lambda$ requires an improvement in the quality of the system's components and, in the majority of cases, the cost associated with such an improvement. However, in highlyreliable systems like satellites or specialized control equipment, the cost of decreasing the value of $\lambda$ is amply justified.

Graphs in Figure 3.28 show the behavior of system reliability for organisms with different number of rows. All the systems share the following characteristics:

$\Rightarrow$ the organism's width $g$ is 50 cells; 
$\Rightarrow$ the organism's depth $h$ is variable ;

$\Rightarrow$ the number $f$ of active cells per row is 44 , i.e. 6 spares per row;

$\Rightarrow$ the failure rate $\lambda$ of cells is 1 failure every $10^{6}$ hours;

$\Leftrightarrow$ cells fail independently from each other.

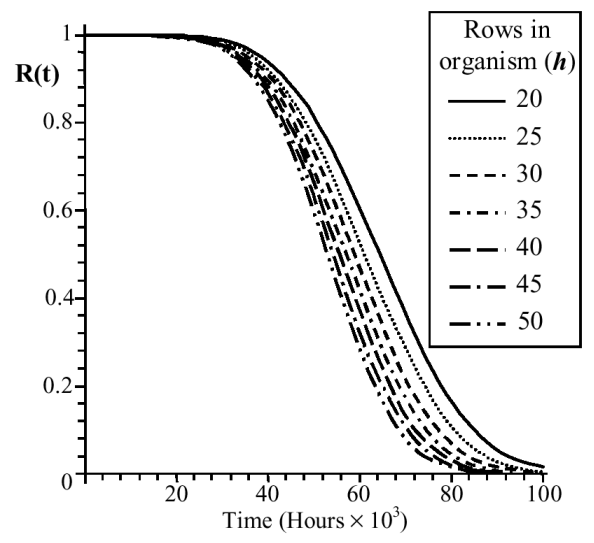

Figure 3.28: Reliability of organisms with different number of rows

Figure 3.28 shows that the reliability of organisms decreases as the number of rows increases. This result is expected from any system whose elements are connected in series. Therefore, when an application is being mapped to a MICTREE array, the number of rows per organism should be minimized. The ideal case would be an organism with only one row, with its reliability given by expression 3.26.

Figure 3.29 shows reliability graphs of seven embryonic systems with different number of MICTREE organisms. The arrangement of organisms is irrelevant; as long as one organism survives, the system will deliver its function. The common characteristics of the systems are:

$\Rightarrow$ the organism's size $g \times h$ is $50 \times 50$ cells;

$\Rightarrow$ the number $f$ of active cells per row is 44 , i.e. 6 spares per row;

$\Rightarrow$ the failure rate $\lambda$ of cells is 1 failure every $10^{6}$ hours;

$\Rightarrow$ cells fail independently from each other.

Figure 3.29 shows that, as the number of organisms increases, the reliability curve becomes steeper, i.e., reliability improves. However, Figure 3.29 also demonstrates that the contribution from new organisms to system reliability decreases as the total number of organisms increases. For example, reliability improves more when passing from two to three organisms than when passing from six to seven. Hence, it is important to consider a cost/benefit analysis before deciding the number of new organisms allocated in a system. 


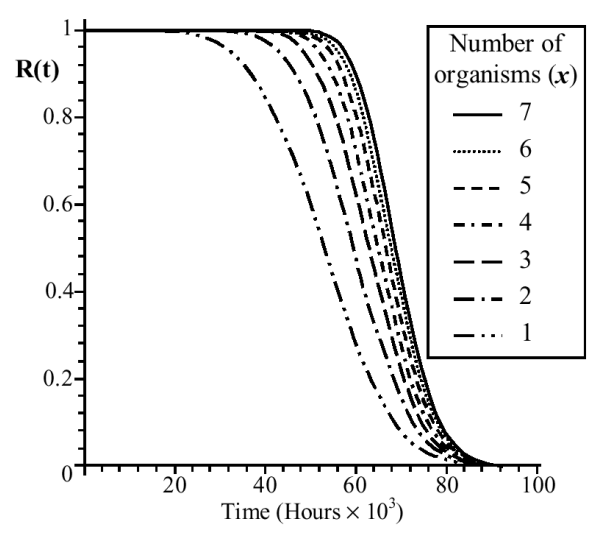

Figure 3.29: Reliability of embryonic systems with different number of MICTREE organisms

\subsection{Conclusion}

The main result of the research is the development of a new kind of coarse-grained FPGA called MICTREE, which is based on a binary decision machine capable of executing a microprogram of up to 1024 instructions. The original features of this FPGA are essentially:

$\Rightarrow$ a completely homogeneous organization of the cellular array;

$\Rightarrow$ an integration of the routing into each cell, both for the short- and the long-distance (bus) connections;

$\Rightarrow$ a sequential execution of microprograms methodically derived from a chosen representation, the binary decision diagram.

The FPGA satisfies the general hypothesis, as well as the three features of the Embryonics project: multicellular organization, cellular differentiation, and cellular division. The MICTREE cell, itself realized with a commercial FPGA and a RAM, was finally embedded into a plastic demonstration module called BIODULE 601, and we showed that an array of BIODULES 601 is capable of self-repair and self-replication.

The artificial organisms exploit hardware redundancy to achieve fault tolerance. The distributed automatic reconfigurability characteristic of the Embryonics approach offers considerable advantages over other reconfiguration strategies where, in most cases, a centralized agent, e.g. the operating system or a central processor, must solve the routing problem.

Diagnosis and reconfiguration functions are performed at the cellular level in the final Embryonics architecture, where each cell is itself decomposed into molecules, each 
molecule embedding built-in self-test $[52,57,102]$. No centralized agent exists. Spare elements are incorporated at different levels of an embryonic system in order to achieve resilience to faults in its constituent molecules and organisms. The reliability model presented in Section 3.6 allows the analysis of the MICTREE architecture for different combinations of spare cells and organisms.

It has been verified that adding spare cells to a system will improve its reliability. A natural strategy to improve system's reliability would be to incorporate as many spares as possible; nevertheless, in some cases, the cost of spares can be very high. Therefore a tool that allows the quantitative comparison of different alternatives is needed. The reliability analysis presented in this chapter can auxiliate this decision-making process. 



\section{Chapter 4}

\section{Specialized Turing Machines}

In the 1930's, before the advent of digital computers, several logicians (Kurt Gödel, Alonzo Church, Stephen Kleene, Emil Post, and Alan Mathison Turing) began to think about the theoretical limits of computation. Alonzo Church and Alan Turing independently arrived, through different approaches, at equivalent conclusions. Both solutions described computability, but while Church (1932-34) described it with $\lambda$-calculus, Turing's idea (1936) was based on a mathematical model of a machine that could compute any computable functions: the Turing machine [34,107].

Turing machines were first described by Alan Turing in his historic paper, "On Computable Numbers, with an Application to the Entscheidungsproblem" [109], which was his answer to the Entscheidungsproblem posed by the German mathematician David Hilbert. Hilbert asked if there existed, in principle, any definite method which could be applied to determine the truth of any mathematical assertion [28].

In this chapter, we will present a brief introduction to Turing machines (Section 4.1) and the details of the implementation of a self-replicating and self-repairing specialized Turing machine, a parenthesis checker, based on a multicellular array of cells (Section 4.2 to Section 4.4).

\subsection{Specialized Turing machines}

In his 1936 paper [109], A. M. Turing defined the class of abstract machines that now bear his name: Turing machines. A specialized Turing machine (Figure 4.1), or simply a Turing machine, is a finite-state machine (the program) controlling a mobile head, which operates on a tape. The tape, composed of a sequence of squares, contains a string of symbols (the data). The head is situated, at any given moment, on some square of the tape and has to carry out three operations to complete a step of the computation (one 


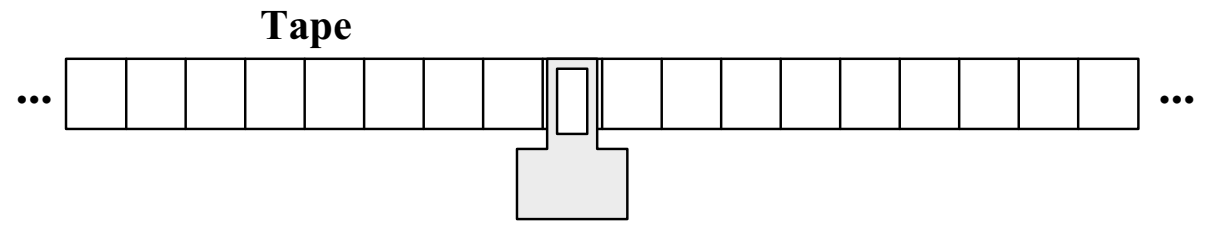

Mobile head

Figure 4.1: A specialized Turing machine.

operation cycle of the finite-state machine). These operations are:

1. reading the square of the tape being scanned;

2. writing on the scanned square;

3. moving the head to an adjacent square (which becomes the scanned square in the next operation cycle).

A Turing machine can be described by three functions $f_{1}, f_{2}, f_{3}$ :

$$
\begin{aligned}
& Q+=f_{1}(Q, S) \\
& S+=f_{2}(Q, S) \\
& D+=f_{3}(Q, S)
\end{aligned}
$$

where $Q$ and $S$ are, respectively, the current internal state (of the finite state machine) and the current input symbol (the symbol on the square of the tape being scanned), and where $Q+, S+$, and $D+$ are, respectively, the next internal state, the next input symbol, and the direction of the head's next move [64].

The tape can be considered as infinite in both directions. However, we will make the restriction that, when the machine starts operating, the tape must be blank, except for some finite number of squares. With this restriction, we can think of the tape as finite at any particular time but capable of being infinitely extended whenever the machine comes to an end of the finite portion.

When a symbol is printed on the tape, the symbol previously there is erased. Of course, it can be preserved if we print the same symbol that was read. Because the head can move either way along the tape, it is possible for it to return to a previously printed location to recover the information inscribed there. This ability provides the machine a sort of rudimentary memory in a sense that the machine can look up the previous symbols and change them if necessary. Since the tape is as long as desired, this memory is potentially infinite. 
At any given time, the read/write mobile head of the Turing machine is positioned on some square on the tape. Furthermore, at any given time, the Turing machine is in one of a finite number of internal states.

A set of quintuples can be used to specify what the machine will do for each possible combination of symbol and state. These quintuples have the following form

(current state, current symbol, next state, next symbol, direction of motion)

or, equivalentely:

$(Q, S, Q+, S+, D+)$

where the third, fourth, and fifth symbols are determined by the first and second according to the three functions $f_{1}$ (4.1), $f_{2}$ (4.2), $f_{3}$ (4.3) mentioned above.

These quintuples indicate that if a Turing machine is now in the current internal state $Q$, and the current input symbol is $S$, the machine will change its current internal state to the next internal state $Q_{+}$, replace the current input symbol on the tape by the next input symbol $S+$, and move the read/write head one square in the given direction $D+$. If a Turing machine is in a condition for which it has no instruction, it halts.

The information contained in the set of quintuples is often represented in the form of a state table, defining the behavior of the machine for each possible combination of symbol and state.

Different formalizations of the notion of a Turing machine define them in slightly different ways. The main difference is in how much a machine can do in a single step: does the machine have to choose between printing a symbol on the tape and moving along the tape, or can it do both in a single step? The former is called the quadruple approach, the latter the quintuple approach. In fact there is no theoretical difference between the two approaches [6].

\subsection{A parenthesis checker}

According to Minsky [64] (pp. 121-123), the problem is to decide whether a sequence of left (open) and right (closed) parentheses is well-formed. That is, whether they can be paired off from inside to outside so that each left parenthesis has a right-hand mate. A good procedure for checking parentheses consists of searching to the right for a right (closed) parenthesis, then searching to the left for its mate (open), and removing both. This procedure is repeated until no more pairs are found. If any unmatched symbols remain, the expression is not well-formed, and conversely.

For checking the expression "(()", for example, we prepare the tape as shown in Figure 4.2a, where the beginning and the end of the sequence to be checked are marked by " $A$ " symbols. Figure $4.2 \mathrm{~b}$ shows the state table of the parenthesis-checking finite-state 
machine.

The read/write head starts by reading the first left parenthesis (Figure 4.2a). The initial state of the finite state-machine (Figure 4.2b) is the " $\rightarrow$ " state. For each couple $H, T$ (head state, tape state) ${ }^{1}$, the state table of Figure $4.2 \mathrm{~b}$ produces a new couple $\mathrm{H}+, \mathrm{T}+$ giving the next head state $H+$, belonging to the set $\{\rightarrow, 0 \leftarrow, 1 \leftarrow, \uparrow\}$ and the next tape state $T+$, belonging to the set $\{X,(, A), 0,1$,$\} .$

In this particular case, we combined the internal state $H$ with the direction of the tape $D$. For $H=\{\rightarrow\}$ the head will move to the right, for $H=\{0 \leftarrow, 1 \leftarrow\}$ the head will move to the left, and for $H=\{\uparrow\}$ the head will stop.

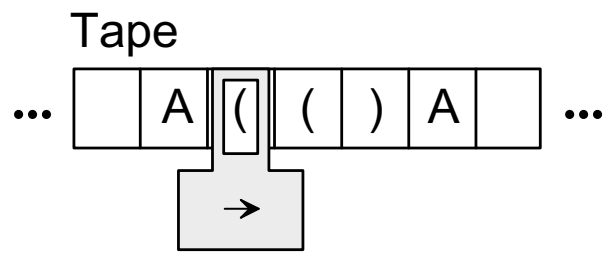

Mobile head

(a)

\begin{tabular}{|c|c|c|c|c|c|c|}
\hline $\mathrm{H}+, \mathrm{T}_{+}$ & $\begin{array}{l}T \\
X\end{array}$ & ( & $A$ & ) & 0 & 1 \\
\hline$->$ & $->, X$ & $->,($ & $1<-, A$ & $0<-, X$ &,-- &,-- \\
\hline $0<-$ & $0<-, X$ & $->, X$ & $\hat{\imath}, 0$ & $0<-$, ) &,-- &,-- \\
\hline $1<-$ & $1<-, X$ & $\hat{\imath}, 0$ & $\hat{\imath}, 1$ &,-- &,-- &,-- \\
\hline$\hat{\imath}$ &,-- &,-- &,-- &,,-- & $\hat{\imath}, 0$ & $\hat{\imath}, 1$ \\
\hline $\mathrm{H}$ & & & & & & \\
\hline
\end{tabular}

(b)

Figure 4.2: Parenthesis checker. (a) Tape with expression to check. (b) State table.

Figure 4.3 traces out the operation of our parenthesis-checking Turing machine on the "(()" expression. We see that one pair has been removed and replaced by the symbols " $X$ ". As the expression " $(()$ " is not well-formed, the last symbol written on the tape by the head is " 0 ". At this point the finite-state machine has reached the "个" state and stops.

\footnotetext{
${ }^{1}$ The head and tape states correspond, respectively, to the current internal state $Q$ and the current input symbol $S$ defined in Section 4.1
} 


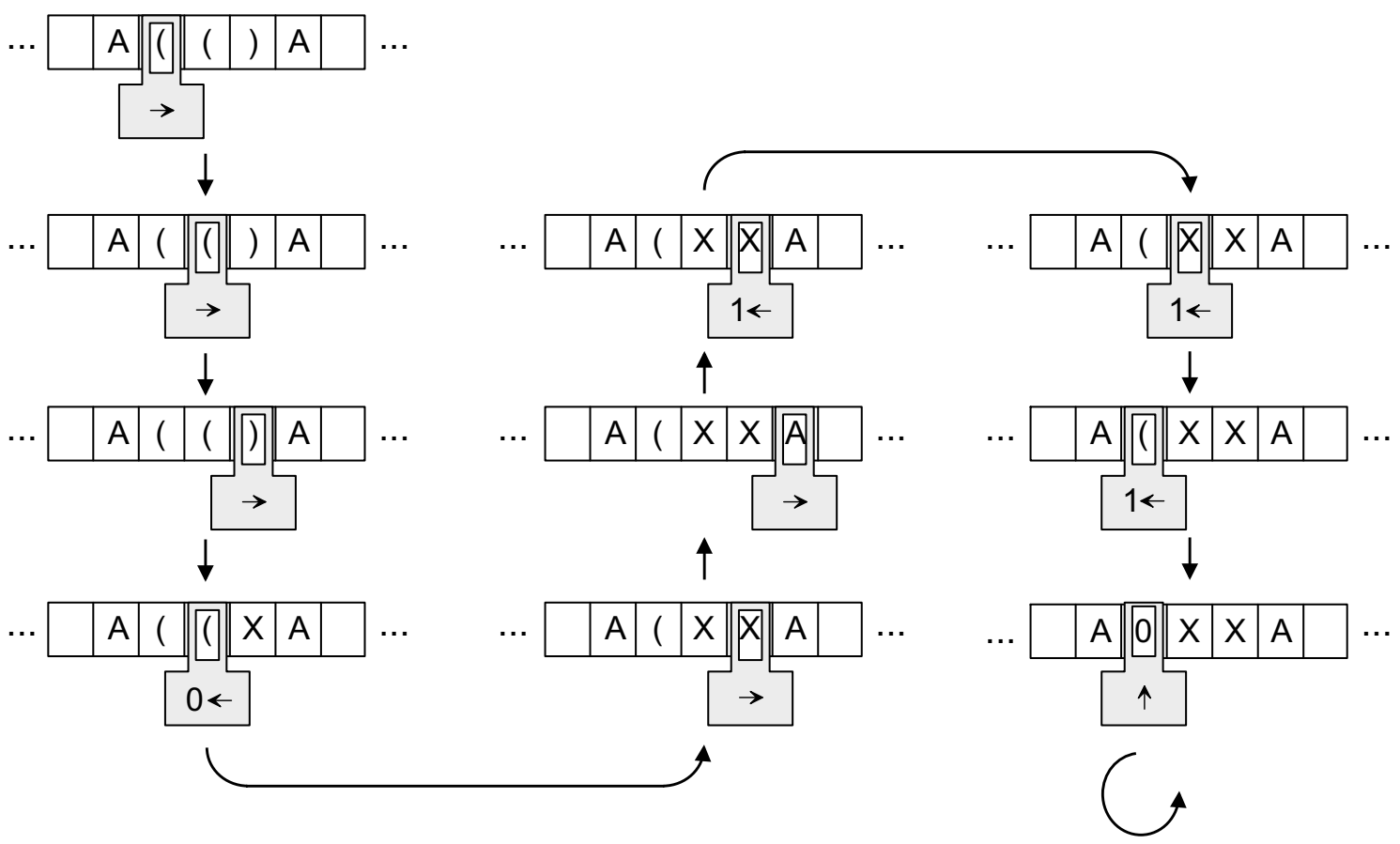

Figure 4.3: Step-by-step operation operation of the parenthesis-checking Turing machine for the "(()" expression.

\subsection{Self-replication of specialized Turing machines on a multicellular array: the Embryonics approach}

\subsubsection{Embryonics features}

Essentially, Embryonics is a modified automata-based approach in which three biologically inspired principles are employed: multicellular organization, cellular differentiation, and cellular division. According to the multicellular organization feature, the artificial organism is divided into a finite number of cells (Figure 4.4), where each cell realizes a unique function, described by a sub-program called the gene of the cell.

A multicellular realization (Figure 4.4) of the parenthesis checker example can be implemented as a multicellular organism with the following structure:

$\Rightarrow$ a mobile head described by a row of cells $(Y=2)$, where only one cell is in an active state, while the others are in a passive one. The cell in the active state is used to indicate the mobile head position and which way it will move;

$\Rightarrow$ a tape described by a row of cells $(Y=1)$ and implementing the five squares of the Turing machine tape containing the sequence " $\mathrm{A}(() \mathrm{A}$ ". 


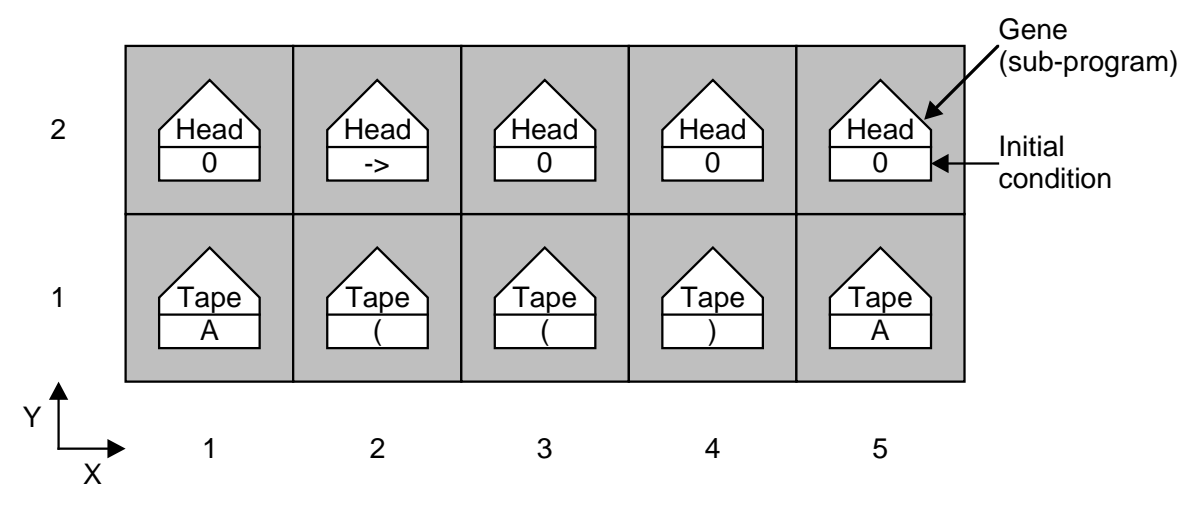

Figure 4.4: Multicellular organization of a specialized Turing machine, a parenthesis checker.

Our specialized Turing machine, a parenthesis checker, is thus implemented with ten cells and features two distinct genes, the tape gene and the head gene (Figure 4.4). Each cell is associated with some initial condition. In our example, the head cells are distinguished by the initial values " 0 " and " $\rightarrow$ ", the tape cells by "A", "(", and ")" values.

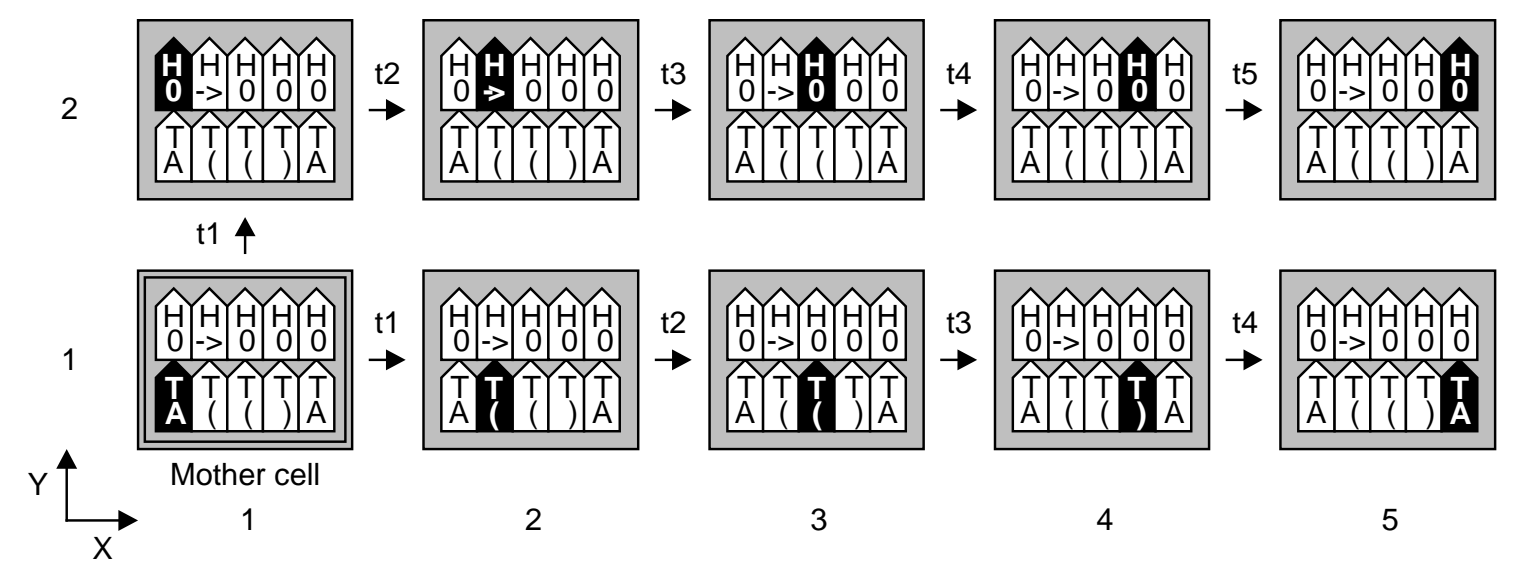

Figure 4.5: Cellular differentiation and cellular division of a specialized Turing machine, the parenthesis checker; $t_{1} \ldots t_{5}$ : five successive divisions.

Let us call genome the set of all the genes of an artificial organism, where each gene is a sub-program characterized by a set of instructions, by an initial condition, and by a position (the coordinates $X, Y$ of the cell). Figure 4.4 then shows the genome of our Turing machine, with the corresponding horizontal $(X)$ and vertical $(Y)$ coordinates. Let then each cell contain the entire genome (Figure 4.5): depending on its position in the array, i.e., its place in the organism, each cell can interpret the genome and extract and execute the gene (with its initial condition) which configures it. According to the cellular differentiation feature, it can interpret any gene of the genome (including the initial condition), given the proper coordinates. 
At startup, the mother cell or zygote (Figure 4.5), arbitrarily defined as having the coordinates $X, Y=1,1$, holds the one and only copy of the genome. At time $t_{1}$, according to the cellular division feature, the genome of the mother cell is copied into the two neighboring (daughter) cells to the north and to the east. The process then continues until the two-dimensional space is completely programmed. In our example, the furthest cell is programmed at time $t_{5}$.

In all living beings, the string of characters which makes up the DNA is executed sequentially by a chemical processor, the ribosome. Drawing inspiration from this biological mechanism, we will use a microprogram to compute first the coordinates of the artificial organism, then the initial conditions of each cell, the tape gene and the head gene, and finally the complete genome. The calculation of this microprogram is detailed in $[50,53]$. Its software implementation is written in the MIC programming language, which is interpreted by the MICTREE cell, both described in Chapter 3.

\subsubsection{Self-repair and self-replication}

In order to demonstrate self-repair, we added two spare cells in each row, to the right of the original Turing machine, all identified by the same horizontal coordinate $(X=6$ in Figure 4.6). The spare cells may be used not only for self-repair, but also for the example of a Turing machine necessitating the growth of the tape of arbitrary, but finite, length.

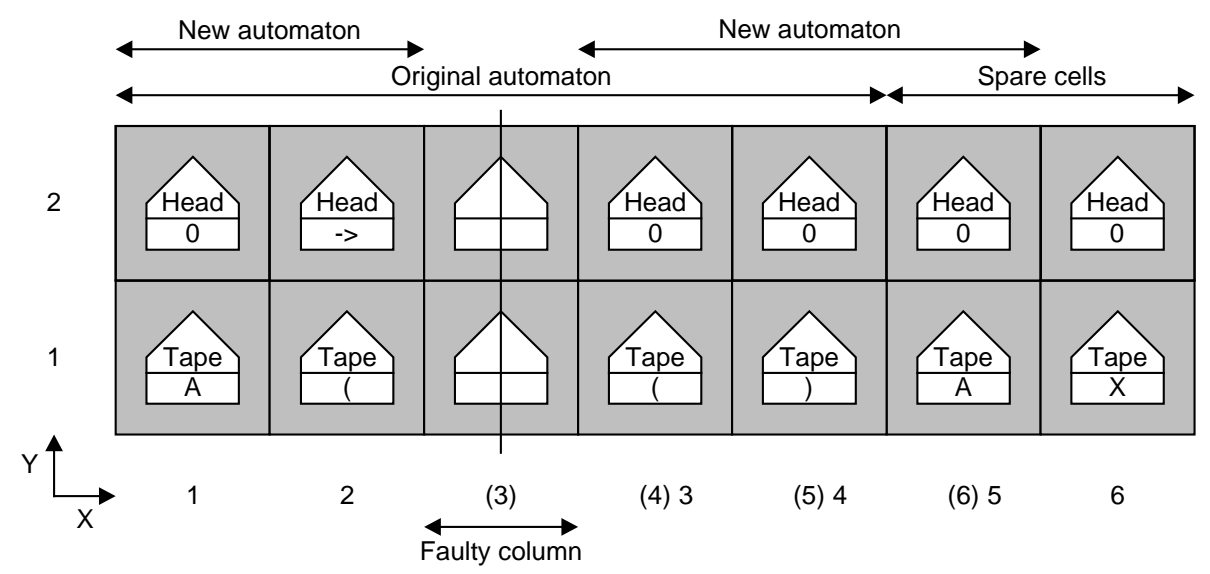

Figure 4.6: Self-repair of a 10-cell parenthesis checker in a 14-cell array.

The existence of a fault is detected by a KILL signal which is calculated in each artificial cell by a built-in self-test realized at the FPGA level. The state $K I L L=1$ identifies the faulty cell and the entire column to which the faulty cell belongs is considered faulty, and is deactivated (column $X=3$ in Figure 4.6). All the functions of the artificial cells to the right of the column $X=2$ are shifted by one column to the right. Obviously, this process requires as many spare columns to the right of the array as there are faulty columns to repair (there are two spare columns in the example of Figure 4.6). It also implies that 


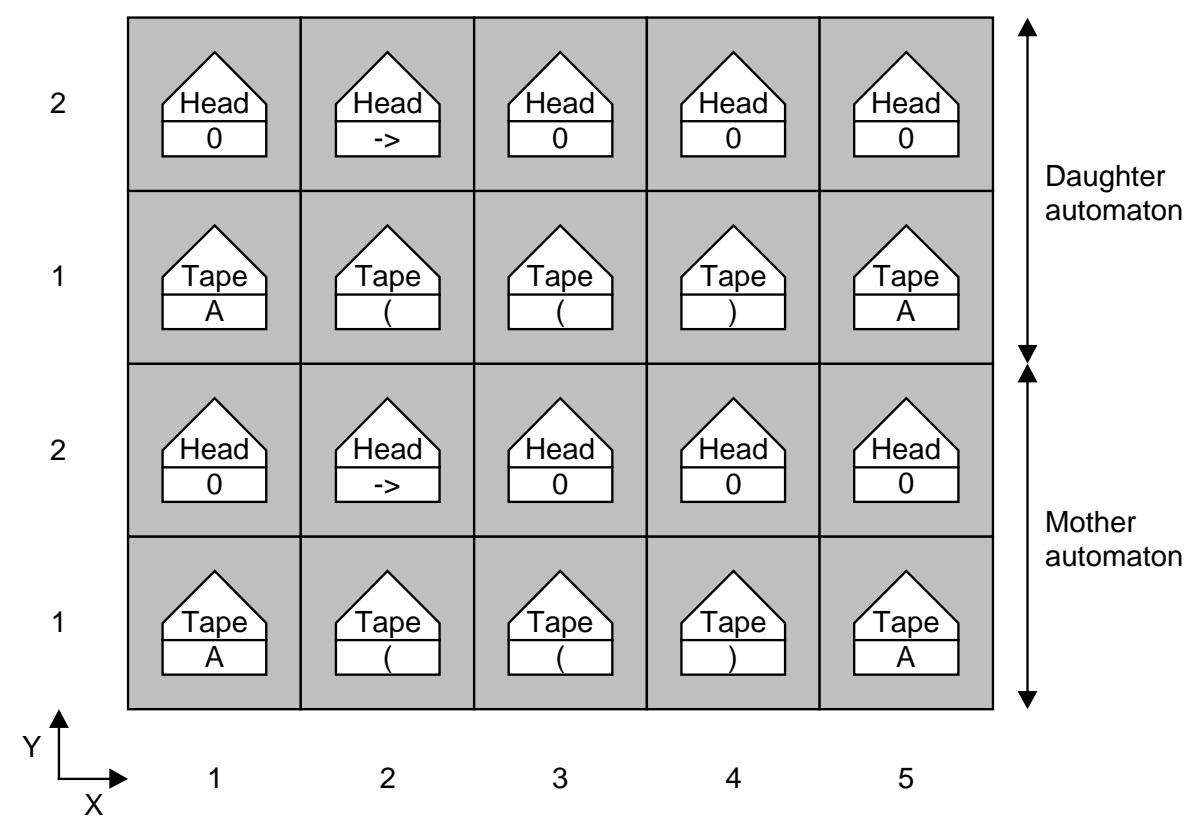

Figure 4.7: Self-replication of a 10-cell parenthesis checker in a 20-cell array.

the artificial cell has the capability of bypassing the faulty column and shifting to the right all or part of the original cellular array. During such a process, the actual values calculated by the cells are destroyed and the whole calculation should be restarted.

The self-replication of an artificial organism rests on two hypotheses:

$\Rightarrow$ there exists a sufficient number of spare cells (unused cells on the upper side of the array, at least ten for our example);

$\Rightarrow$ the calculation of the coordinates produces a cycle at the cellular level ( $Y=1 \rightarrow 2$ $\rightarrow 1 \rightarrow 2$ in Figure 4.7).

As the same pattern of coordinates produces the same pattern of genes (with the initial conditions), self-replication can be easily accomplished if the microprogram of the genome, associated with the homogeneous network of cells, produces several occurrences of the basic pattern of coordinates $(Y=1 \rightarrow 2$ in Figure 4.4). In our example, repetition of the vertical coordinate pattern, i.e., the production of the pattern $Y=1 \rightarrow 2 \rightarrow 1 \rightarrow 2$ (Figure 4.7), produces one copy, the daughter automaton, of the original mother automaton. Given a sufficiently large space, the self-replication process can be repeated for any number of specimens in the $Y$ axis (remember that the $X$ axis is reserved for self-repair and/or for a possible growth of the Turing machine).

With a sufficient number of cells, it is obviously possible to combine self-repair (or growth) toward the $X$ direction and self-replication toward the $Y$ direction. 


\subsection{Detailed calculation of a Turing machine}

\subsubsection{Specifications}

As described in Section 4.2, a parenthesis checker has to decide whether a sequence of left (open) and right (closed) parentheses is well-formed. A good procedure for checking parentheses consists of searching to the right for a right (closed) parenthesis, then searching to the left its mate (open), and removing both. This procedure is repeated until no more pairs are found. If any unmatched symbols remain, the expression is not well-formed, and conversely.

t0 \begin{tabular}{|lll|l|l|l|}
\hline 0 & $\rightarrow$ & 0 & 0 & 0 & H state \\
\cline { 1 - 1 } & $(1$ & ( & ) & A & T state \\
\cline { 1 - 3 } & &
\end{tabular}

$\mathrm{t} 1$

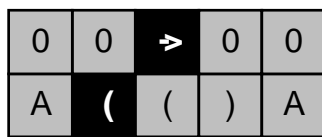

t2

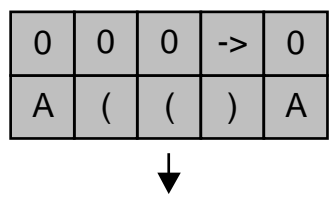

t3

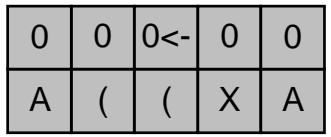

t6

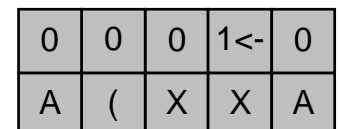

t5

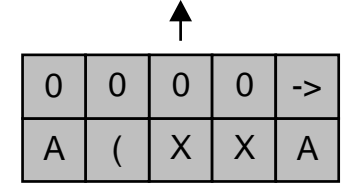

t4

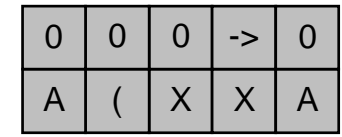

t7

\begin{tabular}{|c|c|c|c|c|}
\hline 0 & 0 & $1<-$ & 0 & 0 \\
\hline$A$ & ( & $X$ & $X$ & $A$ \\
\hline
\end{tabular}

t8

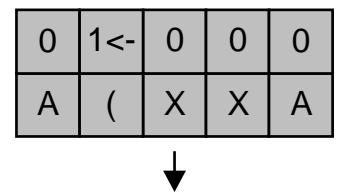

t9

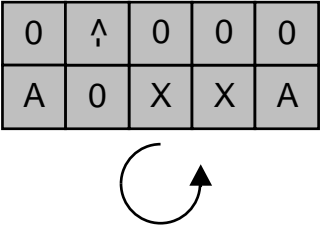

Figure 4.8: Parenthesis checker. Successive H and T states during a 10-step sequence $(t 0 . . . t 9)$.

Thanks to the state table of Figure 4.2 and to the multicellular architecture of Figure 4.4, it is possible to describe the parenthesis checking as follows (Figure 4.8):

$\Rightarrow$ the initial couple $H, T$ (Figure 4.8: time $t 0)$ is defined in our example as $H, T=\rightarrow,($ and produces a next couple $H+, T+=\rightarrow,($. The result of this computation may be illustrated as follows (Figure 4.8: time $t 1$ );

$\Rightarrow$ the next head state $H+$ is " $\rightarrow$ "; the read/write head moves one square to the right and displays this state in the new square;

$\Rightarrow$ the next tape state $T+$ is "("; the square of the tape read by the head at time $t 0$ is unchanged. 
For the moment, we assume that the read/write head can move and has, at a given time, one single position. All the squares with $H=0$ are, therefore, without any physical significance. This process continues until time $t 9$, when no more changes are possible. After going through the head states " $\rightarrow$ " (move to the right), " $0 \leftarrow$ " (move to the left, 1st type), "1 $\leftarrow$ " (move to the left, 2 nd type), the finite-state machine reaches finally the

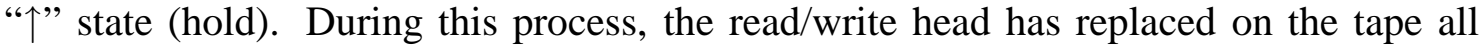
the left "(" and right ")" parentheses by the symbol " $X$ ". As the expression "(()" is not well-formed, the last symbol written on the tape by the head is " 0 ". Conversely, if the expression were well-formed, the last symbol would be " 1 ".

Conventional Turing machines consist of a tape, not infinite, but as long as desired, and a single read/write mobile head controlled by a finite-state machine. In order to obtain a cellular implementation for our parenthesis checker which would be compatible with the homogeneous cellular space defined above, we will realize our Turing machine as follows (Figure 4.9):

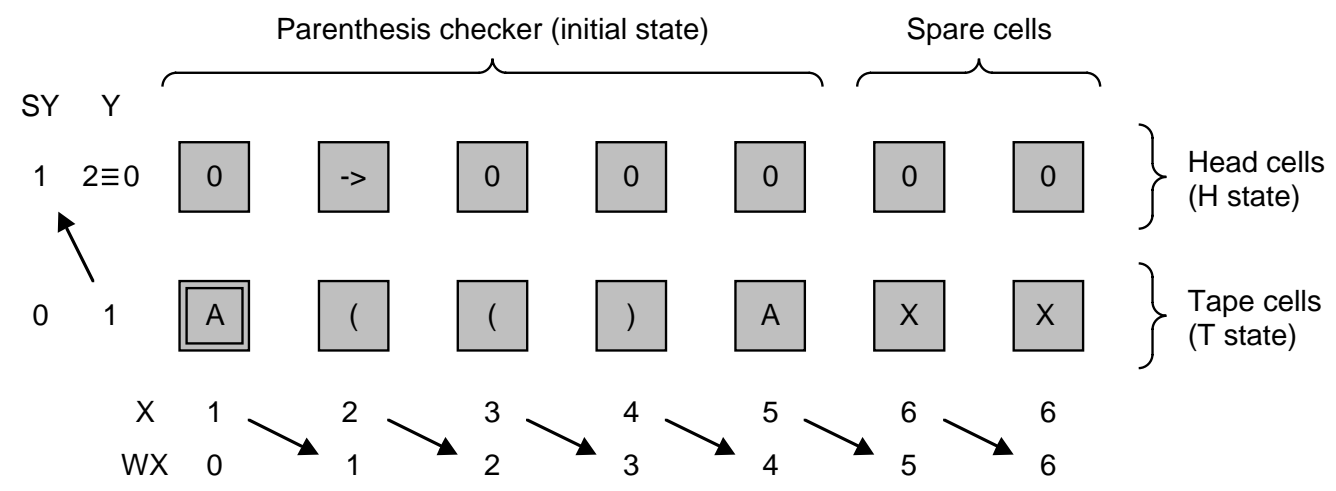

Figure 4.9: A 10-cell $(X=1 \ldots 5)$ parenthesis checker with four spare cells $(X=$ 6); WX: horizontal coordinate of the western neighboring cell; SY: vertical coordinate of the southern neighboring cell.

$\Rightarrow$ The cellular space is divided in two rows, identified by the vertical coordinate $Y$ $(Y=1,2)$, and by $N$ columns, identified by the horizontal coordinate $X(X=1 \ldots N)$. In our example, defined by Figure $4.4, N$ is equal to 5 . In order to demonstrate self-repair, we have decided to add two spare cells in each row, to the right of the Turing machine, all identified by the same horizontal coordinate $N+1(X=6$ in our example); for technical reasons, each cell also contains the coordinates $W X$ of its western neighbor and $S Y$ of its southern neighbor. By definition, the mother cell always has the coordinates $X, Y=1$, 1, i.e. $W X, S Y=0,0$.

$\Rightarrow$ The upper row, identified by $Y=2$, realizes the moving read/write head; all the cells of this row are in a new quiescent head state $(H=0)$, except for one cell which implements the moving head with a non-quiescent state $(H=$ " $\rightarrow$ ", " $0 \longleftarrow$ ", " $1 \leftarrow$ " or “个”). 
$\Rightarrow$ The lower row, identified by $Y=1$, realizes the conventional tape. At the start, all the cells of this row are in one of the three tape states $T=$ " $A$ ", "(" or ")"; the spare cells are in the quiescent tape state $(T=X)$. At the end of the computation process, all the cells in the "(" and ")" states are replaced by " $X$ " state, except for the unique cell giving the final result ( $T=1$ for a well-formed expression, $T=0$ in the contrary).

In conclusion, each cell of the upper row (the head cell) must contain the complete information for calculating the next head state $H+$, while each cell of the lower row (the tape cell) must contain the whole information for calculating the next tape state $T+$. It must also be pointed out that the spare cells, at the right of the cellular space, may be used not only for self-repair, as in our example, but also for other examples of Turing machines necessitating a growth of the tape of any, but not infinite, length.

\subsubsection{Coordinates and initial conditions}

The local horizontal coordinate $(X)$ of a given cell is computed as a function of the horizontal coordinate of its western neighbor $(W X)$. If we represent the coordinate $W X$ in its binary form $W X 2: 0$, the specifications of Figure 4.9 allow us to derive directly the Karnaugh map for $X$ (Figure 4.10a). A "don't care" condition $(\Phi)$ is specified for the unused value $W X 2: 0=111$.

The use of Karnaugh map for simplifying binary decision trees [47,48] generates a tree with seven branches (Figure 4.10b), each represented by a block in the map. For a microprogrammed realization, the binary decision tree of Figure $4.10 \mathrm{~b}$ is the flowchart for the gene of $X$ coordinate. Using test instructions and assignment instructions, the software implementation of this flowchart leads to the sub-program (or gene) Xcoord of Figure 4.10c.

The local vertical coordinate $(Y)$ of a given cell is computed as a function of the vertical coordinate of its southern neighbor $(S Y)$. Unlike the organization of the rows, which requires the placement of spare cells (for self-repair and/or extension of the tape), we wish to design a cycle on the vertical coordinate in order to allow self-replication. Such a cycle is described by the expression $Y=1 \rightarrow 2 \rightarrow 1 \rightarrow 2$ (Figure 4.7), which we code $Y=1 \rightarrow 0 \rightarrow 1 \rightarrow 0$ (Figure 4.9). The trivial Karnaugh map of Figure 4.11a, therefore, defines $Y$ as a function of $S Y O$ (the unique binary variable describing $S Y$ ) and leads to the final binary decision tree and flowchart of Figure 4.11b.

The difficulty of setting a cellular space in an initial configuration is generally underestimated. Starting self-replication with the historical von Neumann's automaton [111] would require that a given state (out of 29) be assigned to approximately 200'000 elements. In our case, computing the initial conditions, i.e. setting each cell of the cellular space in a given head state $H$ or tape state $T$ according to Figure 4.9, is a part of the complete microprogram. In other words, it is a gene of the complete genome. 


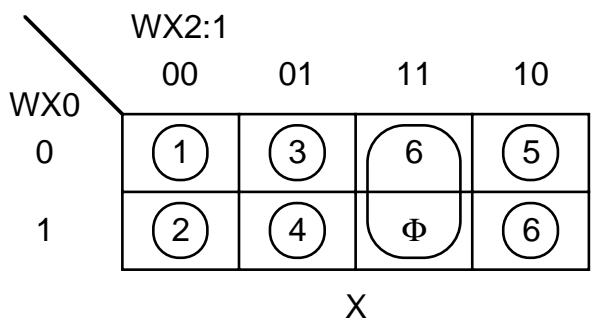

(a)

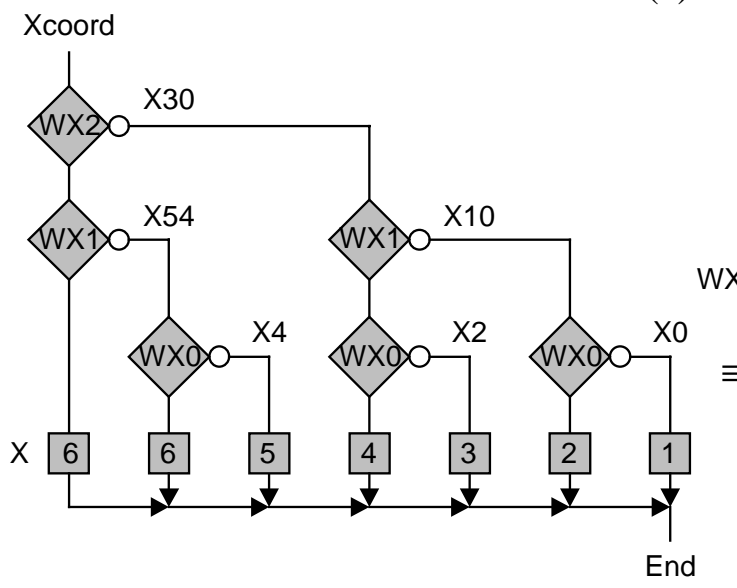

(b)

$\begin{array}{ll}\text { Xcoord: } & \text { if } W X 2 \text { else X30 } \\ & \text { if } W X 1 \text { else X54 } \\ & \text { do } X=6 \\ & \text { goto End } \\ & \text { if } W X 0 \text { else } X 4 \\ & \text { do } X=6 \\ & \text { goto End } \\ & \text { do } X=5 \\ X 4: \quad & \text { goto End } \\ & \text { if } W X 1 \text { else X10 }\end{array}$

$\begin{array}{ll} & \text { if wX0 else X2 } \\ & \text { do } x=4 \\ & \text { goto End } \\ \text { X2: } & \text { do } x=3 \\ & \text { goto End } \\ \text { X10: } & \text { if wXo else X0 } \\ & \text { do } x=2 \\ & \text { goto End } \\ \text { X0: } & \text { do } x=1 \\ \text { End: } & \ldots\end{array}$

(c)

Figure 4.10: Computing the horizontal X coordinate (sub-program Xcoord). (a) Karnaugh map. (b) Binary decision tree and flowcharts. (c) Assembly language sub-program.

The methodology is roughly the same as that used above for computing the coordinates. The initial state of each cell of the cellular space (Figure 4.9) is expressed as a function of the horizontal coordinate of its western neighbor $(W X)$ and of the vertical coordinate of its southern neighbor $(S Y)$. If we represent the coordinate $W X$ in its binary form $W X 2: 0$ and $S Y$ as $S Y 0$, the specifications of Figure 4.9 allow us to derive directly the Karnaugh map for $R E G$ (Figure 4.12a), i.e. the state register of each cell ( $R E G=H$ for head cells, $R E G=T$ for tape cells). A "don't care" or $\Phi$ condition is specified for the unused value $W X 2: 0=111$. The twelve blocks of the Karnaugh map generate a simplified 


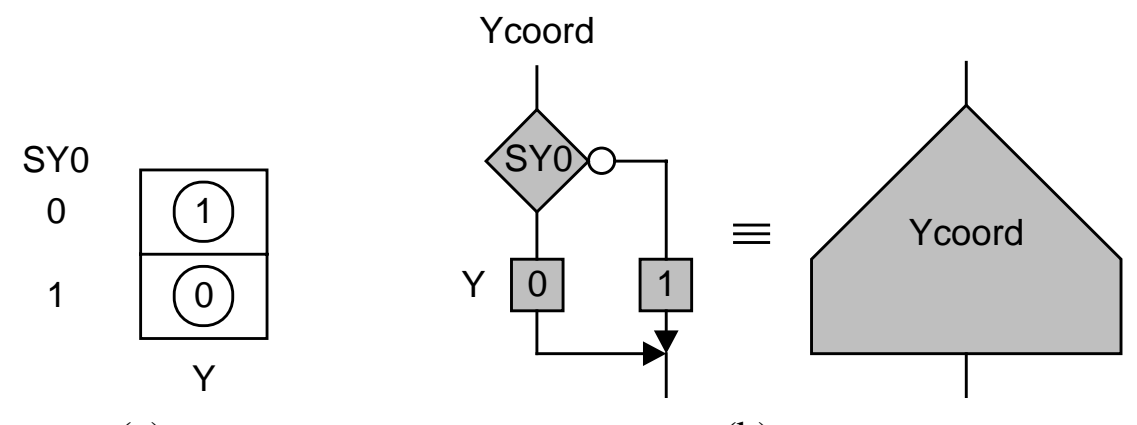

(a)

(b)

Figure 4.11: Computing the vertical $Y$ coordinate (sub-program Ycoord). (a) Karnaugh map. (b) Binary decision tree and flowchart.

binary decision tree with twelve branches, which in turn generates the flowchart of the Initcond sub-program (Figure 4.12b).

\subsubsection{Genes and genome}

Starting from the state table of Figure 4.2b, we wish to express the next tape state $T+$ as a function of the present tape state $T$ and the present head state $H$. Using the Karnaugh map of Figure 4.13a, we first must add the new head state $H=0$, which is the quiescent head state introduced above (Subsection 4.4.1) for the cellular realization of our Turing machine. The new Karnaugh map is then derived from the original state table according to the following additions and tranformations:

$\Rightarrow$ The row for the head state $H=0$ is completed. The examination of the sequence in Figure 4.8 shows that $T+$ is a neutral or no-operation state (NOP) or a "don't care" condition $(\Phi)$.

$\Rightarrow$ Values for $T+$ are borrowed from Figure 4.2b. When $T+=T$, the next tape state $T+$ can be replaced by a NOP state; a dash is replaced by a "don't care" condition $(\Phi)$.

$\Rightarrow$ A binary coding is proposed for both $T$ and $H$ states. This coding was chosen in order to obtain the usual configuration for a Karnaugh map; this map is completely developed for the eight columns (eight $T$ states) and partially for the five rows (in order to obtain simple boolean expressions, we introduce a non-minimal coding and we use only a small subset of the 16 possible $H 3: 0$ states).

We thus obtain a partial 7-variable Karnaugh map, which is convenient enough for our simplification needs. Recalling that the two half-maps (for $T 2=0$ and $T 2=1$ ) are 


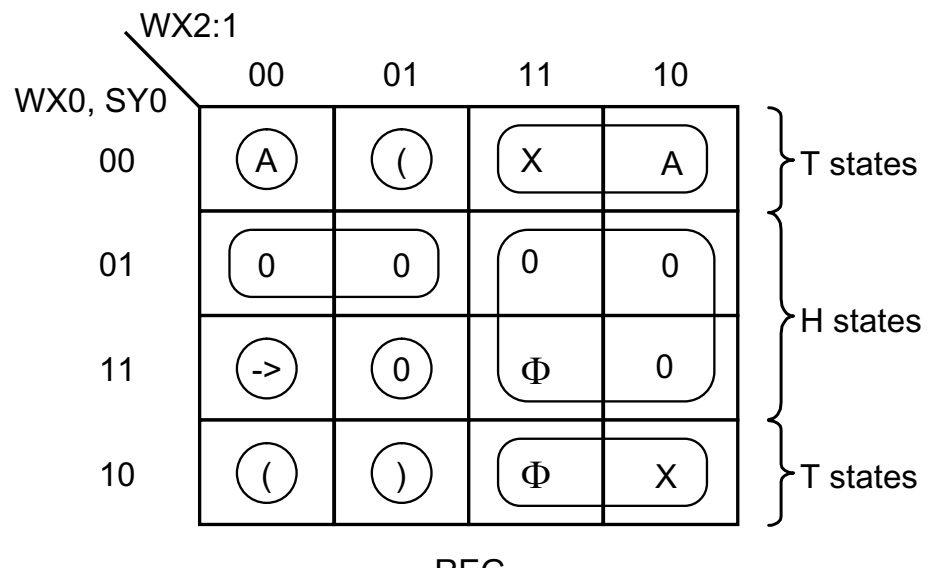

(a)

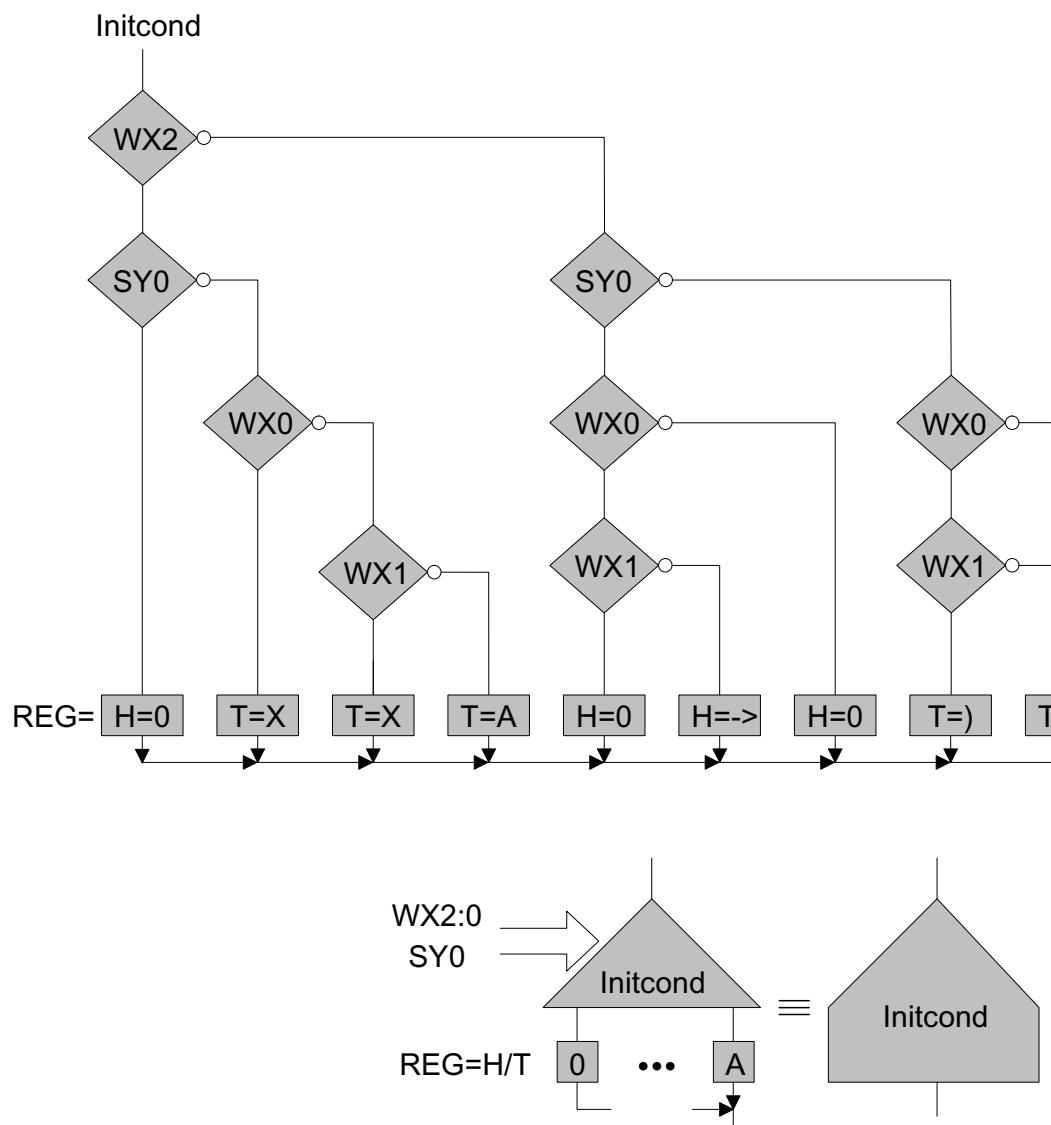

(b)

Figure 4.12: Computing the initial conditions (sub-program Initcond). (a) Karnaugh map. (b) Binary decision tree and flowcharts.

adjacent, we obtain nine blocks in the Karnaugh map, a binary decision tree with nine branches and the corresponding flowchart of the sub-program Tapegene (Figure 4.13b). 


\begin{tabular}{|c|c|c|c|c|c|c|c|c|c|c|}
\hline \multicolumn{2}{|c|}{$\mathrm{T}_{+}$} & $\begin{array}{c}X \\
000\end{array}$ & ( & $\begin{array}{c}A \\
011\end{array}$ & $\begin{array}{c}\text { ) } \\
010\end{array}$ & $\begin{array}{c}0 \\
100\end{array}$ & $\begin{array}{c}- \\
101\end{array}$ & - & $\begin{array}{c}1 \\
110\end{array}$ & $\begin{array}{c}\mathrm{T} \\
\mathrm{T} 2: 0\end{array}$ \\
\hline & 0001 & NOP & NOP & NOP & $X$ & $\Phi$ & $\Phi$ & $\Phi$ & $\Phi$ & \\
\hline $0<-$ & 0100 & NOP & $X$ & 0 & NOP & $\Phi$ & $\Phi$ & $\Phi$ & $\Phi$ & \\
\hline $1<-$ & 1100 & NOP & 0 & 1 & $\Phi$ & $\Phi$ & $\Phi$ & $\Phi$ & $\Phi$ & \\
\hline$\hat{\imath}$ & 0010 & $\Phi$ & $\Phi$ & $\Phi$ & $\Phi$ & NOP & $\Phi$ & $\Phi$ & NOP & \\
\hline 0 & 0000 & NOP & NOP & NOP & NOP & $\Phi$ & $\Phi$ & $\Phi$ & $\Phi$ & \\
\hline & H3:0 & & & & & & & & & \\
\hline
\end{tabular}

(a)

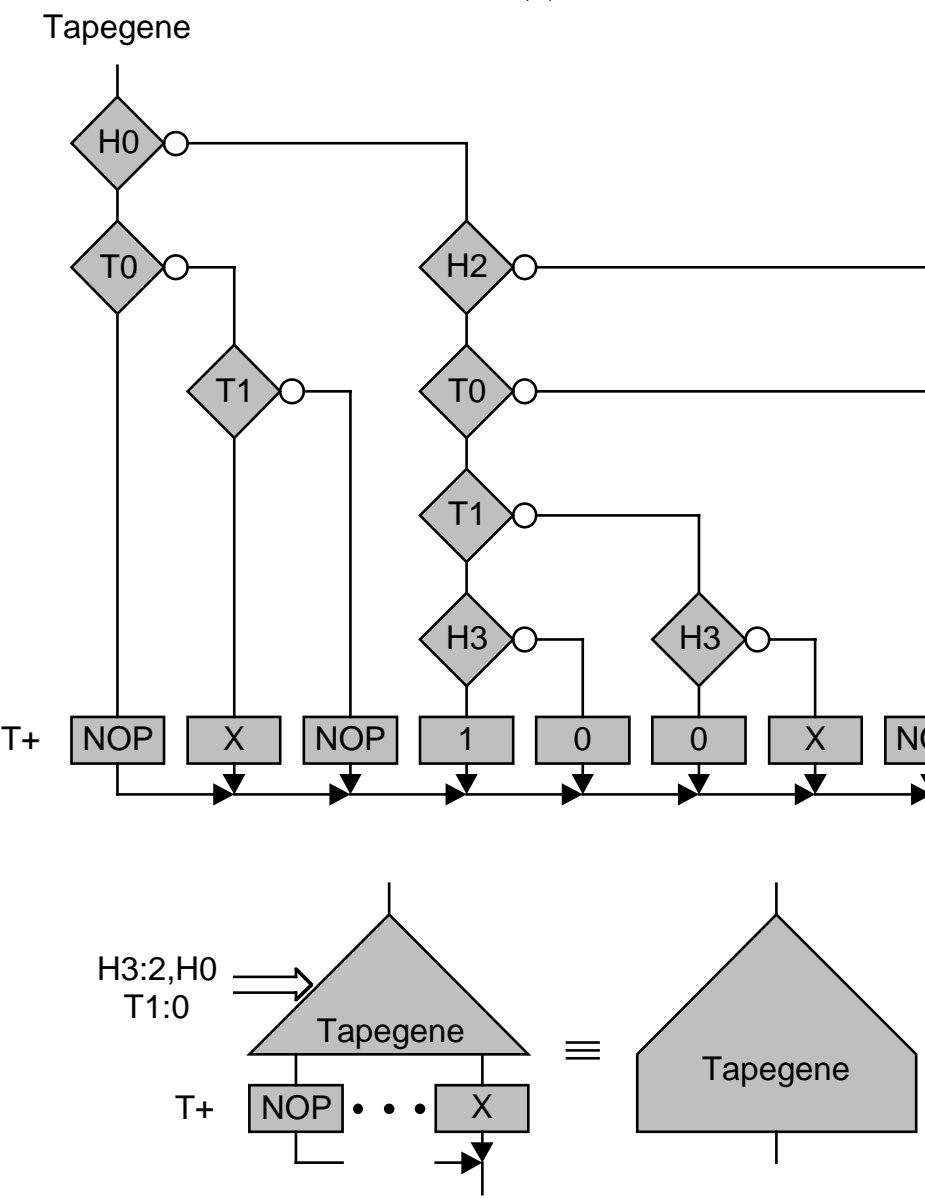

(b)

Figure 4.13: Tape gene computation (sub-program Tapegene). (a) Karnaugh map. (b) Binary decision tree and flowcharts.

As illustrated in Figure 4.8, we have to keep in mind that the head moves to the right if the next head state $H+$ is equal to " $\rightarrow$ ", and to the left if $H+$ is equal to " $0 \longleftarrow$ " or " $1 \leftarrow$ ". We therefore have to consider three different situations which will be combined to obtain the final microprogram calculating the next head state $H+$ : 


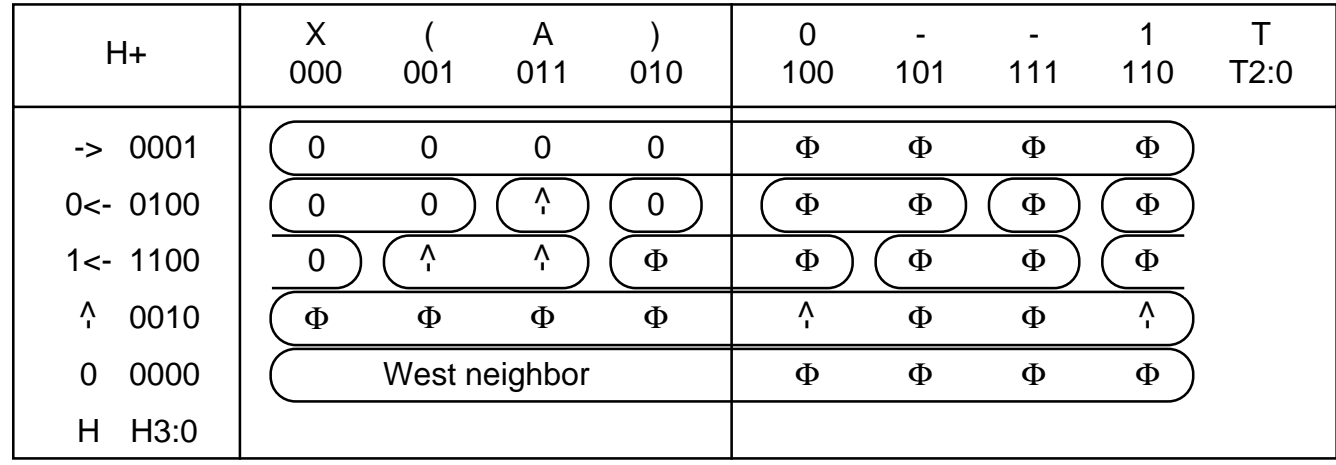

Central cell

(a)

\begin{tabular}{|c|c|c|c|c|c|c|c|c|c|}
\hline $\mathrm{H}+$ & $\begin{array}{c}X \\
000\end{array}$ & 001 & $\begin{array}{c}A \\
011\end{array}$ & $\begin{array}{c}) \\
010\end{array}$ & $\begin{array}{c}0 \\
100\end{array}$ & $\begin{array}{c}- \\
101\end{array}$ & - & $\begin{array}{c}1 \\
110\end{array}$ & $\begin{array}{c}\text { WT } \\
\text { WT2:0 }\end{array}$ \\
\hline$->0001$ & $->$ & $->$ & 0 & 0 & $\Phi$ & $\Phi$ & $\Phi$ & $\Phi$ & \multirow{6}{*}{010 test } \\
\hline $0<-0100$ & 0 & $->$ & 0 & 0 & $\Phi$ & $\Phi$ & $\Phi$ & $\Phi$ & \\
\hline $1<-1100$ & 0 & 0 & 0 & $\Phi$ & $\Phi$ & $\Phi$ & $\Phi$ & $\Phi$ & \\
\hline$\hat{\imath} \quad 0010$ & $\Phi$ & $\Phi$ & $\Phi$ & $\Phi$ & 0 & $\Phi$ & $\Phi$ & 0 & \\
\hline $0 \quad 0000$ & \multicolumn{4}{|c|}{ East neighbor } & $\Phi$ & $\Phi$ & $\Phi$ & $\Phi$ & \\
\hline WH WH3:0 & & & & & & & & & \\
\hline
\end{tabular}

West neighbor

(b)

\begin{tabular}{|c|c|c|c|c|c|c|c|c|c|}
\hline $\mathrm{H}+$ & $\begin{array}{c}X \\
000\end{array}$ & ( & $\begin{array}{c}A \\
011\end{array}$ & $\begin{array}{c}\text { ) } \\
010\end{array}$ & $\begin{array}{c}0 \\
100\end{array}$ & $\begin{array}{c}- \\
101\end{array}$ & $\begin{array}{c}- \\
111\end{array}$ & $\begin{array}{c}1 \\
110\end{array}$ & $\begin{array}{c}\text { ET } \\
\text { ET2:0 }\end{array}$ \\
\hline$->0001$ & 0 & 0 & & $0<-$ & $\Phi$ & $\Phi$ & $\Phi$ & & \\
\hline $0<-0100$ & $0<-$ & 0 & 0 & $0<-$ & $\Phi$ & $\Phi$ & $\Phi$ & $\Phi$ & \\
\hline $1<-1100$ & $1<-$ & 0 & 0 & $\Phi$ & $\Phi$ & $\Phi$ & $\Phi$ & $\Phi$ & \\
\hline ^ 0010 & $\Phi$ & $\Phi$ & $\Phi$ & $\Phi$ & 0 & $\Phi$ & $\Phi$ & 0 & \\
\hline $0 \quad 0000$ & 0 & 0 & 0 & 0 & $\Phi$ & $\Phi$ & $\Phi$ & $\Phi$ & \\
\hline EH EH3:0 & & & & & & & & & \\
\hline
\end{tabular}

East neighbor

(c)

Figure 4.14: Head gene computation (sub-program Headgene). (a) Karnaugh map for the central cell. (b) Karnaugh map for the West neighboring cell. (c) Karnaugh map for the East neighboring cell. 


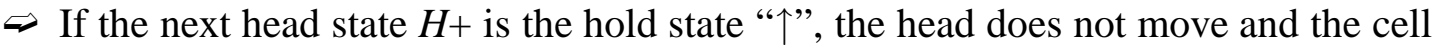
keeps its " $\uparrow "$ " state; we therefore rewrite all the occurrences of $H+=$ " $\uparrow$ " from the original state table of Figure $4.2 \mathrm{~b}$ in the new Karnaugh map of Figure 4.14a. If the next state $H+$ in Figure $4.2 \mathrm{~b}$ is " $\rightarrow$ ", " $0 \longleftarrow$ " or " $1 \leftarrow$ ", the head moves (right or left) and therefore leaves the present square, and the next state $H+$ of the actual cell will be " 0 ". All the dashes of Figure 4.2b become "don't care" conditions $(\Phi)$ in Figure 4.14a. Finally, if the present $H$ state is the quiescent " 0 " state, its next value $H+$ will depend on the next state of its two West and East neighbors ( $W H+$ and $E H+)$.

$\Rightarrow$ A first test is then performed on the West neighbor. All occurrences of the next head state $\mathrm{WH+}=$ " $\rightarrow$ " are rewritten from the original state table of Figure $4.2 \mathrm{~b}$ (depending on the WT2:0 and WH3:0 variables) into the new Karnaugh map of Figure 4.14b which completes Figure 4.14a. Except for the $\Phi$ conditions, all other next states $W H+$ produce the value $H+=$ " 0 " in this new map. Finally, if the West neighbor cell is itself in the quiescent state $W H=$ " 0 ", a last test is performed on the East neighbor.

$\Rightarrow$ All the occurrences of the next head state $E H+=$ " $0 \longleftarrow$ " or " $1 \leftarrow$ " are rewritten from the original state table into the new Karnaugh map of Figure 4.14c, which concludes the description of the next head state $H+$ of the central cell. Except for the $\Phi$ conditions, all other next states $E H+$ produce the value $H+=$ " 0 ".

The three Karnaugh maps of Figure 4.14a, Figure 4.14b, and Figure 4.14c may be simplified and produce seven blocks each, that is, the binary decision tree with 21 branches and the flowchart of the corresponding Headgene sub-program (Figure 4.15).

It is possible to introduce further, and more tedious, simplifications. As an example, the block defined by WH3:O $=0010$ in Figure $4.14 \mathrm{~b}$ requires a test on the variable $W H 1$ in the tree of Figure 4.15. Such a test may be suppressed for the following reason:

$\Rightarrow$ if $W H 3: 0=0010$, the West neighbor cell is in the hold state $W H=\uparrow$ and the next head state $H+$ will be 0 ;

$\Rightarrow$ if this test is ignored, the microprogram will test the East neighbor cell which is obviously in a quiescent state $\left(E H={ }^{\prime \prime}(0)\right.$, producing the same final result : $H+=0$ (remembering that the read/write head has a single position on the tape).

If we suppress the above-mentioned test on the $W H 1$ variable, the final binary decision tree, as well as the corresponding flowchart, consist of 20 branches (Figure 4.15).

Cellular differentiation occurs, in our example, only through the vertical coordinate, computed as a function of the coordinate $S Y$ of the preceding cell (the southern neighbor). From the description of Figure 4.9, the two main genes Headgene for the head cells and Tapegene for the tape cells can be discriminated by the single SYO variable. The final 


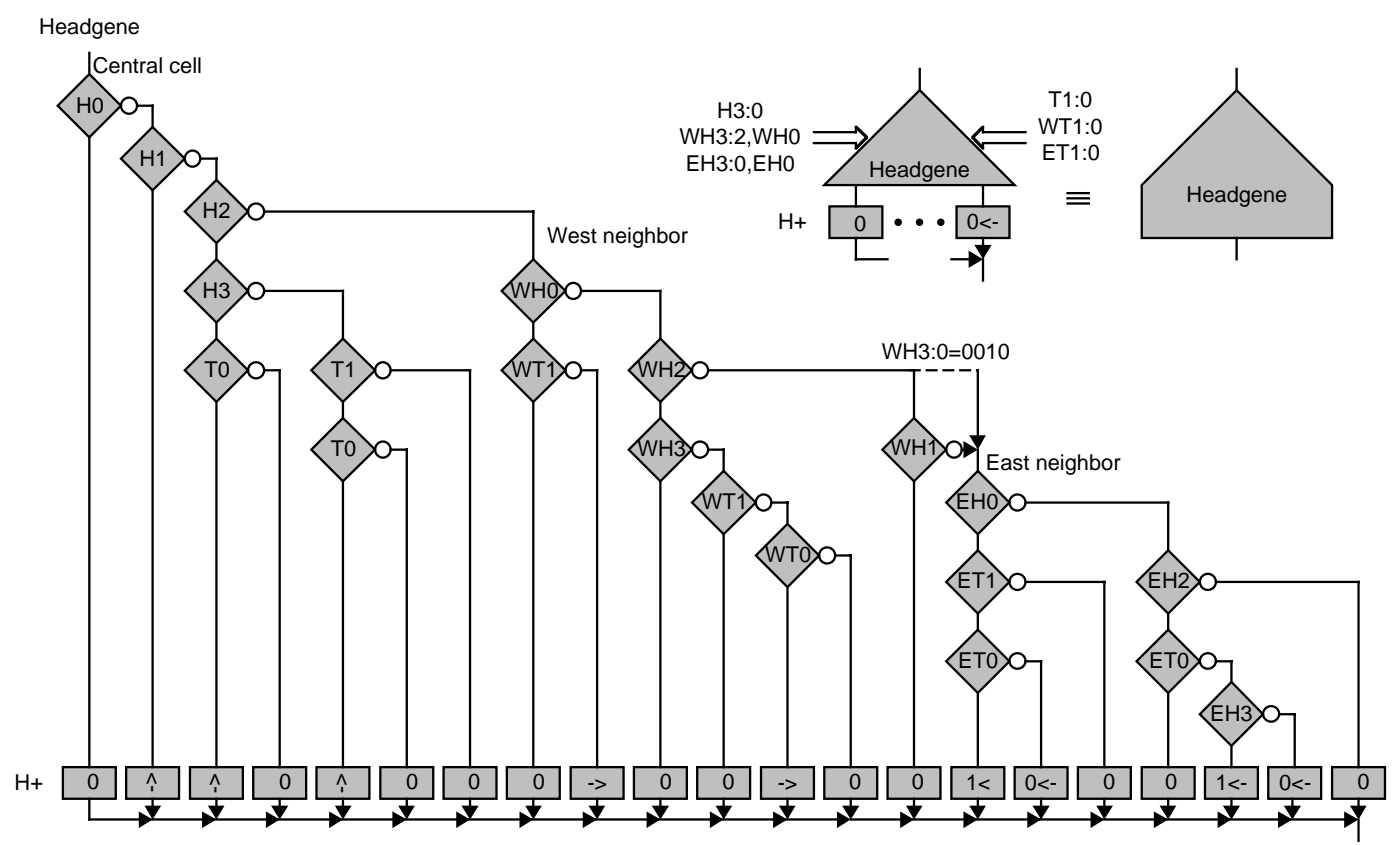

Figure 4.15: Head gene computation (sub-program Headgene): binary decision tree and flowcharts.

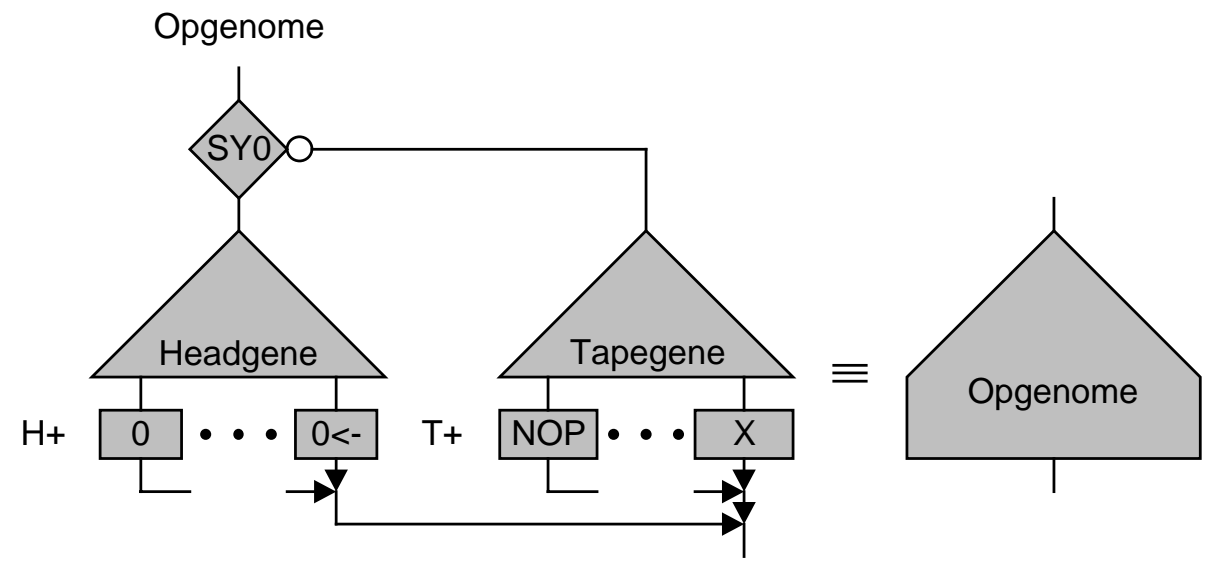

Figure 4.16: Flowcharts of the genome operational part (sub-program Opgenome).

flowchart for the operational part of the genome, Opgenome, which includes the subprograms Headgene and Tapegene, is illustrated in Figure 4.16.

The original specifications of our specialized Turing machine, a parenthesis checker, allowed us to generate five genes, realized by five sub-programs:

$\Rightarrow$ The Xcoord and Ycoord genes, dedicated to the computing of the coordinates, and 
the Initcond gene, required for the calculation of the initial conditions of both the tape states and head states. The Xcoord and Initcond genes depend strongly on the given example, in our case the short "(()" expression.

$\approx$ The Tapegene and Headgene, which calculate the original state table of the parenthesis checker as designed by Minsky. These genes are independent of the chosen example and constitute the invariant part of the final microprogram; they are differentiated by the coordinate variable $S Y O$ and generate the sub-program Opgenome, the operational part of the genome.

It must finally be pointed out that Figure 4.12 illustrates the particular example of Figure 4.8. For any other initial conditions related to the same parenthesis checker, this part of the microprogram must be redesigned to meet the new specifications.

\subsubsection{Configurations and microprogram}

To compute the initial conditions, we need a boolean variable INIT (see the complete microprogram in Figure 4.20). This variable will be introduced in the north side of the cellular space and must reach every cell. We thus have the global configuration of Figure 4.17, described by an assignment instruction:

$$
\text { do } S O 1=N I 1
$$

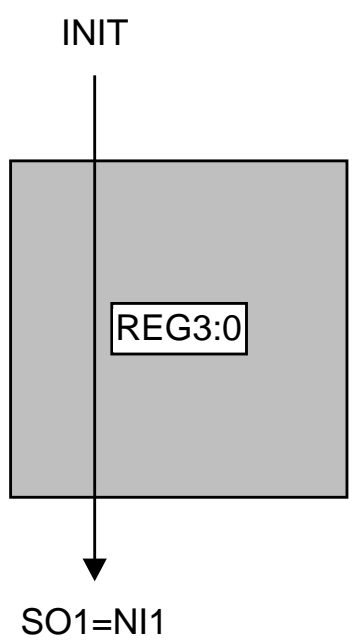

Figure 4.17: Parenthesis checker: global configuration.

The architecture of our Turing machine (Figure 4.9) consists of two cellular rows that are uniform, i.e., characterized by cells with identical structure and behavior (Figure 4.18): 


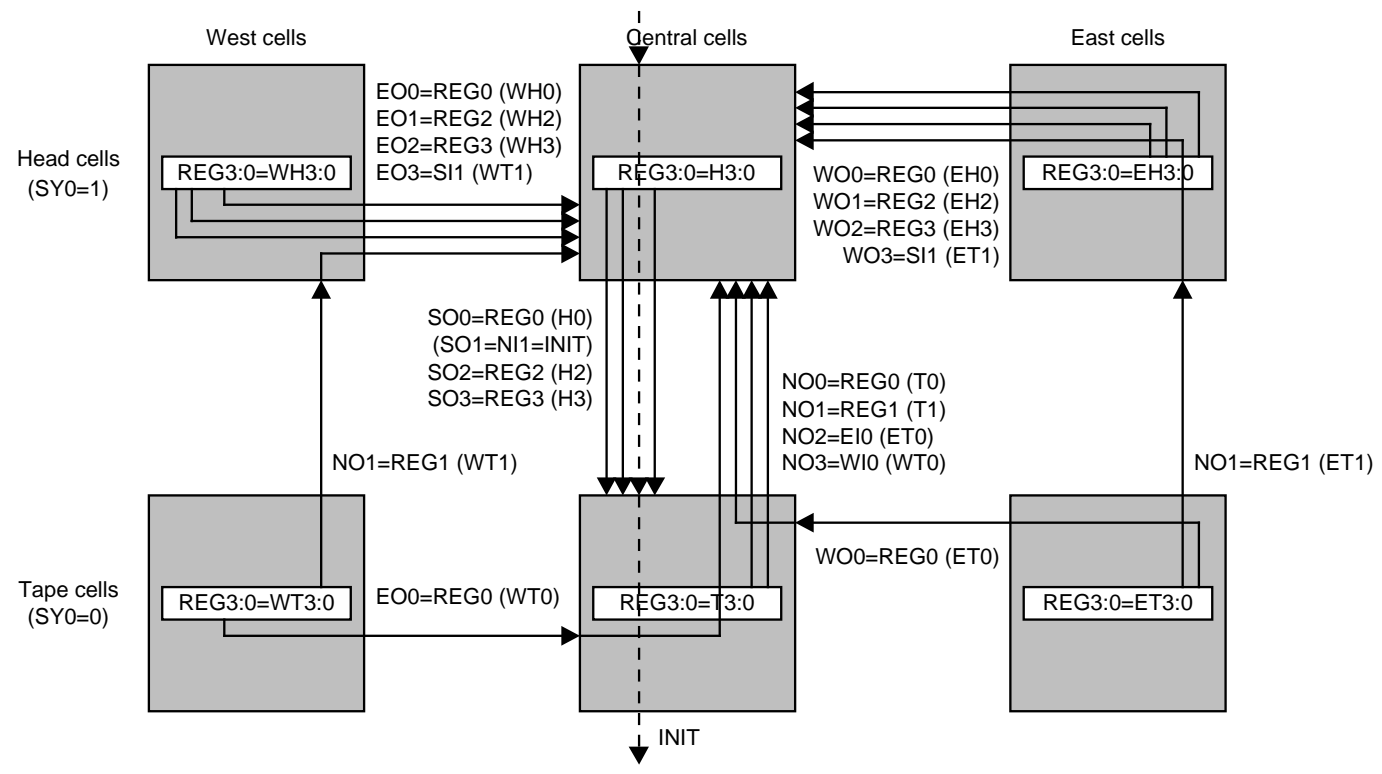

Figure 4.18: Parenthesis checker: local configuration.

$\Rightarrow$ For $S Y O=0(Y=1)$, the tape cells calculate the tape state REG3:0=T3:0. According to Figure 4.13b and Figure 4.15, bits of T3:0 are needed for the calculation of $T+$ itself (but do not need any programmable connection) and for the calculation of the next state $H+$ of the central cell. In this case (Figure 4.15), we need connections from the central tape cell to the central head cell (T1:0 via the bus NO1:0), from the West tape cell to the central head cell (WTO via the EOO and NO3 busses, WT1 via the $N O 1$ bus), from the East tape cell to the central head cell (ETO via the WOO and $N O 2$ busses, ETI via the $N O 1$ bus). The corresponding assignment instructions may be written as follows:

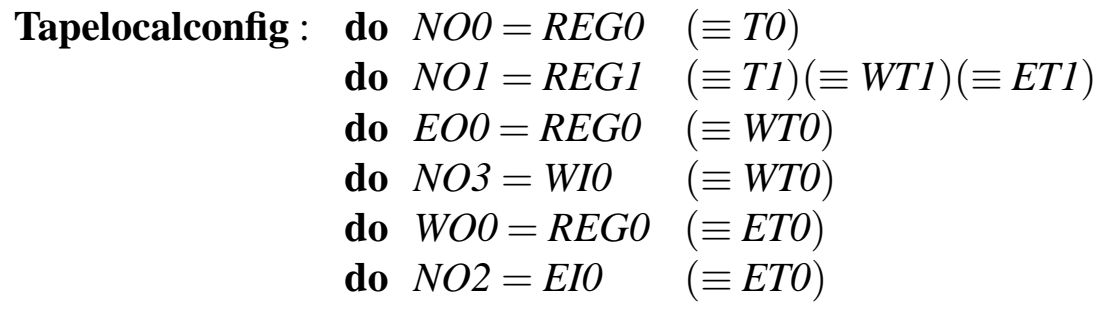

$\Rightarrow$ For $S Y O=1(Y=0)$, the head cells calculate the head state REG3:0 =H3:0. According to Figure 4.13b and Figure 4.15, bits of $H 3: 0$ are needed for the calculation of the next tape state $\mathrm{T+}(\mathrm{H3}: 2$ and $\mathrm{HO}$ via the $\mathrm{SO} 3: 2$ and $\mathrm{SOO}$ busses) and for the calculation of the next state $H+$ of the central cells. In this last case, we need connections from the central head cell itself (H3:O: no programmable connection is needed), from the West head cell to the central head cell (WH3:2,0 via the EO2:0 bus, $W T 1$ via the $E O 3$ bus), from the East head cell to the central head cell (EH3:2,0 
via the WO2:0 bus, ET1 via the WO3 bus). The corresponding assignment instructions may be written as follows:

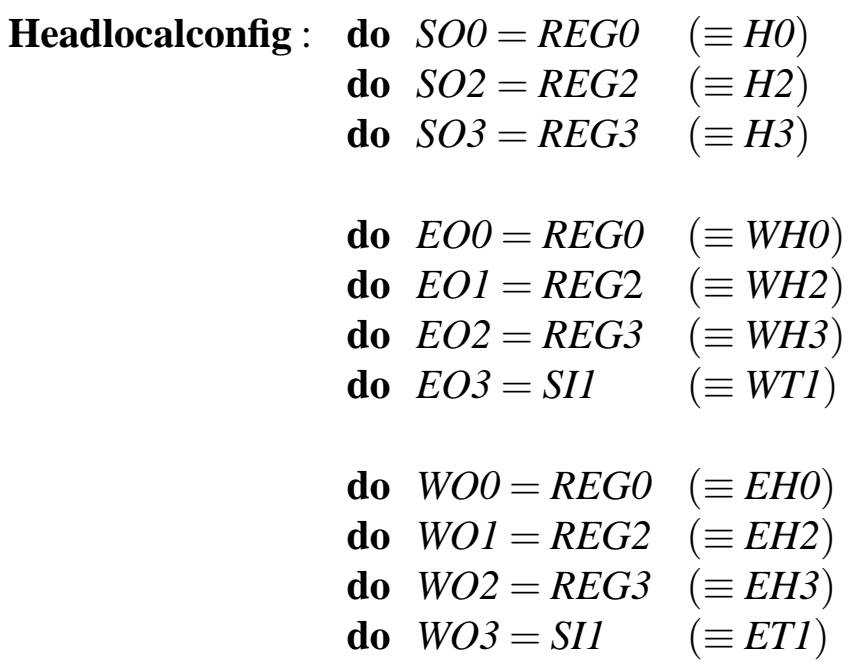

Given the features of the MICTREE cell (Chapter 3), we observe that routing the different busses between head cells and tape cells is not a trivial task; in fact, we remark that, except for the north side, we use all the input/output capabilities of the MICTREE head cells. A minor simplification, such as that mentioned in Subsection 4.4.3 (WH1 test), is of great interest, as the central cell may be independent of the $W H 1$ variable, thus saving one crucial bus connection.

The vertical coordinate $S Y O$ performs the differentiation between the local configuration of tape cells and head cells. It is therefore possible to add to the previous Ycoord sub-program (Figure 4.11b) the new Tapelocalconfig and Headlocalconfig sub-programs determined above. We obtain then the final sub-program (or gene) Ycoordlocalconfig (Figure 4.19), which combines the calculation of $Y$ coordinate and of the local configuration depending on this variable.

The complete genome is represented by the final flowchart Turinggenome of Figure 4.20. It starts with clear conditions assuring that:

$\Rightarrow$ all the state registers of the array are set to $0(R E G=0)$;

$\Rightarrow$ the coordinates $X$ and $Y$ are set to $0(X=0, Y=0)$.

The microprogram then executes the single global configuration instruction $(S O 1=N I 1)$ and, for $I N I T=1$, the left loop (initialization loop). If $K$ is the largest value for either the $X$ or the $Y$ coordinate (according to Figure 4.9, $K=6$ in our example), the left-hand loop must be executed at least $K=6$ times: at the start of the microprogram, or when a repair involving a change of coordinates occurs, the coordinates are recomputed starting from the mother cell (with $W X, S Y=00$ ). At least $K$ executions 


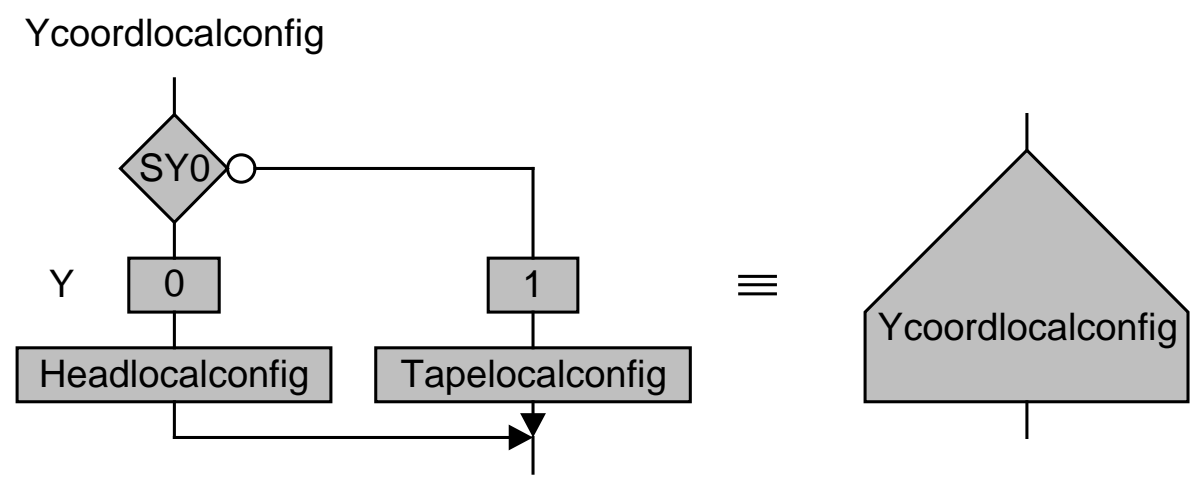

Figure 4.19: Flowcharts for $Y$ coordinate and local configuration calculation (Ycoordlocalconfig sub-program).

of the left-hand loop are necessary to ensure that the right-most (or upper-most) cell computes the correct coordinates. This computation occurs in the sub-programs Xcoord and Ycoordlocalconfig, and is immediately followed by the calculation of the initial conditions (Initcond).

When the initial conditions have been computed and/or recomputed after self-repair, it is possible to set INIT $=0$; the microprogram executes either the middle or the right loop, depending on the value of the $G$ variable, the global clock signal charged with synchronizing the Turing machine. Each rising edge of $G$ allows the transition from a present tape state $T$ or head state $H$ to a next tape state $T+$ or head state $H+$.

The right-hand loop, the operational part of the genome (Opgenome), is executed once every period of the global clock signal $G$. To assure the synchronization of all the cells, tests are performed throughout the half-period when $G=0$, but no assignment is made until the rising edge of $G(G=0 \rightarrow 1)$, when all the registers $R E G$ (i.e., the $H$ and $T$ states of the cells) are updated simultaneously.

\subsection{Conclusion}

In this chapter we showed that, based on the three fundamental features of Embryonics, the MICTREE artificial cell (Chapter 3) roughly mimics the process of cellular development. Using a binary decision machine as processor, each MICTREE cell executes the genome microprogram of the artificial organism; the whole array is thus able to embed a specialized Turing machine with self-repair and self-replication capabilities. We then presented, in Section 4.3 and Section 4.4, the complete implementation process of a specialized Turing machine, a parenthesis checker.

Our parenthesis checker was fully implemented using MICTREE artificial cells (Figure 3.2). In this realization the self-repair (Figure 4.6) and self-replication (Figure 4.7) 


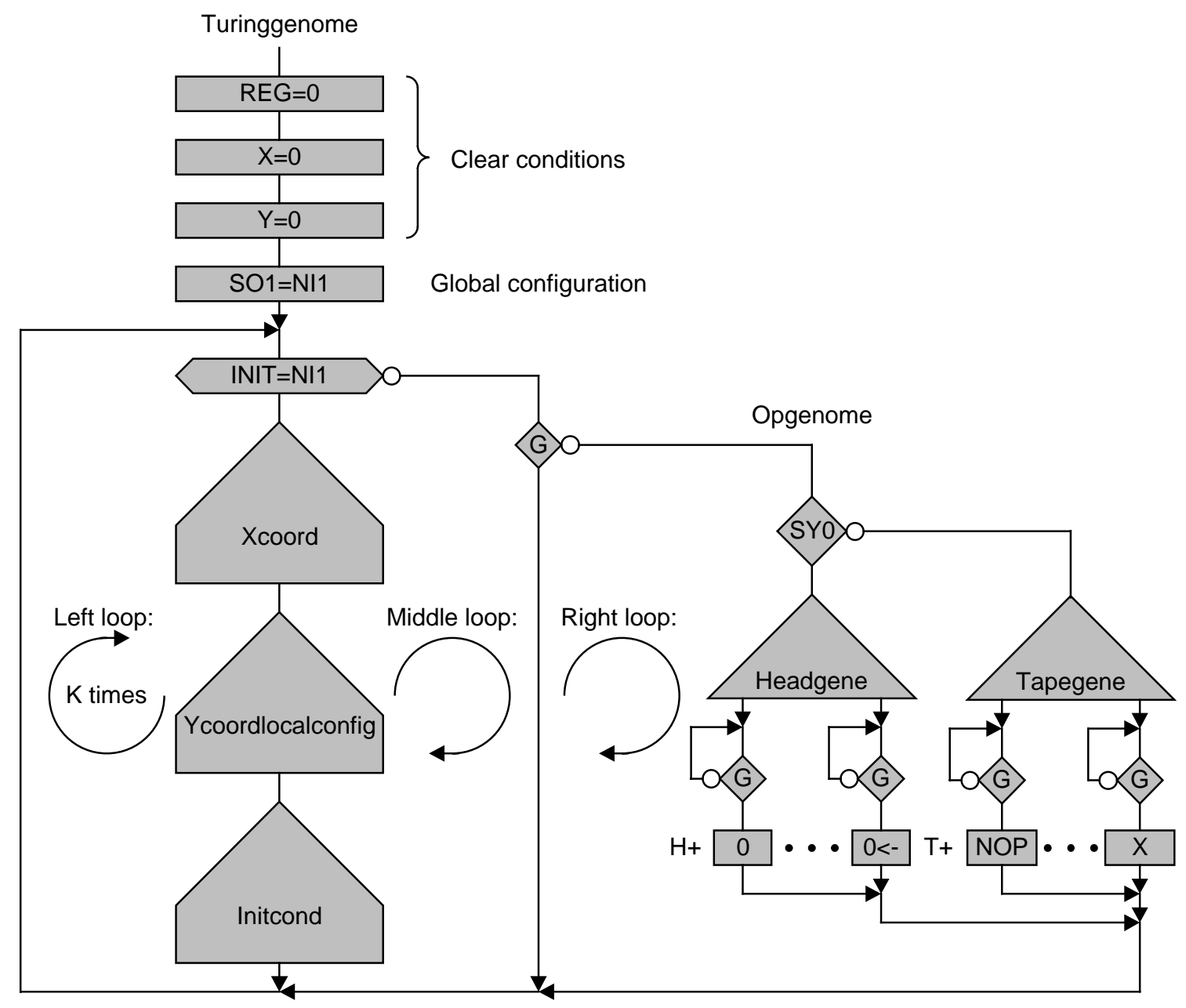

Figure 4.20: Flowchart of the complete genome microprogram (Turinggenome).

capabilities were completely verified.

In the next chapter we will introduce the universal Turing machine (UTM), which is a Turing machine capable of simulating the operation of any specialized Turing machine. We will also show that a hardware implementation of a UTM is possible thanks to the Embryonics architecture. 



\section{Chapter 5}

\section{Universal Turing Machine}

Looking (and dreaming) toward the future, we are working on a multicellular universal Turing machine implementation with self-replication and self-repair capabilities using the MICTREE cell. Our first results around this idea were presented in the Seventh International Conference on Artificial Life [87, 88,90].

Our goal in this chapter is to present self-replicating and self-repairing machines exhibiting universal computation $[87,88,90]$, i.e., universal Turing machines. We demonstrate that the dream of von Neumann, the self-replication of such a machine, can be realized in actual hardware thanks to the Embryonics architecture.

In Section 5.1 we present a brief reminder of the universal Turing machine. In Section 5.2 we introduce the basics of the Embryonics architecture based on a multicellular array of cells. We next present the architecture of an ideal and of an actual universal Turing machine able to self-replicate. In Section 5.3 we then describe the PICOPASCAL language and a hardware implementation of a PICOPASCAL interpreter, which is necessary for the understanding of our Embryonics implementation. Section 5.4 describes the detailed implementation of a self-replicating universal Turing machine. A discussion of our results follows in the final section (Section 5.5).

\subsection{The universal Turing machine}

Turing had the further idea of the universal Turing machine (UTM), capable of simulating the operation of any specialized Turing machine, and gave an exact description of such a UTM in his paper [109]. The importance of the universal Turing machine is clear. We do not need to have an infinity of different machines doing different jobs. A single one will suffice [32].

A universal Turing machine, $U$, is a Turing machine with the property of being able to read the description (on its tape) of any other Turing machine, $T$, and to behave as $T$ would have. The machine $U$ consists of a finite-state machine (the program of $U$ ) 
controlling a mobile head, which operates on a tape. The data on the tape completely describe the machine $T$ to be simulated (the data of $T$ and the program of $T$, i.e., the three functions $Q+, S+$, and $D+\operatorname{describing} T$ according to Section 4.1).

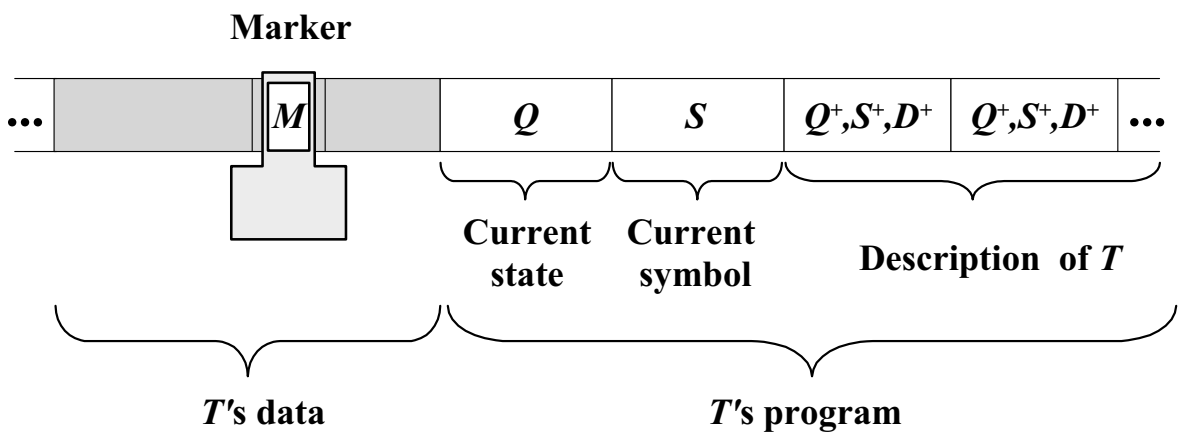

Figure 5.1: Universal Turing machine's tape, describing the specialized machine $T$.

Figure 5.1 shows the organization of $U$ 's tape. To the left is a semi-infinite region containing the data of $T$ 's tape. Somewhere in this region is a marker $M$ indicating where $T$ 's head is currently located. The middle region contains the current internal state $Q$ and the current input symbol $S$ of $T$. The right-hand region is used to record the description of $T$, i.e., the three functions $Q+, S+$, and $D+$ for each combination of $Q$ and $S$.

\subsection{Self-replication of a universal Turing machine on a multicellular array}

The preceding chapter presented a self-replicating two-dimensional artificial organism implementing a specialized Turing machine, the parenthesis checker, realized with ten MICTREE artificial cells. By using the same type of cells we now show how it is possible to design and build a universal Turing machine (UTM) with self-replication and selfrepairing capabilities.

\subsubsection{Multicellular architecture of a universal Turing machine}

Conventional universal Turing machines [64] consist of a finite but arbitrarily long tape, and a single read/write mobile head controlled by a finite-state machine, which is itself described on the tape (Figure 5.1). In order to implement a universal Turing machine in an array of MICTREE artificial cells, we made three fundamental architectural choices (Figure 5.2):

1. The read/write head is fixed; the tapes are therefore mobile. 


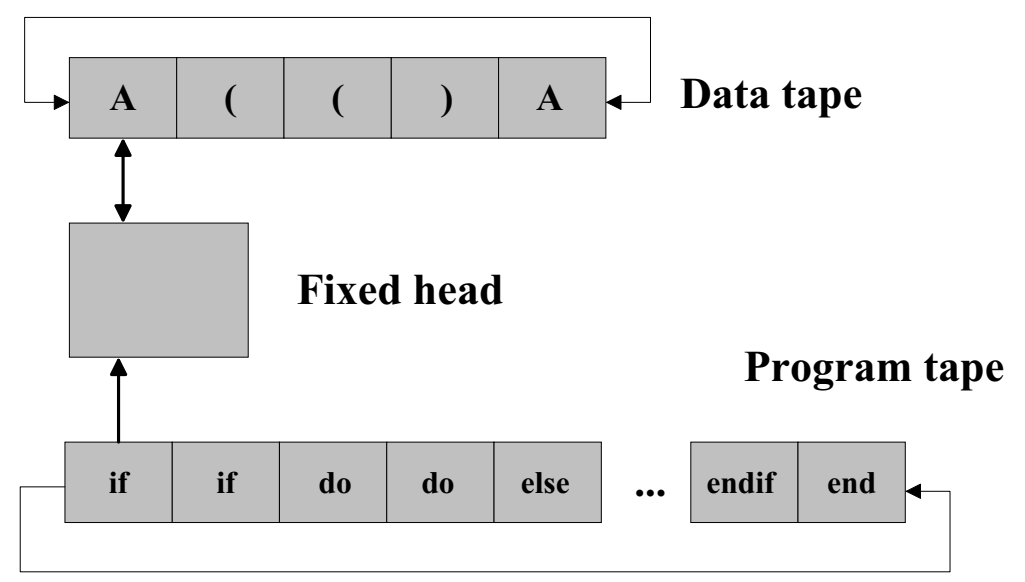

Figure 5.2: Universal Turing machine architecture for the parenthesis checker example.

2. The data of the given application (the specialized Turing machine to be simulated) are placed on a mobile tape, the data tape; this tape can shift right, shift left, or stay in place.

3. The finite-state machine for the given application is translated into a very simple program written in a language called PICOPASCAL (Section 5.3); each instruction of this program is placed in a square of a second mobile tape, the program tape; this tape just needs to shift left. The transformation of a state table into such a program is directly inspired by the $\mathrm{W}$-machine [112] with the major contribution of avoiding the jumps required by the if 1 then $(n)$ else (next) instructions.

The fixed head, which is in fact an interpreter of the PICOPASCAL language, has to continuously execute cycles consisting of four operations:

1. reading and decoding an instruction on the program tape;

2. reading a symbol on the data tape;

3. interpreting the current instruction, and writing a new symbol on the current square of the data tape;

4. shifting the data tape (left, or right, or not at all) and the program tape (left).

\subsubsection{An application: A binary counter}

In order to test our UTM implementation, we used, as a simple but non-trivial example, a binary counter [64], a machine that writes out the binary numbers $1,10,11,100$, etc., the 
size of the numbers being limited only by the dimensions of the data tape. The counter's state table (Figure 5.3) has two internal states $(Q \in\{0 \rightarrow, 1 \leftarrow\})$ and two input states $(S$ $\in\{0,1\}), S$ being the value of the current square read on the data tape. In this example we combined the internal state $Q$ with the direction of the tape. For $Q=\{0 \rightarrow\}$ the data tape will move to the right, for $Q=\{1 \leftarrow\}$ the data tape will move to the left. Depending on the present internal state $Q$ and the present input state $S$, the specialized Turing machine will:

1. write a new binary value $S+(0,1)$ on the current square of the data tape;

2. move its data tape to the right $\left(Q_{+}=0 \rightarrow\right)$ or to the left $(Q+=1 \leftarrow)$, which is equivalent to moving a mobile head to the left or to the right, respectively;

3. go to the next state $Q+(0 \rightarrow, 1 \leftarrow)$.

\begin{tabular}{|c|c|c|}
\hline$Q+, S+$ & $S=0$ & $S=1$ \\
\hline$Q=0 \rightarrow$ & $0 \rightarrow, 0$ & $1 \leftarrow, 1$ \\
\hline$Q=1 \leftarrow$ & $0 \rightarrow, 1$ & $1 \leftarrow, 0$ \\
\hline
\end{tabular}

Figure 5.3: State table of the binary counter.

The PICOPASCAL program equivalent to the state table (Figure 5.3) is given in Figure 5.4.

\subsubsection{An ideal architecture for the universal Turing machine}

A universal Turing machine architecture, ideal in the sense that it is able to deal with applications of any complexity, is characterized by:

1. a finite, but arbitrarily long data tape;

2. a read/write head able to interpret a PICOPASCAL program of any complexity;

3. a finite, but arbitrarily long program tape.

It must be pointed out that, for any application, the program tape and the read/write head (the PICOPASCAL interpreter) are always characterized by finite and defined dimensions; only the data tape can be as long as desired, as is the case for the binary counter, whose growth is potentially infinite.

An ideal architecture, embedding the current example, but compatible with any other application, could be the following (Figure 5.5): 


\begin{tabular}{|c|c|c|}
\hline ADR & DATA & PROGRAM \\
\hline 00 & 5 & if $(Q)$ \\
\hline 01 & 5 & if $(S)$ \\
\hline 02 & $\mathbf{A}$ & do $0 \quad(S)$ \\
\hline 03 & 9 & do $1 \leftarrow(Q)$ \\
\hline 04 & 4 & else \\
\hline 05 & B & do 1 (S) \\
\hline 06 & 8 & do $0 \rightarrow(Q)$ \\
\hline 07 & 6 & endif \\
\hline 08 & 4 & else \\
\hline 09 & 5 & if $(S)$ \\
\hline $\mathbf{0 A}$ & B & do 1 (S) \\
\hline $\mathbf{0 B}$ & 9 & do $1 \leftarrow(Q)$ \\
\hline $\mathbf{0 C}$ & 4 & else \\
\hline OD & $\mathbf{A}$ & do $0 \quad(S)$ \\
\hline $\mathbf{O E}$ & 8 & do $0 \rightarrow(Q)$ \\
\hline $\mathbf{O F}$ & 6 & endif \\
\hline 10 & 6 & endif \\
\hline 11 & 2 & end \\
\hline
\end{tabular}

Figure 5.4: PICOPASCAL program equivalent to the state table of Figure 5.3.

1. The data tape, able to shift right, to shift left, or hold, is folded on itself. The initial state is defined in Figure 5.5 by $Q L 3: 0, Q C, Q R 0: 3=000010000$, where $Q L$ are the squares to the left of the central square $Q C$, and $Q R$ are the squares to the right of $Q C$; the data tape is able to grow to the left of $Q C$, i.e., to the right of $Q L O(Q L 4, Q L 5, \ldots)$ and to the right of $Q C(Q R 4, Q R 5, \ldots)$, as can be appreciated in Figure 5.5.

2. The fixed read/write head, which will be detailed in Section 5.4, is basically composed of a state register $Q, S$ (storing the current values of internal and input states $Q, S$, respectively, with an initial state $Q, S=01$ ) and a stack $S T 1: 3$ characterized by a 1-out-of-3 code (one-hot encoding). At the start of the execution of the PICOPASCAL program (i.e., in Figure 5.4, at address $A D R=00$ ), the stack is in an initial state $S T 1: 3=100$. Roughly speaking, each if instruction will involve a PUSH operation, each endif a POP operation, and each else a LOAD operation. When $S T 1=1$, the do instructions are executed. The main characteristic of the stack is its scalability: for any program exhibiting $n$ nested if instructions, the stack is organized as a $n+1$ squares shift register. Both the $S T 1: 3$ stack and the $Q, S$ register are able to grow to accommodate more complex applications.

3. The program tape is folded on itself; it is able to grow to accommodate more complex applications. 

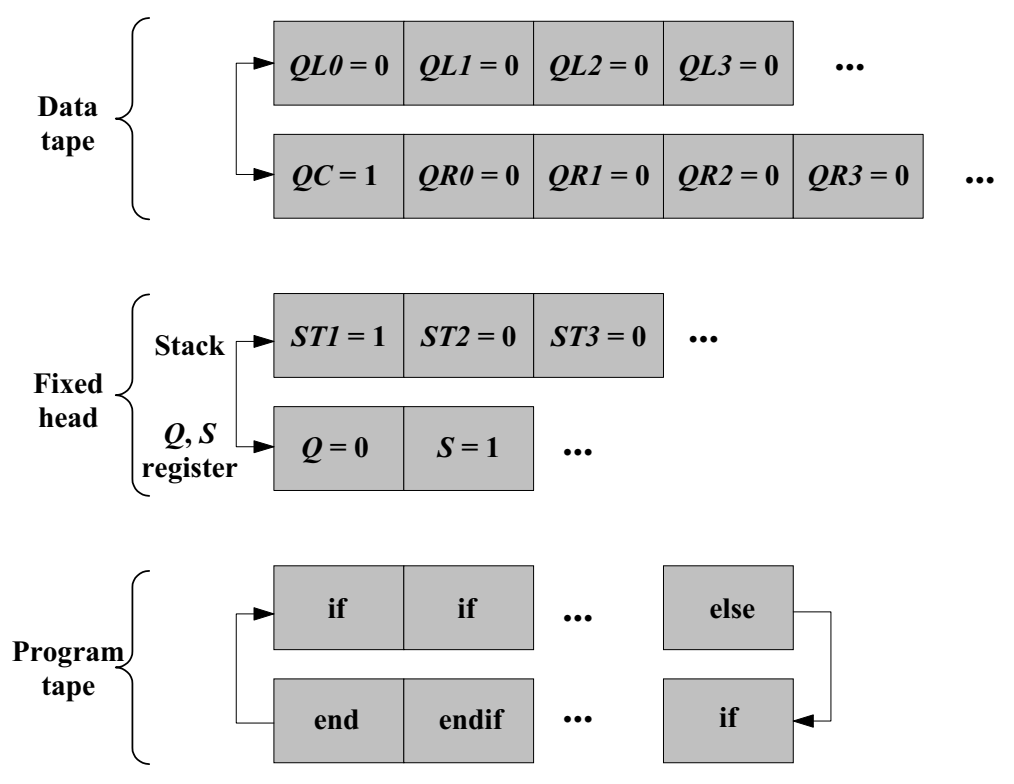

Figure 5.5: UTM's ideal architecture.

\subsubsection{An actual implementation of the UTM for the binary counter example}

In order to implement the binary counter application with a limited number of MICTREE artificial cells, we have somewhat relaxed the requirements of the ideal architecture described earlier. Our final architecture is made up of three rows $(Y=1 \ldots 3)$ and nine columns $(X=1 \ldots 9)$ organized as follows (Figure 5.6):

$\Rightarrow$ The 18 instructions of the PICOPASCAL program (Figure 5.4) are placed in the program tape, using the two lower rows $(Y=1,2)$ of the array.

$\Rightarrow$ The read/write head is composed of a $S T 1: 3$ stack and of the $Q, S$ register $(X=1 \ldots 5$, $Y=3)$, while the data tape is implemented by three cells $(X=6 \ldots 8, Y=3)$ storing 9 bits $Q L 3: 0, Q C, Q R 0: 3$.

In order to demonstrate self-repair, we added spare cells to each row, at the right-hand side of the UTM, all identified by the same horizontal coordinate ( $X=9$ in Figure 5.6). As previously mentioned, more cells may be used not only for self-repair, but also for a UTM necessitating a growth of the tape of arbitrary, but finite, length.

Self-replication rests on two hypotheses (Figure 5.7):

$\Rightarrow$ there exist a sufficient number of spare cells (unused cells at the upper side of the array, at least $3 \times 9=27$ for our example); 


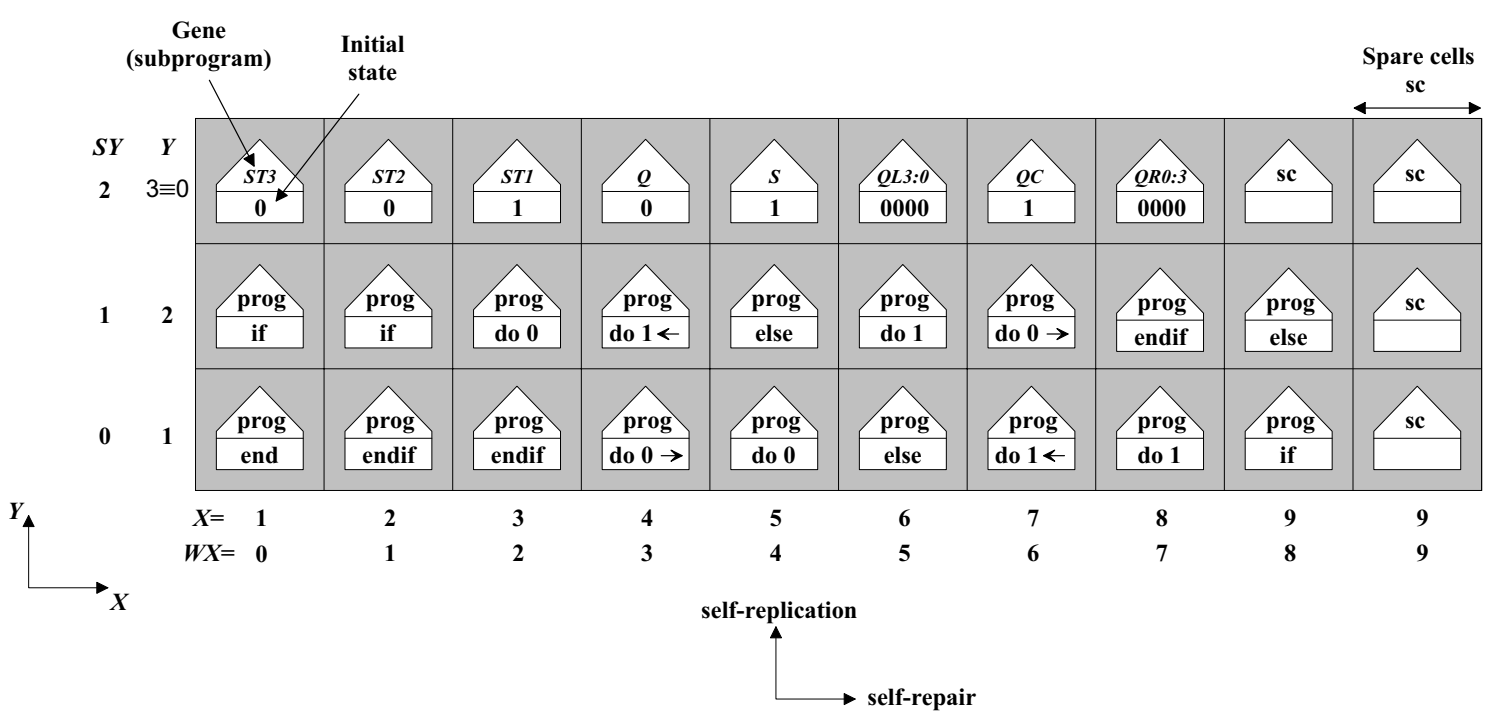

Figure 5.6: UTM's actual implementation for the binary counter example on a multicellular array of 27 MICTREE cells plus 3 spare cells (sc). $W X$ : horizontal coordinate of the western neighboring cell; $S Y$ : vertical coordinate of the southern neighboring cell.

$\Rightarrow$ the calculation of the coordinates produces a cycle at the cellular level (in our example: $Y=1 \rightarrow 2 \rightarrow 0 \rightarrow 1 \rightarrow 2 \rightarrow 0$ ).

Given a sufficiently large space, the self-replication process can be repeated for any number of specimens in the $Y$ axis. With a sufficient number of cells, it is obviously possible to combine self-repair (or growth) towards the $X$ direction and self-replication towards the $Y$ direction.

In next section we will present the PICOPASCAL language and a PICOPASCAL interpreter architecture, which are necessary for the understanding of our Embryonics implementation. Then in Section 5.4 we will describe the detailed implementation of the self-repairing and self-replicating universal Turing machine. 


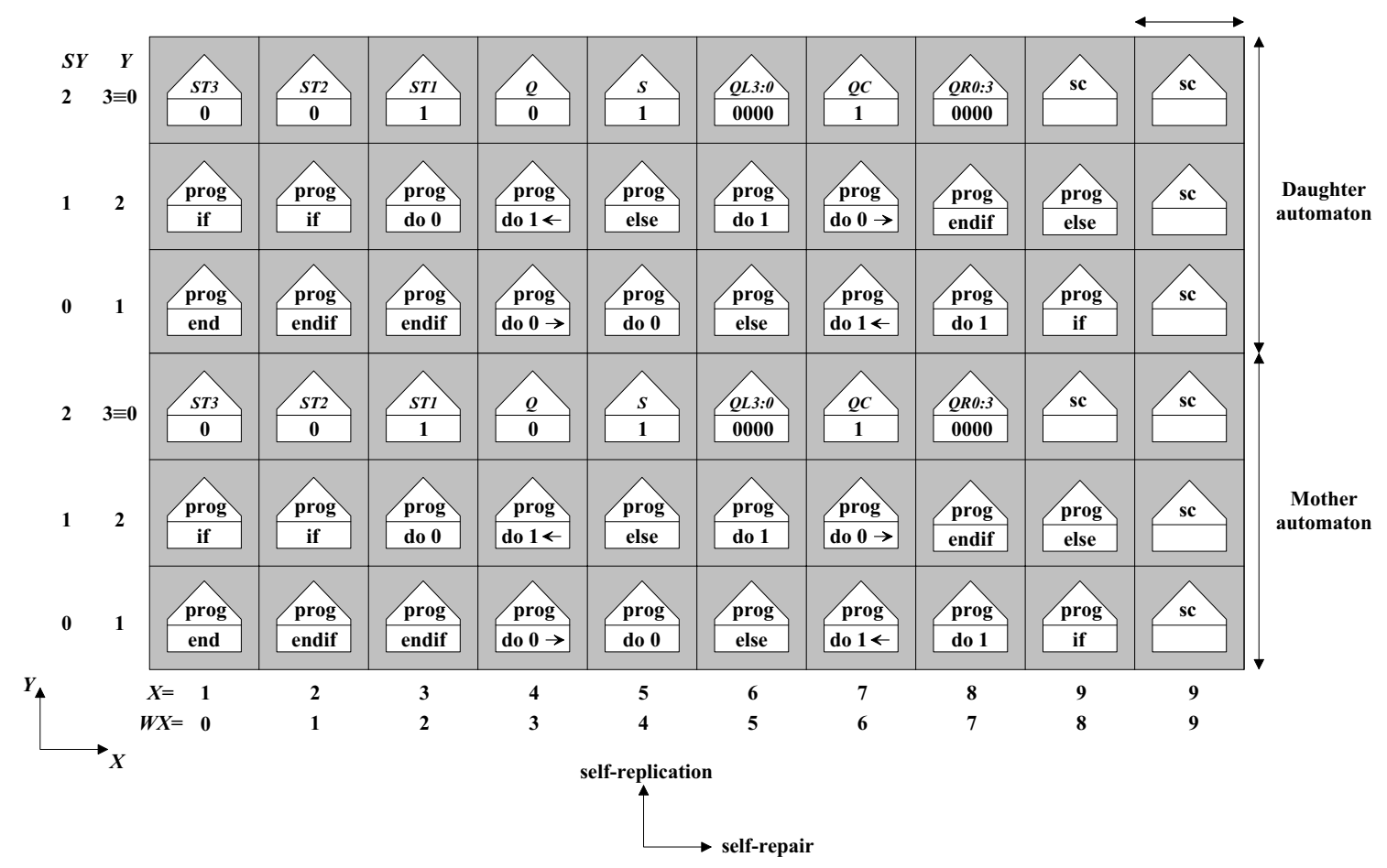

Figure 5.7: Self-replication of the UTM's actual implementation for the binary counter example. WX: horizontal coordinate of the western neighboring cell; SY: vertical coordinate of the southern neighboring cell.

\subsection{PICOPASCAL}

In this section we present the PICOPASCAL language and a possible hardware architecture for a PICOPASCAL interpreter. The PICOPASCAL language and its interpreter have been customized for our Embryonics architecture and the UTM implementation described in Section 5.4.

\subsubsection{The PICOPASCAL language}

The PICOPASCAL language consists of a minimal subset of the MODULA-2 language [114]. PICOPASCAL is thus a high-level language: it does not make use of explicit addressing and provides a great simplicity of use. PICOPASCAL is, moreover, a structured language and thus guarantees, because of its structure, a rigorous and efficient notation. In conformity with this last feature, PICOPASCAL has three fundamental constructs, described below: (1) the sequence, (2) the choice or alternative, and (3) the iteration. 


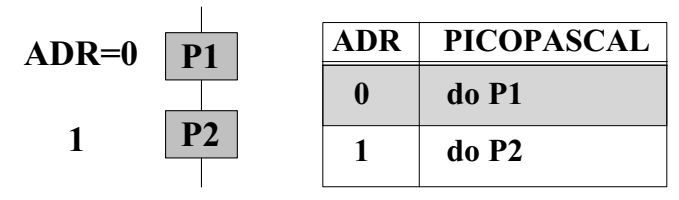

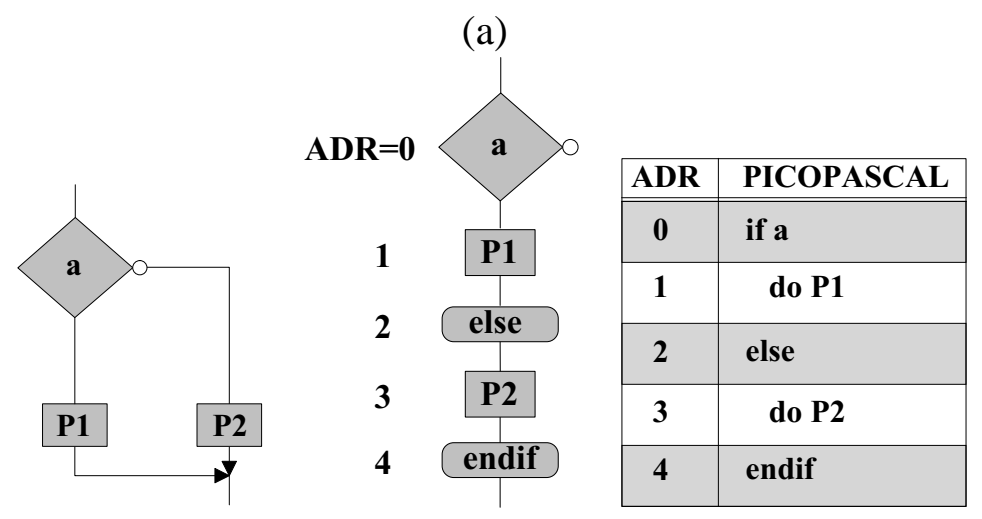

(b)

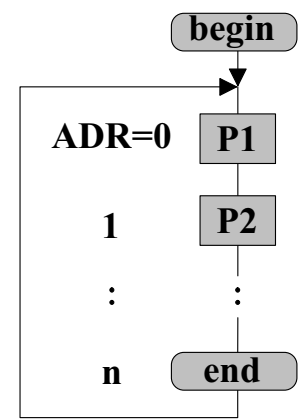

(c)

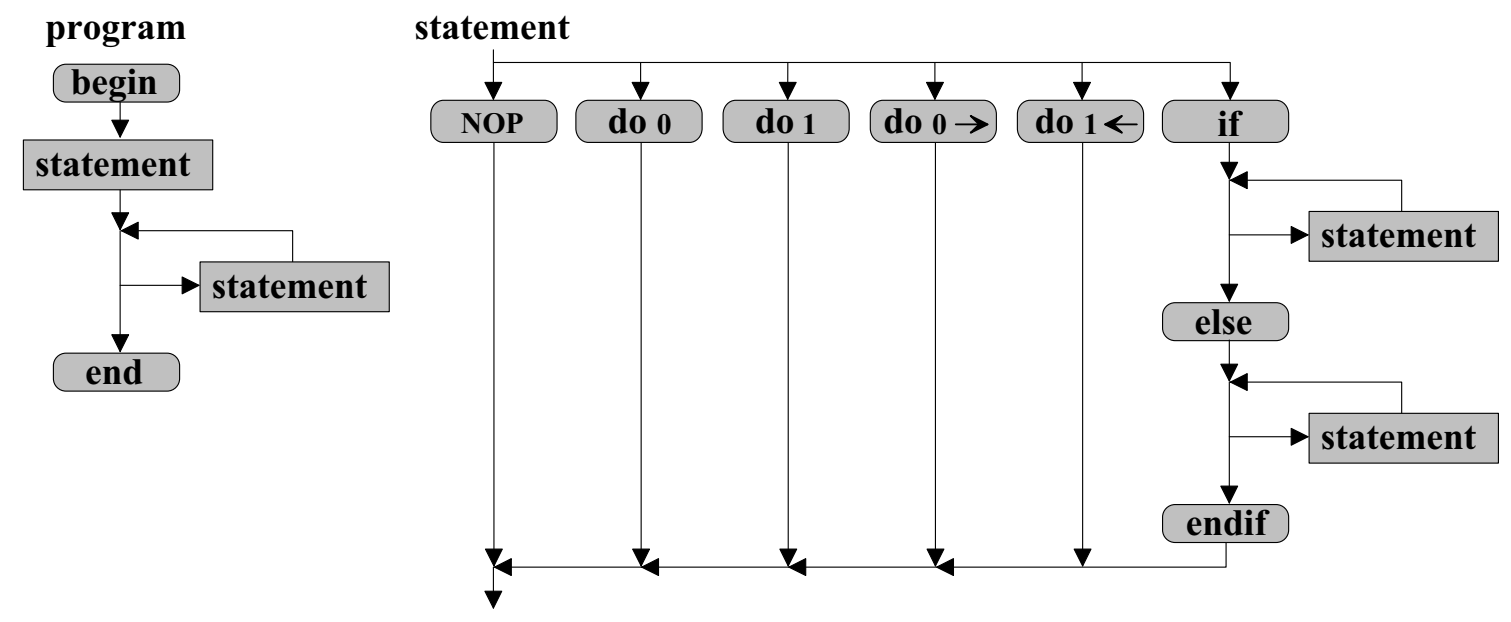

(d)

Figure 5.8: PICOPASCAL language. (a) Sequence of two assignment instructions: do P1P2. (b) Choice of either $\mathrm{P} 1$ or $\mathrm{P} 2$ : if $a$ then $\mathrm{P} 1$ else $\mathrm{P} 2$. (c) Non-conditional iteration loop. (d) Syntactic diagram. 
The assignment do..., realizing the synchronous transfer of a constant into a register, is a structured program. The sequence (or composition) of two such instructions $P l$ and $P 2$, written do $P 1 P 2$, is a structured program, described by the flowchart and by the mnemonic program of Figure 5.8a. This last notation consists of a linear succession of instructions, displayed in the growing order of addresses $A D R$.

The choice (or alternative) of $P 1$ or $P 2$, where $P 1$ and $P 2$ are two assignments, is a structured program, written if $a$ then $P 1$ else $P 2$. It is represented symbolically by the flowchart of Figure 5.8b, and realized by the linear succession of the instructions of the corresponding functional diagram and mnemonic program. To facilitate comprehension, and unlike programs written in a low-level language using explicit addresses, there is no jump (notably, to avoid the instruction $P 1$ when $a=0$ or the instruction $P 2$ when $a=1$ ): all instructions are read sequentially, from $A D R=0$ to $A D R=4$, and the execution of the assignments $P 1$ or $P 2$ depends on the value of a signal EXEC (for EXECUTE) which, in turn, depends on the value of the test variable $a$. This process will be revisited in detail in the description of the interpreter of the PICOPASCAL language (Subsection 5.3.2).

The last construct of structured programming, the conditional iteration while $a$ do $P 1$, is thus not necessary in the PICOPASCAL language. However, since our program must be continually executed, notably to allow self-repair, we allow the loop illustrated by the flowchart of Figure 5.8c, which in fact introduces a non-conditional iteration on the entire program.

In conclusion, the PICOPASCAL language is described by the syntactic diagram of Figure 5.8d, where we can count ten different terminal symbols (ovals), which make up the instructions of the language: begin, end, NOP, do 0 , do 1 , do $0 \rightarrow$, do $1 \leftarrow$, if, else, endif. The NOP (No operation) instruction represents the execution of a neutral operation.

Figure 5.9a shows the operating code $(O P C)$ for the instructions of the PICOPASCAL language. Figure 5.9b and Figure 5.9c show the binary decision diagram of the binary counter example and its PICOPASCAL description, derived from the state table in Figure 5.3.

\subsubsection{PICOPASCALINE: an interpreter for the PICOPASCAL language}

Figure 5.10 suggests a possible hardware architecture to execute the ten instructions of the PICOPASCAL language. From now on, we will refer to this machine as PICOPASCALINE. The instruction end, as well as the pseudo-instruction begin (not executed), have the same effect: jumping to the instruction at address 0 (note that in this architecture the begin instruction is not necessary and can thus be removed). The if instruction does not require a test variable, since the hardware is capable of presenting the correct variable at the right time. There exist therefore nine distinct types of instruction to interpret. 


\begin{tabular}{|c|c|l||c|c|c|}
\hline OPC3:0 & OPC & Instruction & OPC3:0 & OPC & Instruction \\
\hline \hline 0000 & $\mathbf{0}$ & NOP & 1000 & $\mathbf{8}$ & do 0 $\rightarrow$ \\
\hline 0001 & $\mathbf{1}$ & begin & 1001 & $\mathbf{9}$ & do 1 \\
\hline 0010 & $\mathbf{2}$ & end & 1010 & A & do 0 \\
\hline 0011 & $\mathbf{3}$ & & 1011 & B & do 1 \\
\hline 0100 & 4 & else & 1100 & C & \\
\hline 0101 & $\mathbf{5}$ & if & 1101 & D & \\
\hline 0110 & $\mathbf{6}$ & endif & 1110 & E & \\
\hline 0111 & 7 & & 1111 & F & \\
\hline
\end{tabular}

(a)

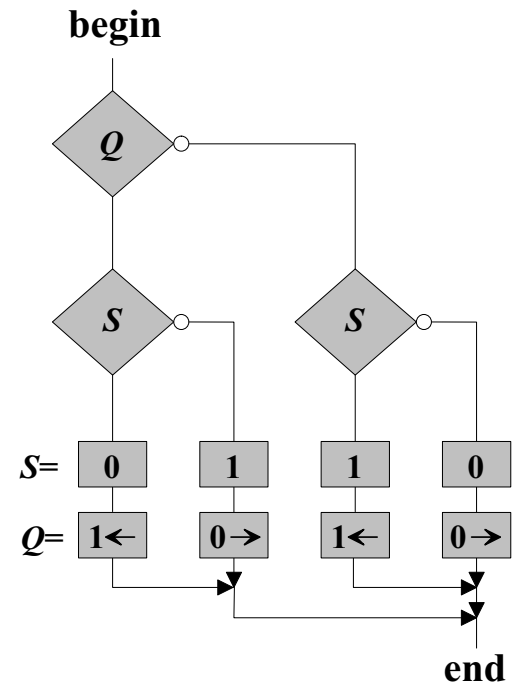

(b)

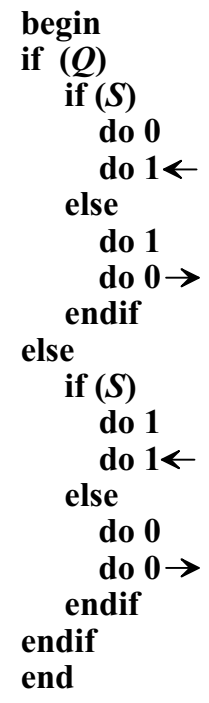

(c)

Figure 5.9: PICOPASCAL language. (a) Opcodes for the ten instructions of the PICOPASCAL language. (b) Binary decision diagram of the binary counter example. (c) PICOPASCAL description.

To decode the instructions (OPC3:0) on the program tape, the PICOPASCALINE consists of the following elements (Figure 5.10):

A state register REGISTER storing the current values of the internal and input states $Q$, and $S$ respectively, with an initial state $Q, S=01$.

$\Rightarrow$ A register REGISTER storing the values $Q L 3: 0, Q C, Q R 0: 3$ of the data tape, with an initial state $Q L 3: 0, Q C, Q R 0: 3=000010000$.

$\Rightarrow$ A stack STACK characterized by a 1-out-of-3 code (one-hot encoding), with an initial state $\mathrm{STACK}=S T 3: 1=001$.

$\Rightarrow$ A decoder DMUX1 controlled by the 4 bits of the operating code OPC3:0, which generates the signals controlling the STACK (signals $I F, E L S E$, and ENDIF). 


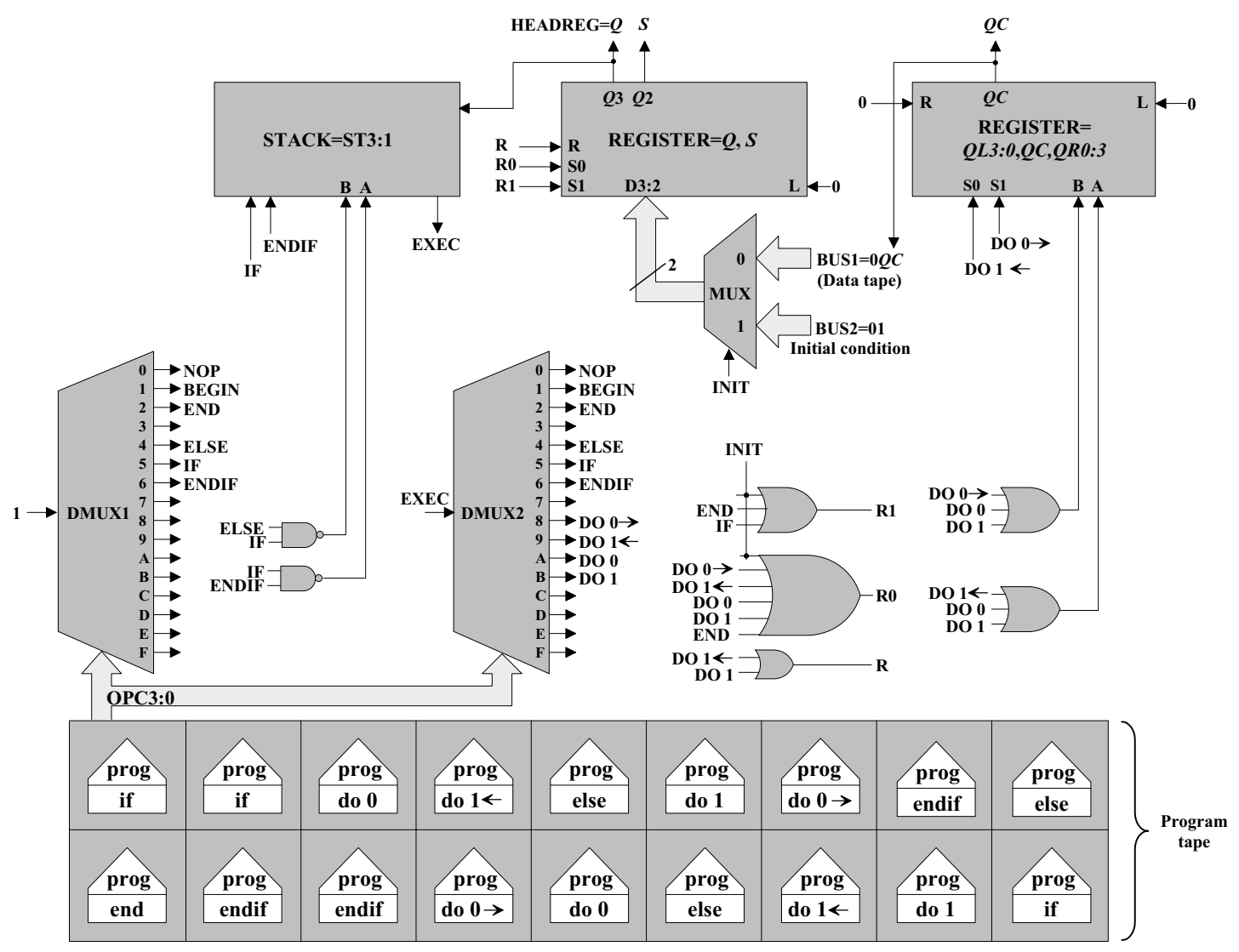

Figure 5.10: PICOPASCALINE: PICOPASCAL interpreter for the ten instructions of the language.

$\Rightarrow$ A decoder DMUX2 controlled by the 4 bits of the operating code OPC3:0 and by the EXEC signal. This decoder generates the signals controlling the $(Q, S)$ and ( $Q L 3: 0, Q C, Q R 0: 3$ ) REGISTERs (signals $D O 0, D O 1, D O 0 \rightarrow, D O 1 \leftarrow, I F$, and ELSE).

$\Rightarrow$ A multiplexer MUX controlled by the signal INIT, which selects one of the two input busses, BUS1 coming from the data tape, or BUS2 which is a constant used for initialization purposes. At the start of the execution the signal INIT has the value 1 and the $(Q, S)$ REGISTER is initialized, whereas the rest of the execution this variable takes the value 0 and the value $Q C$ coming from the ( $Q L 3: 0, Q C, Q R 0: 3)$ REGISTER is assigned to the $(Q, S)$ REGISTER.

The signal EXEC controls the execution of the assignment instructions do and thus depends on the succession of values of the internal and input states $Q$ and $S$. We will now examine this process for the example of the program of Figures 5.4 and 5.9, whose detailed execution is shown in Figure 5.11. We assume that the values of the test variables are $Q=1$ and $S=0$, and that these values do not change during the execution of the microprogram. Disposing of a stack (STACK) of 1-bit wide and three levels deep, we observe the following chronology: 

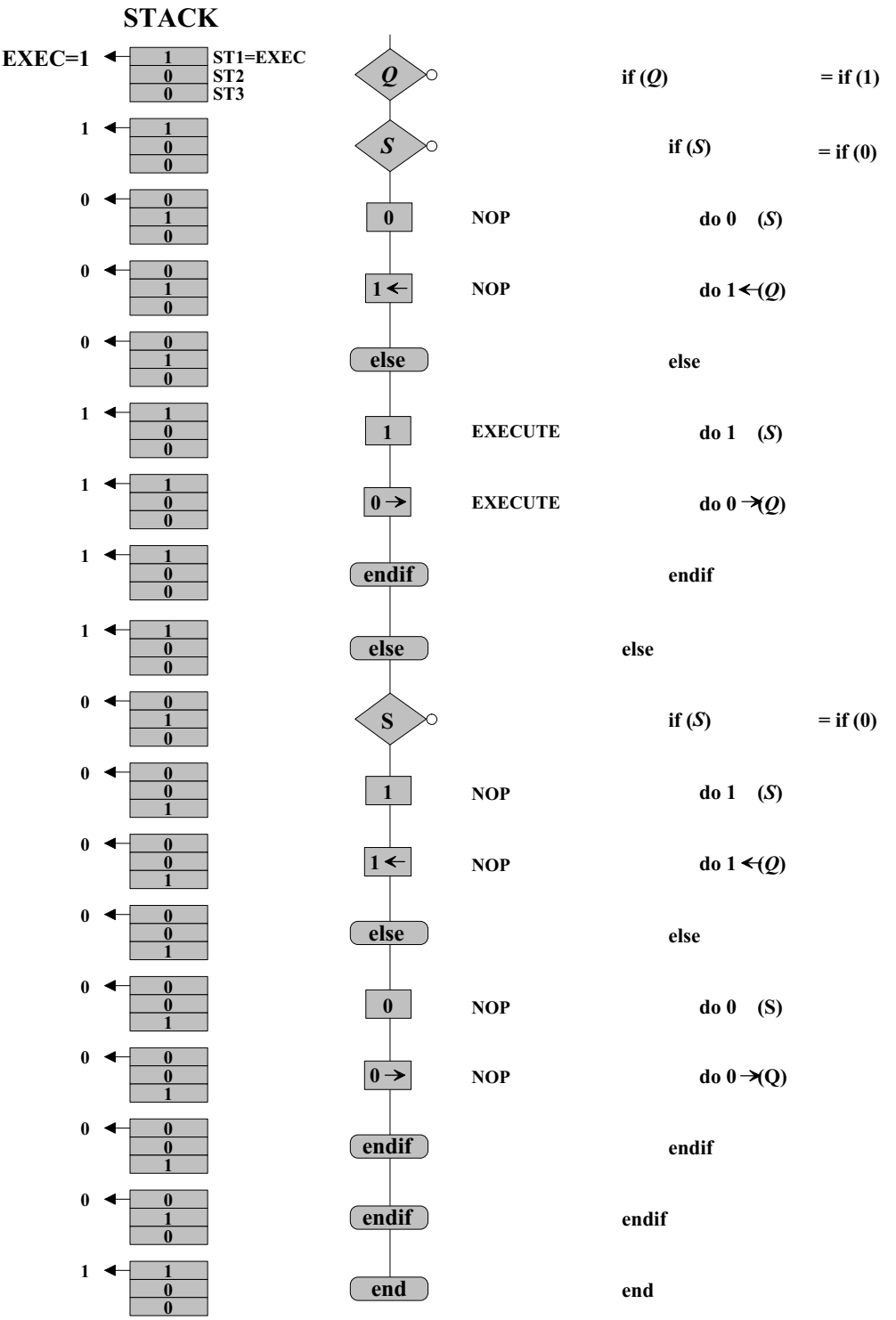

Figure 5.11: Interpretation of the program of Figures 5.4 and 5.9. The values of the test variables are $Q=1$ and $S=0$ (these values do not change during the execution of the PICOPASCAL program).

$\Rightarrow$ At the start of the program's execution, the three levels of the stack are initialized to the value $S T 3: 1=001$. The signal EXEC, which is the value at the top of the STACK, i.e., $S T 1$, is thus equal to 1 .

$\Rightarrow$ The first logic test (if $Q$ ) produces a value 1 which is placed at the top of the stack (operation PUSH). EXEC keeps the value 1.

$\Rightarrow$ The second test (if $S$ ) produces a value 0 which in turn is placed at the top of the stack (PUSH), EXEC is reset to 0 . 
$\Rightarrow$ Since the EXEC signal is 0 , the assignment do 0 , and do $1 \leftarrow$ are not executed (NOP): the stack remains in a neutral state (NOP operation) and the EXEC signal is still 0.

$\Rightarrow$ The instruction else indicates the passage from the left branch of the test (if $S$ then $P 1)$ to the right branch (else $P 2$ ). It corresponds to a COMPLEMENT operation, where the top of the STACK $(S T 1=E X E C)$ is inverted, while the content is transformed to maintain the 1-out-of-3 code. The signal EXEC is again set to 1 .

$\Rightarrow$ Since EXEC is now 1 , the assignments do 1 , and do $0 \rightarrow$ are executed.

$\Rightarrow$ The instruction endif controls the popping of the stack (POP operation). The signal EXEC keeps the value 1 to maintain the 1-out-of-3 code.

$\Rightarrow$ The execution of the program then continues as above until the final instruction end, where the stack finds again its initial state, with its first level in the state 1 $(E X E C=1)$.

\begin{tabular}{|ll|l|l|}
\multicolumn{5}{c}{ STACK6:3 } \\
\begin{tabular}{|ll|l|l|}
\hline B & A & operation & OPC \\
\hline \hline 0 & 0 & HOLD & others \\
\hline 0 & 1 & SHIFT RIGHT & endif \\
\hline 1 & 0 & SHIFT LEFT & if \\
\hline 1 & 1 & LOAD & $\Phi$ \\
\hline
\end{tabular}
\end{tabular}

\begin{tabular}{|ll|l|l|}
\multicolumn{5}{c}{ STACK2 } \\
\begin{tabular}{|ll|l|l|}
\hline B & A & operation & OPC \\
\hline \hline 0 & 0 & HOLD & others \\
\hline 0 & 1 & SHIFT RIGHT & endif \\
\hline 1 & 0 & SHIFT LEFT & else \\
\hline 1 & 1 & LOAD & if \\
\hline
\end{tabular}
\end{tabular}

\begin{tabular}{|ll|l|l|}
\multicolumn{5}{c}{ STACK1 } \\
\begin{tabular}{|ll|l|l|}
\hline B & A & operation & OPC \\
\hline \hline 0 & 0 & HOLD & others \\
\hline 0 & 1 & SHIFT RIGHT & else \\
\hline 1 & 0 & SHIFT LEFT & endif \\
\hline 1 & 1 & LOAD & if \\
\hline
\end{tabular}
\end{tabular}

$\Phi$ represents the "don't care" condition

(a)

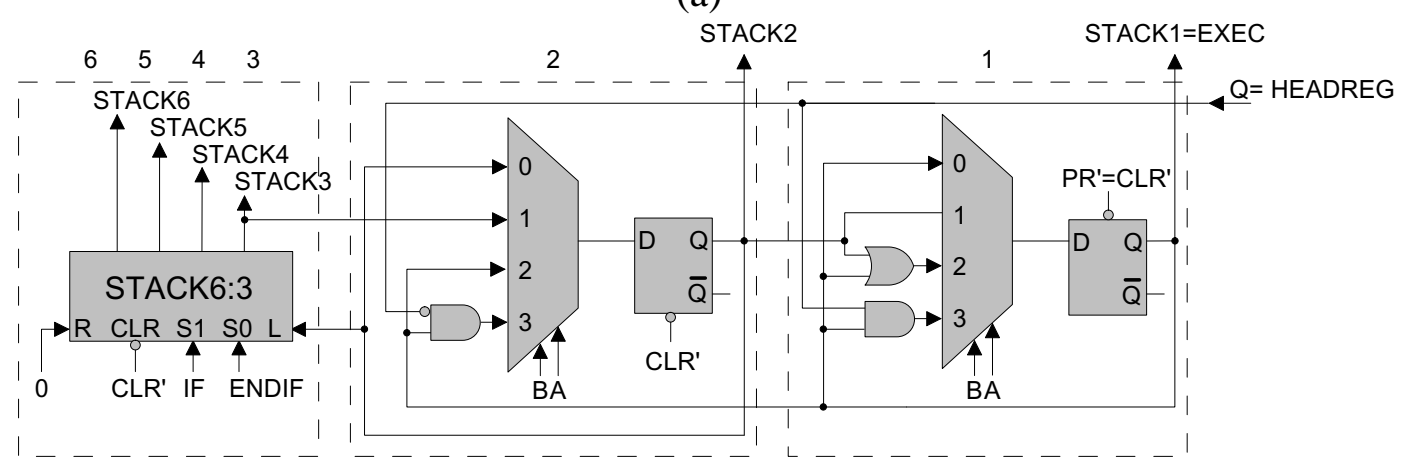

(b)

Figure 5.12: 6-level PICOPASCALINE stack. (a) Operation tables. (b) Detailed architecture.

The operation table of Figure 5.12a describes the global operation of the stack. To assure a simple initialization of the stack, we opted for a general asynchronous reset to ST3:1=001. The logic diagram of Figure $5.12 \mathrm{~b}$ describes a possible realization of the stack, according to the table of operations of Figure 5.12a. With the exception of the first and second levels, we note the iterative nature of this stack, which contains six levels 
in this implementation, is thus capable of successively testing up to six variables (six is therefore the highest number of nested tests). The global operation of the $(Q, S)$ REGISTER and of the (QL3:0,QC,QR0:3) REGISTER in Figure 5.10 is described, respectively, by the operation tables of Figures 5.13a and 5.13b.

\begin{tabular}{|c|l|c|l|c|c|c|c|}
\hline OPC & Instruc. & EXEC & \multicolumn{1}{|c|}{ OP } & R1 & R0 & L & R \\
\hline \hline 0 & NOP & $\Phi$ & HOLD & 0 & 0 & $\Phi$ & $\Phi$ \\
\hline 1 & begin & $\Phi$ & HOLD & $\mathbf{0}$ & $\mathbf{0}$ & $\Phi$ & $\Phi$ \\
\hline $\mathbf{2}$ & end & $\Phi$ & LOAD & $\mathbf{1}$ & $\mathbf{1}$ & $\Phi$ & $\Phi$ \\
\hline $\mathbf{3}$ & $\Phi$ & $\Phi$ & HOLD & $\mathbf{0}$ & $\mathbf{0}$ & $\Phi$ & $\Phi$ \\
\hline $\mathbf{4}$ & else & $\Phi$ & HOLD & $\mathbf{0}$ & $\mathbf{0}$ & $\Phi$ & $\Phi$ \\
\hline $\mathbf{5}$ & if & $\mathbf{0}$ & HOLD & $\mathbf{0}$ & $\mathbf{0}$ & $\Phi$ & $\Phi$ \\
\hline $\mathbf{6}$ & endif & $\Phi$ & HOLD & $\mathbf{0}$ & $\mathbf{0}$ & $\Phi$ & $\Phi$ \\
\hline $\mathbf{7}$ & $\Phi$ & $\Phi$ & HOLD & $\mathbf{0}$ & $\mathbf{0}$ & $\Phi$ & $\Phi$ \\
& & & & & & & \\
\hline
\end{tabular}

\begin{tabular}{|c|c|c|c|c|c|c|c|}
\hline OPC & Instruc. & EXEC & OP & R1 & R0 & $\mathbf{L}$ & $\mathbf{R}$ \\
\hline 8 & do $0 \rightarrow$ & $\begin{array}{l}\mathbf{0} \\
1\end{array}$ & $\begin{array}{l}\text { HOLD } \\
\text { SHIFT RIGHT }\end{array}$ & $\begin{array}{l}\mathbf{0} \\
\mathbf{0}\end{array}$ & $\begin{array}{l}\mathbf{0} \\
\mathbf{1}\end{array}$ & $\begin{array}{l}\Phi \\
\Phi\end{array}$ & $\begin{array}{l}\Phi \\
\mathbf{0}\end{array}$ \\
\hline 9 & do $1 \leftarrow$ & $\begin{array}{l}\mathbf{0} \\
1\end{array}$ & $\begin{array}{l}\text { HOLD } \\
\text { SHIFT RIGHT }\end{array}$ & $\begin{array}{l}\mathbf{0} \\
\mathbf{0}\end{array}$ & $\begin{array}{l}\mathbf{0} \\
1\end{array}$ & $\begin{array}{l}\Phi \\
\Phi\end{array}$ & $\Phi$ \\
\hline $\mathbf{A}$ & do 0 & $\begin{array}{l}\mathbf{0} \\
1\end{array}$ & $\begin{array}{l}\text { HOLD } \\
\text { SHIFT RIGHT }\end{array}$ & $\begin{array}{l}\mathbf{0} \\
\mathbf{0}\end{array}$ & $\begin{array}{l}\mathbf{0} \\
1\end{array}$ & $\begin{array}{l}\Phi \\
\Phi\end{array}$ & $\Phi$ \\
\hline B & do 1 & $\begin{array}{l}\mathbf{0} \\
\mathbf{1}\end{array}$ & $\begin{array}{l}\text { HOLD } \\
\text { SHIFT RIGHT }\end{array}$ & $\begin{array}{l}\mathbf{0} \\
\mathbf{0}\end{array}$ & $\begin{array}{l}\mathbf{0} \\
\mathbf{1}\end{array}$ & $\underset{\Phi}{\Phi}$ & $\Phi$ \\
\hline C & $\Phi$ & $\Phi$ & HOLD & $\mathbf{0}$ & $\mathbf{0}$ & $\Phi$ & $\Phi$ \\
\hline D & $\Phi$ & $\Phi$ & HOLD & $\mathbf{0}$ & $\mathbf{0}$ & $\Phi$ & $\Phi$ \\
\hline $\mathbf{E}$ & $\Phi$ & $\Phi$ & HOLD & 0 & $\mathbf{0}$ & $\Phi$ & $\Phi$ \\
\hline $\mathbf{F}$ & $\Phi$ & $\Phi$ & HOLD & $\mathbf{0}$ & $\mathbf{0}$ & $\Phi$ & $\Phi$ \\
\hline
\end{tabular}

$\Phi$ represents the "don't care" condition

(a)

\begin{tabular}{|c|c|c|c|c|c|c|c|}
\hline OPC & Instruc. & EXEC & OP & S1 & So & B & $\mathbf{A}$ \\
\hline 8 & do $0 \rightarrow$ & $\begin{array}{l}0 \\
1\end{array}$ & $\begin{array}{l}\text { HOLD } \\
\text { SHIFT LEFT }\end{array}$ & $\begin{array}{l}\mathbf{0} \\
1\end{array}$ & $\begin{array}{l}\mathbf{0} \\
\mathbf{0}\end{array}$ & $\begin{array}{l}0 \\
1\end{array}$ & $\begin{array}{l}\mathbf{0} \\
\mathbf{0}\end{array}$ \\
\hline 9 & do $1 \leftarrow$ & $\begin{array}{l}\mathbf{0} \\
1\end{array}$ & $\begin{array}{l}\text { HOLD } \\
\text { SHIFT RIGHT }\end{array}$ & $\begin{array}{l}\mathbf{0} \\
\mathbf{0}\end{array}$ & $\begin{array}{l}\mathbf{0} \\
1\end{array}$ & $\begin{array}{l}\mathbf{0} \\
\mathbf{0}\end{array}$ & $\begin{array}{l}\mathbf{0} \\
1\end{array}$ \\
\hline A & do 0 & $\begin{array}{l}\mathbf{0} \\
1\end{array}$ & $\begin{array}{l}\text { HOLD } \\
\text { QC }<0\end{array}$ & $\begin{array}{l}\text { 0 } \\
\text { 0 }\end{array}$ & $\begin{array}{l}\text { 0 } \\
\text { 0 }\end{array}$ & $\begin{array}{l}0 \\
1\end{array}$ & $\begin{array}{l}0 \\
1\end{array}$ \\
\hline B & do 1 & $\begin{array}{l}\mathbf{0} \\
1\end{array}$ & $\begin{array}{l}\text { HOLD } \\
\text { QC } \leftarrow 1\end{array}$ & $\begin{array}{l}\mathbf{0} \\
\mathbf{0}\end{array}$ & $\begin{array}{l}\mathbf{0} \\
\mathbf{0}\end{array}$ & $\begin{array}{l}\mathbf{0} \\
1\end{array}$ & $\begin{array}{l}\mathbf{0} \\
1\end{array}$ \\
\hline & others & $\Phi$ & HOLD & 0 & 0 & $\mathbf{0}$ & 0 \\
\hline
\end{tabular}

$\Phi$ represents the "don't care" condition

(b)

Figure 5.13: Operation tables of the PICOPASCALINE registers. (a) $(Q, S) R E G$ ISTER ('194 universal shift register); the LOAD operation, only valid for the end instruction, replaces the current value of $S$ by the current value of $Q C$. (b) (QL3:0,QC,QR0:3) REGISTER (this system consists of two '194 universal shift registers, implementing QL3:0 and QR0:3, and one D-type flip-flop implementing $Q C$ and controlled by a two-variable $(B, A)$ multiplexer).

Finally, the intrinsic limitations of the PICOPASCALINE interpreter are determined by the number and size of the registers, as well as by the number of tested variables which can be stored in the stack. 


\subsection{Detailed calculation of a universal Turing machine}

As shown in Figure 5.6, the actual implementation of our UTM architecture consists of 27 cells, where each cell contains the entire genome of the organism and, depending on its position in the array, can interpret the genome and extract and execute the gene which configures it.

The following subsections will describe in detail the calculation of the entire genome microprogram, that is, of the coordinates, the initial conditions, and the distinct genes featured by our artificial organism (Figure 5.6): the program tape genes, the stack genes, the register genes, and the data tape genes.

\subsubsection{Computing the coordinates}

The local horizontal coordinate $(X)$ of a given cell is computed as a function of the horizontal coordinate of its western neighbor $(W X)$. If we represent the coordinate $W X$ in its binary form $W X 3: 0$, the specifications of Figure 5.6 allow us to derive directly the Karnaugh map for $X$ (Figure 5.14a). A “don't care" condition $(\Phi)$ is specified for the unused values.

The use of Karnaugh maps for simplifying binary decision trees [47,48] generates a tree with nine branches (Figure 5.14b), each representing a block in the map.

The local vertical coordinate $(Y)$ of a given cell is computed as a function of the vertical coordinate of its southern neighbor $(S Y)$. Unlike the organization of the rows, which requires the placement of spare cells (for self-repair and/or extension of the tape), we wish to design a cycle on the vertical coordinate in order to allow self-replication. Such a cycle is described by the expression $Y=1 \rightarrow 2 \rightarrow 3 \rightarrow 1 \rightarrow 2 \rightarrow 3$, which we code as $Y=1 \rightarrow 2 \rightarrow 0 \rightarrow 1 \rightarrow 2 \rightarrow 0$ (Figure 5.7). The Karnaugh map of Figure 5.15a, therefore, defines $Y$ as a function of $S Y 1: 0$ and leads to the final binary decision diagram and flowchart of Figure 5.15b.

\subsubsection{Computing the initial conditions}

Computing the initial conditions, i.e., setting each cell of the cellular space to a given state according to Figure 5.6, is a part of the complete microprogram.

The methodology is roughly the same as that used above for computing the coordinates. The initial state of each cell of the cellular space (Figure 5.6) is expressed as a function of the horizontal coordinate of its western neighbor $(W X)$ and of the vertical coordinate of its southern neighbor $(S Y)$. If we represent the coordinate $W X$ in its binary form $W X 3: 0$ and $S Y$ as $S Y 1: 0$, we have to solve a problem of six variables.

In our case, most of the cells have a different initial state. As a consequence, we will 


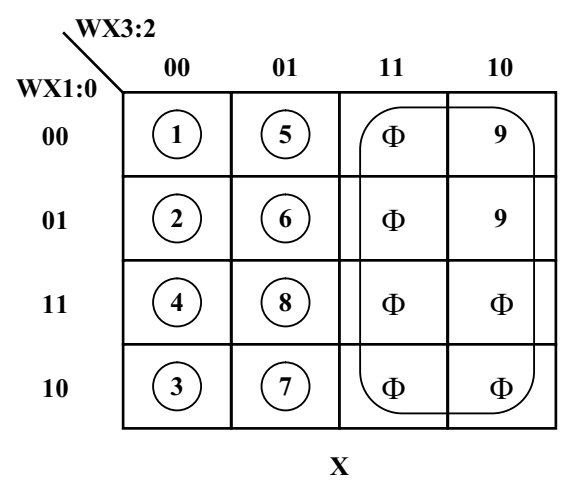

(a)
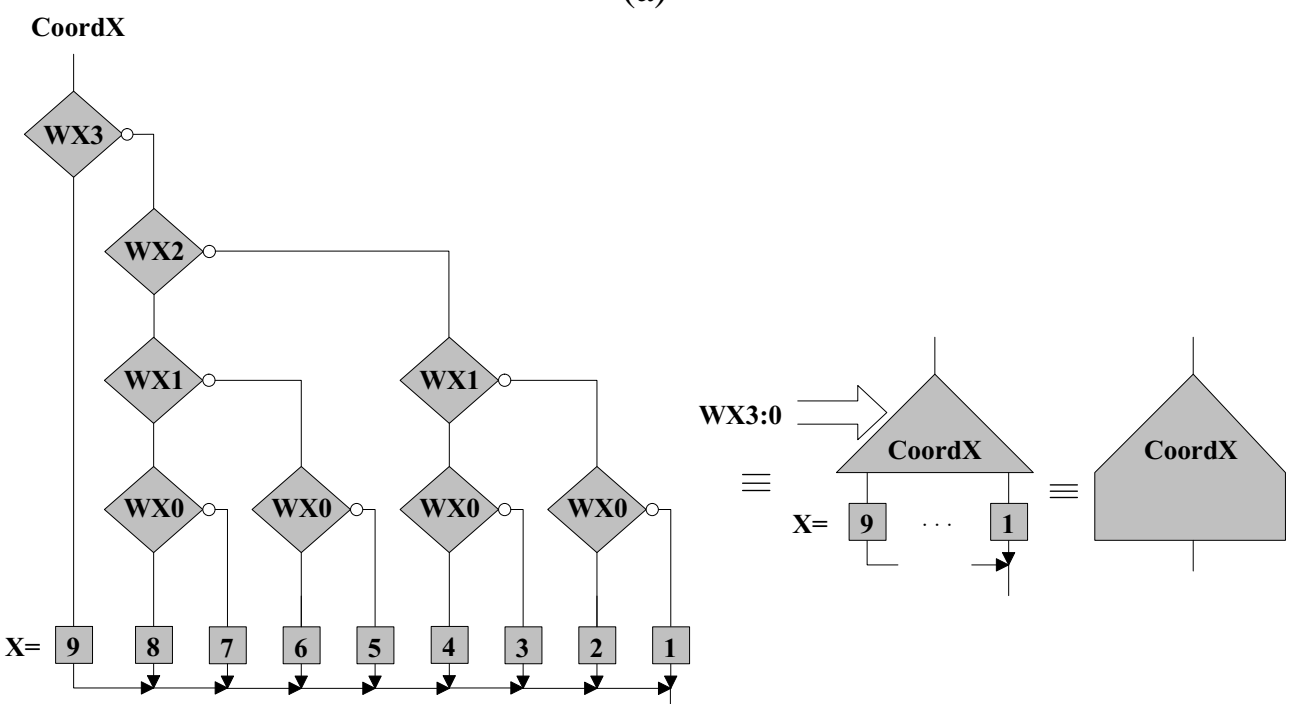

(b)

Figure 5.14: Computing the horizontal X coordinate (sub-program CoordX). (a) Karnaugh map. (b) Binary decision diagram and flowcharts.

not be able to make many simplifications to the resulting Karnaugh map, and will thus necessarily generate a large binary decision tree. A better idea is to reuse the CoordX $\mathbf{X}$ and CoordY sub-programs to access the $R E G$ register of each cell and set its initial conditions. Figure 5.16 shows the XYInitialConfig binary decision diagram and flowcharts, obtained by reusing the CoordX and CoordY sub-programs.

\subsubsection{Computing the operative genome}

From the description of Figure 5.6, we can observe that our artificial organism is composed of four main parts:

$\Rightarrow$ The program tape realizes the PICOPASCAL program tape and is implemented by the two lower rows $(Y=1,2)$ of the array. Its architecture consists of a shift register, based on three different kinds of cells. 

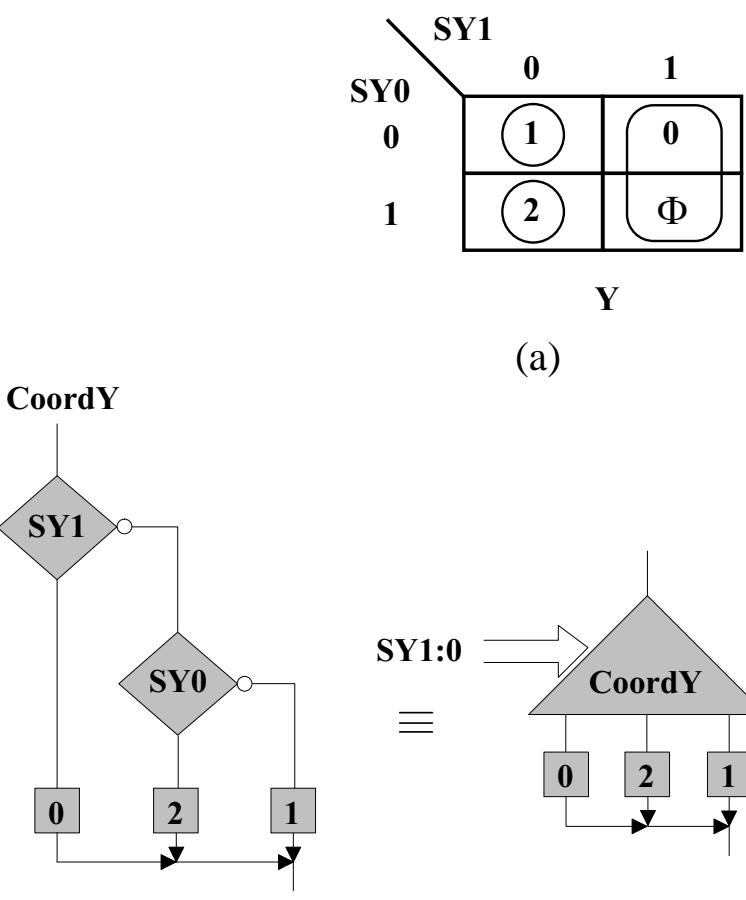

(a)

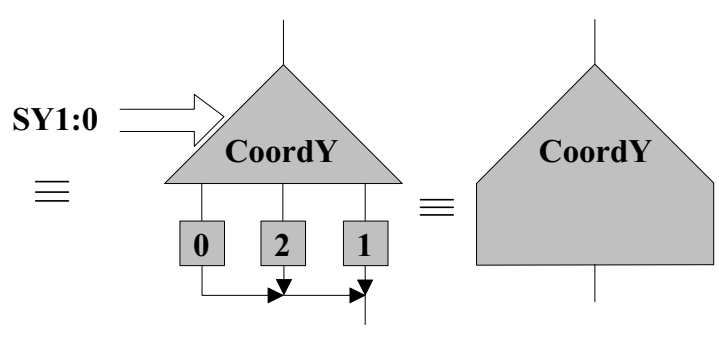

(b)

Figure 5.15: Computing the vertical $Y$ coordinate (sub-program CoordY). (a) Karnaugh map. (b) Binary decision diagram and flowcharts.

$\Rightarrow$ The stack is implemented by three cells ST3:1 $(X=1 . .3, Y=3)$. Each of these three cells is different and is described by a specific gene.

$\Rightarrow$ The register is implemented by two different cells $Q, S(X=4,5, Y=3)$ and is therefore described by two specific genes.

$\Rightarrow$ The data tape is implemented by three different cells $Q L 3: 0, Q C, Q R 0: 3(X=6 . .8$, $Y=3$ ) and is described by three specific genes.

Cellular differentiation occurs through the vertical coordinate, computed as a function of the coordinate $S Y$ of the preceding cell (the southern neighbor), and through the horizontal coordinate, computed as a function of the coordinate $W X$ of the preceding cell (the western neighbor). From Figure 5.6, we can show that the vertical coordinate $S Y$ can be used to differentiate the stack genes, the register genes, and the data tape genes $(S Y=2)$ from the program tape genes $(S Y=1,0)$.

The Karnaugh map of Figure 5.19a, derived from Figure 5.6, defines the placement of the stack (ST3:1), register $(Q, S)$, and data tape (QL3:0, QC, QR0:3) genes into the cellular space. The sub-tree contained by the leftmost dashed square of Figure $5.19 \mathrm{~b}$ implements this Karnaugh map. To find the different genes of the program tape, we need to analyze in more detail its particular architecture. 


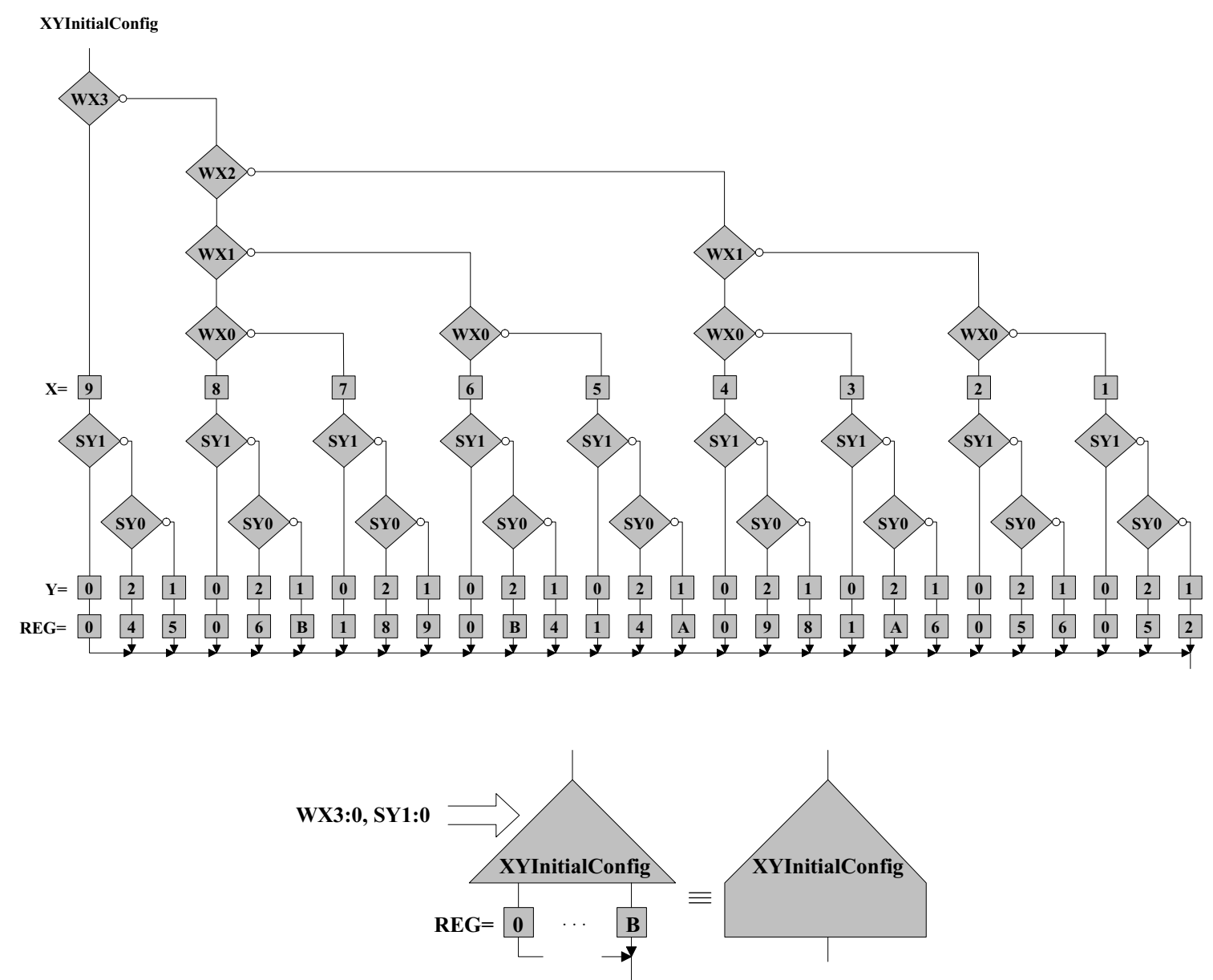

Figure 5.16: XYInitialConfig binary decision diagram and flowchart resulting from reusing the CoordX and $\mathbf{C o o r d} \mathbf{Y}$ sub-programs to set the initial conditions.

Each cell of our program tape $(Y=1,2)$ implements one PICOPASCAL instruction (stored in the four-bit $R E G$ register) and at each program step every instruction has to be shifted anticlockwise. From Figure 5.17, which shows the routing path established between each cell to transfer the instruction to its neighbor, we have to consider three different situations, which will be used to identify the position of the three specific genes:

$\Rightarrow$ the cells at coordinates $(W X=0 . .7, S Y=1)$ will receive, decode and store (in the $R E G$ register) the instruction of the east neighbor through the input bus EI3:0;

$\Rightarrow$ the cells at coordinates ( $W X=8,9, S Y=1$ ) will receive, decode and store (in the $R E G$ register) the instruction of the south neighbor through the input bus SI3:0;

$\Rightarrow$ the cells at coordinates $(W X=0 . .9, S Y=0)$ will receive, decode and store (in the $R E G$ register) the instruction of the north neighbor through the input bus NI3:0. 


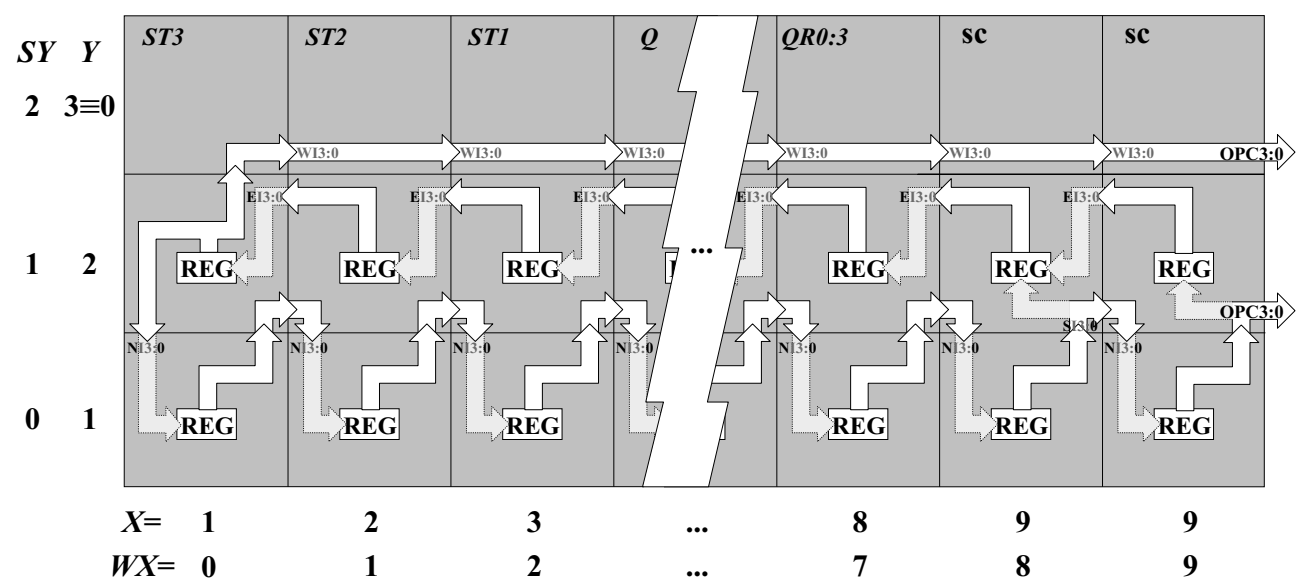

Figure 5.17: Routing path established between the cells of the program tape $(S Y=0,1)$ to implement the anticlockwise shift of the PICOPASCAL program.

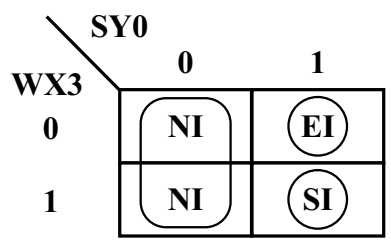

SOURCE

(a)
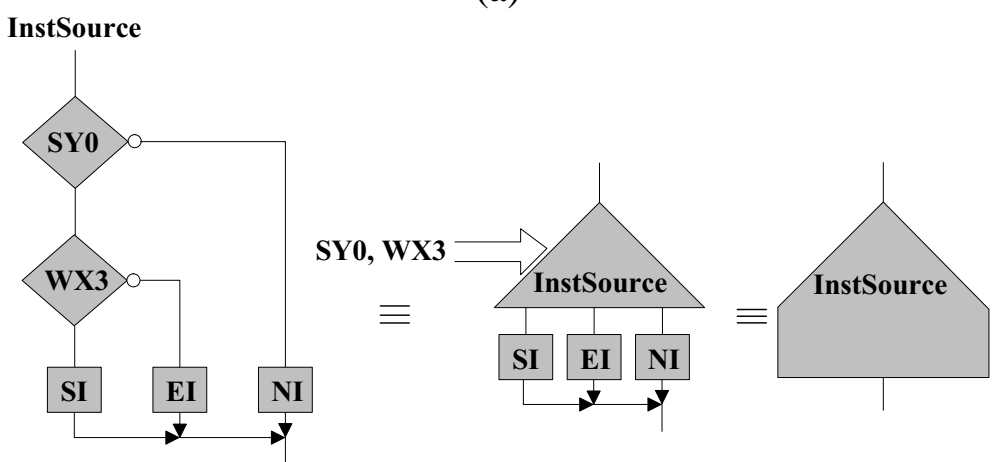

(b)

Figure 5.18: InstSource program for the differentiation of the tape genes. (a) Karnaugh map. (b) Binary decision diagram and flowcharts.

The functionality of each group of cells of the program tape can be expressed as a function of the horizontal coordinate (WX3) and of the vertical coordinate $(S Y O)$. We therefore have to solve a problem of two variables, as shown by Karnaugh map of Figure $5.18 \mathrm{a}$. Figure $5.18 \mathrm{~b}$ shows the resulting binary decision diagram.

By joining the binary decision diagram of Figure 5.18b (InstSource) and the binary decision diagram derived from the Karnaugh map of Figure 5.19a (sub-tree contained by the leftmost dashed square of Figure 5.19b), we can generate the binary decision diagram 


\begin{tabular}{|c|c|c|c|c|}
\hline & 00 & 01 & 11 & 10 \\
\hline 00 & ST3) & $\mathbf{S}$ & $\Phi$ & $\Phi$ \\
\hline 01 & $\mathrm{ST} 2)$ & $\mathbf{Q L}$ & $\Phi$ & $\Phi$ \\
\hline 11 & $\mathbf{Q}$ & QR & $\Phi$ & $\Phi$ \\
\hline 10 & ST1) & QC & $\Phi$ & $\Phi$ \\
\hline
\end{tabular}

(a)

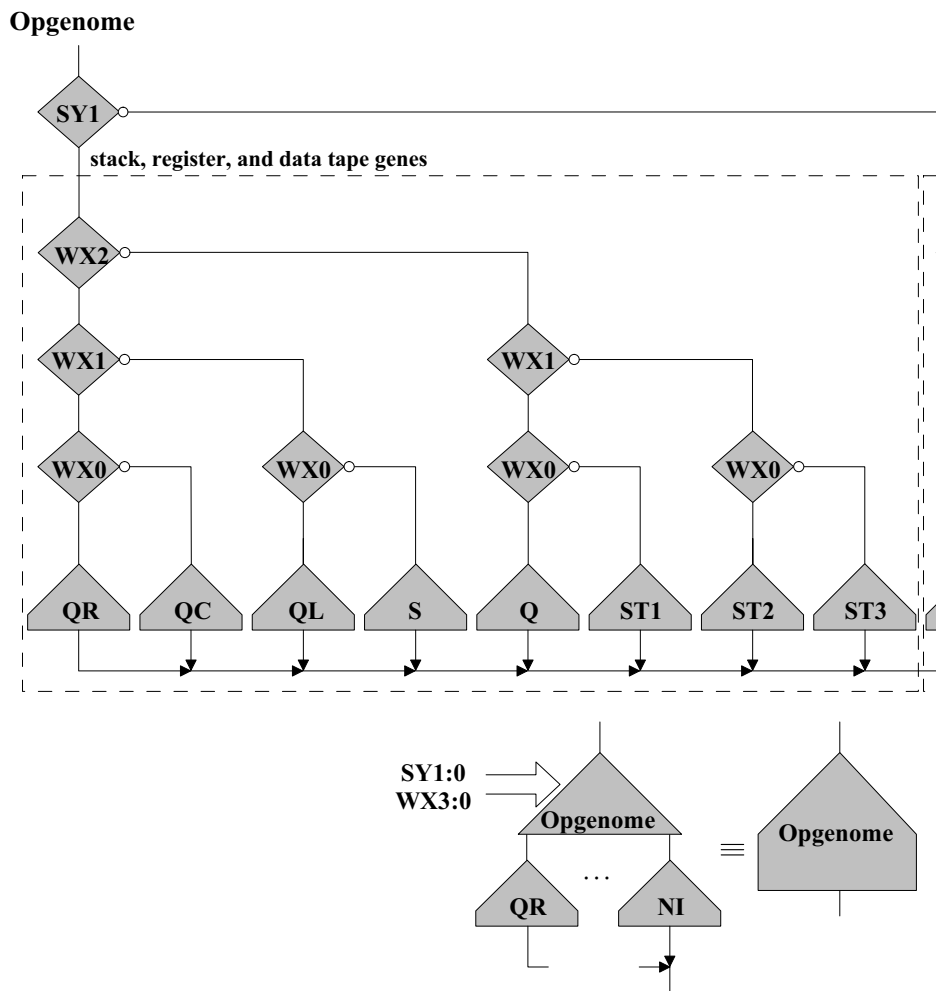

(b)

Figure 5.19: Computing the genome's operational part (sub-program Opgenome). (a) Karnaugh map for stack (ST3:1), register $(Q, S)$, and data tape genes (QL3:0, QC, QR0:3). (b) Binary decision diagram and flowchart of the genome's operational part.

and flowchart of Figure 5.19b describing the complete operational part of the genome of our UTM implementation (Opgenome).

\subsubsection{Computing the program tape genes}

Figure $5.6(Y=1,2)$ shows that the program tape, in our implementation, is composed of 18 cells and features three different genes $(E I, S I$, and $N I)$. Since the begin instruction is 
not executed and the NOP instruction is not used, these cells have to deal with a program composed of eight different kinds of PICOPASCAL instructions (Figure 5.9a).

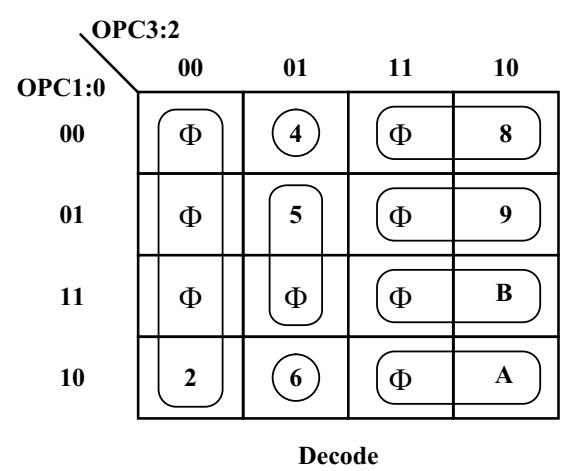

(a)

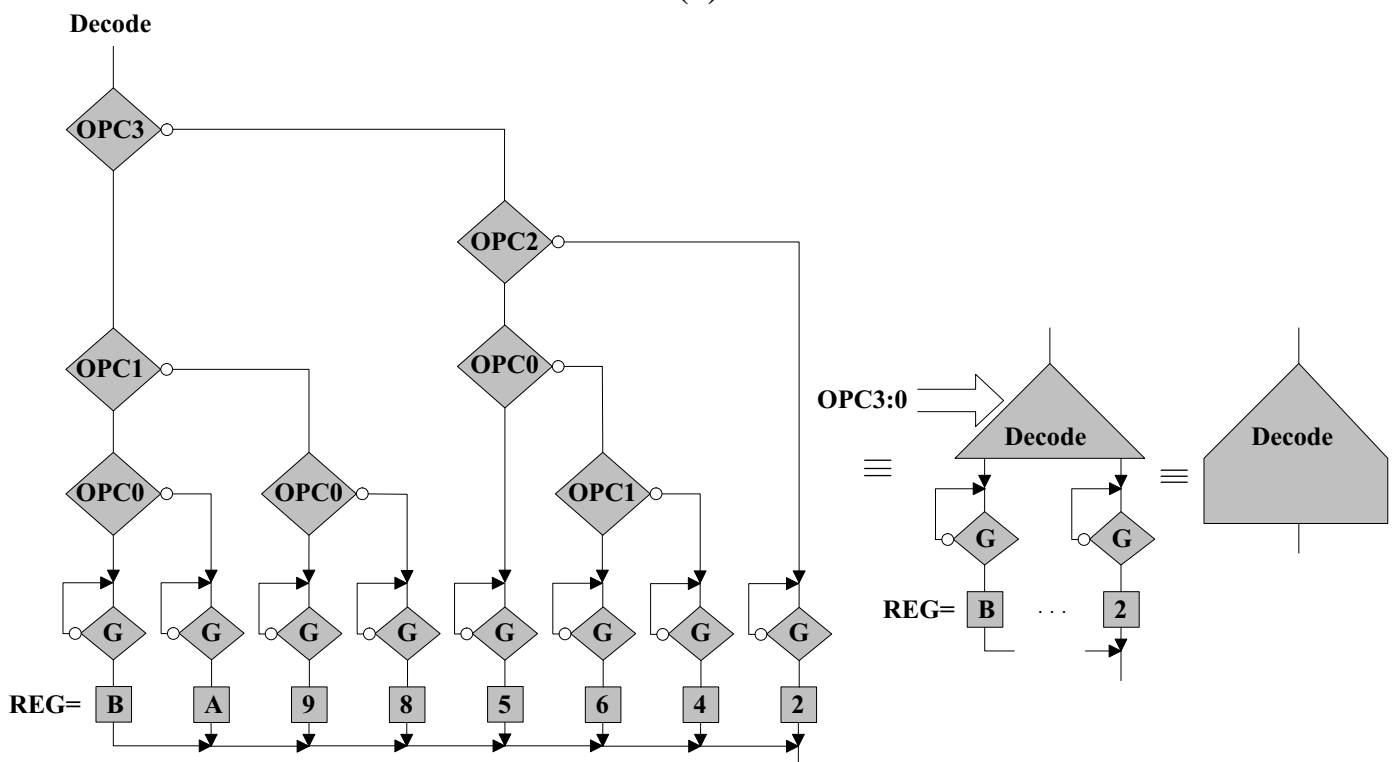

(b)

Figure 5.20: Generic decoding of the instruction to be assigned to REG. (a) Karnaugh map. (b) General binary decision diagram and flowcharts (Decode).

Therefore, each cell has to decode the instruction coming from its neighbor (east, south, or north) and store it in the REG register. The Karnaugh map of Figure 5.20a shows the binary coding proposed for the eight PICOPASCAL instructions stored in the program tape. Figure $5.20 \mathrm{~b}$ shows the generic binary decision diagram that we use to decode the instruction to be assigned to the register $R E G$. In consequence, we can implement the three genes $E I, S I$, and $N I$, of the program tape by decoding the instructions coming from the east, south, and north neighbors respectively, that is, by replacing OPC3:0 (Figure 5.20b) by EI3:0, SI3:0, and NI3:0 respectively.

Figure 5.21 shows the final flowcharts for the genes EI, SI, and NI, which are instances of the Decode sub-program. To assure the synchronization of all the registers, 
tests are performed throughout the half-period when $G=0$, but no assignment is made until the rising edge of $G(G=0 \rightarrow 1)$, when all the registers $R E G$ are updated simultaneously.

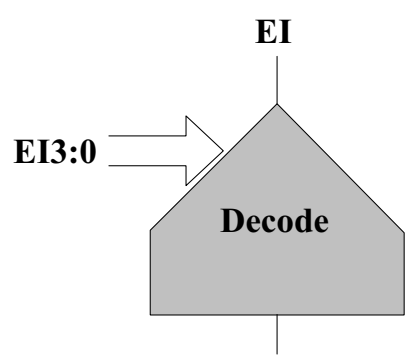

(a)

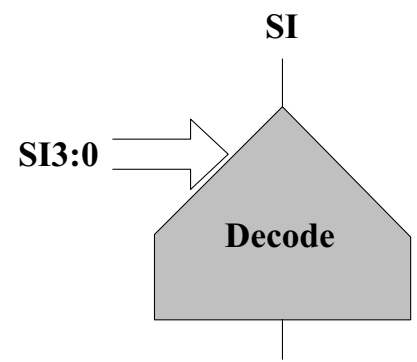

(b)

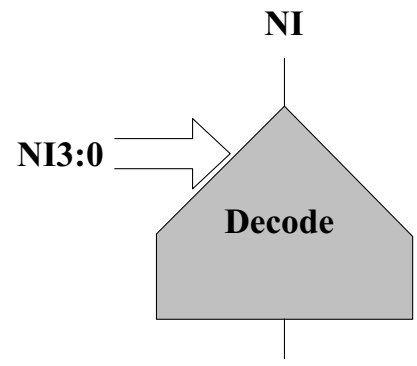

(c)

Figure 5.21: Final flowcharts for the genes composing the program tape. (a) EI gene computation. (b) SI gene computation. (c) NI gene computation.

\subsubsection{Computing the stack genes}

As shown in Figure 5.6, the stack part of our cellular UTM implementation is composed of the three cells ST3:1 $(X=1 . .3, Y=3)$, each featuring a different gene (ST1, ST2, and ST3). The Embryonics implementation of the STACK part of our artificial organism (the PICOPASCALINE stack) has to reproduce the behavior described by the stack operation tables in Figure 5.12a and by the stack architecture presented in Figure 5.12b. From the tables we obtain the information to build the Karnaugh maps decoding the PICOPASCAL instructions related with each stack gene (ST1, ST2, ST3), and from the architecture we obtain the logic part and the corresponding control signals.

\section{Computing the $S T 1$ gene}

To implement the ST1 gene we need to decode the PICOPASCAL instructions related to the $S T 1$ cell, that is, the instructions if, endif, else, and OI (other instructions) described by the OPC column of the STACK1 table in Figure 5.12a. These instructions can be decoded by the Karnaugh map of Figure 5.22a, which is implemented by the sub-tree contained by the dashed square of Figure 5.22b. As the PICOPASCAL instruction being decoded comes into the STI cell through the WI3:0 bus (Figure 5.17), we use WI as the test variable for the Karnaugh map.

We then need to implement the logic part of the $S T 1$ gene, contained by the rightmost dashed square (level 1) of Figure 5.12b. From this figure we identify three logic signals:

$\Rightarrow$ STACK1 or EXEC signal, which will correspond in our implementation to the bit $R E G O$ of the $S T 1$ cell; 


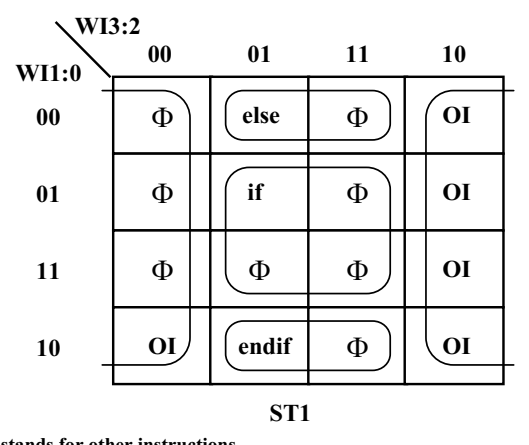

(a)
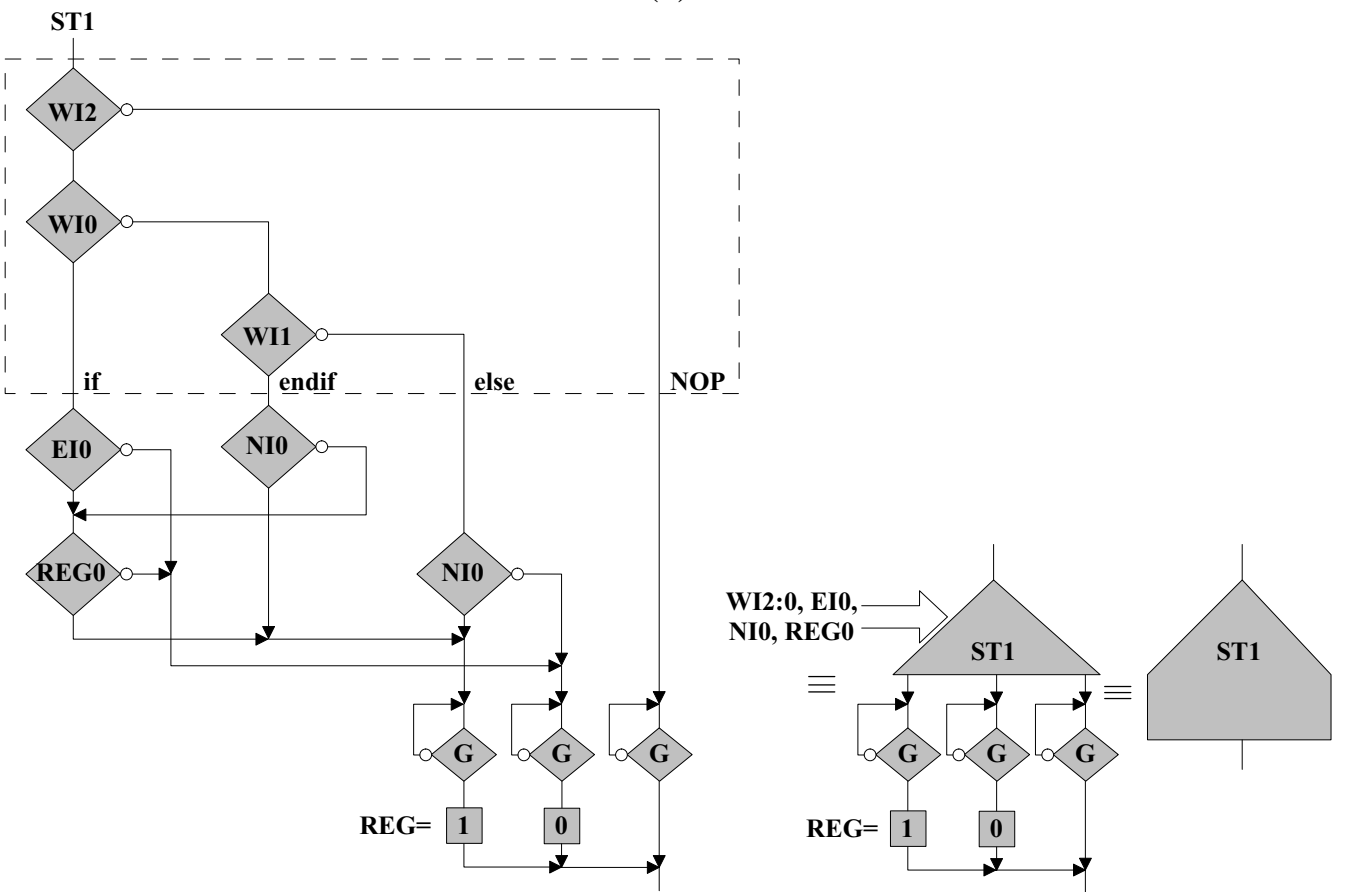

(b)

Figure 5.22: ST1 gene computation. (a) Karnaugh map decoding the instructions in the OPC column in the STACK1 table in Figure 5.12a. (b) Binary decision diagram and flowchart of the gene ST1.

$\Rightarrow$ STACK2, which will correspond in our implementation to the output of the STACK2 stage (level 2) and comes into the STI cell through the NIO input;

$\Rightarrow H E A D R E G$, which will correspond in our implementation to the output of the $Q$ cell and comes into the $S T 1$ cell through the EIO input.

The $S T 1$ gene is described by the binary decision diagram of Figure 5.22b, and the implementation of the logic part is realized by the portion of the binary decision diagram outside of the dashed square in the same figure. Figure 5.34 shows the details of the different routing paths for the stack cells. 


\section{Computing the $S T 2$ gene}

The Karnaugh map of Figure 5.23a decodes the PICOPASCAL instructions related to the $S T 2$ cell, that is, the instructions if, endif, else, and OI (other instructions) described by the OPC column of the STACK2 table in Figure 5.12a. This map is implemented by the sub-tree contained by the dashed square of Figure 5.23b. As the PICOPASCAL instruction being decoded comes into the ST2 cell through the WI3:0 bus (Figure 5.17), we use $W I$ as the test variable for the Karnaugh map.

\begin{tabular}{|c|c|c|c|c|}
\hline WI1:0 & 00 & 01 & 11 & 10 \\
\hline 00 & $\Phi$ & (else & $\Phi$ & OI \\
\hline 01 & $\Phi$ & if & $\Phi$ & OI \\
\hline 11 & $\Phi$ & $\Phi$ & $\Phi$ & OI \\
\hline 10 & OI & (endif & $\Phi$ & OI \\
\hline
\end{tabular}

(a)

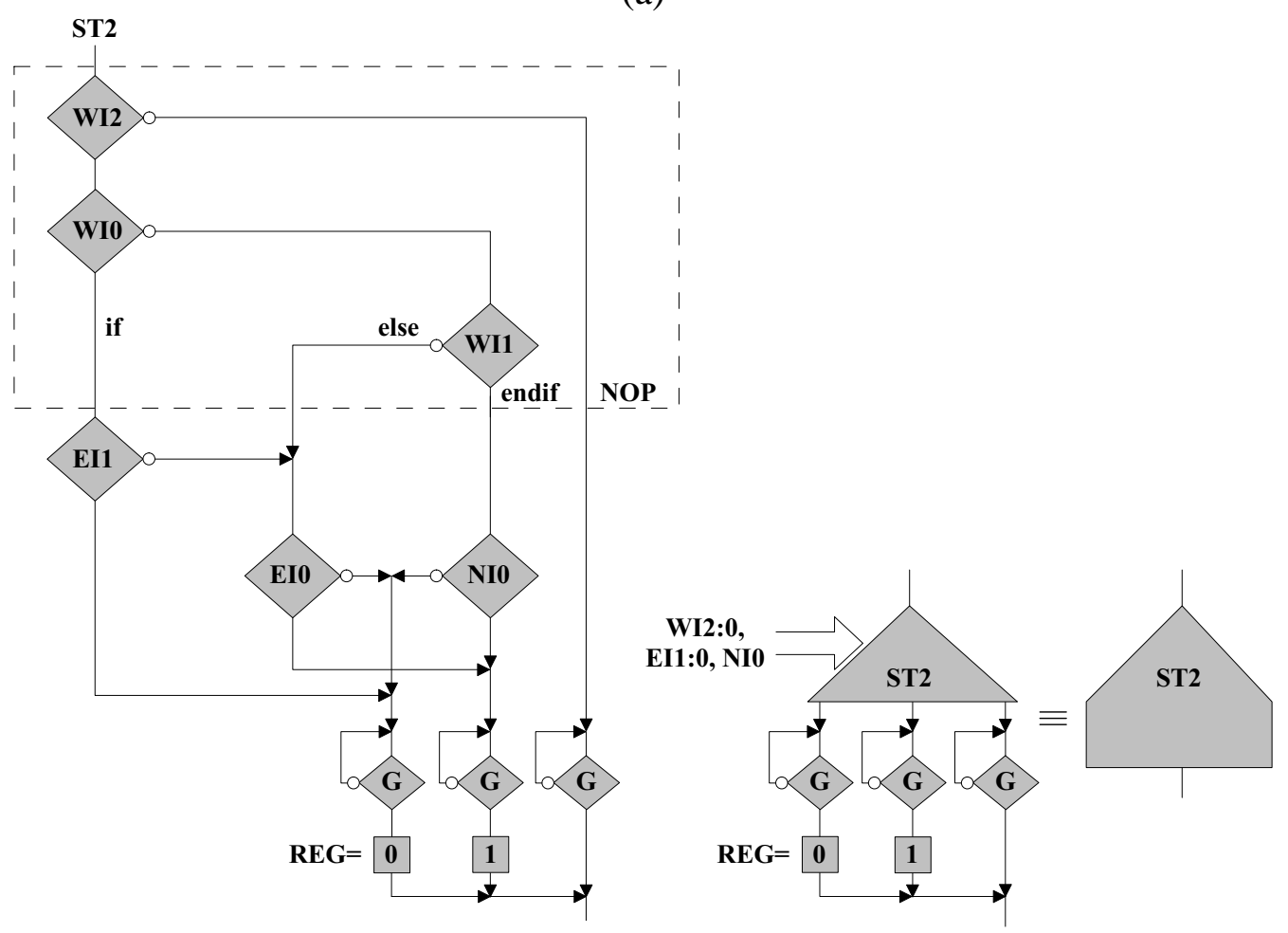

(b)

Figure 5.23: ST2 gene computation. (a) Karnaugh map. (b) Binary decision diagram and flowcharts.

We then need to implement the logic part of the ST2 gene, contained by the middle dashed square (level 2) of Figure 5.12b. From this figure we identify the following four 
logic signals:

$\Rightarrow$ STACK2, which will correspond in our implementation to the bit REGO of the ST2 cell;

$\Rightarrow$ STACK1 or EXEC signal, which will correspond in our implementation to the output of the STACK1 stage (level 1) and comes into the $S T 2$ cell through the EIO input;

$\Rightarrow$ STACK3, which will correspond in our implementation to the output of the STACK3 stage (level 3) and comes into the ST2 cell through the NIO input;

$\Rightarrow$ HEADREG, which will correspond in our implementation to the output of the $Q$ cell and comes into the $S T 2$ cell through the EII input.

The $S T 2$ gene is described by the binary decision diagram of Figure 5.23b. The implementation of the logic part is realized by the portion of the binary decision diagram outside of the dashed square of the same figure. Figure 5.34 shows the details of the different routing paths for the stack cells.

\section{Computing the $S T 3$ gene}

The Karnaugh map of Figure 5.24a decodes the PICOPASCAL instructions related to the ST3 cell, that is, the instructions if, endif, and OI (other instructions) described by the OPC column of the STACK3 table in Figure 5.12a. This map is implemented by the subtree contained by the dashed square of Figure 5.24b. As the PICOPASCAL instruction being decoded comes into the ST3 cell through the SI3:0 bus (Figure 5.17), we use SI as the test variable for the Karnaugh map.

We then need to implement the logic part of the ST3 gene, contained by the leftmost dashed square (level 3) of Figure 5.12b. From this figure we identify two logic signals:

$\Rightarrow S T A C K 3$, which will correspond in our implementation to the bit REGO of the ST3 cell;

$\Rightarrow$ STACK2, which will correspond in our implementation to the output of the STACK2 stage (level 2) and comes into the ST2 cell through the EIO input;

The $S T 3$ gene is described by the binary decision diagram of Figure 5.24b. The implementation of the logic part is realized by the portion of the binary decision diagram outside of the dashed square of the same figure. Figure 5.34 shows the details of the different routing paths for the stack cells. 


\begin{tabular}{|c|c|c|c|c|}
\hline SI1:0 & 00 & 01 & 11 & 10 \\
\hline 00 & $\Phi$ & OI & $\Phi$ & OI \\
\hline 01 & $\Phi$ & if & $\Phi$ & OI \\
\hline 11 & $\Phi$ & $\Phi$ & $\Phi$ & OI \\
\hline 10 & OI & (endif & $\Phi$ & OI \\
\hline
\end{tabular}

(a)
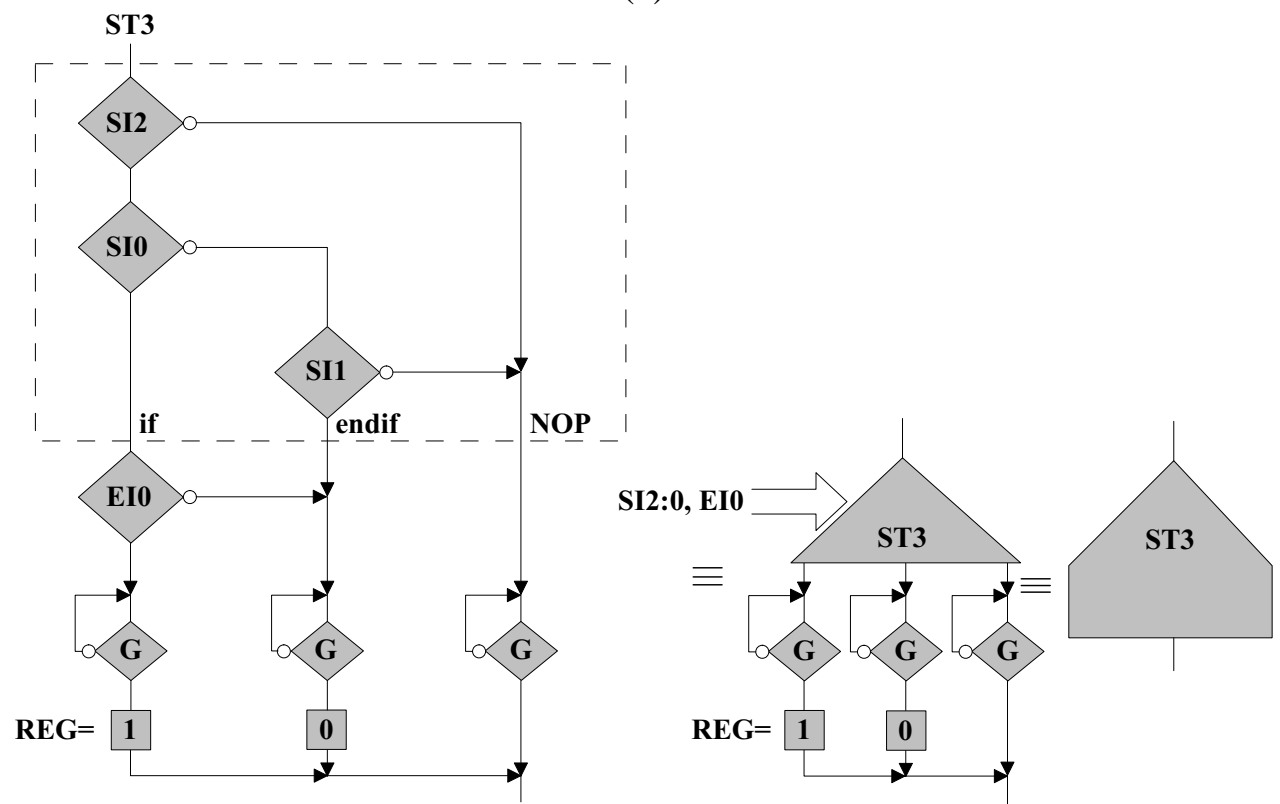

(b)

Figure 5.24: ST3 gene computation. (a) Karnaugh map. (b) Binary decision diagram and flowcharts.

\subsubsection{Computing the $(Q, S)$ REGISTER genes}

To implement the behavior of the PICOPASCALINE register with the MICTREE cells $Q$ and $S$, we need to analyze the operation table of Figure 5.13 and the register logic diagram of Figure 5.10. From the former we can obtain the Karnaugh maps used to decode the related PICOPASCAL instructions, and with the latter we can implement the register logic.

\section{Computing the $Q$ gene}

The Karnaugh map of Figure 5.25a decodes the PICOPASCAL instructions related to gene $Q$. From this figure we can observe which block (set of instructions) corresponds to the register operations SHIFT LEFT, SHIFT RIGHT, and HOLD, assuming that EXEC $=1$. 


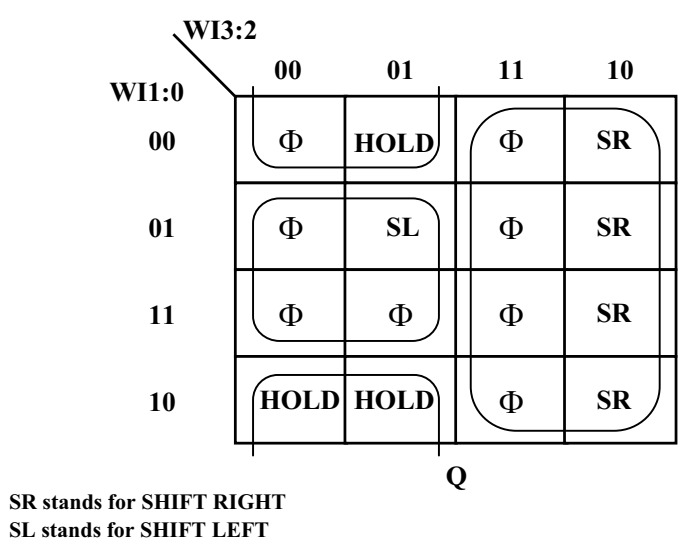

(a)

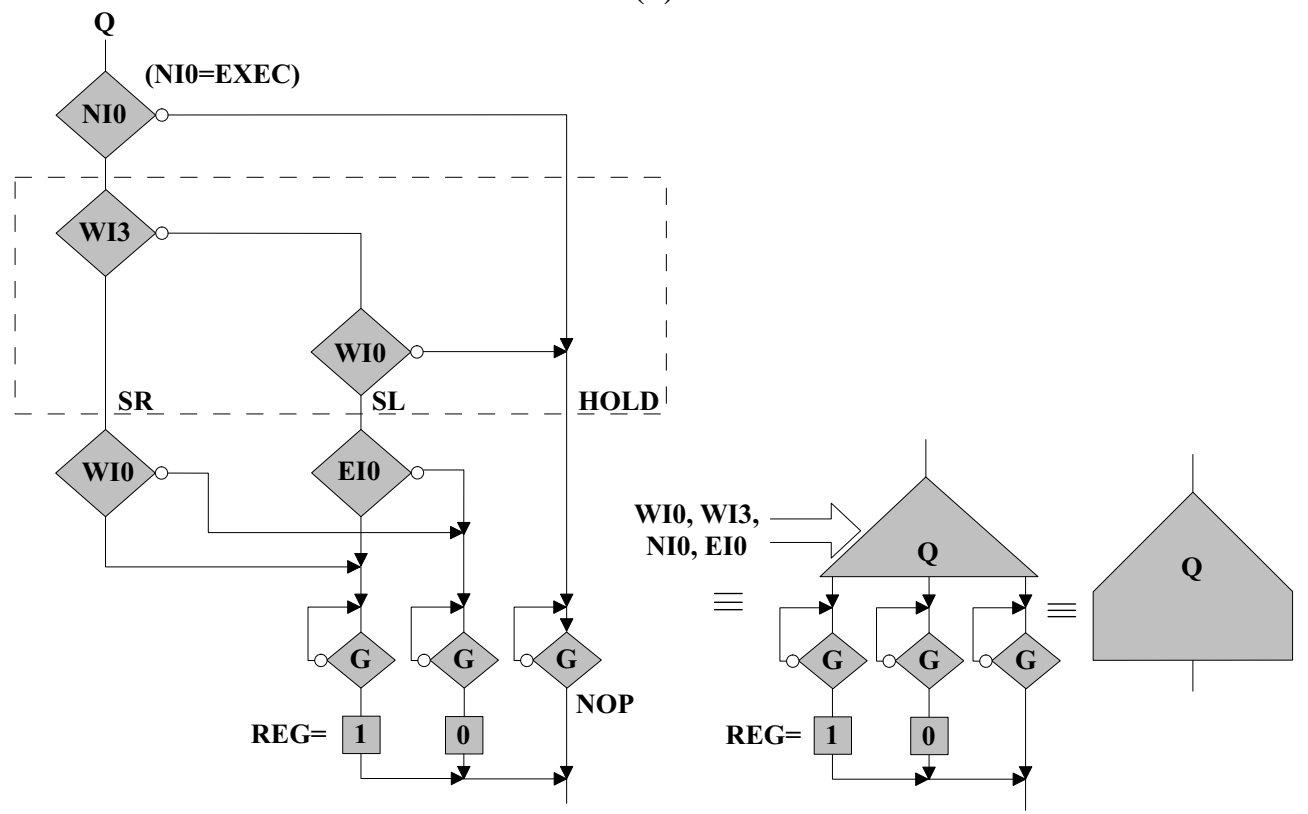

(b)

Figure 5.25: $Q$ gene computation. (a) Karnaugh map decoding the instructions in column OPC in the operation table of Figure 5.13. (b) Binary decision diagram and flowcharts.

The binary decision diagram of Figure 5.25b implements the complete gene $Q$. The logic part is realized by the portion of the binary decision diagram outside of the dashed square into the same figure, while the instruction-decoding part is realized by the sub-tree inside the dashed square. The first test in the tree $(N I O)$ corresponds to the test of the signal $E X E C$, which indicates if the assignment instruction will be executed or not. The EXEC signal comes from the left neighbor cell (ST1) and arrives through the NIO input. The last test on the left (WIO) is used to determine if the register $R E G$ of the cell $Q$ will take the value 1 (do $1 \leftarrow$, do 1 ) or 0 (do $0 \rightarrow$, do 0 ), as specified in Figure 5.13, thus implementing the shift right operation. Finally, the last test on the right (EIO) is used to assign to the register $R E G$ of the cell the value ( 0 or 1$)$ coming from the right neighbor cell $(S)$ through the EIO input, thus implementing the shift left operation. 


\section{Computing the $S$ gene}

The Karnaugh map of Figure 5.26a decodes the PICOPASCAL instructions related to gene $S$. From this figure we can observe which block (set of instructions) corresponds to the register operations (SHIFT LEFT, SHIFT RIGHT, LOAD, and HOLD) assuming that $E X E C=1$.

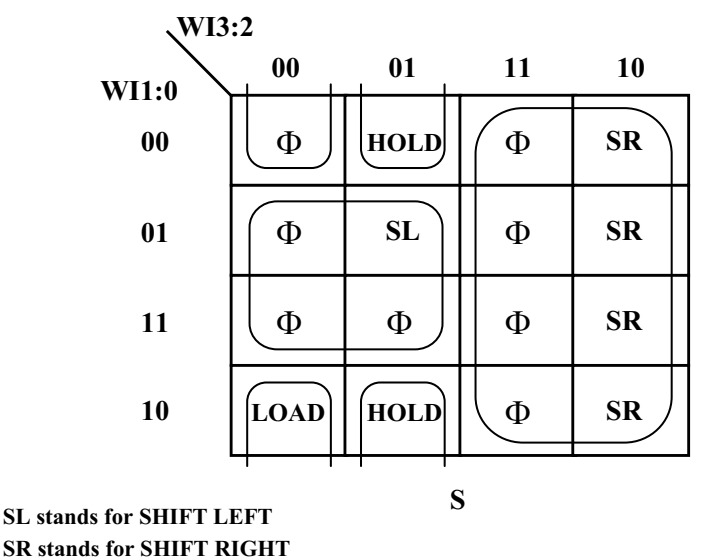

(a)
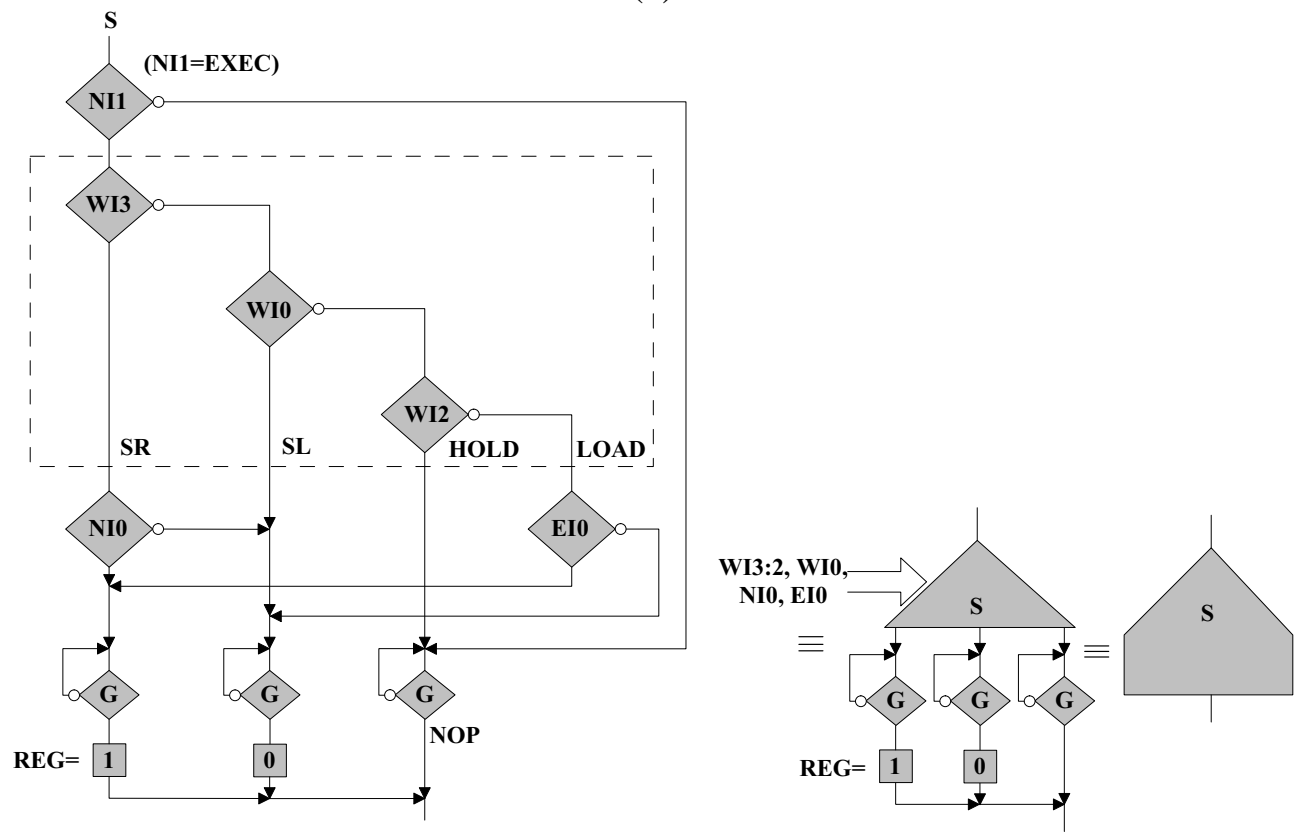

(b)

Figure 5.26: $S$ gene computation. (a) Karnaugh map decoding the instructions in column OPC in the operation table of Figure 5.13. (b) Binary decision diagram and flowcharts.

The binary decision diagram of Figure 5.26b implements the complete gene $S$. The logic part is realized by the portion of the binary decision diagram outside of the dashed square into the same figure, while the instruction-decoding part is realized by the sub-tree inside the dashed square. The first test in the tree $(N I O)$ corresponds to the test of the 
signal $E X E C$, which indicates if the assignment instruction will be executed or not. The $E X E C$ signal comes from the left neighbor cell $(Q)$ and arrives through the NII input. The last test on the left (NIO) is used to determin if the register $R E G$ of the cell $S$ will take the value ( 0 or 1$)$ coming from the left neighbor cell $(Q)$ and arriving thought the NIO input, thus implementing the shift right operation. The shift left operation is implemented by assigning the value 0 to the register $R E G$ of the cell. Finally, the last test on the right (EIO) is used to assign to the cell's register REG the value ( 0 or 1$)$ coming from the right neighbor cell $(Q C)$ through the EIO input, thus implementing the LOAD operation, allowing a new value to be stored in the $(Q, S)$ REGISTER from the central cell $Q C$ of the data tape, i.e., the $(Q L 3: 0, Q C, Q R 0: 3)$ REGISTER.

\subsubsection{Computing the (QL3:0, $Q C, Q R 0: 3)$ REGISTER genes}

In our architecture, the data tape is implemented by three cells $(W X=5 . .7, S Y=2)$, each featuring a different gene $(Q L, Q C$, and $Q R)$. These cells store the 9 bits $Q L 3: 0, Q C$, $Q R 0: 3$ (Figure 5.6) where $Q L 3: 0$ are the four squares to the left of the central square $Q C$, and $Q R 0: 3$ are the four squares to the right of $Q C$.

The data tape must be able to shift right, left, or hold (to simulate the behavior of a mobile head). Thus our data tape is basically a shift register and the direction of the shift is determined by the instruction being executed. Thus, each cell (implementing a particular gene) has to decode the current instruction before carrying out an action.

\section{Computing the $Q L$ gene}

The Karnaugh map of Figure 5.27a decodes the PICOPASCAL instructions related to the implementation of the $Q L$ gene, that is, do $0 \rightarrow$, which indicates that the data tape has to move left, and do $1 \leftarrow$, which indicates that the data tape has to move right (otherwise it should not move, as shown in Figure 5.13b).

The first test in the tree (NII) of Figure 5.27b corresponds to the test of the signal EXEC, which indicates if the assignment instruction will be executed or not. The rest of the tree (inside the dashed square) implements the Karnaugh map of Figure 5.27a. After decoding the instruction, we need to implement the shift-left and the shift-right movements of the $Q L 3: 0$ cell.

To shift right the bits of the register (REG) of the $Q L 3: 0$ cell, we need to know the value of this register. The Karnaugh map of Figure 5.28a decodes the value in the cell register and indicates the new value to be assigned to it, that is, the value after the shift right. Thus, if the current value is $R E G 3: 0=0110$, the next value will be 0011 . Note that we assume that the bit to the left of the $Q L 3: 0$ cell has the value 0. Finally, the Karnaugh map of Figure 5.28a is implemented by the binary decision diagram of Figure 5.28b.

To shift left the bits of the register (REG) of the $Q L 3: 0$ cell, we need to know the value of this register. The Karnaugh map of Figure 5.29a decodes the value in the cell register and indicates the new value to be assigned to it, that is, the value after the shift 


\begin{tabular}{|c|c|c|c|c|}
\hline WI1:0 & 00 & 01 & 11 & 10 \\
\hline 00 & $\Phi$ & HOLD & $\Phi$ & SL \\
\hline 01 & $\Phi$ & HOLD & $\Phi$ & SR \\
\hline 11 & $\Phi$ & $\Phi$ & $\Phi$ & HOLD \\
\hline 10 & HOLD & HOLD & $\Phi$ & HOLD \\
\hline
\end{tabular}

SR stands for SHIFT RIGHT

QL

SL stands for SHIFT LEFT

(a)
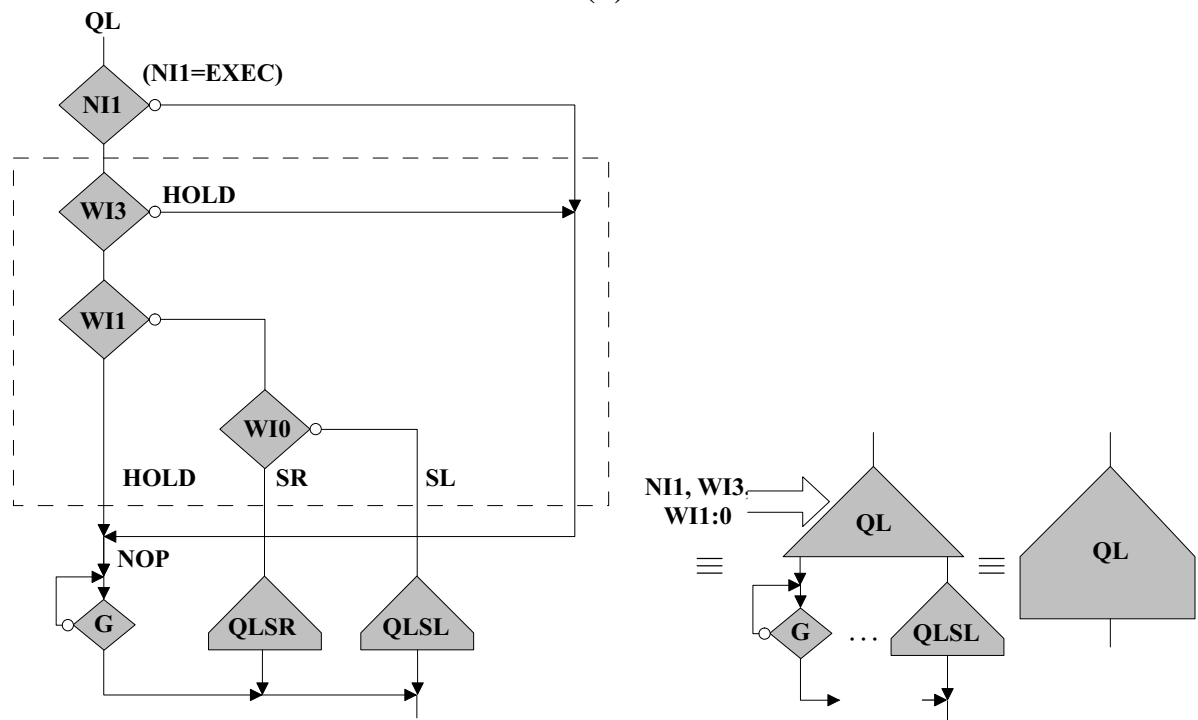

(b)

Figure 5.27: QL gene computation. (a) Karnaugh map decoding the instructions related to the cell implementing $Q L$ gene. (b) Binary decision diagram and flowcharts.

left. Thus, if the current value is $R E G 3: 0=0110$, the next value will be $110 Q C$. Note that the rightmost bit is replaced by the bit contained in the register $(R E G)$ of the $Q C$ cell. Finally, the Karnaugh map of Figure 5.29a is implemented by the binary decision diagram of Figure 5.29b.

\section{Computing the $Q R$ gene}

The Karnaugh map decoding the PICOPASCAL instructions related to the implementation of $Q R$ gene, that is, do $0 \rightarrow$, do $1 \leftarrow$, is the same as that shown in Figure 5.27a.

The first test in the tree (NII) of Figure 5.30 corresponds to the test of the signal $E X E C$, which indicates if the assignment instruction will be executed or not. The rest 


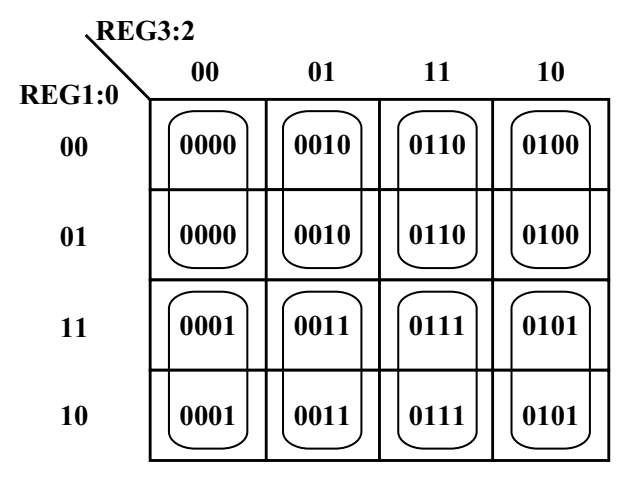

REG+

(a)
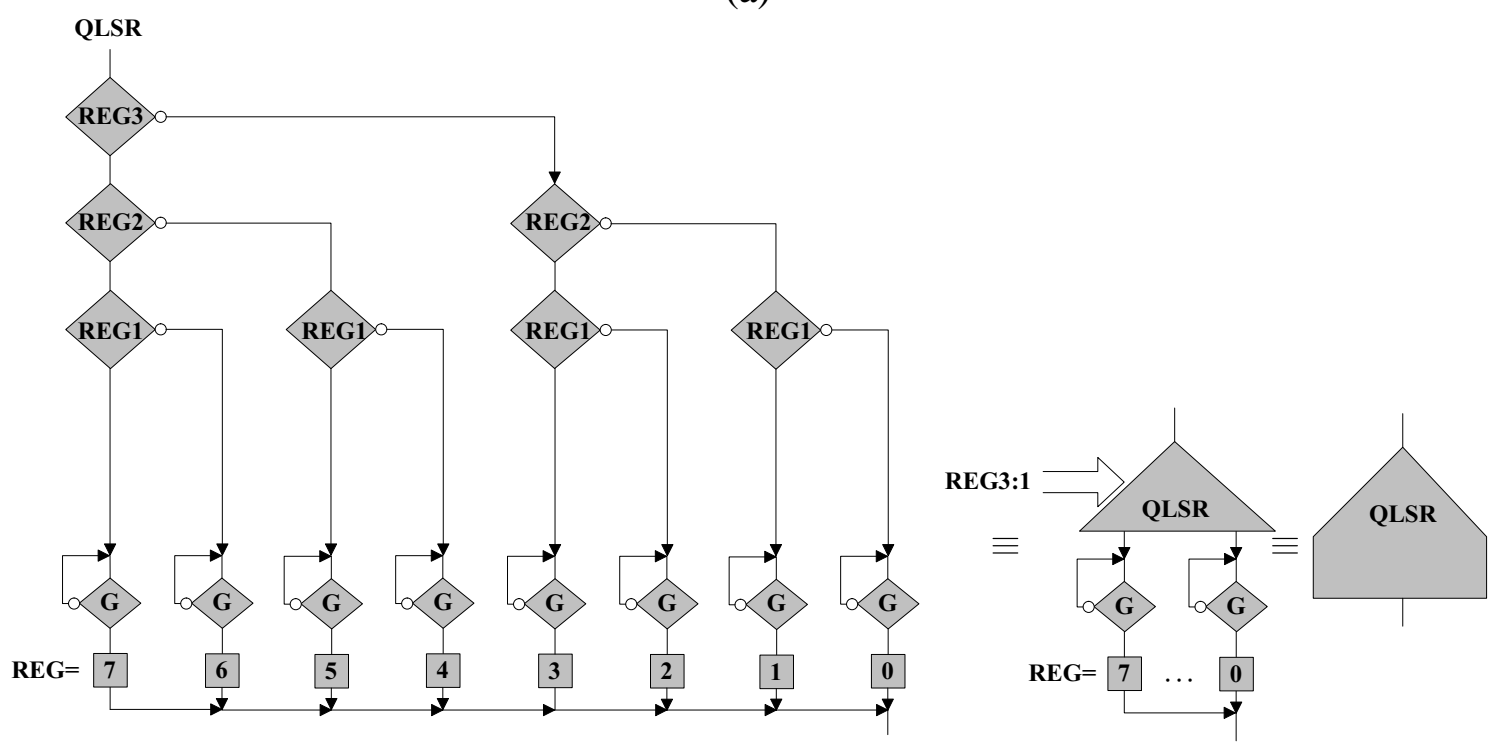

(b)

Figure 5.28: QL shift right computation. (a) Karnaugh map. (b) Binary decision diagram and flowcharts.

of the tree (inside the dashed square) implements the Karnaugh map of Figure 5.27a. After decoding the instruction, we need to implement the shift-left and the shift-right movements of the $Q R 0: 3$ cell.

To shift right the bits of the register ( $R E G)$ of the $Q R 0: 3$ cell, we need to know the value of this register. The Karnaugh map of Figure 5.31a decodes the value in the cell register and indicates the new value to be assigned to it, that is, the value after the shift right. Thus, if the current value is $R E G 3: 0=0110$, the next value will be $Q C 011$. Note that the leftmost bit is replaced by the bit 0 of the register $(R E G)$ of the $Q C$ cell. Finally, the Karnaugh map of Figure 5.31a is implemented by the binary decision diagram of Figure 5.31b.

To shift left the bits of the register (REG) of the $Q R O: 3$ cell, we need to know the 


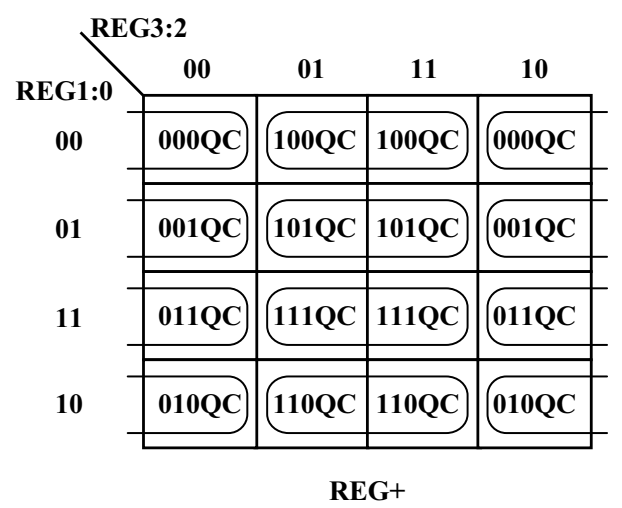

(a)

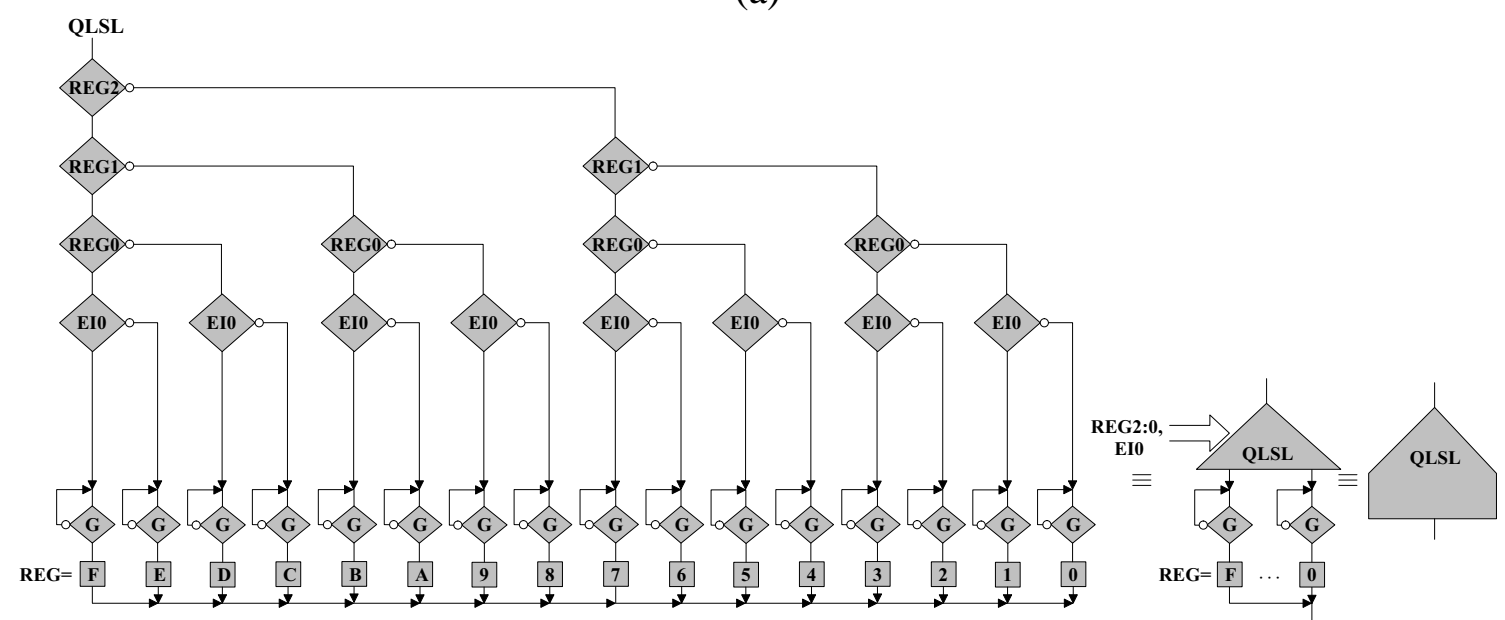

(b)

Figure 5.29: QL shift left computation. (a) Karnaugh map (b) Binary decision diagram and flowcharts.

value of this register. The Karnaugh map of Figure 5.32a decodes the value in the cell register and indicates the new value to be assigned to it, that is, the value after the shift left. Thus, if the current value is $R E G 3: 0=0110$, the next value will be 1100 . Note that we assume that the bit to the right of the $Q R O: 3$ cell has the value 0 . Finally, the Karnaugh map of Figure 5.32a is implemented by the binary decision diagram of Figure 5.32b.

\section{Computing the $Q C$ gene}

The Karnaugh map of Figure 5.33a decodes the PICOPASCAL instructions related to the implementation of the $Q C$ gene, that is, do $0 \rightarrow$, which indicates that the data tape has to move left, do $1 \leftarrow$, which indicates that the data tape has to move right, do 0 , which indicates that the register $(R E G)$ of the $Q C$ cell takes the value $0(Q C \leftarrow 0)$, and do 1 , which indicates that the register $(R E G)$ of the $Q C$ cell takes the value $1(Q C \leftarrow 1)$, otherwise it should not move, as shown in Figure 5.13.

The first test in the tree $(N I I)$ of Figure 5.33b corresponds to the test of the signal 

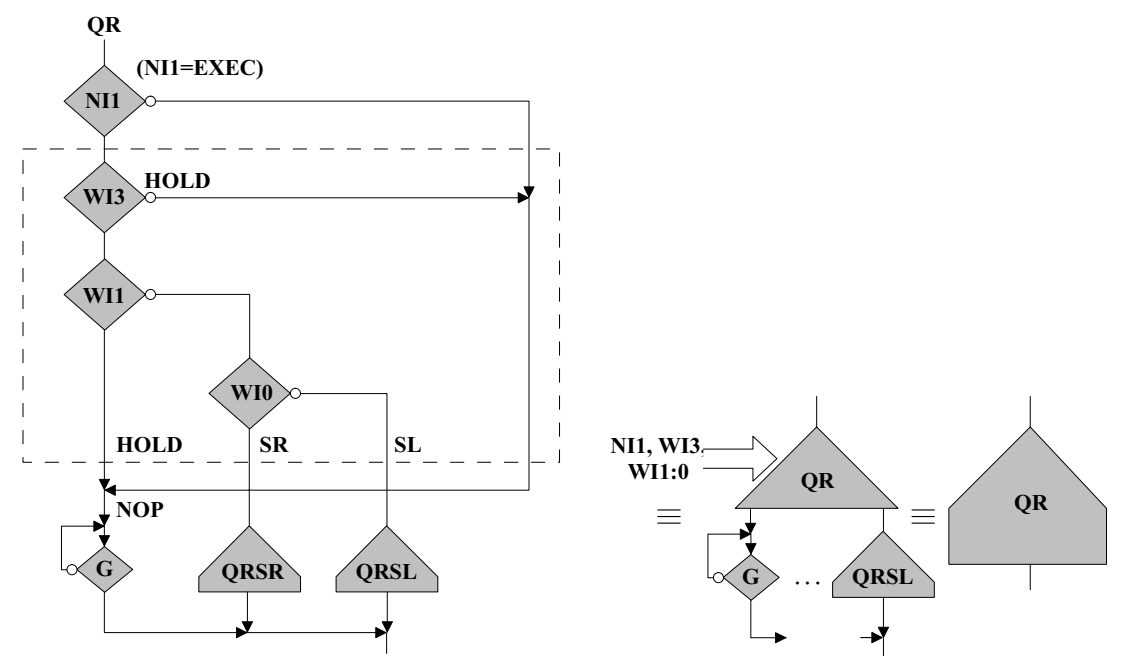

Figure 5.30: Binary decision diagram and flowcharts computing the QR gene.

\begin{tabular}{|c|c|c|c|c|}
\hline & 00 & 01 & 11 & 10 \\
\hline 00 & QCO00) & QC010 & QC110 & QC100 \\
\hline 01 & QC000 & QC010 & QC110 & QC100 \\
\hline 11 & QC001) & QC011) & QC111) & QC101 \\
\hline 10 & QC001 & QC011 & QC111 & QC101 \\
\hline
\end{tabular}

REG+

(a)

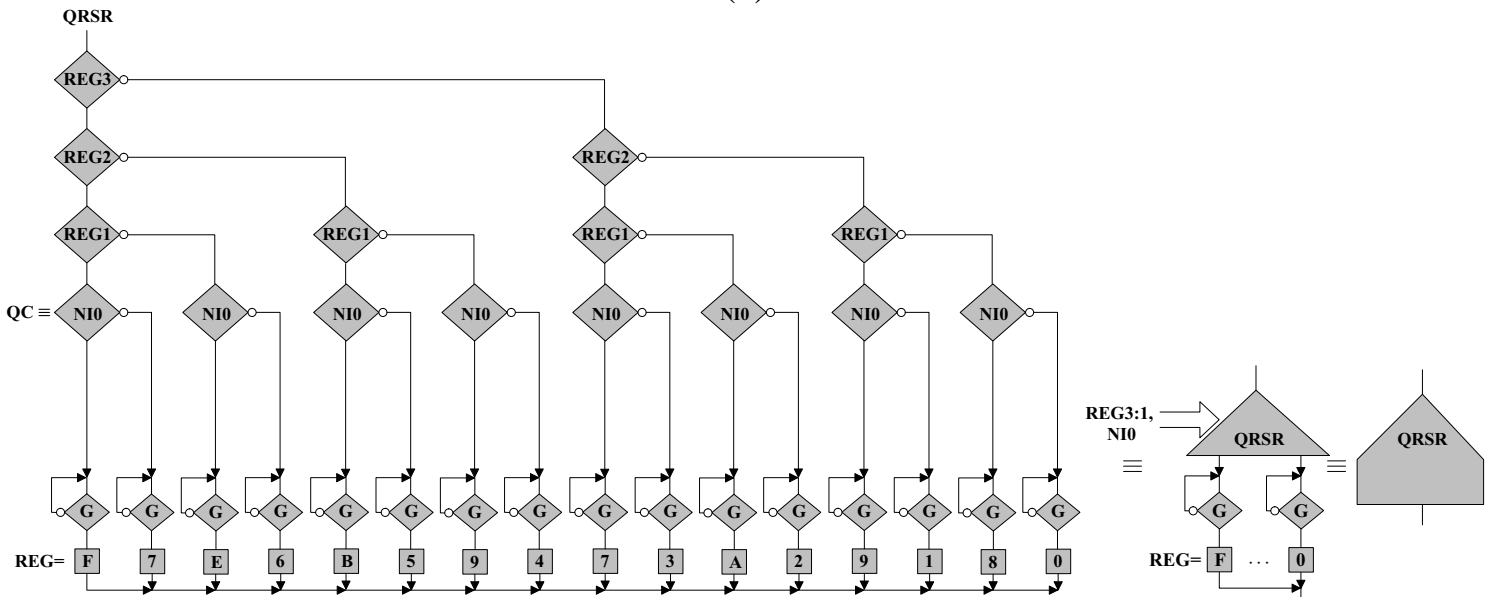

(b)

Figure 5.31: QR shift right computation. (a) Karnaugh map. (b) Binary decision diagram and flowcharts. 


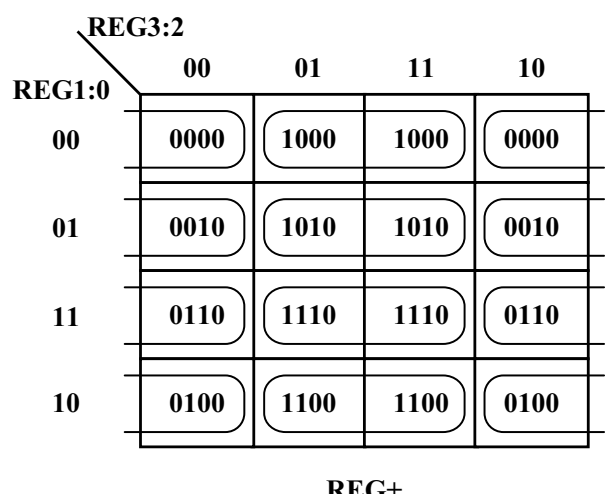

(a)
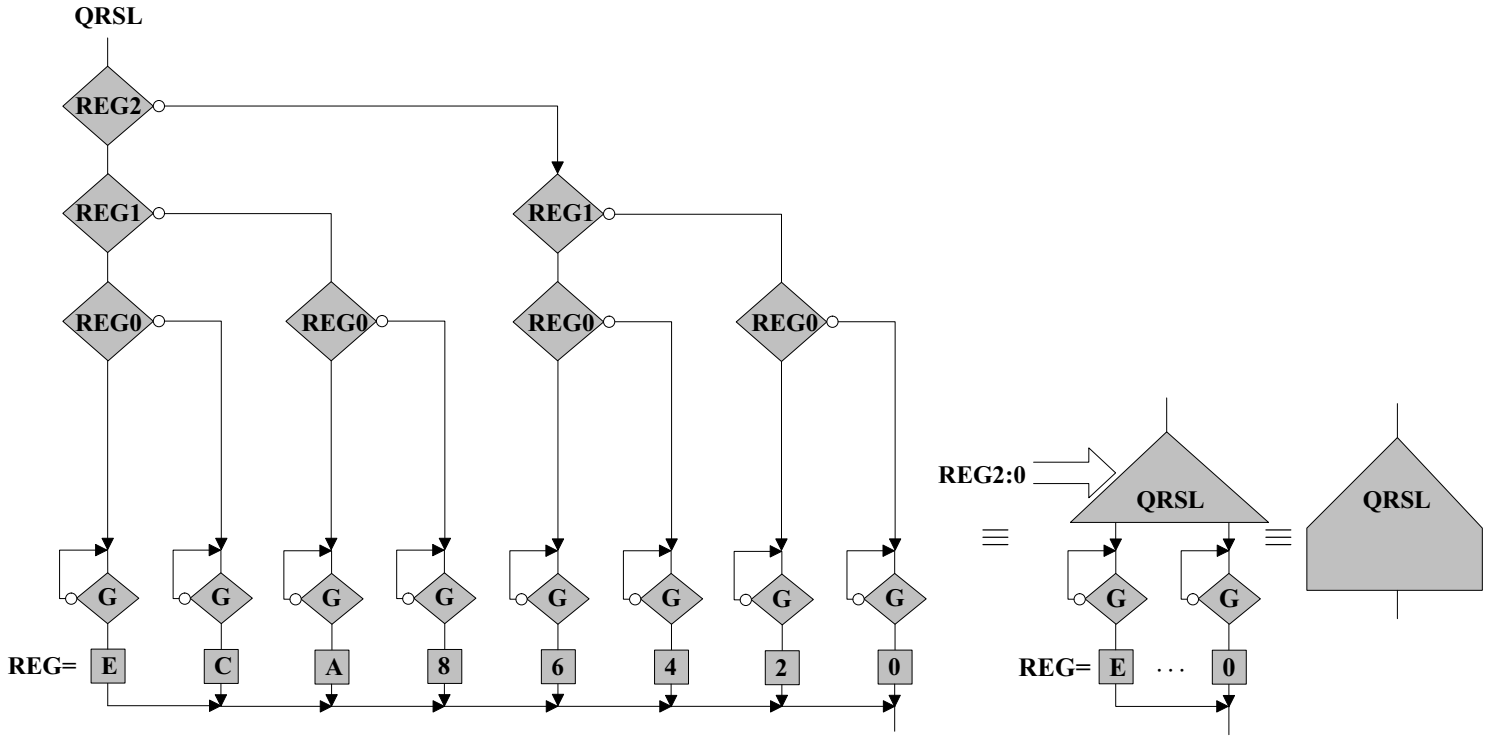

(b)

Figure 5.32: QR shift left computation. (a) Karnaugh map. (b) Binary decision diagram and flowcharts.

EXEC, which indicates if the assignment instruction will be executed or not. The sub-tree inside the dashed square implements the Karnaugh map of Figure 5.33a. Finally, after decoding the instruction, we implement the shift-left and the shift-right movements of the $Q C$ cell, as well as the $Q C \leftarrow 0$ and the $Q C \leftarrow 1$ assignments.

\subsubsection{Global configuration}

Our implementation does not have a global physical configuration because there is not a configuration realized in all the MICTREE cells of the array independently of the value of the coordinates $(X$ and/or $Y$ ). 


\begin{tabular}{|c|c|c|c|c|}
\hline WI1:0 & 00 & 01 & 11 & 10 \\
\hline 00 & $\Phi$ & HOLD & $\Phi$ & SL \\
\hline 01 & $\Phi$ & HOLD & $\Phi$ & SR \\
\hline 11 & $\Phi$ & $\Phi$ & $\Phi$ & $\mathrm{QC} \leftarrow 1$ \\
\hline 10 & HOLD & HOLD & $\Phi$ & $Q C \leftarrow 0$ \\
\hline
\end{tabular}

SL stands for SHIFT LEFT

QC

SR stands for SHIFT RIGHT

(a)

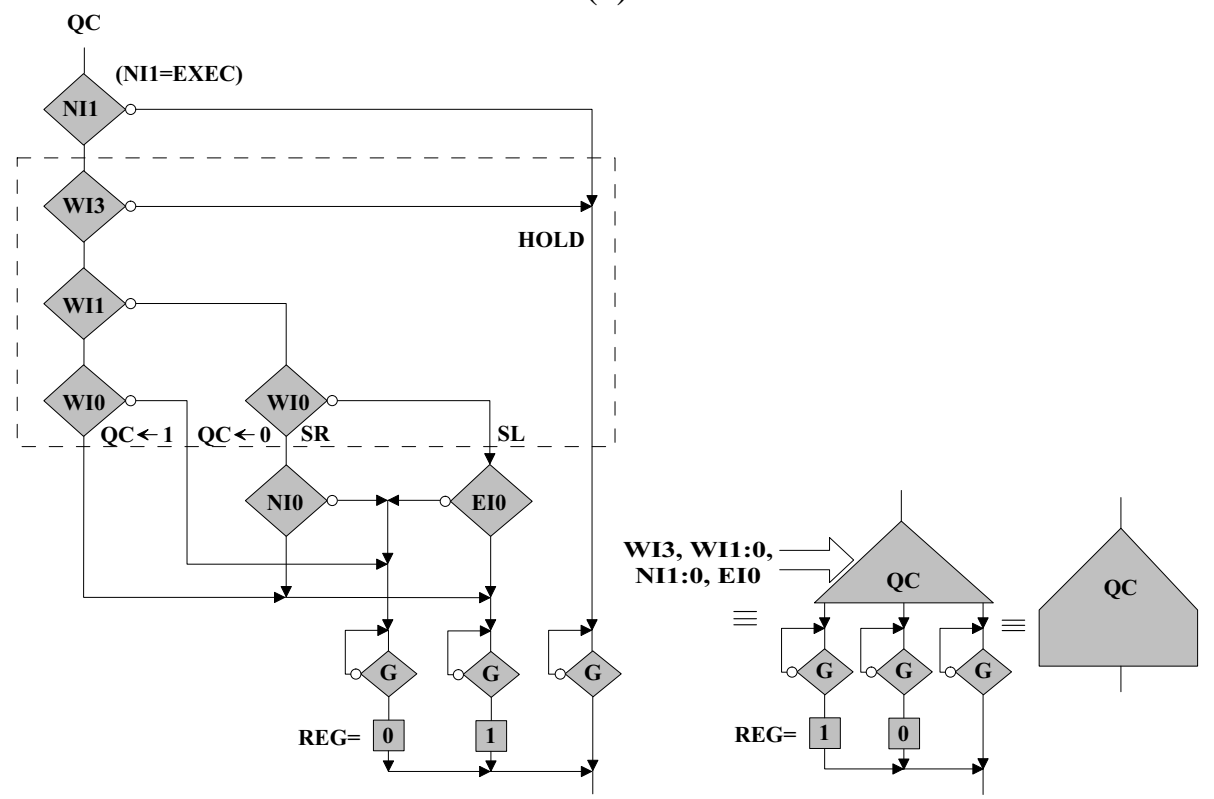

(b)

Figure 5.33: QC gene computation. (a) Karnaugh map decoding the instructions in column OPC in the operation table of Figure 5.13. (b) Binary decision diagram and flowcharts.

\subsubsection{Local configuration}

A physical configuration is local if it is realized by a sub-set of the MICTREE cells of the array. Such a configuration depends therefore on the value of the $X$ and/or $Y$ coordinates.

The architecture of our universal Turing machine (Figure 5.6) consists of three rows of cells, where different groups of cells implement specific parts of the organism (stack, register, data tape, and program tape), each part having, in general, two communications requirements: 
$\Rightarrow$ exchange of information with the others parts (stack, register, data tape, and program tape),

$\Rightarrow$ exchange of information among the cells within the same part.

To satisfy these requirements we need to identify clearly the different routing paths to be programmed for each cell or group of cells. That is, from what inputs the cell will receive the information coming from its neighbors, or through what outputs the cell will send the necessary information to its neighbors, or how the cell will forward the information coming through the cell but destined to another cell.

We will begin by analyzing the routing characteristics of the program tape cells. According to Figure 5.17:

$\Rightarrow$ the cells at coordinates $(W X=0 . .9, S Y=0)$ have to send the contents of their $R E G$ registers to the north neighbor through the output bus NO3:0. The corresponding assignment instructions may be written as follows:

$$
\begin{aligned}
\text { do } N O O & =R E G O \\
\text { do } N O 1 & =R E G 1 \\
\text { do } N O 2 & =R E G 2 \\
\text { do } N O 3 & =R E G 3
\end{aligned}
$$

$\Rightarrow$ the cells at coordinates ( $W X=1 . .9, S Y=1)$ have to send the contents of their $R E G$ registers to the west neighbor through the output bus WO3:0, have to convey the information coming from the south input bus SI3:0 to the east neighbor via the output bus EO3:0, and finally have to forward the information coming from the west input bus WI3:0 to the south neighbor through the output bus SO3:0. The corresponding assignment instructions may be written as follows:

$$
\begin{array}{lll}
\text { do } W O O=R E G O & \text { do } E O O=S I O & \text { do } S O O=W I O \\
\text { do } W O 1=R E G 1 & \text { do } E O 1=S I 1 & \text { do } S O 1=W I 1 \\
\text { do } W O 2=R E G 2 & \text { do } E O 2=S I 2 & \text { do } S O 2=W I 2 \\
\text { do } W O 3=R E G 3 & \text { do } E O 3=S I 3 & \text { do } S O 3=W I 3
\end{array}
$$

$\Rightarrow$ the cell at coordinates ( $W X=0, S Y=1$ ) has to send the contents of its $R E G$ register to the north neighbor through the output bus NO3:0 and to the south neighbor through the output bus SO3:0. The corresponding assignment instructions may be written as follows:

$$
\begin{array}{ll}
\text { do } N O O=R E G O & \text { do } S O O=R E G O \\
\text { do } N O 1=R E G 1 & \text { do } S O 1=R E G 1 \\
\text { do } N O 2=R E G 2 & \text { do } S O 2=R E G 2 \\
\text { do } N O 3=R E G 3 & \text { do } S O 3=R E G 3
\end{array}
$$


For the stack, register, and data tape parts, according to Figure 5.34, all the cells have the same routing needs, with the exception of $S T 3, S T 1, Q L$, and $Q R$ :

$\Rightarrow$ the $S T 2, Q, S$, and $Q C$ cells have to send the contents of REGO (bit 0 of the REG register) to the north and west neighbors through the $N O O$ and $W O O$ outputs respectively, and to convey the information coming from the west input bus WI3:0 to the east neighbor via the output bus EO3:0. The corresponding assignment instructions may be written as follows (Figure 5.34a):

$$
\begin{array}{ll}
\text { do } N O O=R E G O & \text { do } E O O=W I O \\
\text { do } W O O=R E G O & \text { do } E O 1=W I 1 \\
& \text { do } E O 2=W I 2 \\
\text { do } E O 3=W I 3
\end{array}
$$

$\Rightarrow$ the $S T 1$ cell has to send the contents of REGO to the north and west neighbors through the $N O O$ and $W O O$ outputs respectively, has to convey the information coming from the EIO input to the west neighbor via the WOI output, and has to forward the information coming from the west input bus WI3:0 to the east neighbor via the output bus EO3:0. The corresponding assignment instructions may be written as follows (Figure 5.34b):

$$
\begin{array}{ll}
\text { do } N O O=R E G O & \text { do } E O O=W I O \\
\text { do } W O O=R E G O & \text { do } E O 1=W I 1 \\
\text { do } W O 1=E I O & \text { do } E O 2=W I 2 \\
& \text { do } E O 3=W I 3
\end{array}
$$

$\Rightarrow$ the $S T 3$ cell has to send the contents of REGO to the north neighbor through the NOO outputs, and has to convey the information coming from the south input bus SI3:0 to the east neighbor via the output bus EO3:0. The corresponding assignment instructions may be written as follows (Figure 5.34c):

$$
\text { do } N O O=R E G O \quad \begin{aligned}
& \text { do } E O O=S I O \\
& \text { do } E O 1=S I 1 \\
& \text { do } E O 2=S I 2 \\
& \text { do } E O 3=S I 3
\end{aligned}
$$

$\Rightarrow$ the $Q L$ cell has to send the contents of REGO to the north neighbor through the NOO output, has to convey the information coming from the $E I O$ to the west neighbor via the output $W O O$, and has to forward the information coming from the west input bus WI3:0 to the east neighbor via the output bus EO3:0. The corresponding assignment instructions may be written as follows (Figure 5.34d):

$$
\begin{array}{ll}
\text { do } N O O=R E G O & \text { do } E O O=W I O \\
\text { do } W O O=E I O & \text { do } E O 1=W I 1 \\
& \text { do } E O 2=W I 2 \\
\text { do } E O 3=W I 3
\end{array}
$$




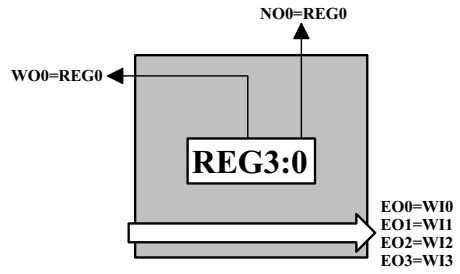

(a)

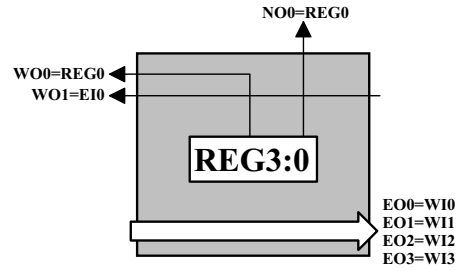

(b)

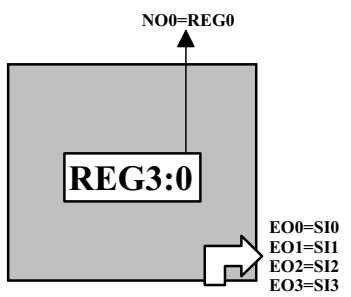

(c)

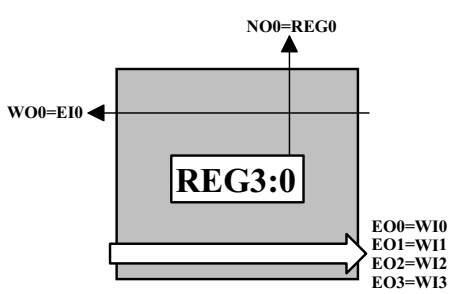

(d)

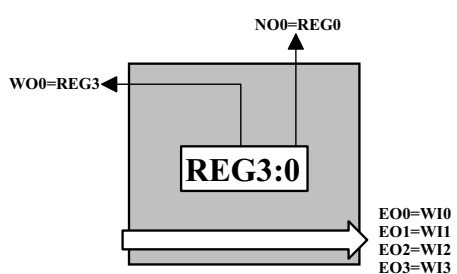

(e)

Figure 5.34: Local configuration of stack, register, and data tape cells $(Y=0)$. (a) Configuration of cells ST2, Q,S, and QC. (b) Configuration of cell ST1. (c) Configuration of cell ST3. (d) Configuration of cell QL. (e) Configuration of cell $Q R$.

$\Rightarrow$ the $Q R$ cell has to send the contents of $R E G O$ and $R E G 3$ to the north and west neighbors through the $N O O$ and $W O O$ outputs respectively, and to convey the information coming from the west input bus WI3:0 to the east neighbor via the output bus EO3:0. The corresponding assignment instructions may be written as follows(Figure 5.34e):

$$
\begin{array}{ll}
\text { do } N O O=R E G O & \text { do } E O O=W I O \\
\text { do } E O 1=W I 1 \\
\text { do } W O O=R E G 3 & \text { do } E O 2=W I 2 \\
\text { do } E O 3=W I 3
\end{array}
$$

To compute the local configuration, i.e., to set each cell of the cellular space on a particular configuration, we need a methodology that is roughly the same as the one used to compute the coordinates. That is, we need to know what the horizontal and vertical coordinates of each cell are, in order to configure it correctly. Therefore, we can reuse the XYInitialConfig sub-program to differentiate the cells we need to configure. Modifying the XYInitialConfig sub-program of Figure 5.16, we obtain the new sub-program XYInitialLocalConfig of Figure 5.35, which implements, after some optimizations, configurations 5.1 to 5.8 . 


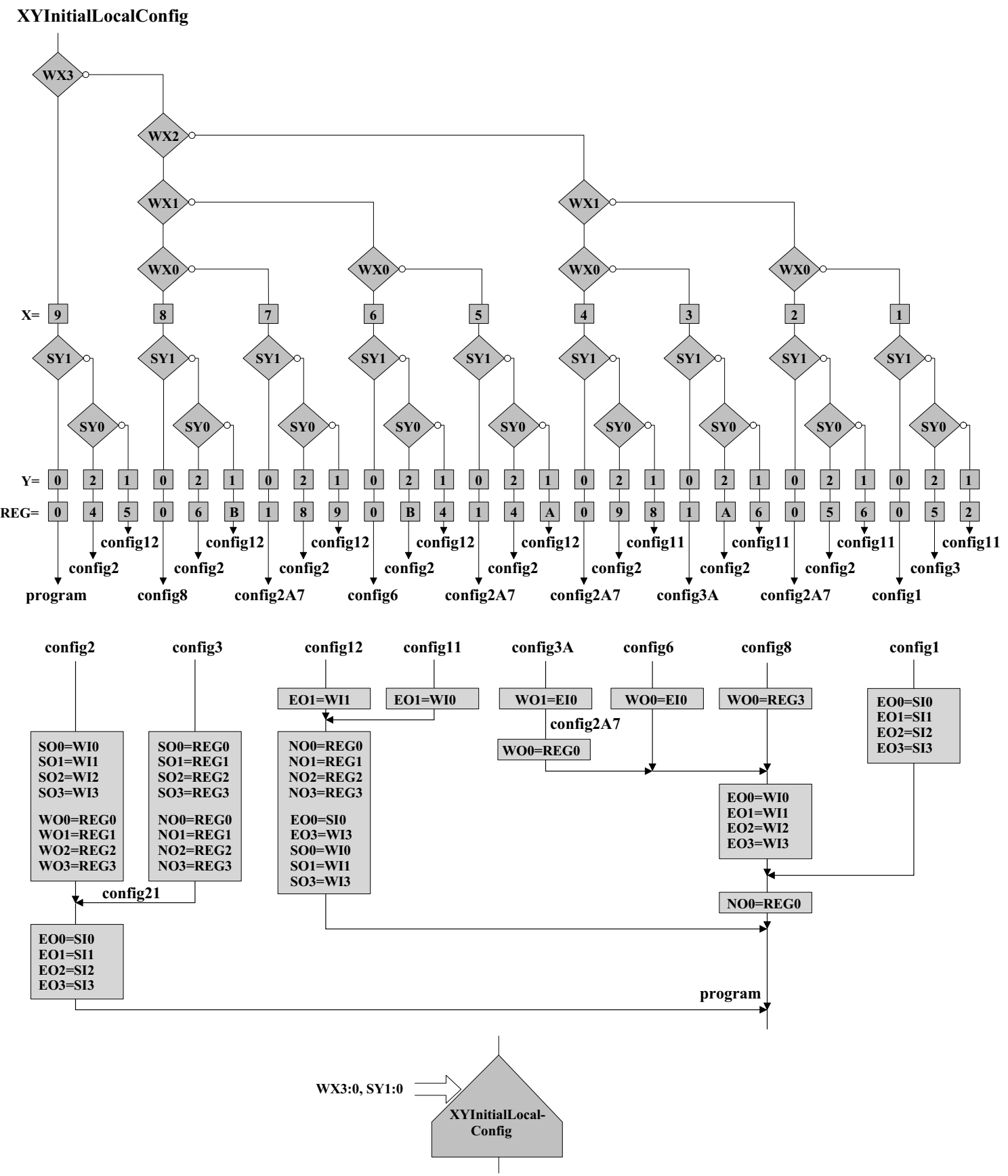

Figure 5.35: XYInitialLocalConfig binary decision diagram and flowchart resulting from reusing the XYInitialConfig sub-program (Figure 5.16) to compute the local configuration.

\subsubsection{Final microprogram}

The complete genome is represented by the final flowchart UTMgenome of Figure 5.36. It starts with initial conditions assuring that: 
$\Rightarrow$ all the state registers of the array are set to $0(R E G=0)$;

$\Rightarrow$ the coordinates $X$ and $Y$ are set to $0(X=0, Y=0)$.

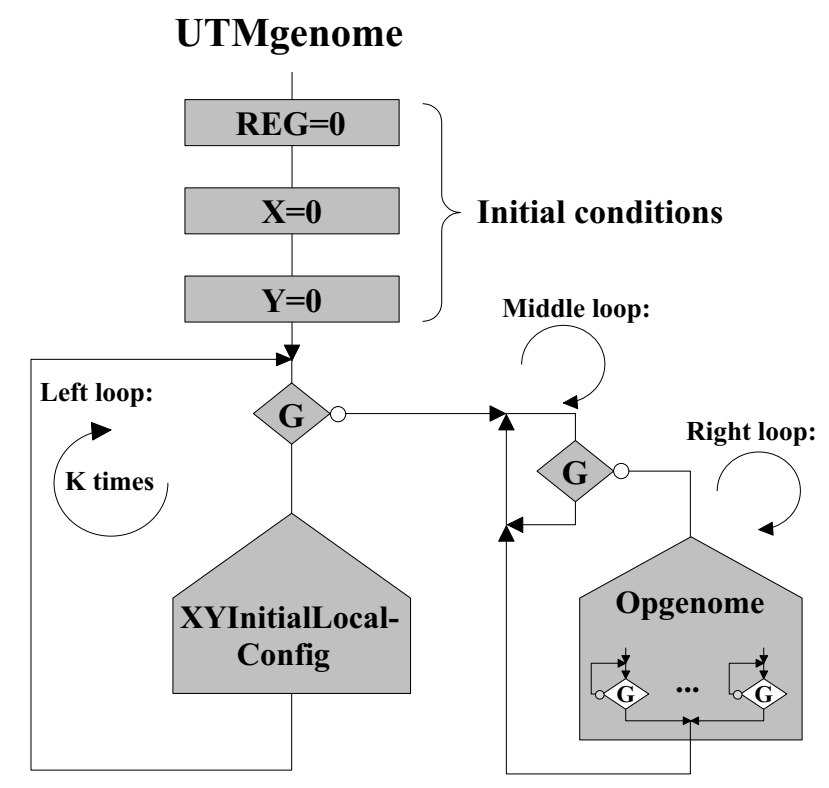

Figure 5.36: Complete genome microprogram (UTMgenome) flowchart.

The microprogram then executes one of three loops, controlled by the variable $G$ (the global clock):

1. For the first period of $G$, while $G=1$, at least $K$ executions of the left-hand loop are necessary to ensure that the rightmost and the uppermost cells of the organism correctly compute their coordinates, their initial conditions, and their local configurations (as defined by the sub-program XYInitialLocalConfig). $K$ is the largest of $X \max$ and Ymax (Xmax and Ymax are, respectively, the maximum number of rows and columns of the artificial organism). In our example, $K=10$ (Figure 5.7).

2. At the falling edge of $G(G=1 \rightarrow 0)$, the right loop is selected and the operational part of the genome is executed. To assure the synchronization of all the cells, tests are performed throughout the half-period when $G=0$, but no assignment is made until the rising edge of $G(G=0 \rightarrow 1)$, when all the registers $R E G$ are updated simultaneously.

3. At the rising edge of $G(G=0 \rightarrow 1)$, after the update of the registers, the middle loop is executed. The cellular automaton stays in this loop until the next falling edge of $G(G=1 \rightarrow 0)$ arrives, selecting the right-hand loop and starting a new cycle. 


\subsection{Experiment and discussion}

The UTM was completely implemented and the binary counter fully tested. Table 5.1 shows the contents of the REG register of cells $Q$ and $Q L 3: 0, Q C, Q R 0: 3$ (the data tape) at the end of each program execution cycle. The values obtained correspond exactly to the results presented by Minsky in [64], assuming that the tape register is limited to 9 bits with fixed boundary conditions ( $R=L=0$ in Figure 5.10). The measured sequence ends in a final 0000 state (in hexadecimal), which constitutes a fixed point for the system.

The complete genome microprogram describing our artificial organism is composed of 377 16-bit-wide instructions, implying a configuration bit string of 6032 bits. The listing of the complete genome microprogram can be found in Appendix B.

We tested the self-repair capabilities of our implementation (Figure 5.6), made possible by the spare column at the right edge of our artificial organism. Using this spare column, our organism is able to tolerate at least one fault in any cell of the array, and up to three faulty cells in the same column.

From the flowchart describing the genome microprogram (Figure 5.36), we can note that our artificial organism should execute at least $K$ times the left-hand loop while $G=1$ during initialization. After initialization, the organism will always execute the middle or right loop depending on the global clock $G$ (the left-loop will never be executed again). Thus, when a fault is detected and the self-repair process is over, it will be necessary to reinitialize (manually) the organism to make it operational once again.

To solve this problem we could imagine a different implementation in which we use another external global signal (INIT, for example) to indicate to our artificial organism that, after the self-repair process is over, it has to re-execute the left loop. Figure 5.37 illustrates this possible solution.

Even better, we could imagine a solution where the MICTREE state is not lost when a fault is detected. In this case, because no information was lost, we can maintain the state and the functionality of the system without any re-initialization. A very interesting research on this topic was realized by Madon in [45].

Unfortunately, these two improvements (a global signal INIT and saving the cell state) are not realizable with the actual MICTREE architecture, the main problem being the poor routing resources of the MICTREE cell.

The self-replication of our UTM was tested with one copy of the original organism, as shown Figure 5.7. For this test, the cellular array contained $6 \times 10=60$ MICTREE cells (Figure 5.38).

The largest possible artificial organism implementation with cellular differentiation can reach a size of 16x16 MICTREE cells, a limit imposed by the architecture of the cell. This limitation disappears if we realize a molecular decomposition of the MICTREE cell, allowing us to tailor the cell to meet the requirements of any application $[52,55]$. 


\section{UTMgenome}

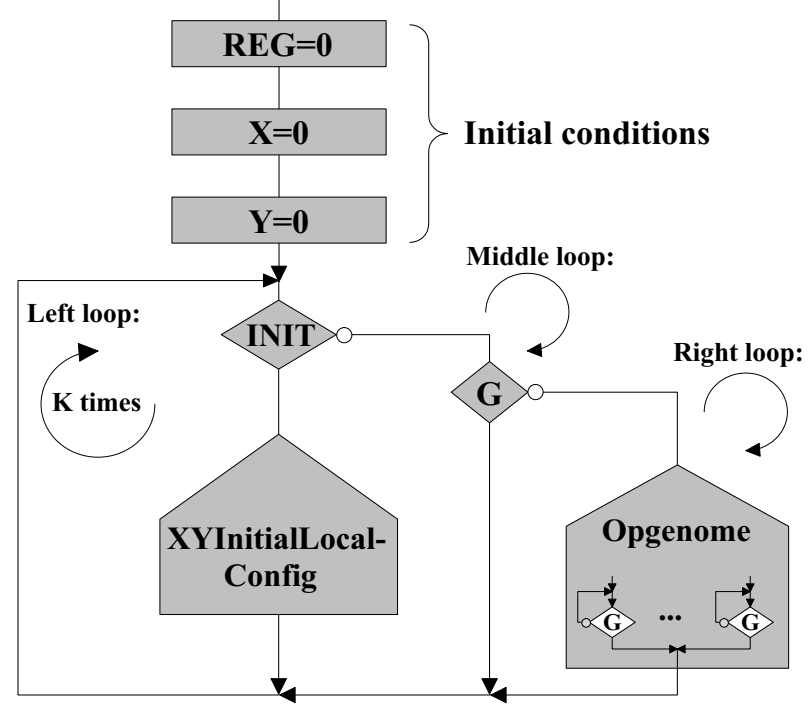

Figure 5.37: Possible genome microprogram (UTMgenome) flowchart with a second external global signal INIT.

Table 5.1: Experimental results

\begin{tabular}{|c|c|c|c|c|c|c|}
\hline state & \multicolumn{3}{|c|}{ data tape (hexa) } & \multicolumn{3}{|c|}{ data tape (binary) } \\
\hline$Q$ & QL3:0 & $Q C$ & $Q R 0: 3$ & QL3:0 & $Q C$ & $Q R 0: 3$ \\
\hline 0 & 0 & 1 & 0 & 0000 & 1 & 0000 \\
\hline 1 & 0 & 0 & 8 & 0000 & 0 & 1000 \\
\hline 0 & 1 & 1 & 0 & $\begin{array}{llll}0 & 0 & 0\end{array}$ & 1 & 0000 \\
\hline 1 & 0 & 1 & 8 & 0000 & 1 & 1000 \\
\hline 1 & 0 & 0 & 4 & 0000 & 0 & 0100 \\
\hline 0 & 1 & 0 & 8 & 0001 & 0 & 1000 \\
\hline 0 & 2 & 1 & 0 & 0010 & 1 & 0000 \\
\hline 1 & 1 & 0 & 8 & 00001 & 0 & 1000 \\
\hline 0 & 3 & 1 & 0 & 0011 & 1 & 0000 \\
\hline 1 & 1 & 1 & 8 & 0001 & 1 & 1000 \\
\hline 1 & 0 & 1 & 4 & 0000 & 1 & 0100 \\
\hline 1 & 0 & 0 & 2 & 0000 & 0 & 0010 \\
\hline 0 & 1 & 0 & 4 & 0001 & 0 & 0100 \\
\hline 0 & 2 & 0 & 8 & 0010 & 0 & 1000 \\
\hline 0 & 4 & 1 & 0 & 0100 & 1 & 0000 \\
\hline 1 & 2 & 0 & 8 & 0010 & 0 & 1000 \\
\hline 0 & 5 & 1 & 0 & 0101 & 1 & 0000 \\
\hline 1 & 2 & 1 & 8 & 0010 & 1 & 1000 \\
\hline 1 & 1 & 0 & 4 & 0001 & 0 & 0100 \\
\hline
\end{tabular}


Table 5.1: Experimental results

\begin{tabular}{|c|c|c|c|c|c|c|}
\hline state & \multicolumn{3}{|c|}{ data tape (hexa) } & \multicolumn{3}{|c|}{ data tape (binary) } \\
\hline$Q$ & QL3:0 & $Q C$ & $Q R 0: 3$ & QL3:0 & $Q C$ & QR0:3 \\
\hline 0 & 3 & 0 & 8 & 0011 & 0 & 1000 \\
\hline 0 & 6 & 1 & 0 & 0110 & 1 & 0000 \\
\hline 1 & 3 & 0 & 8 & 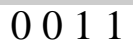 & 0 & 1000 \\
\hline 0 & 7 & 1 & 0 & 01111 & 1 & 0000 \\
\hline 1 & 3 & 1 & 8 & 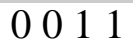 & 1 & 1000 \\
\hline 1 & 1 & 1 & 4 & 0001 & 1 & 0100 \\
\hline 1 & 0 & 1 & 2 & 0000 & 1 & 0010 \\
\hline 1 & 0 & 0 & 1 & 0000 & 0 & 0001 \\
\hline 0 & 1 & 0 & 2 & 00001 & 0 & 0010 \\
\hline 0 & 2 & 0 & 4 & 0010 & 0 & 0100 \\
\hline 0 & 4 & 0 & 8 & 0100 & 0 & 1000 \\
\hline 0 & 8 & 1 & 0 & 1000 & 1 & 00000 \\
\hline 1 & 4 & 0 & 8 & 0100 & 0 & 1000 \\
\hline 0 & 9 & 1 & 0 & 1001 & 1 & 00000 \\
\hline 1 & 4 & 1 & 8 & 0100 & 1 & 1000 \\
\hline 1 & 2 & 0 & 4 & 0010 & 0 & 0100 \\
\hline 0 & 5 & 0 & 8 & 0101 & 0 & 1000 \\
\hline 0 & A & 1 & 0 & 1010 & 1 & 0000 \\
\hline 1 & 5 & 0 & 8 & 0101 & 0 & 1000 \\
\hline 0 & B & 1 & 0 & 1011 & 1 & 0000 \\
\hline 1 & 5 & 1 & 8 & 0101 & 1 & 1000 \\
\hline 1 & 2 & 1 & 4 & 0010 & 1 & 0100 \\
\hline 1 & 1 & 0 & 2 & 0001 & 0 & 0010 \\
\hline 0 & 3 & 0 & 4 & 0011 & 0 & 0100 \\
\hline 0 & 6 & 0 & 8 & 0110 & 0 & 1000 \\
\hline 0 & $\mathrm{C}$ & 1 & 0 & 1100 & 1 & 0000 \\
\hline 1 & 6 & 0 & 8 & 0110 & 0 & 1000 \\
\hline 0 & D & 1 & 0 & 1101 & 1 & 0000 \\
\hline 1 & 6 & 1 & 8 & 0110 & 1 & 1000 \\
\hline 1 & 3 & 0 & 4 & $\begin{array}{llll}0 & 0 & 11\end{array}$ & 0 & 0100 \\
\hline 0 & 7 & 0 & 8 & 01011 & 0 & 1000 \\
\hline 0 & $\mathrm{E}$ & 1 & 0 & 1110 & 1 & 0000 \\
\hline 1 & 7 & 0 & 8 & $\begin{array}{llll}0 & 1 & 11\end{array}$ & 0 & 1000 \\
\hline 0 & $\mathrm{~F}$ & 1 & 0 & 1111 & 1 & 0000 \\
\hline 1 & 7 & 1 & 8 & $\begin{array}{llll}0 & 1 & 11\end{array}$ & 1 & 1000 \\
\hline 1 & 3 & 1 & 4 & 0011 & 1 & 0100 \\
\hline 1 & 1 & 1 & 2 & 0001 & 1 & 0010 \\
\hline 1 & 0 & 1 & 1 & 0000 & 1 & 0001 \\
\hline 1 & 0 & 0 & 0 & 0000 & 0 & 0000 \\
\hline 0 & 1 & 0 & 0 & 0001 & 0 & 0000 \\
\hline
\end{tabular}

continued on next page ... 
Table 5.1: Experimental results

\begin{tabular}{|c|c|c|c|c|c|c|}
\hline state & \multicolumn{3}{|c|}{ data tape (hexa) } & \multicolumn{3}{|c|}{ data tape (binary) } \\
\hline$Q$ & QL3:0 & $Q C$ & $Q R 0: 3$ & QL3:0 & $Q C$ & QRO:3 \\
\hline 0 & 2 & 0 & 0 & 0010 & 0 & $\begin{array}{llll}0 & 0 & 0 & 0\end{array}$ \\
\hline 0 & 4 & 0 & 0 & 0100 & 0 & 0000 \\
\hline 0 & 8 & 0 & 0 & 1000 & 0 & 0000 \\
\hline 0 & 0 & 0 & 0 & 0000 & 0 & 0000 \\
\hline 0 & 0 & 0 & 0 & 0000 & 0 & 0000 \\
\hline
\end{tabular}

\subsection{Conclusion}

In this chapter we showed that it is possible to embed a universal Turing machine into a multicellular array based on MICTREE artificial cells, thus obtaining a self-repairing and self-replicating universal Turing machine.

The mapping of the universal Turing machine onto our multicellular array was made possible thanks to the introduction of a modified version of the W-machine [112], i.e., an interpreter of the PICOPASCAL language. We showed that an ideal architecture (i.e., an architecture with a semi-infinite data tape) was able to deal with applications of any complexity. We also presented an actual implementation in which we relaxed somewhat the requirements of the ideal architecture in order to use a smaller number of our MICTREE artificial cells. We slightly simplified our implementation by presenting the example of the binary counter in which the data are binary-coded and where the direction of the head's motion coincides with the internal state (in general, functions $Q+$ and $D+$ are independent). A picture of the final implementation is shown in Figure 5.38.

The property of universal construction raises issues of a different nature, since it requires (according to von Neumann) that a MICTREE cell be able to implement organisms of any dimension. This challenge, which lies outside of the scope of this thesis, can be met by decomposing a cell into molecules and tailoring the structure of cells to the requirements of a given application $[52,55]$. 


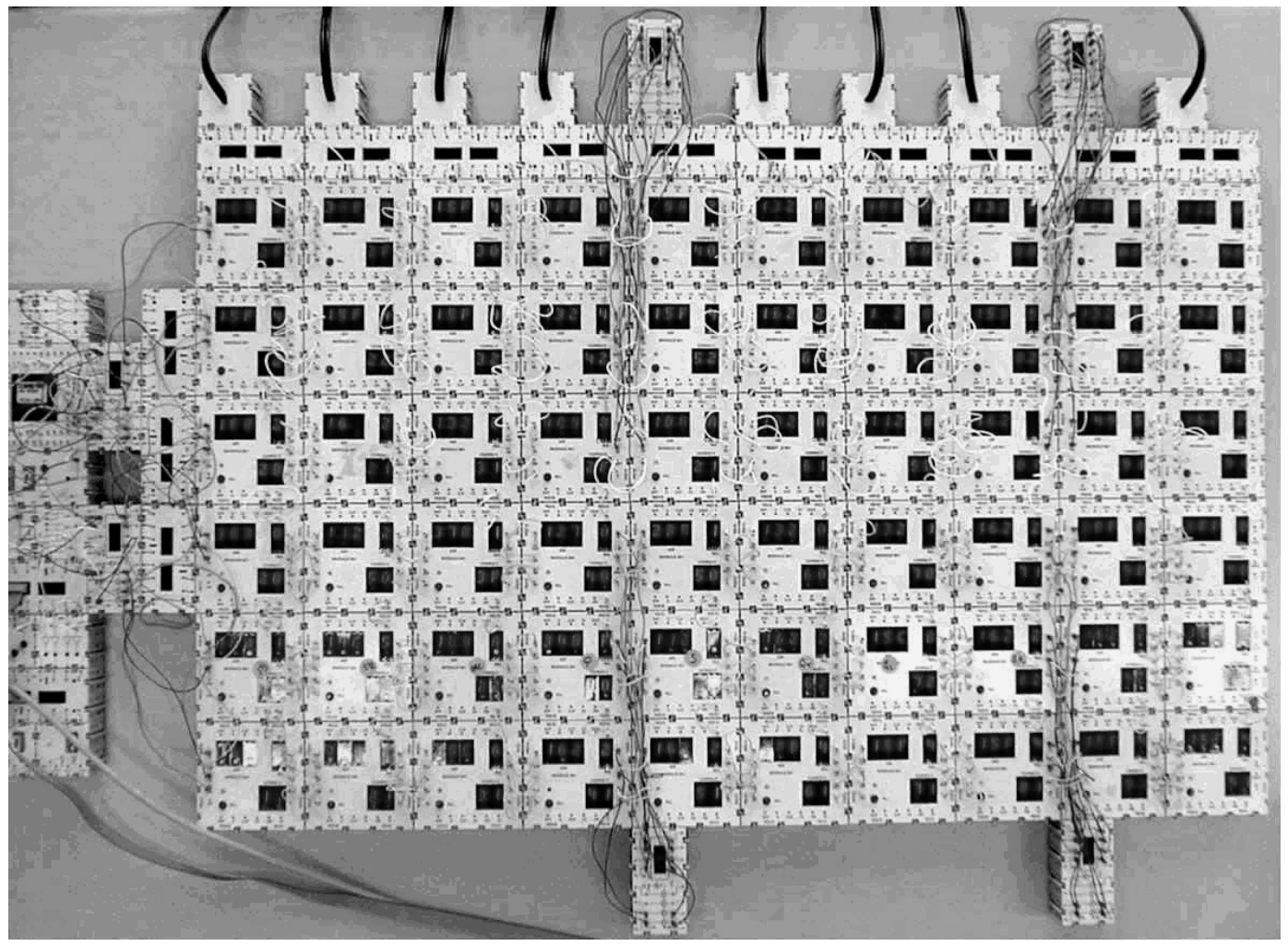

Figure 5.38: Final universal Turing machine implementation. This implementation contains six rows and ten columns of MICTREE cells, allowing us to verify self-replication (one copy) and self-repair (one spare column). 


\section{Chapter 6}

\section{Software Tools}

As we could see from the examples in Chapter 3 (the random number generator and the 2by-n hybrid cellular automaton), and in Chapters 4 (the specialized Turing machine) and 5 (the universal Turing machine), while the MICTREE cells can be used to create complex artificial organisms, the design of the software programs defining their behavior is difficult and has to be done by hand.

In this chapter we present a description of the different software tools that have been developed to deal with the programming complexity of implementing artificial organisms in the Embryonics architecture. Thus, in Sections 6.1, 6.2, and 6.3 we describe the main software tools that have been developed (MIC Editor, MIC Compiler, Visual BDD, MIC Sim, and the serial loader). Section 6.3 will present in particular detail the MICTREE network simulator software, code-named MIC Sim. In Section 6.4 we present the Visual MICTREE design environment, a single graphical design environment in which all the above-mentioned software tools have being integrated. Finally, in Section 6.5 we present the main features of Visual MICTREE by re-designing the 2-by-n hybrid cellular automaton example.

\subsection{MIC Editor and MIC Compiler}

MIC Editor is a general-purpose easy-to-use text editor for the MIC language (the language used to program the MICTREE artificial cell). It also offers many useful features for programmers, such as syntax highlighting, dedicated toolbars, line numbering, search and replace, keyboard shortcuts, and more.

The MIC Compiler, which integrates the MIC Editor program, is a compiler for the MIC language. The output of the compiler is a genome microprogram to be executed by the MICTREE binary decision machine. After compiling the design, the programmer can proceed to simulate the design with MIC Sim (Section 6.3) or to download the genome microprogram into an actual MICTREE network. 


\subsection{Visual BDD}

Visual BDD [65] is a graphical environment used to create flowcharts describing MICTREE genome microprograms. Visual BDD is composed mainly of a binary decision diagram (BDD) editor, a flowchart components editor, and a MIC code generator (Figure 6.1).

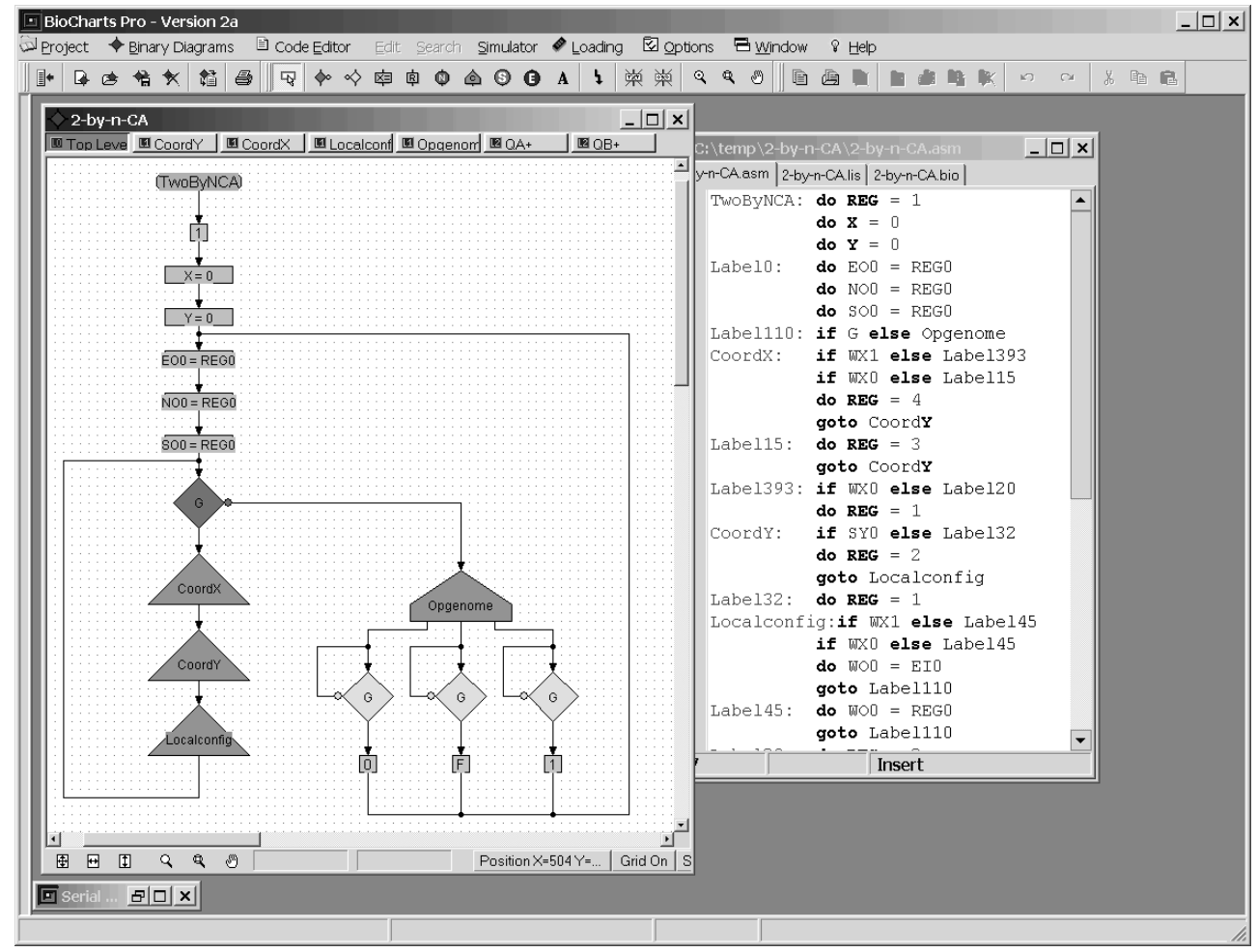

Figure 6.1: The left window shows a flowchart describing an artificial organism. The right window shows the corresponding code generated by the MIC code generator.

This program presents a very easy-to-use and powerful graphical design environment, allowing the user to deal with complex BDD designs. One of the most interesting features of Visual BDD is the possibility of having hierarchical design views, i.e., to describe the genome microprogram at different levels. One example is presented in Figure 6.2, where we see the window describing the high-level view (at the center) and other windows around it describing lower-level views. This functionality allows an easier and faster development of MICTREE genome microprograms, which can be sent through the computer's serial port, using a serial loader, to configure an actual MICTREE network, or can be used directly by the MIC Sim simulator described in the next section. 


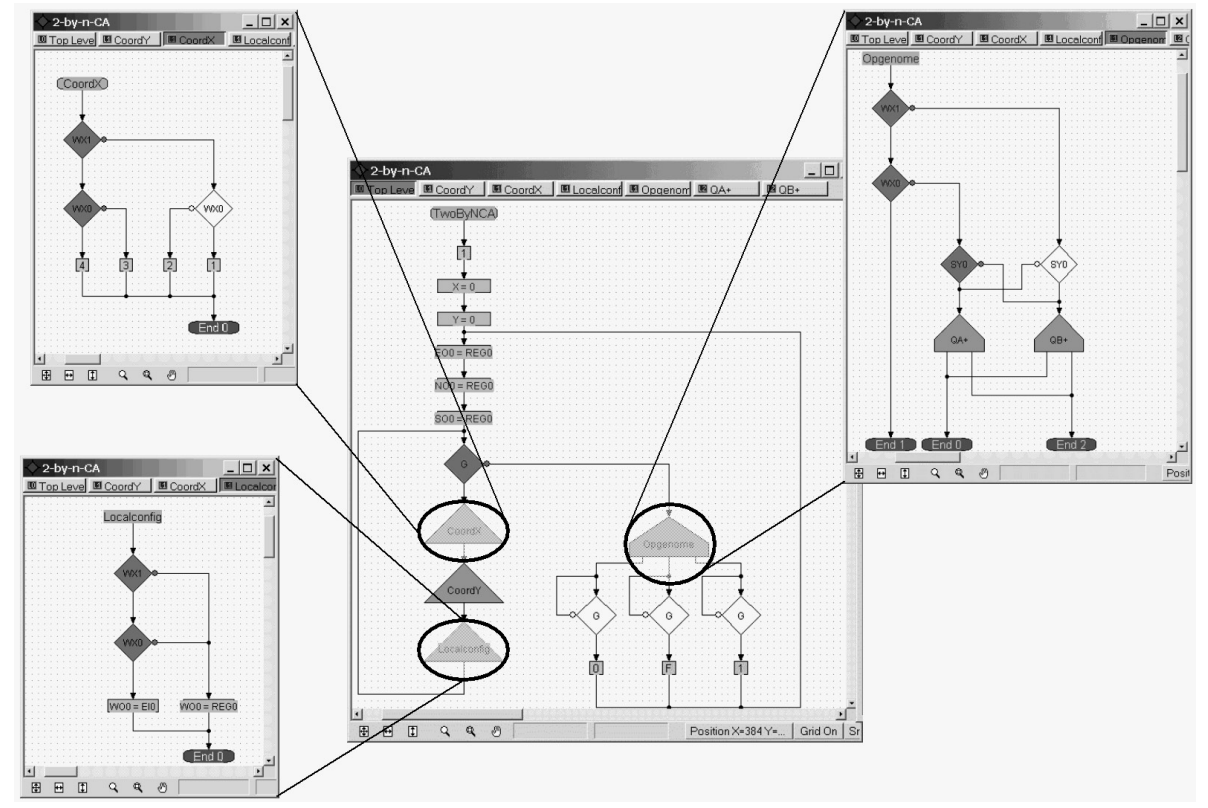

Figure 6.2: Hierarchical design views. The windows around the center window (high-level view) are lower-level views describing the contents of some component of the high-level view.

\subsection{MIC Sim}

MIC Sim is a MICTREE network simulator [27, 116, 117] that allows us to study the behavior of our artificial organism (the MICTREE network) before the actual implementation. The purpose of the simulator is to relieve the MICTREE user of all the problems involved in the use and management of the real MICTREE network. The user will be able to devote his attention to the development of the application rather than to the physical implementation.

At the moment there exist two versions of MIC Sim, the first implemented with Borland $\mathrm{C}++$ Builder, and the second in Java. The goal of the first version is to offer a powerful simulation tool integrated into a graphical environment running on a PC, whereas the goal of the Java implementation is to allow a stand-alone simulation of an artificial organism on an Internet browser. The Java implementation will not be described here.

With MIC Sim, the user can easily describe the MICTREE network topology to be simulated and use the genome microprogram generated by the MIC Compiler to configure the MICTREE network and start the simulation.

In this section we describe in detail the implementation of the MICTREE network simulator. We will emphasize the object model behind the simulator rather that the graphic interface features. 
The development of the simulator occurred in several phases. First, we sought the best way to implement the simulation engine, which is the heart of our tool, then we modelled a network of MICTREE cells, and finally we developed the graphic interface.

\subsubsection{Simulation engine}

The execution of a genome by a cellular network is controlled by a global clock. Therefore, one can consider that time is not continuous but that it advances in discrete steps. This is why we opted for simulation by discrete events [20]. Within this approach, there are two possible types of simulators: event-oriented simulators and process-oriented simulators.

In the first case, the event is the central part of the simulator: for each event that occurs, the simulator reacts and carries out an action, in theory, by activating a procedure. This approach is the traditional approach to simulation by discrete events.

The second type of simulator, the process-oriented simulator, is better suited for the simulation of a cellular network. The basic principle is the following: a process is created for each cell and at each rising edge of the clock all the processes are activated, then deactivated as soon as they are completed their task. They thus operate in a quasi-parallel manner.

The second kind of simulator is the one that better models the behavior of our MICTREE network, and we therefore opted for this type of simulator. The details of the simulator's implementation will not be given here (a very good description of this kind of simulators can be found in [20]).

The idea of creating one process (thread) by cell is interesting because in a real network all cells work in parallel. This approach is valid for small networks (less than 16 MICTREE cells), while for a network larger than 16 cells the operating system is not longer able to manage the problem. Most of the CPU time is used to change and store the context of each process, implying a diminution of the time allocated to the simulation.

It was difficult to establish a compromise between the number of threads and efficiency of the simulation. After many different test with different configurations, we opted for one process for each row of cells. This approach does not correspond to the reality of the network, but it does not generate any discrepancies between the results of the real network and those of the simulated network.

Having defined the architecture of the simulation engine, we had to model the network of MICTREE cells. To do so, we had to answer two questions: what do we want to observe, and how will we interact with the network?

We want to be able to observe the state of the MICTREE cell, that is, the contents of the registers $(X, Y$, and $R E G)$, the address display, the state (alive or not) of each cell, and all the input and output signals. In fact we want to be able to observe every aspect of 
an actual cell, as if we were interacting with an actual network. In addition, we wanted to observe the routing table of the cell, be able to change the clock frequency, realize a step-by-step simulation, observe the current instruction being executed in any cell, and introduce initial values (through the border cells at the edge of the network.)

To meet the above specifications, we needed two different types of cells: a MICTREE cell and an input/output cell, the last to be used as an interface with the network. By using this interface we will be able to introduce or read different values through the input/output buses (of the border cells at the edge of the network).

\subsubsection{MICTREE model}

In designing our MICTREE cell model, we tried to remain as close as possible to the reality, as described in [46]. However, in order to simplify the MICTREE model implementation we supposed that the following connections are established by default:

$\Rightarrow C K$, the clock signal;

$\Rightarrow C L R$, the reset signal;

$\Rightarrow D I N$, the input signal used to transfer and propagate the genome;

$\Rightarrow W X, X, S Y, Y$, the coordinate signals;

$\Rightarrow B Y P$, the bypass signal.

This simplification implies that we do not need to be aware of how these signals are connected: the connections will be established automatically when the cells are put together (Figure 6.3). This simplification does not generate any discrepancies between the actual network and the simulated network.

Our cell model (Figure 6.4) consists of two main parts: the first describes the physical components of the actual cell, and the second describes the virtual components necessary to the simulation.

The physical components are:

$\therefore$ A complete copy of the genome;

$\Rightarrow$ The program counter $(P C)$;

$\Rightarrow$ The 16-bit instruction register $(I R)$;

$\Rightarrow$ The router;

$\Rightarrow$ The 4-bit user register $(R E G)$; 


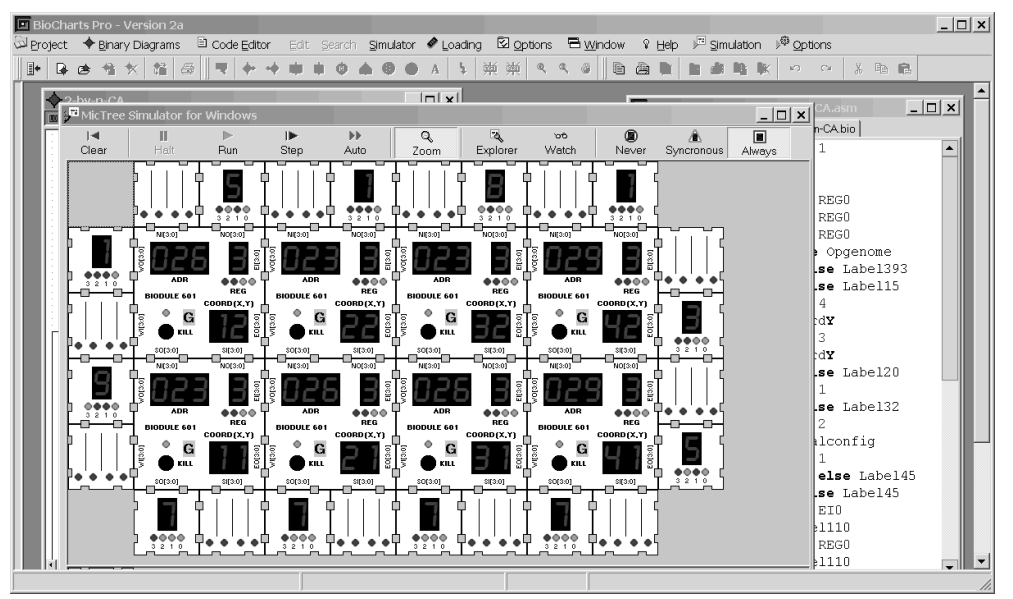

Figure 6.3: Simulation view of a 2-by-4 MICTREE network; the default connections between the MICTREE cells can be seen.

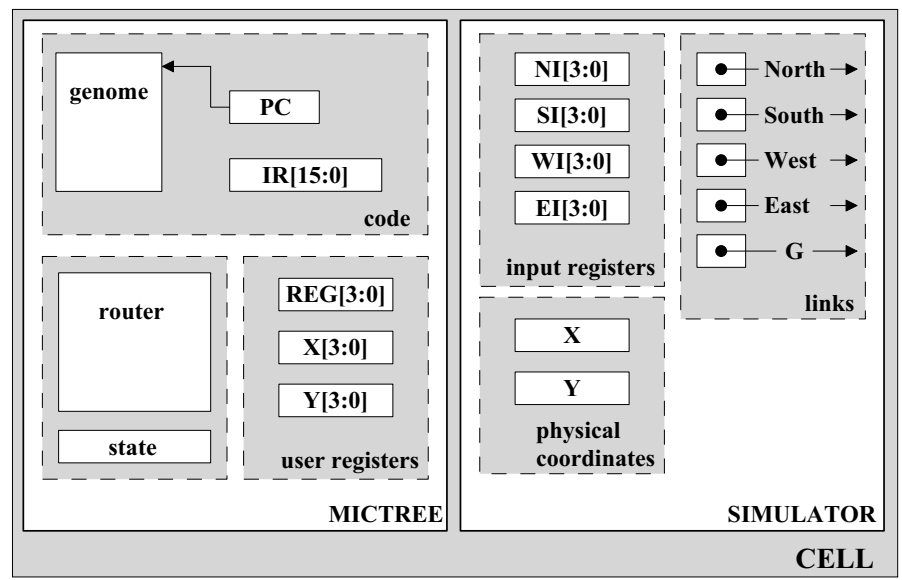

Figure 6.4: MICTREE model. The model is composed of two main parts: the left part describes the physical components of the cell, and the right part describes the virtual components necessary to the simulation.

$\Rightarrow$ The 4-bit coordinates registers $(X, Y)$.

The virtual components are:

$\Rightarrow$ the registers $(N I, S I, E I, W I)$ that store the cell input values before execution of the instructions;

$\Rightarrow$ the links towards the neighbor cells, which can be either MICTREE cells or input/output cells;

$\Rightarrow$ a link to model the global clock signal $G$;

$\Rightarrow$ the variables $X$ and $Y$, used to store the position of the cell in the memory array. 
The execution of one instruction requires four clock cycles (as in the real MICTREE cell). The input registers are loaded with the input values at the first clock edge, and the execution of the instruction is really effected at the fourth clock edge.

\subsubsection{Input/output cell model}

The input cells can be used to introduce initial values at the edge of the network or as external inputs. An input cell provides a 4-bit bus, where each bit can be programmed independently as a periodic or non-periodic external signal. If a bit is programed as a non-periodic signal, the user can change the value of this signal with a simple mouse click at simulation time. If it is a periodic signal (such a clock signal), the user can specify the period as a multiple of the system clock.

As the $G$ input of the MICTREE cell can be programmed to receive a signal coming from an external input cell, we can use an input cell to generate and feed the network with the global clock signal $G$.

The output cells can display the information coming through their 4-bit busses, and are normally connected at the edge of the network. We model this kind of cell as a 4-bit register.

\subsubsection{Cellular network model}

The network is composed of MICTREE cells and input/output cells placed at the edge of the network. To model this architecture we use two arrays:

$\Rightarrow$ a two-dimensional array of MICTREE and input/output cells, and

$\Rightarrow$ a one-dimensional array for the additional input cells (the global clock signal $G$ in the case of typical simulations).

\subsubsection{MIC Sim implementation}

The MIC Sim tool has two main components: the simulator and the graphical interface. In this section we present only the architecture of the simulator, which, in a simplified way, can be decomposed into five main modules (Figure 6.5):

$\Leftrightarrow$ the MICTREE module, implementing the MICTREE cell;

$\Leftrightarrow$ the IO module, implementing the input/output cells;

$\Rightarrow$ the CellularNetwork module, implementing a MICTREE network; 
$\Rightarrow$ the Engine module, implementing the simulation engine;

$\Rightarrow$ the Simulation module, implementing the cellular network simulator.

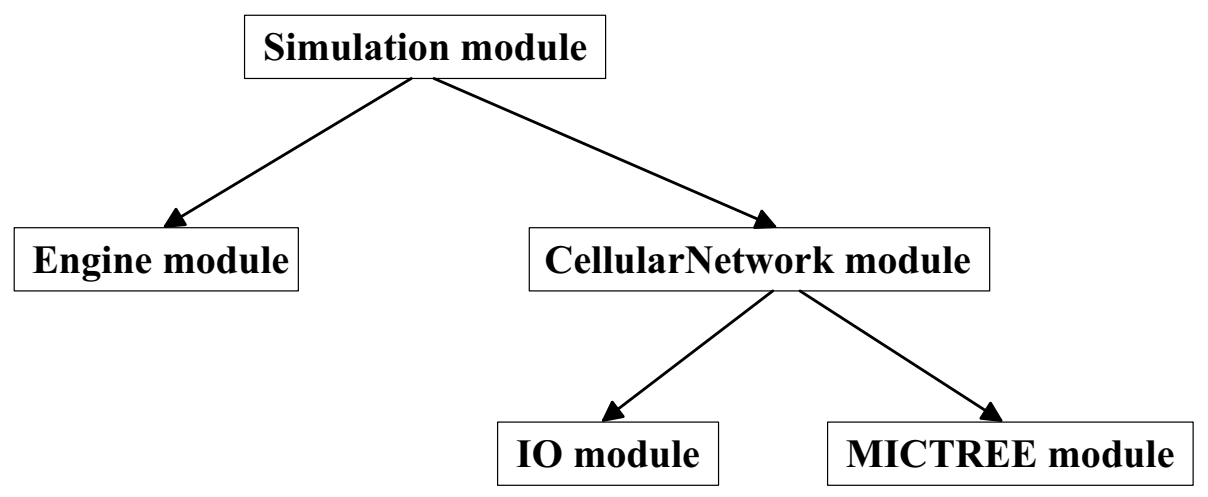

Figure 6.5: Simulator architecture.

In the IO module we define the classes TInputCell, and TOutputCell, which are used to create the InputCell, and OutputCell objects placed at the edge of the network to be simulated. The InputCell and OutputCell objects have variables used to model their behavior, and methods to access or modify these variables.

An OutputCell object is very simple: it consists simply of a 4-bit register. An InputCell object has an array of four elements of the type TInputBit, which has the following variables:

$\Rightarrow$ Val, containing the current value of the bit;

$\Rightarrow$ InitVal, containing the initial value of the bit (set a the beginning of the simulation);

$\Rightarrow$ Etype, indicating the type of signal (periodic or non-periodic);

$\Rightarrow$ Period, used to specify the clock period if the bit is used as a periodic signal;

$\Rightarrow$ Label, a string used to specify a label for the bit.

In the MICTREE module we define the class TCell, which is used to create the MICTREE objects composing the network to be simulated. A MICTREE object has variables used to model the cell's behavior, and methods to access or modify these variables. The following are some of the variables used by a MICTREE object:

$\Rightarrow P C$ (program counter) contains the address of the instruction to be executed;

$\Rightarrow$ Phys $X$ and Phys $Y$, contain the physical coordinates of the cell in the memory array modeling the network; 
$\Rightarrow X$ and $Y$ contain the coordinates computed by the genome;

$\Leftrightarrow I R$ (instruction register) contains the instruction to be executed by the cell;

$\Rightarrow$ Genome contains a complete copy of the genome microprogram;

$\Rightarrow$ Router, a two-dimensional array, implements the routing system of the cell;

$\Rightarrow$ CellState contains the state of the cell (Alive, Loading, Cleared, Waiting_Genome);

$\Rightarrow R E G$ is the state register of the cell;

$\Rightarrow N I, W I, S I$ and $E I$ are registers containing the information coming through the north, west, south, and east input busses of the cell, respectively;

$\Rightarrow$ North, West, South and East are links towards the north, west, south, and east neighbor cells, respectively;

$\Rightarrow W X$ and $S Y$ are registers containing the information coming through the west and south inputs of the cell, respectively;

$\Rightarrow$ NICell, WICell, SICell and EICell are links towards InputCells (used only if the cell is at the edge of the network);

$\Rightarrow$ NOCell, WOCell, SOCell and EOCell are links towards OutputCells (used only if the cell is at the network edge).

In the CellularNetwork module we define the class TCellularNet, which is used to create the CellularNetwork object. This object is the actual network to be simulated. A CellularNetwork object has variables used to model the network's behavior and methods to access or modify these variables. The following are some of the variables used by a CellularNetwork object:

$\Rightarrow$ Net is a two-dimensional array of TCell objects;

$\Rightarrow$ Width and Height define the network size;

$\Rightarrow$ AdditionalIN is a one-dimensional array of TInputCell objects; in typical networks this array has only one element, which is used to feed the $G$ signal into the network;

$\Rightarrow$ GenomeSrc contains a copy of the genome microprogram to be sent to the cells at loading time;

$\Rightarrow$ GenomeName and GenomeSize are the name and the size in bytes of the genome microprogram. 
In the Engine module we define the class TEngine, which implements the simulation engine. A detailed description of this module can be found in [20].

In the Simulation module we define the class TSimulation, used to create the NetworkSimulator object. This object controls all the simulation process: starting the simulation engine, initializing the thread unit, creating a thread for each row of cells, generating the system clock $(C K)$, controlling the interactions with the user, and interacting with the graphics interface (not described here).

\subsection{Visual MICTREE design environment}

In order to achieve versatility and user-friendliness, we decided to integrate all the MICTREE's software tools into a single graphical design environment code-named Visual MICTREE. This design environment consists of the MIC Editor program, the MIC Compiler, the Visual BDD program, the MICTREE Sim simulator, and a serial loader used to configure the MICTREE network through the computer's serial port.

The microcode executed by the MICTREE binary decision machine can be generated by compiling the MIC code written with the MIC Editor (or another text editor), or, alternatively (and more simply), by compiling the MIC code generated by Visual BDD. This microcode can then be sent to the MICTREE network through the computer's serial port by using the loader, starting the "life cycle" of our artificial organism.

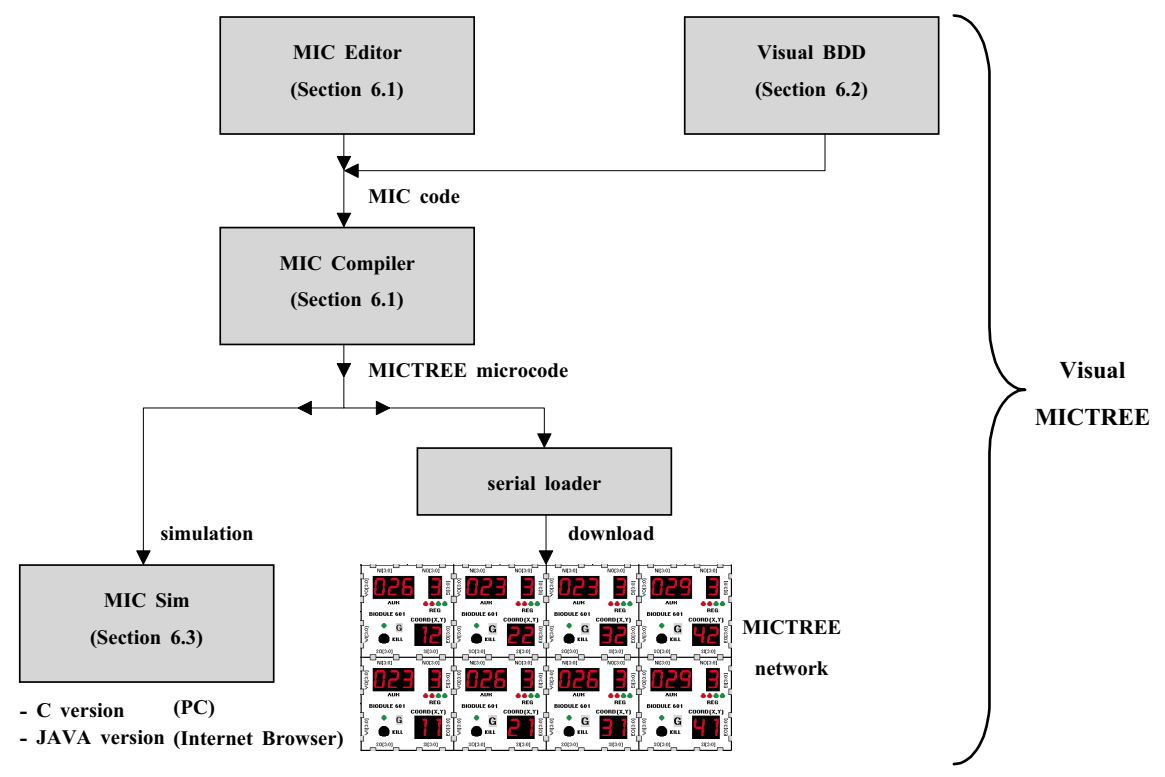

Figure 6.6: Development cycle with the Visual MICTREE design environment.

Figure 6.6 shows the development cycle with the Visual MICTREE design environment. This development cycle will be used in the next section, were we develop the 2-by-n hybrid cellular automaton example. 


\subsection{2-by-n hybrid cellular automaton example}

In this section we implement, using the Visual MICTREE design environment, the 2-by-n hybrid cellular automaton example of Section 3.4. We will not provide all the details of the procedure, but rather limit ourselves to the main steps. A complete description of the use of the Visual MICTREE design environment can be found in [65] and in [116,117] the details of the MIC Sim component are described.

The top-down design cycle begins with the description of the high-level flowchart, defining the complete genome microprogram (Figure 6.7). The next step is to describe the flowcharts associated with the CoordX, CoordY, Localconfig, and Opgenome subprograms, as shown in Figure 6.2. The software can support as many hierarchical design views as needed.

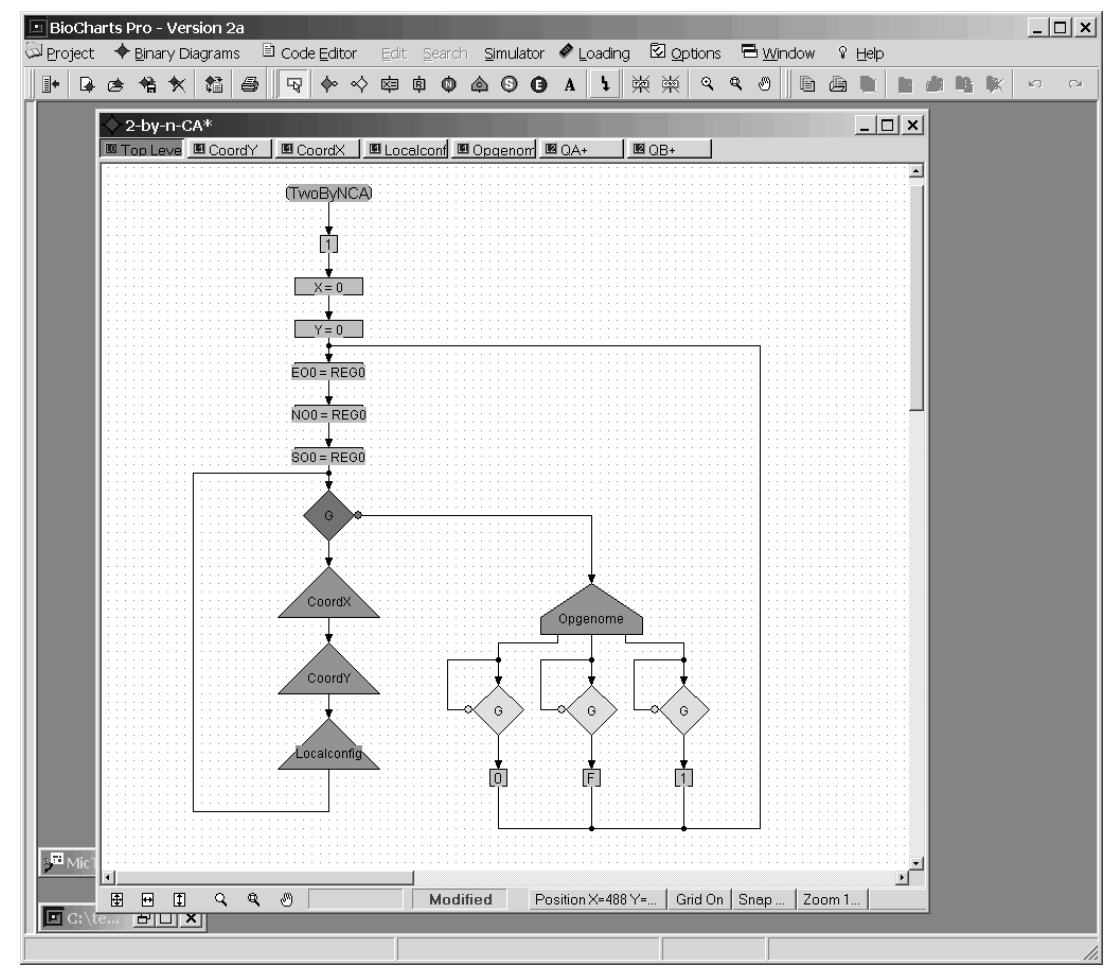

Figure 6.7: 2-by-n hybrid cellular automaton high-level flowchart.

Once the genome microprogram has been described, we can generate the code using the MIC code generator. If no errors are present in the graphical description, the MIC editor window, containing the mnemonic microprogram, appears (Figure 6.8a). Otherwise, a dialog box with the corresponding error code is displayed (Figure 6.8b).

After the mnemonic microprogram generation, we can compile the code using the MIC compiler. If the compilation process is successful, the MIC Compiler displays a dialog box indicating that no compilation errors were detected (Figure 6.9a). Otherwise, 


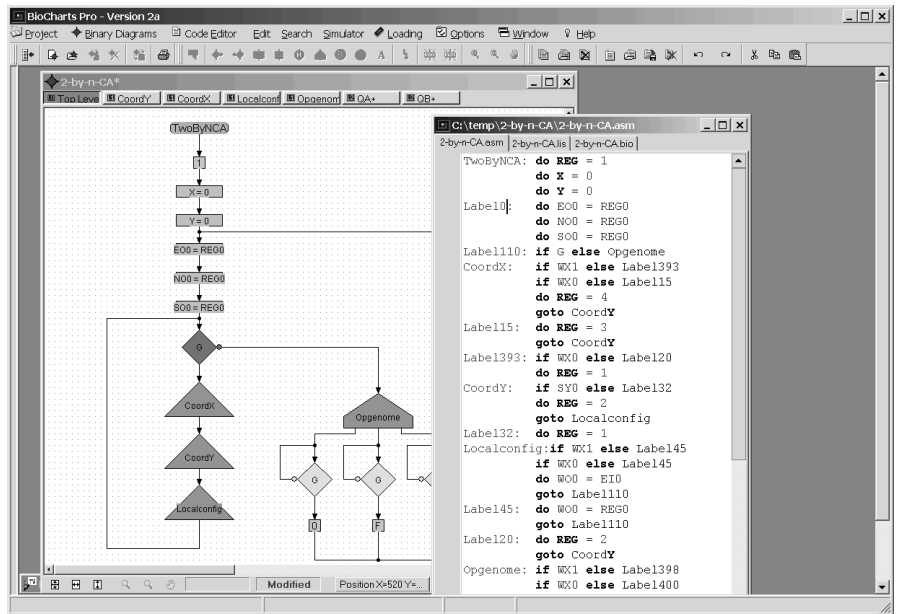

(a)

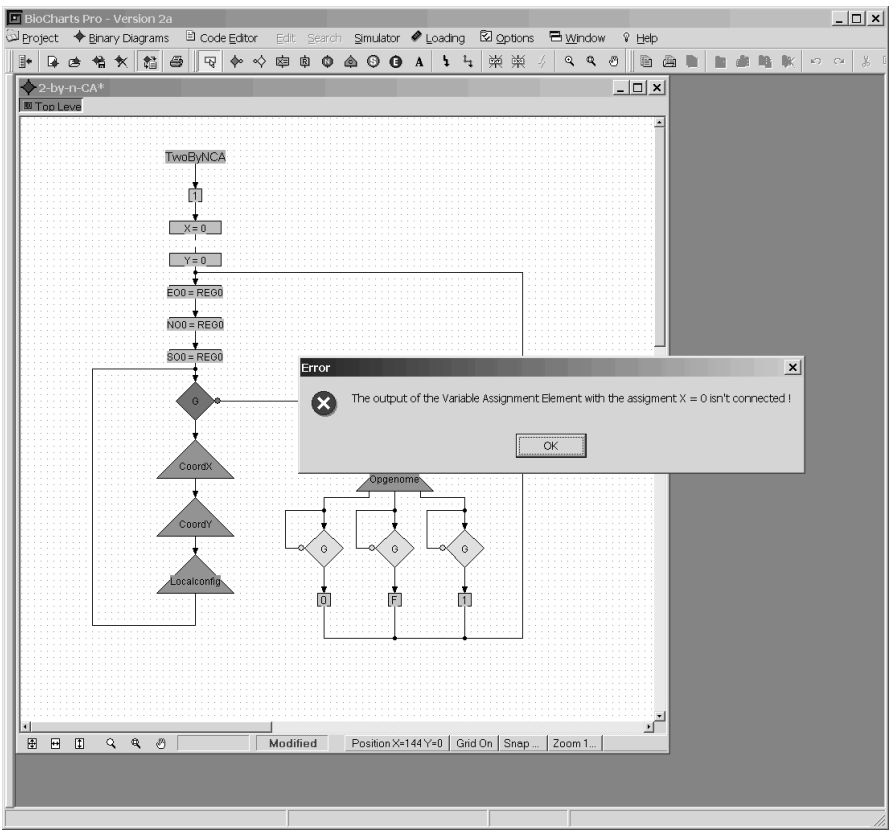

(b)

Figure 6.8: Mnemonic microprogram generation. (a) The microprogram generation process was successful, and the MIC editor window with the generated microprogram is displayed. (b) An error occurred, and a dialog box describing the error is generated. 
the MIC Compiler displays a dialog box indicating the number of compilation errors. A new text panel, with the error line number and the kind of error, is added at the bottom of the code editor window (Figure 6.9b). By double-clicking the error message, the line containing the error into the code editor window is highlighted.

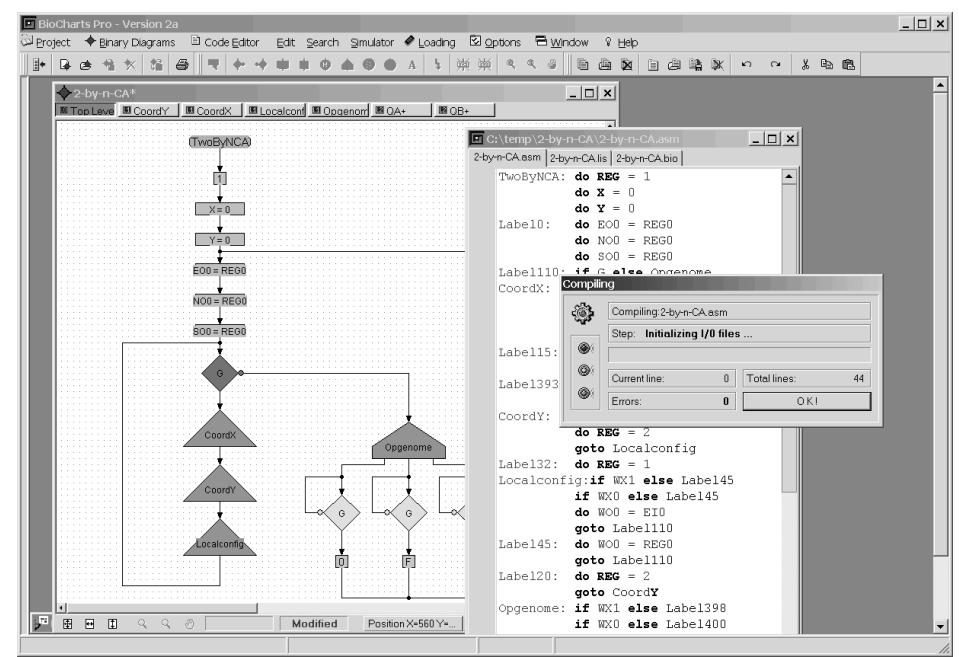

(a)

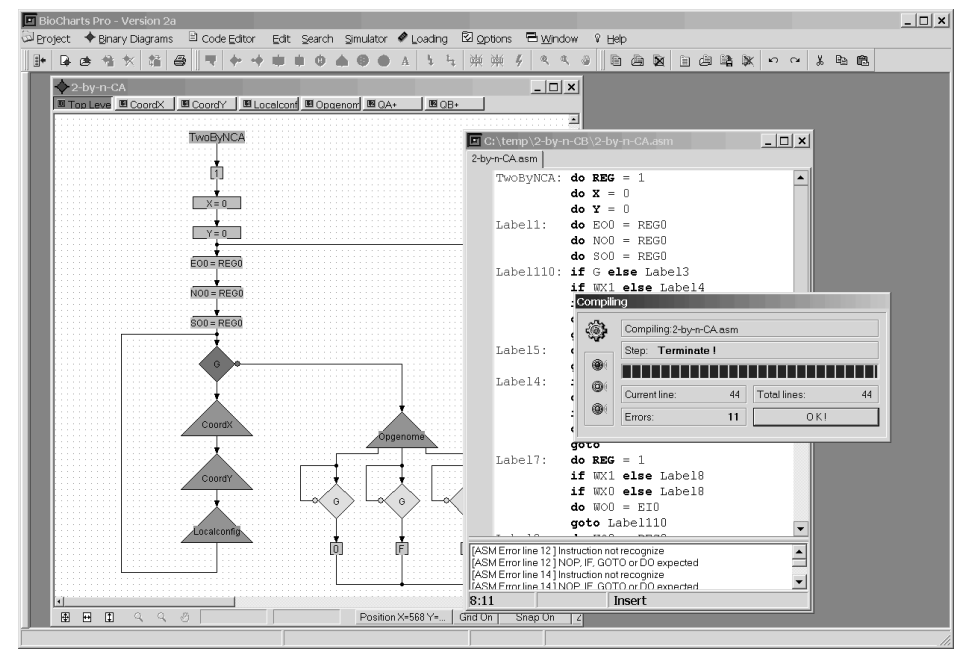

(b)

Figure 6.9: Compilation. (a) The compilation process was successful, and the MIC Compiler displays a dialog box indicating that no compilation errors were detected. (b) An error occurred, the MIC compiler displays a dialog box indicating the number of compilation errors.

Once all the errors have been corrected and the compilation process is finished, we can start the simulation of our artificial organism. When we launch the simulator, a dialog box is displayed, prompting for the size of the network to be simulated (Figure 6.10). In this simulation, the genome that we had already generated will be used by default. 


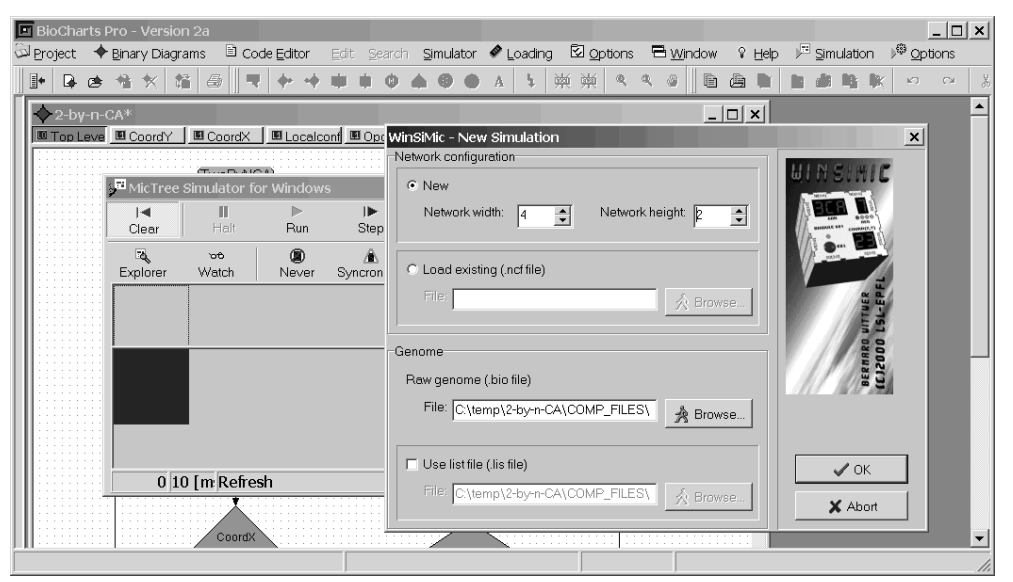

Figure 6.10: Starting the MIC Sim simulator.

The next step is to generate the global clock signal $G$ using an external input module, by connecting its $G$ output signal to each $G$ input of the MICTREE cells. The genome can then be loaded into the network by clicking the RUN button of the MIC Sim window. Clicking again the RUN button starts the simulation (Figure 6.11).

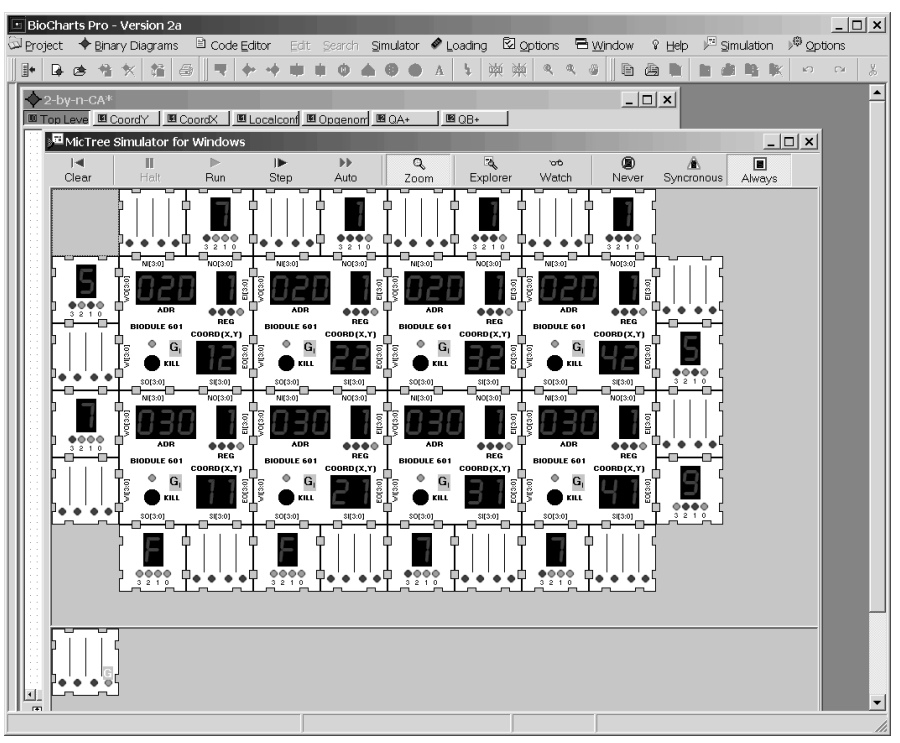

Figure 6.11: Starting the MICTREE network simulation.

During the simulation, the user can interact with the interface and modify or access the state of any MICTREE cell. Figure 6.12 shows the simulator window and the object explorer window. Interacting with the object explorer, the user can change or visualize information about the selected object (in this case, the shadowed MICTREE cell at the left-top corner of the network). We can appreciate, from this figure, that the user can 


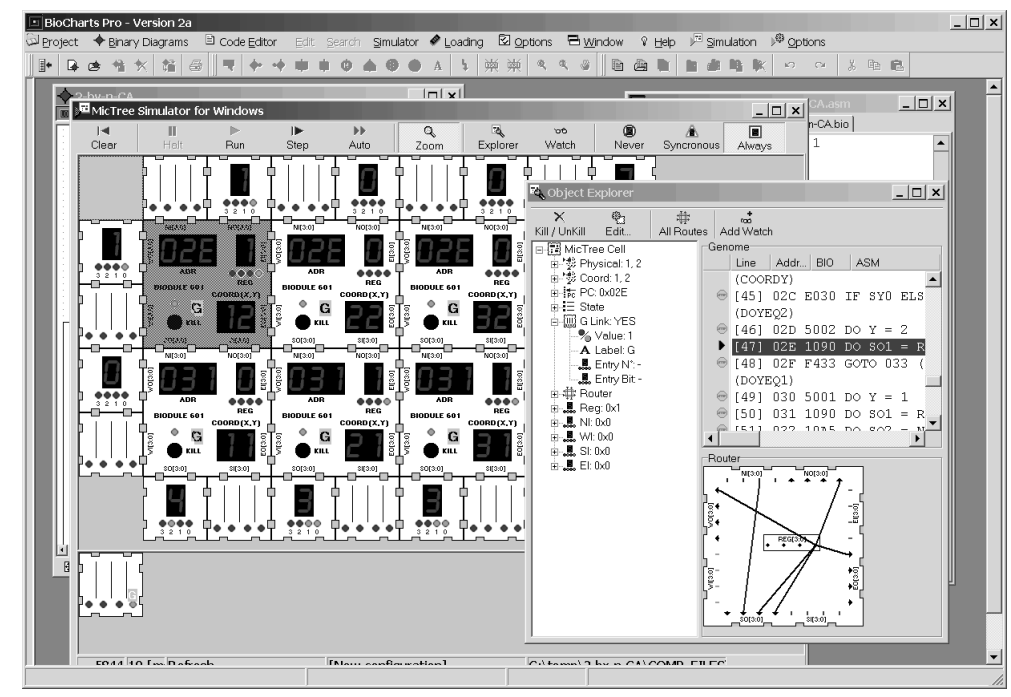

Figure 6.12: MICTREE network simulation window (at the left) and the object explorer window (at the right) associated with the shadowed MICTREE cell at the left-top corner of the network. We can appreciate the default connections and the border input/output cells, as the additional input cell feeding the network with the $\mathrm{G}$ signal (at the left-bottom corner of the simulation window). $\mathrm{G}$

visualize the routing path of each MICTREE cell. This functionality is very important to verify the correctness of the corresponding part of the genome microprogram.

After verifying that the artificial organism behaves in the expected way, we are ready to configure a real MICTREE network. The genome microcode can then be sent to the MICTREE network through the computer's serial port using the loader component of the Visual MICTREE design environment. Figure 6.13 shows the loader window, which allows us to select the serial port to be used. If an error occurs, a message is displayed in the left-bottom text panel of the loader window.

\subsection{Conclusion}

In this chapter we presented the features and the software architecture of the tools designed to deal with the programming complexity of implementing artificial organisms in the Embryonics architecture. We showed, through the example of Section 6.5, the main steps for the edition, simulation, and configuration of an actual network implementing an artificial organism.

We integrated all the software components (MIC Editor, MIC Compiler, Visual BDD, MIC Sim, and the serial loader) into a single graphical design environment in order 


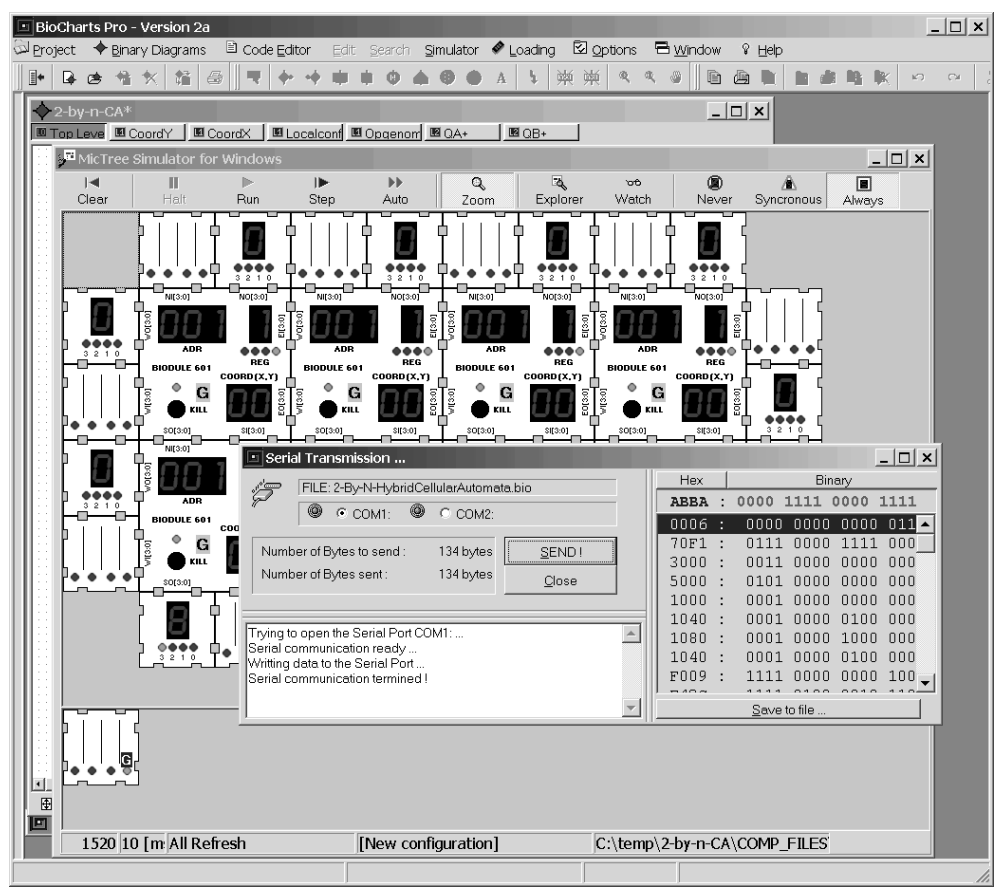

Figure 6.13: Loading the real MICTREE network.

to achieve versatility and user-friendliness. A Java version of the MIC Sim simulator was developed to provide the user with a stand-alone simulation via an Internet browser [10].

The software tools were designed taking into account the possibilities of evolution of the MICTREE cell architecture (for example more memory and larger internal registers, wider input/output busses). The resulting object oriented software architecture is easily scalable to meet all these constraint through minor changes to the code.

The MIC Sim simulator provides the user with debugging capabilities not present in a real MICTREE network, such as a watching window to display the state of different internal variables of any MICTREE cells, the routing table of a MICTREE cell, the visualization of the current instruction being executed by a particular MICTREE cell, the possibility of displaying, in a external output module, information coming from any bit of any MICTREE cell in the network, a step-by-step simulation, the execution of $n$ clock cycles, etc. 


\section{Chapter 7}

\section{Conclusions}

In this work we presented a novel automaton, truly "multicellular" in the sense that every cell contains a complete copy of the genome. We have shown that such a multicellular automaton is able to self-repair and to self-replicate. We then showed that it is possible to embed a universal Turing machine into our automaton, obtaining a self-replicating universal Turing machine, one of the ultimate dreams of John von Neumann.

The mapping of the universal Turing machine onto our multicellular array was made possible through the introduction of a modified version of the W-machine, i.e., an interpreter of the PICOPASCAL language. We showed that an ideal architecture was able to deal with applications of any complexity, i.e., with a semi-infinite data tape. We also presented an actual implementation in which we relaxed somewhat the requirements of the ideal architecture in order to use a smaller number of our MICTREE artificial cells. We slightly simplified our implementation by presenting the example of the binary counter in which the data are binary-coded and where the direction of the head's motion coincides with the internal state (in the general case, functions $Q+$ and $D+$ are independent).

The property of universal construction raises issues of a different nature, since it requires (according to von Neumann) that a MICTREE cell be able to implement organisms of any dimension. This challenge can be met by decomposing a cell into molecules and tailoring the structure of cells to the requirements of a given application $[52,55]$.

We presented the features and the software architecture of the tools designed to deal with the programming complexity of implementing artificial organisms in the Embryonics architecture. We showed, through the example of Section 6.5, the main steps for the design, simulation, and configuration of a real network implementing an artificial organism.

Finally we integrated all the software components (MIC Editor, MIC Compiler, Visual BDD, MIC Sim, and the serial loader) into a single graphical design environment in order to achieve versatility and user-friendliness. A Java version of the MIC simulator was developed to provide the user with a stand-alone simulation via an Internet browser [10]. 


\subsection{Original contributions}

When faced with a thesis such as this one, describing a research effort which is closely integrated within a larger project, it is sometimes difficult to precisely identify the original contributions of the author. In this section I will try to point out my personal contributions to the project.

The first contribution consisted in the generalization of a synthesis methodology for the implementation of complex applications on Embryonics machines. Using existing and original examples (notably the 2-by-n hybrid cellular automaton described in Section 3.4), we proposed a top-down design methodology based on the following processes:

$\Rightarrow$ the decomposition of the complete genome microprogram (the artificial genome) into a set of smaller sub-microprograms;

$\Rightarrow$ the systematic construction and optimization of these sub-microprograms thanks to the utilization of binary decision trees and diagrams;

$\Rightarrow$ the rigorous organization of these sub-microprograms conforming to the Embryonics architecture (computation of the reset conditions and of the global configurations, of the $X$ and $Y$ coordinates, of the initial states and local configurations as a function of the $X$ and $Y$ coordinates, and finally computation of the genome's operational part describing, according to the coordinates, each gene of the artificial organism).

One of the main goals of the Embryonics project was to determine if, given modern technology, Von Neumann's dream of a self-replicating machine can be realized in hardware. The main contribution of this thesis was to demonstrate that such a dream was possible, thanks to the Embryonic architecture, in particular to the MICTREE cell.

When we first approached the problem, the state of the art for self-replicating machines exhibiting universal computation was represented by software implementations. Thus, inspired by von Neumann ideas and using the Embryonics architecture, we developed in Chapter 5 all the details of the architecture implementing an ideal multicellular universal Turing machine with self-repairing and self-replicating capabilities, that is, exhibiting universal computation.

The number of MICTREE cells being limited, we were forced to introduce some modifications to the ideal architecture described earlier. Our final architecture consists of six rows $(Y=1 \ldots 3)$ and ten columns $(X=1 \ldots 9,9)$ of MICTREE cells (Figure 5.38). Thus, given a sufficiently large space, the self-replication process can be repeated for any number of specimens in the $Y$ axis, while the self-repair process on the $X$ axis is only limited by the number of spare columns (one in our actual implementation).

This implementation required considerable original thought since, to the best of our knowledge, it constitutes the first hardware implementation of such a machine. 
Chapter 6 contains the third major contribution of this thesis, the implementation of all the software tools developed to deal with the programming complexity of designing artificial organisms with the MICTREE cells, as well as, the object model of the cell. All these software tools were integrated into a single graphical design environment running on a PC.

The software tools were designed taking into account the possibilities of evolution of the MICTREE cell architecture (for example, more memory and larger internal registers, wider input/output busses, a different routing system). The resulting object-oriented software architecture is easily scalable to meet all these constraints through minor changes to the code. It can also be easily modified for other artificial cell architectures.

One of the most important components is the MIC Sim tool that provides the user with debugging capabilities not present in a real MICTREE network, such as a watching window to display the state of different internal variables of any MICTREE cells, the routing table of a MICTREE cell, the visualization of the current instruction being executed by a particular MICTREE cell, the possibility of displaying, in a external output module, information coming from any bit of any MICTREE cell in the network, a step-by-step simulation, the execution of $n$ clock cycles, etc.

\subsection{Problems and issues}

During the development of the present work some technical problems appeared. We will mention only the two most important.

The first problem was the distribution of the $C K$ clock signal for networks composed of five or more columns of cells. In such a case, the cells placed in the last columns of the network behaved in a completely asynchronous way, making the behavior of the artificial organism completely chaotic.

This problem was very difficult to solve, because detailed measurements seemed to indicate that the genome microprogram was the source of the problem. In fact the problem was a degradation of the clock signal, which is not regenerated when it is propagated going from one cell to the next. The MIC Sim simulator was very helpful when looking for a solution to this problem.

The adopted solution consisted of: (1) regenerating the clock signal every four columns and (2) making the distribution of this signal symmetrical. As a precaution, we adopted the same measure with the global clock signal $G$.

The second problem was the detection of faults in the routing system of the MICTREE cells. In fact, when working with large arrays, we supposed (naively) that all the MICTREE cell would be fault-free. As a consequence, we never considerate that the cells themselves, and not the genome microprogram, might be the source of the problems we encountered. 
Faults in the routing system are difficult to find. A broken wire, for example, will only be detected when we use it as part of our routing. When signals are routed through two or more cells, how can we detect which cell on the path is the faulty one? In order to solve this problem, we developed some test programs that allow us to verify the operation of the cells system. These tests were applied to each MICTREE cell to be used for the implementation of an artificial organism. The MIC Sim simulator tool was again very helpful in finding a solution to this problem.

A more appropriate solution would consist of realizing a self-diagnostic test on the network to be used for the implementation of the artificial organism. Or, even better, to embed the self-diagnostic program into the genome microprogram. Unfortunately, lack of time did not allow us to realize any research in this field. Very interesting works in this area do however exist $[72,82,115]$.

\subsection{The future of Embryonics}

This thesis represents a step forward in the realization of the Embryonics project. However, it does not by any means represent a closure for the project as a whole, or even for the development of the ontogenetic axis. Research is continuing along all three axes of the POE model (SubSection 1.2.2).

Along the phylogenetic axis, where the design of the Firefly machine demonstrated the feasibility of hardware evolution, we are currently studying the application of evolutionary strategies to the design of hardware systems such as, for example, fuzzy controllers. As for future developments of evolutive hardware systems, we are investigating the feasibility of open-ended undirected evolutionary strategies, that is, systems that evolve not towards a precise, user-defined goal, but independently. Such an approach is undoubtedly a much closer approximation of natural evolution.

The epigenetic axis is advancing into the application phase, in wich we are trying to apply our algorithms to the solution of real-life problems and for the control of autonomous robots. An interesting possible advance along this axis would be the creation of neural networks capable of continuous learning, that is, of learning new behaviors (and consequently of adapting their structure) not only during a dedicated learning phase, but also while operating. Obviously, such systems would much more closely approach the behavior of biological neural networks than conventional ANNs do.

On the ontogenetic axis, to which this thesis belongs, even if a considerable amount of work remains, we can begin to glimpse a possible closure. The next major step in the development of this axis is the design of the BioWall, an extremely complex machine which is an undergoing project in our laboratory. The BioWall is intended as a reconfigurable computing tissue capable of interacting with its environment by means of a large number of touch-sensitive elements coupled with LED displays. The final implementation will have the respectable dimensions of approximatively $5.5 \times 0.9 \mathrm{~m}$. The entire 
tissue contains 3200 molecules, each consisting of one two-color $8 \times 8$ dot LED display, one transparent touch-sensitive element, and one reconfigurable circuit (a Xilinx Spartan XCS10XL FPGA). The display and the transparent touch-sensitive element are physically joined by an adhesive film. Each molecule is interconnected with its four direct neighbors. The entire BioWall consists of about 32 mio. reconfigurable gates, 3200 binary inputs, and more than 50'000 bi-color outputs. Figure 7.1 shows the current BioWall prototype, made up of about 2000 molecules [98, 105].

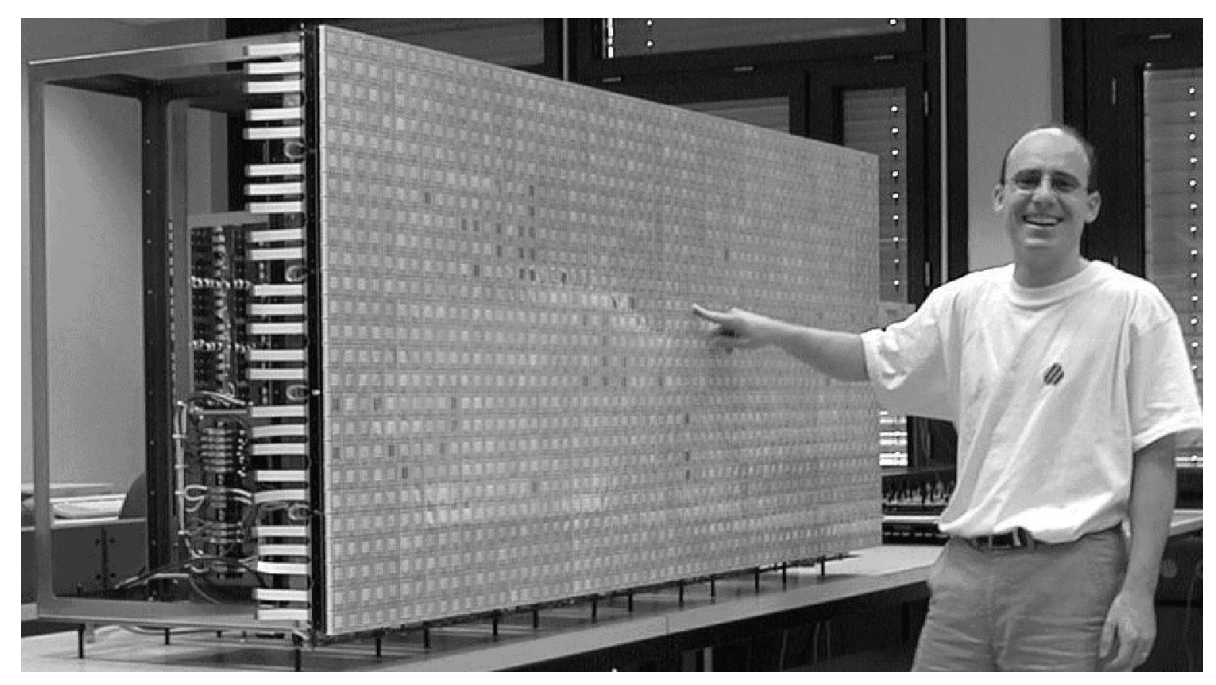

Figure 7.1: On the left, the current BioWall prototype, consisting of about 2'000 molecules. On the right, Christof Teuscher, one of the conceptors. Photo André Badertscher.

\subsubsection{The BioWatch on the BioWall's computing substrate}

In our laboratory, the next major step in the Embryonics project is the design of the BioWatch, a complex machine which will be presented on the occasion of a cultural event which will soon take place in Switzerland. The function of the machine will be that of a self-repairing and self-replicating watch, counting seconds, minutes and hours. The implementation of the watch will take place in a reconfigurable tissue, the BioWall $[58,59]$. A computer simulation of the BioWatch application is depicted in Figure 7.2.

Each of the six digits required to count the hours, minutes, and seconds is represented thanks to an Embryonics cell built with 400 Embryonics molecules, each molecule corresponding to the BioWall's fundamental element. In addition to the six cells, two spare cells are added to the right of the wall (Figure 7.2). Each cell also contains several spare columns of molecules [59,98, 105]. 


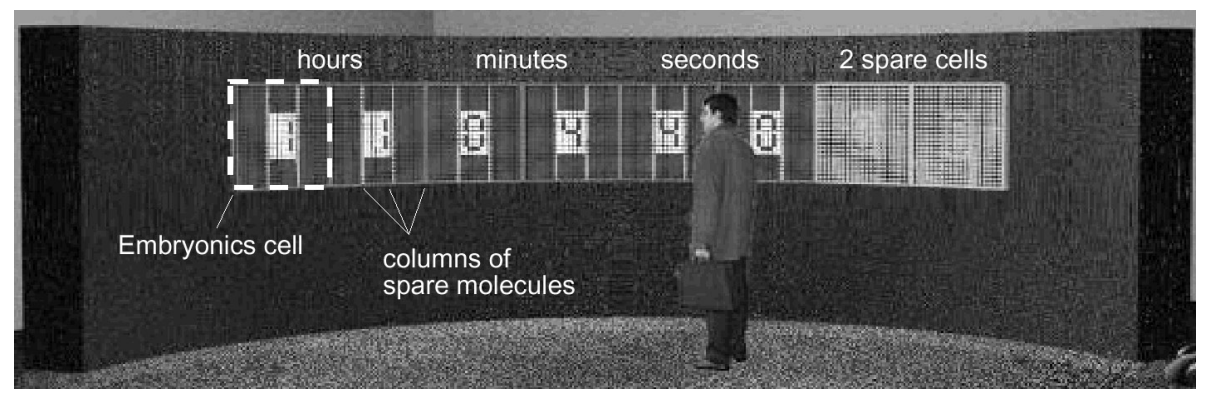

Figure 7.2: A computer simulation of the BioWatch, a self-repairable and selfrepairing large-scale watch using the BioWall's reconfigurable computing substrate.

\subsubsection{Towards Bio-Inspired Computing Tissues}

A bio-inspired reconfigurable computing tissue [58] is a completely homogeneous twodimensional surface, built up of simple, locally-interconnected basic elements (also called molecules). Each molecule consists of at least one input, one output, and one reconfigurable computing unit (typically, an FPGA). Often, inputs, outputs, and computing units are organized in three hierarchical layers. However, the layers might also be unified into a single physical substrate. The substrate might be rigid, flexible, organic, inorganic, etc. The molecule's physical size can range from a nano-electronic structure to a largescale object. A molecule's inputs and outputs might include temperature sensors, force sensors, microphones, cameras, motors, speakers, displays, etc. The molecule's operation is determined by a configuration bitstream or artificial genome. Both deterministic and non-deterministic genotype to phenotype mappings are imaginable. The system has a completely molecular structure that is scalable without any architectural limits. Molecules are simple and compute in parallel. Any computation is purely local since there are no global interconnection lines and no centralized control $[98,105]$.

The concept of bio-inspired and reconfigurable computing tissues is new and promising. The idea clearly goes beyond a simple assembly of input, output, and reconfigurable computing elements. Recent research in display technology and organic electronics (e.g., [22]) confirms a new tendency towards intelligent, interactive, and flexible systems. To the best of our knowledge, no current approach combines in one and the same substrate a cellular, intelligent, and reconfigurable structure with embedded input and output channels.

\subsubsection{Towards POEtic Machines}

The POE model classifies bio-inspired hardware systems along three axes (phylogeny, ontogeny, and epigenesis), where the ontogenetic axis describes the Embryonics project. A natural extension which suggests itself is the combination of two and ultimately all three 
axes, in order to attain novel bio-inspired hardware. An example of the latter would be an artificial neural network (epigenetic axis), implemented on a self-replicating and selfrepairing multicellular automaton (ontogenetic axis), whose genome is subject to evolution (phylogenetic axis).

Looking (and dreaming) toward the future, one can imagine nano-scale systems becoming a reality, endowed with evolutionary, reproductive, regenerative, and learning capabilities. Such systems could give rise to novel species which will coexist alongside carbon-based organisms. Thus the design of POEtic machines that evolve, grow, and learn, constitutes, perhaps, our ultimate challenge [98, 105]. 



\section{Appendix A}

\section{Cellule MICTREE : description et utilisation}

Un biodule 601 (ou cellule MICTREE) est un logidule configurable muni de signaux de contrôle, de 16 signaux d'entrée et de 16 signaux de sortie groupés en bus de 4 bits permettant la construction facile de réseaux bidimensionnels. Ce logidule est muni d'une machine de décision binaire et d'une mémoire. Son comportement, c'est-à-dire son interaction sur les signaux de sortie, est programmé. Il est particulièrement bien adapté pour la programmation biologicielle, c'est-à-dire s'inspirant de la biologie [19]. En effet, ce logidule est capable de dicerner sa position dans un réseau et d'exécuter une partie bien spécifique du programme "distribué" dans le réseau (le génome). Cette caractéristique rend le programme très semblable aux génomes que l'on trouve chez les espèces vivantes. Chaque cellule, quel que soit son rôle, contient le même génome. Seule une partie du génome est utile à une cellule donnée pour savoir quel doit être son comportement dans l'organisme vivant. C'est l'emplacement géographique de la cellule qui détermine la partie utile du génome, c'est-à-dire sa fonction au sein du réseau.

Cette annexe a pour but d'expliquer le fonctionnement du logidule ainsi que sa programmation (génomique) à l'aide des outils développés sur MacIntosh par $\mathrm{M}$. Goeke [23]. Cette documentation est divisée en trois sections. La section A.1 est consacrée à l'interfaçage des biodules entre eux. La section A.2 traite du fonctionnement interne du biodule 601. Finalement, la section A.3 couvre le jeu d'instructions du processeur embarqué dans le biodule 601 ainsi que sa programmation. 


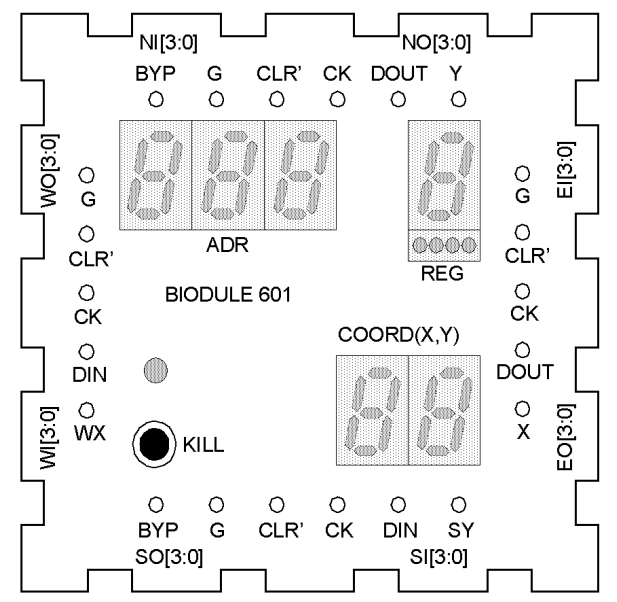

Figure A.1: Face supérieure du biodule.

\section{A.1 Interfaçage du biodule 601}

\section{A.1.1 Généralités}

Le biodule 601 se présente comme un logidule dont les connexions automatiques sont constituées de huit bus de 4 bits (NI3:0, NO3:0, EI3:0, EO3:0, SI3:0, SO3:0, WI3:0 et WO3:0). Quatre bus sont utilisés en sortie et quatre autres en entrée. Chaque côté (North, South, East et West) du logidule, comprend un bus en entrée et un autre en sortie. Sur le dessus du logidule se trouvent d'autres signaux (voir table A.1).

Le sens permet de savoir qui fournit le signal. Lorsqu'un signal est indiqué comme une entrée, c'est le monde extérieur qui le fournit au biodule. S'il est en sortie, c'est le biodule qui génère ce signal.

Pour des raisons de commodité, plusieurs signaux (tel que $C K$ par exemple) apparaissent plusieurs fois sur le logidule (figure A.2). A l'intérieur du biodule, tous ces signaux (DIN, DOUT, G, CK, CLR et BYP) sont physiquement reliés entre eux. Ainsi, une des bornes peut être utilisée en entrée et l'autre peut être vue comme une sortie. Ces signaux donnent l'impression d'être des entrées-sorties mais ne sont en fait que des entrées.

Les signaux DIN, DOUT, $X, W X, Y$ et $S Y$ sont utilisés pour la transmission de données en série au rythme de $C K$. Cette information est sérialisée et dé-sérialisée automatiquement par le biodule. DIN et DOUT servent à transmettre le génome alors que $X, W X, Y$ et $S Y$ servent à transmettre la position d'une cellule à sa voisine.

Finalement, le signal $B Y P$ est utilisé par colonne. Chaque cellule d'une colonne est reliée par ce signal à toutes ses voisines de la même colonne. Ce signal permet de rendre "invisible" une colonne du réseau. 
Table A.1: Signaux disponibles sur le biodule 601

\begin{tabular}{|c|c|c|}
\hline Non & Sens & Description \\
\hline CK & entrée & $\begin{array}{l}\text { Horloge de la machine de décision binaire du biodule et horloge de } \\
\text { synchronisation pour le chargement du programme }\end{array}$ \\
\hline CLR & entrée & $\begin{array}{l}\text { Signal de remise à zéro de l'ensemble des registres du biodule (le } \\
\text { biodule retourne dans une phase d'attente et de chargement de } \\
\text { génome) }\end{array}$ \\
\hline BYP & entrée/sortie & Permet à une cellule se suicidant de tuer l'ensemble de la colonne. \\
\hline G & entrée & Signal global (par exemple pour la synchronisation) \\
\hline DIN & entrée & Entrée du programme arrivant de la cellule voisine (génome) \\
\hline DOUT & sortie & Sortie du programme vers la cellule voisine (génome) \\
\hline WX & entrée & $\begin{array}{l}\text { Coordonnée X provenant de la cellule immédiatement à } \\
\text { gauche (West) }\end{array}$ \\
\hline $\mathrm{X}$ & sortie & Coordonnée $\mathrm{X}$ de la cellule \\
\hline SY & entrée & $\begin{array}{l}\text { Coordonnée Y provenant de la cellule immédiatement en dessous } \\
\text { (South) }\end{array}$ \\
\hline$Y$ & sortie & Coordonnée Y de la cellule \\
\hline
\end{tabular}

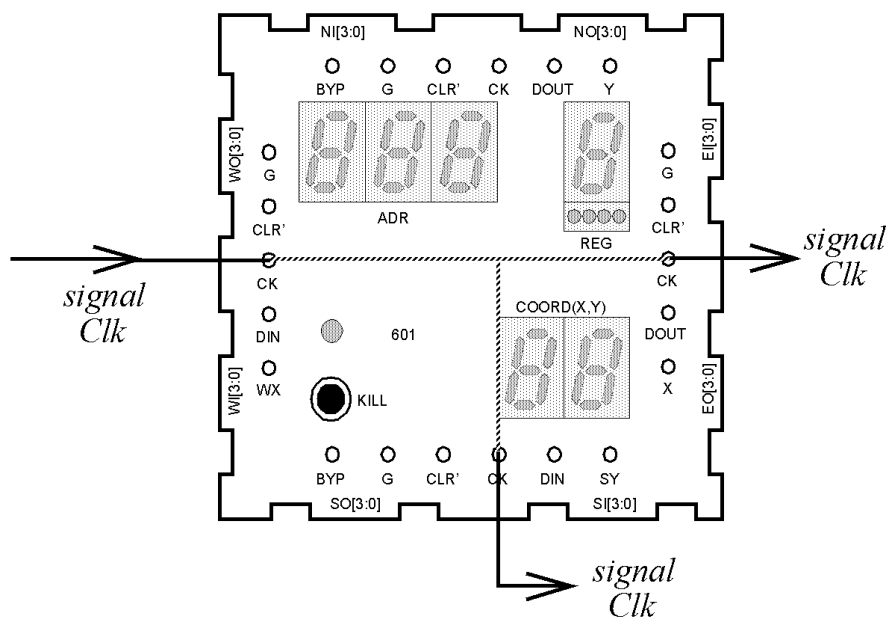

Figure A.2: Signaux apparaissent plusieurs fois sur le dessus du biodule.

Toute connexion en entrée laissée non connectée est vue comme inactive (état logique 0) par le biodule.

La face supérieure du biodule 601 (figure A.3) présente trois groupes d'affichages 7 segments. Le premier, en haut à gauche (A), composé de trois chiffres, représente l'adresse de l'instruction exécutée par le biodule. Le second, en haut à droite $(\mathrm{B})$, reflète la valeur du registre d'usage général du biodule. Sa valeur est également affichée sous forme binaire à l'aide des quatre LED (Light Emitting Diode) placées juste en dessous. Finalement, le dernier groupe, en bas à droite $(\mathrm{C})$, indique les coordonnées du biodule 


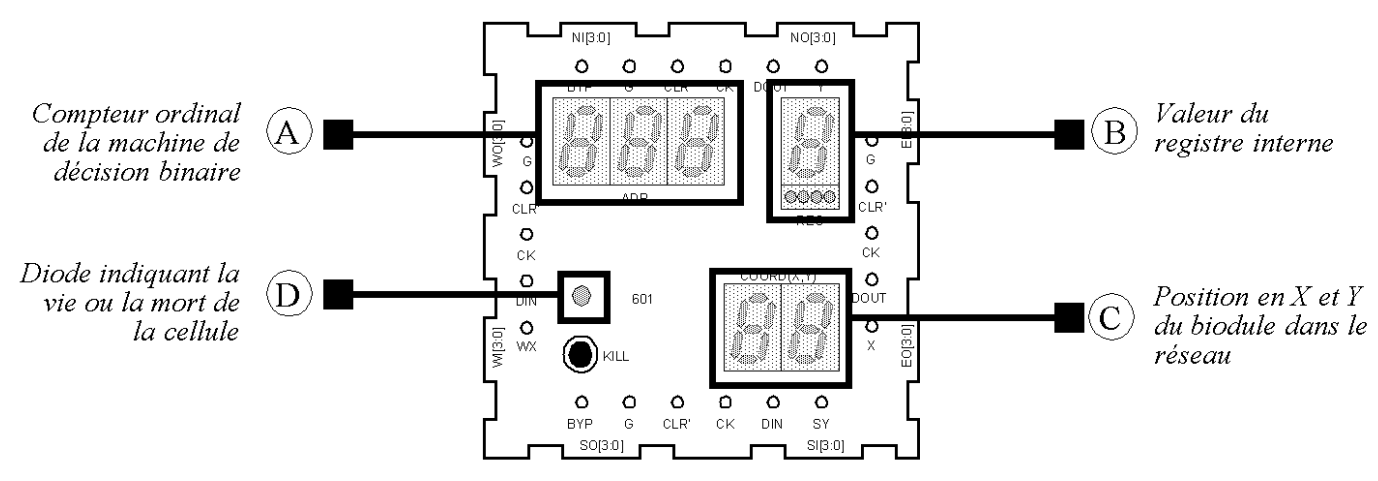

Figure A.3: Affichages du biodule 601.

dans le réseau. Le chiffre de droite indique la position en $X$ : c'est cette même valeur qui est envoyée directement à l'est par le signal $X$. La seconde valeur indique la position en $Y$; cette valeur est transmise à la cellule au nord par le signal $Y$.

Une LED (D), placée au dessus du bouton pressoir KILL indique si la cellule est vivante ou morte. Si sa couleur est verte, la cellule est vivante. Si sa couleur est rouge, la cellule est morte. Si cette LED est éteinte, la cellule est en attente de chargement du génome; c'est l'état après remise à zéro (activation du CLR).

Une cellule morte ne charge pas un nouveau génome. Elle doit être vivante pour charger un génome et passer du mode chargement au mode exécution. Pour cette raison, toutes les cellules d'un réseau doivent impérativement être vivantes (ou en attente de chargement) après la mise sous tension du réseau.

Le bouton pressoir KILL, qui fonctionne comme un commutateur, permet de tuer ou de ressusciter une cellule (ou toute une colonne si les cellules sont reliées entre elles par l'intermédiaire du signal $B Y P$ ). Ce bouton est associé à la LED bicolore évoquée plus haut (D).

\section{A.1.2 Configuration classique}

La construction d'un réseau cellulaire nécessite, outre la connexion des biodules entre eux, un câblage spécifique. Il faut en effet prévoir :

1. la distribution d'une horloge commune pour le chargement et l'exécution du programme par chaque logidule $(C K)$;

2. la distribution d'un signal de remise à zéro global (CLR);

3. la distribution du programme (génome) dans le réseau (DIN et DOUT);

4. la transmission des coordonnées entre cellules ( $W X, X, S Y$ et $Y$ ); 
5. la création de colonnes à état commun (mort ou vivant) $(B Y P)$;

6. éventuellement, la distribution d'un signal global $(G)$.

\section{A.1.3 Câblage de l'horloge $(C K)$}

L'horloge $C K$ utilisée pour synchroniser l'arrivée des données (génome) dans les biodules ainsi que pour rythmer la machine de décision binaire doit être distribuée sur tous les biodules (figure A.4).

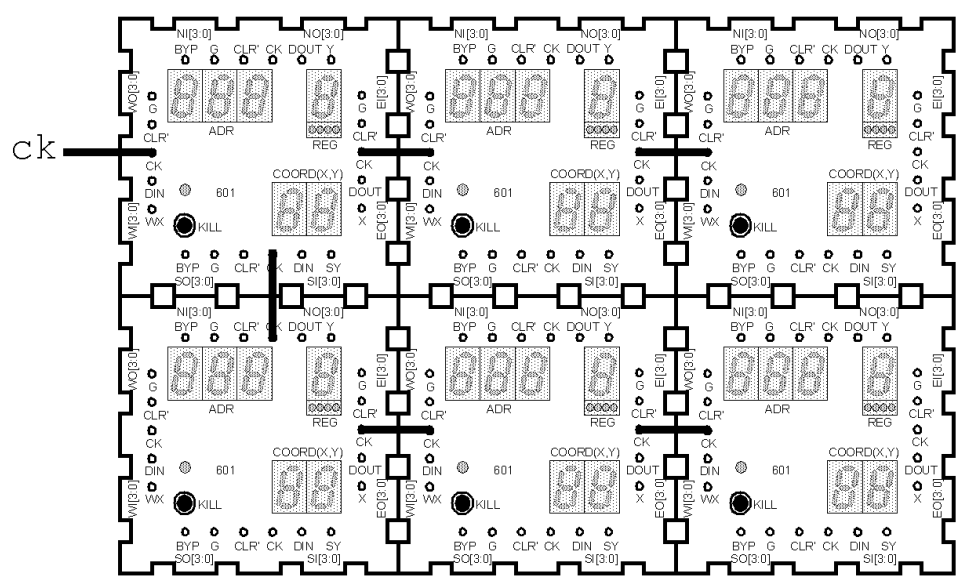

Figure A.4: Exemple de câblage de l'horloge.

Pour des raisons électriques, l'horloge $C K$ tout comme le signal transportant le génome doivent être amplifiés pour attaquer un grand nombre de cellules. Cette amplification peut se faire simplement par adjonction, juste après le module de chargement, d'une porte logique.

\section{A.1.4 Câblage du signal de remise à zéro $(C L R)$}

Le signal de remise à zéro $(C L R)$ indiquant que les biodules doivent se mettre en attente de chargement du génome doit être distribué à tous les biodules (figure A.5). Puisque toutes les $C L R$ d'un biodule sont connectées entre elles, une simple connexion entre chaque cellule voisine permet de diffuser le signal partout.

\section{A.1.5 Câblage pour le transfert du génome (DIN et DOUT)}

Le génome est transmis séquentiellement sur une ligne de donnée. Cette transmission est synchronisée par une horloge $(C K)$. Un câblage par fils entre cellules est nécessaire pour 


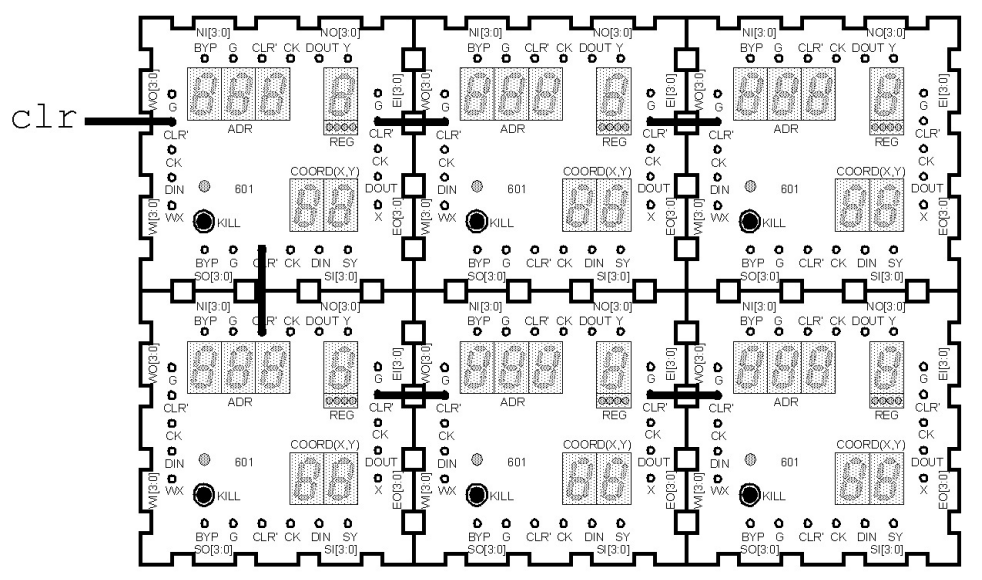

Figure A.5: Exemple de câblage de la remise à zéro.

permettre la transmission du génome de cellule en cellule (figure A.6). Deux entrées DIN et deux sorties DOUT permettent respectivement de faire entrer le génome par le bas ou la gauche de la cellule (South ou West) et de le faire sortir (en direction des voisines) par la droite ou le haut (East ou North).

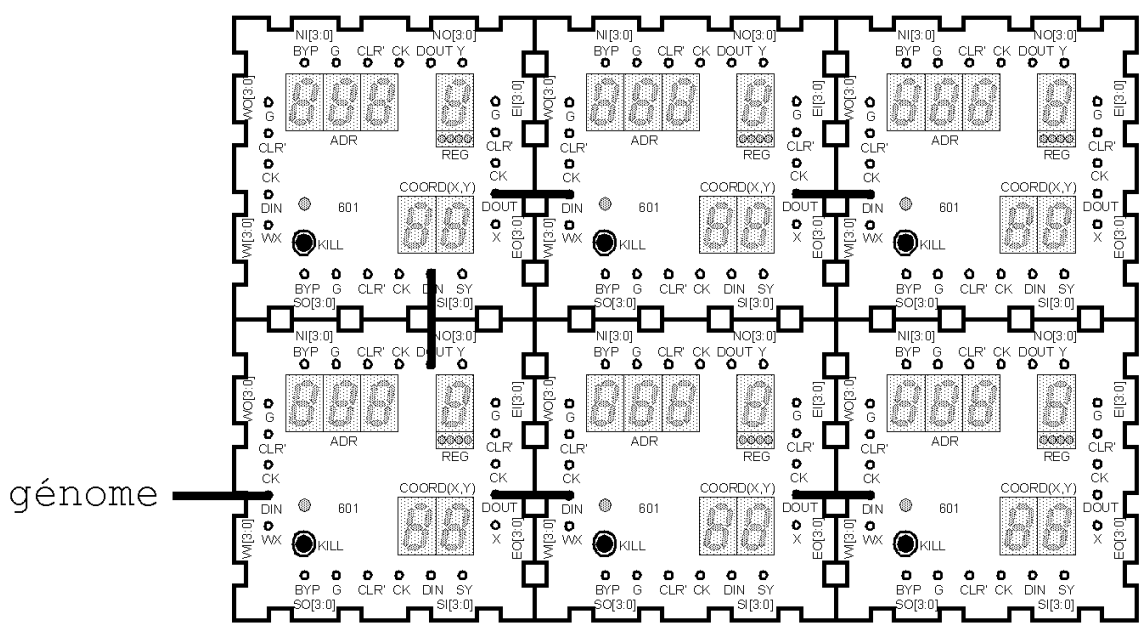

Figure A.6: Exemple de câblage des signaux transportant le génome.

Une seule entrée DIN doit être utilisée. En effet, les deux DIN sont connectés ensemble de manière interne. Il en est de même pour les DOUT.

La synchronisation des données se fait avec le signal d'horloge $C K$. Cette horloge est indispensable pour le chargement et l'exécution et doit aussi être câblée de cellule en cellule. 
Le déclenchement du chargement du génome se fait après remise à zéro de toutes les cellules. Ceci nécessite une courte activation du signal CLR. Les biodules connectés au signal $C L R$ vont s'éteindre et attendre l'arrivée du génome par l'entrée DIN. Après chargement, l'exécution démarre automatiquement.

\section{A.1.6 Câblage des signaux de coordonnées ( $W X, X, S Y$ et $Y)$}

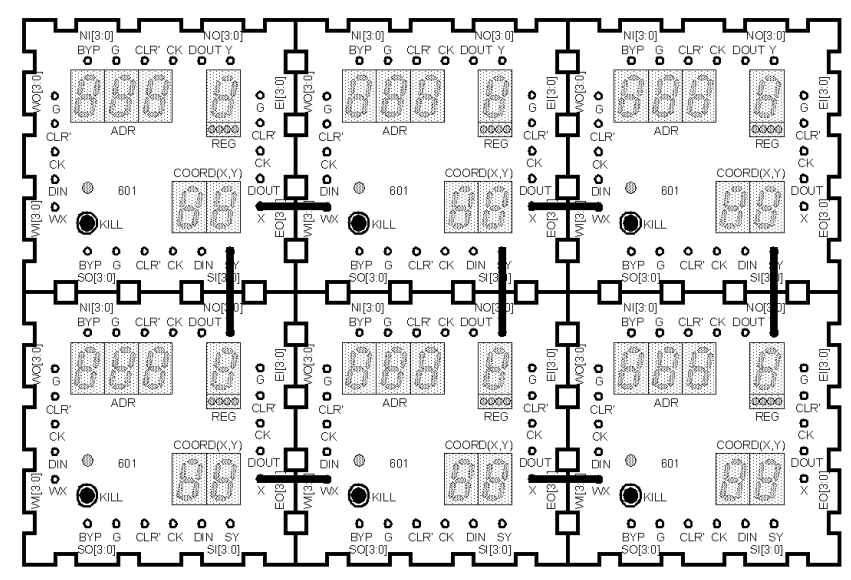

Figure A.7: Exemple de câblage des signaux de position.

Chaque biodule transmet à son voisin de droite (East) sa position en $X$ et à son voisin du haut (North) sa position en $Y$. Pour cette raison, les sorties $X$ et $Y$ se trouvent respectivement à droite (East) et en haut (North) du biodule alors que les entrées $W X$ et $S Y$ se trouvent respectivement à gauche (West) et en bas (South). Alors que les biodules du réseau aux coordonnées $(1, \mathrm{x})$ ou $(\mathrm{x}, 1)$ peuvent rester avec une ou les deux connexions $W X$ et $S Y$ non connectées, les autres doivent être câblés de la manière suivante : $X$ sur $W X$ voisin et $Y$ sur $S Y$ voisin (figure A.7).

\section{A.1.7 Câblage du signal de suicide commun (BYP)}

Le signal $B Y P$ est activé (état logique 1) par une cellule qui est tuée (pression sur $K I L L$, la LED passe du vert au rouge). Ce signal est désactivé par la cellule lorsqu'elle est ressuscitée. Ce signal doit connecter (de $B Y P$ en $B Y P$ ) toutes les cellules susceptibles de mourir ensemble. Le cas le plus classique est la connexion de toutes les cellules d'une même colonne (figure A.8). En effet, la transparence de la cellule se fait horizontalement et non verticalement.

Une ou plusieurs cellules ne peuvent pas activer simultanément $B Y P$. Une activation simultanée de $B Y P$ génère un court-circuit entre les composants pilotant ce signal. Il faut 


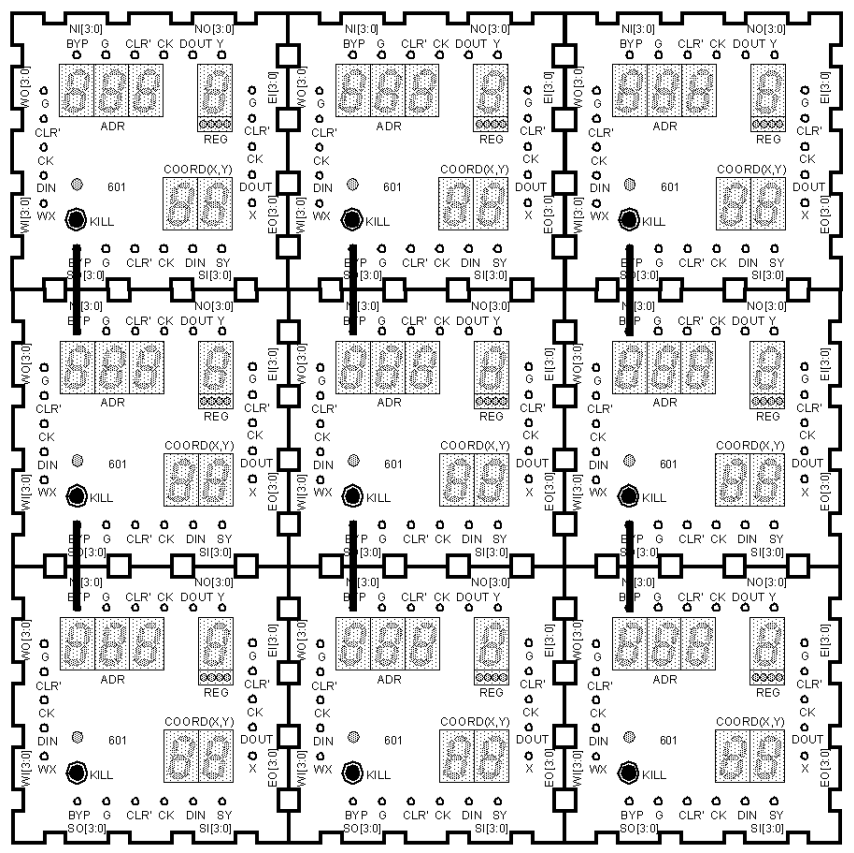

Figure A.8: Exemple de câblage des signaux BYP.

également veiller à ce qu'à la mise sous tension du réseau, plusieurs cellules de la même colonne n'activent pas simultanément le signal $B Y P$.

\section{A.1.8 Câblage du signal de synchronisation global $(G)$}

Le signal de synchronisation $G$ apparaissant quatre fois sur chaque biodule est connecté de manière interne à ces quatre points. Il suffit que chaque cellule soit connectée à une voisine par ce signal et qu'une cellule du réseau soit reliée à un signal donné pour que ce même signal soit distribué dans tout le réseau.

Le signal $G$ peut aussi être vu comme un signal d'entrée supplémentaire indépendamment de toute signification.

\section{A.1.9 Connexion avec le MacIntosh et son interface LSLParallelIO}

La programmation et l'utilisation des biodules 601 se fait à partir du MacIntosh et de son interface LSLParallelIO. L'assembleur asmBio2 et le chargeur macBio2 sont disponibles uniquement pour ce type d'ordinateur.

Cependant la simple utilisation du connecteur venant du Macintosh et du logidule 513 ne suffisent pas; une logique supplémentaire est nécessaire. En effet, il 


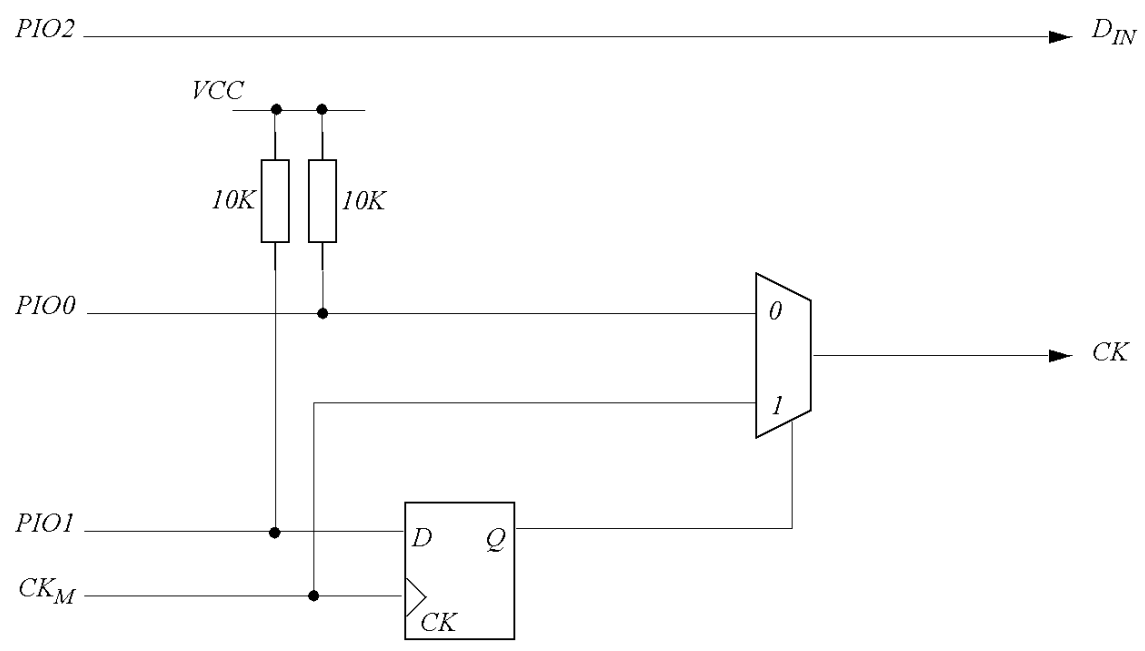

Figure A.9: Connexion des biodules avec l'interface LSLParallelIO du MacIntosh.

est souvent préférable d'utiliser deux horloges différentes pour le biodule : l'une pour charger le génome et l'autre pour faire exécuter le code par le biodule. La première horloge pour le chargement est fournie par la carte parallèle. En revanche, lorsque le chargement est terminé, il peut-être opportun de choisir manuellement la vitesse d'exécution de la machine de décision binaire. Ceci est particulièrement pratique pour exécuter pas à pas un programme.

L'utilisation de deux horloges différentes est facilitée par l'existence d'un signal de sélection SEL provenant de l'interface du MacIntosh et pilotée par le logiciel macBio2. Ce signal permet de choisir quelle horloge doit être distribuée sur les biodules. Le schéma de la figure A.9 permet de garantir une commutation des horloges sans interférence.

Les signaux PIO[2:0] proviennent directement de l'interface LSLParallelIO du MacIntosh. PIO2 transmet les données vers le signal DIN du biodule. PIO1 est le signal de sélection d'horloge. Lorsqu'il est inactif, il indique que l'horloge est fournie par l'interface parallèle. Il s'agit du chargement d'un génome. PIOO est l'horloge fournie par la carte LSLParallelIO. $C K_{M}$ est l'horloge réglée manuellement qui est utilisée pour l'exécution des programmes par la machine de décision binaire. Finalement, $C K$ est le signal destiné au biodule.

\section{A.1.10 Connexion avec le MacIntosh et son interface série LoaderSer}

Un montage similaire à celui utilisé avec LSLParallelIO permet d'employer la sortie série RS-422 du MacIntosh pour charger le génome sur le réseau [44]. Ce montage est constitué d'un logidule (ou socle) pour circuit intégré de type PLCC84 et de deux supports 
pour circuits intégrés DIL (Dual inline) comportant un quartz, un convertisseur mode différentiel-TTL et un circuit programmable de la famile Actel. Le montage complet avec le câble RS-422 est fourni par le laboratoire [44]. Ce montage se connecte sur l'interface série modem du Macintosh par l'intermédiaire du câble RS-422. Les autres connexions sont expliquées ci-dessous.

Sur le montage LOADERSER se trouvent plusieurs signaux importants : une entrée d'horloge, une entrée de remise à zéro, une sortie d'horloge et une sortie de données. L'entrée d'horloge permet à l'utilisateur de fixer la fréquence de fonctionnement des biodules. Le signal de sortie d'horloge qui se connecte aux biodules est équivalent au signal d'entrée d'horloge lorsque le génome est interprété par les biodules. Lors du chargement, le signal de sortie d'horloge est fourni par un quartz ajusté sur la vitesse de transmission de la ligne série du MacIntosh. La transmission du génome se fait par la sortie de données. Finalement, l'entrée de remise à zéro permet de placer le chargeur LOADERSER en mode de réception du génome.

Le chargement du génome du côté du MacIntosh se fait de la même manière qu'avec l'interface LSLParallelIO, mais plutôt que d'utiliser le programme macBio2 il faut employer le programme serBio2.

\section{A.2 Fonctionnement interne}

\section{A.2.1 Caractéristiques générales}

Le biodule 601 se compose des blocs suivants :

1. une machine de décision binaire;

2. une mémoire RAM de 4k x 4 bits (soit 1024 instructions);

3. un routeur de 20 entrées et 16 sorties;

4. un registre de 4 bits à usage général couplé à un affichage 7 segments;

5. deux registres de sortie 4 bits couplés à deux affichages 7; segments qui servent à stocker les valeurs des coordonnées à propager vers les voisins à l'est et au nord dans le réseau.

\section{A.2.2 Machine de décision binaire}

Cette machine exécute des instructions de 16 bits de large. Elle peut accéder à l'ensemble de la mémoire à disposition dans le biodule soit $4 \mathrm{k} \times 4$ bits. Les instructions étant 4 fois plus larges que l'accès à la mémoire, la machine a besoin de 4 cycles d'horloge 
pour rechercher la prochaine instruction à exécuter. Ceci implique évidemment qu'une instruction est exécutée en quatre cycles.

La machine de décision binaire charge son code de manière sérielle par l'entrée DIN du biodule. Un état actif sur l'entrée $C L R$ du biodule provoque la mise en attente du code (génome). Cet état est quitté après que la fin du génome ait été détectée. Cette dernière est signalée par une pseudo-instruction spéciale (SYNC) transmise dans le génome. Lorsque cette instruction est reçue, la machine de décision binaire commence à exécuter le code en partant de l'adresse 0; le chargement du génome est terminé.

\section{A.2.3 Routeur de 20 entrées et 16 sorties}

Le biodule 601 compte 4 bus 4 bits en entrée et 4 bus 4 bits en sortie. Chaque signal de sortie peut être "routé" sur un signal d'entrée ou un bit du registre interne. La modification de ce routage peut être effectué en tout temps. À l'initialisation, le routage n'est pas défini et il convient à l'utilisateur d'imposer ce dont il a besoin. En revanche, lors de la mort d'une cellule, le routage produit une connectique qui rend la cellule transparente. En effet, chaque bus entrant est alors connecté à son correspondant en sortie, disposé sur le côté opposé. Par exemple, le bus EI3:0 sera connecté au bus WO3:0.

\section{A.2.4 Registre 4 bits d'usage général et affichage 7 segments}

Un registre 4 bits d'usage général est accessible par la machine de décision binaire. Il est possible de connecter n'importe quel bit de ce registre à n'importe quel signal de bus en sortie. La valeur du registre est indiquée par un affichage sur la face supérieure du biodule. Sous l'affichage une représentation binaire est également présente.

\section{A.2.5 Registres 4 bits de sortie indiquant la position}

Chaque biodule possède en entrée deux signaux $W X$ et $S Y$ indiquant la position courante du biodule dans le réseau. Ces signaux sont utilisés en conjonction avec le signal d'horloge de la machine de décision binaire pour transmettre en série les positions d'une longueur de 4 bits. Ces signaux sont disponibles en lecture pour le programme s'exécutant dans le biodule. En revanche, les registres qui contiennent les valeurs à transmettre sur $X$ et $Y$ ne sont pas disponibles en lecture.

L'affichage sur la face supérieure du biodule donne la position courante de la cellule. C'est cette valeur qui est transmise par $X$ et $Y$. 


\section{A.2.6 Comportement après suicide de la cellule}

Une fois la cellule tuée, elle se comporte comme un élément transparent : WI3:0 est relié à EO3:0, EI3:0 est relié à WO3:0 et $W X$ est relié à $X$. Elle devient invisible pour ses voisines horizontales. Si la cellule qui est tuée est reliée par le signal $B Y P$ à certaines voisines (typiquement dans la même colonne), celles-ci auront le même comportement que la mourante. De même, si cette dernière est ressuscitée, ces mêmes voisines le seront aussi.

Le signal $B Y P$ est actif lorsque la cellule a été tuée. Il est au repos lorsque la cellule est vivante. Ce signal ne doit pas être activé par plusieurs cellules.

Lorsque la cellule est morte, elle exécute néanmoins son code comme elle le ferait en temps normal. C'est la transparence au niveau des signaux horizontaux qui assure sa passivité.

\section{A.3 Jeu d'instructions et programmation du biodule 601}

Le langage de bas niveau du biodule 601 est constitué par des instructions élémentaires permettant de traduire directement un arbre de décision binaire en une suite d'instructions exécutables. Il n'est cependant pas possible de traduire des sous-programmes car le langage machine ne gère pas l'appel à des sous-programmes. Le langage se résume essentiellement à des affectations de variables, des instructions de routage et une instruction de test avec saut.

Pour le programmeur, 6 types d'instructions différentes apparaissent : une instruction "ne fait rien" NOP, deux instructions de saut (conditionnel ou non) et trois types d'affectation de variables. En fait, les sauts sont toujours conditionnels (une variable constante fausse est disponible), mais le langage propose deux syntaxes différentes.

Il est à noter qu'une pseudo-instruction se réserve un code binaire propre pour indiquer le début et la fin de chargement du génome sur le biodule. Cette instruction SYNC n'est ni décodée ni exécutée par le biodule. De plus, elle n'est pas connue de l'assembleur asmBio2.

\section{A.3.1 Diagramme syntaxique du langage asmBio2}

Un programme asmBio2 est une suite d'instructions, éventuellement préfixées d'une étiquette (un texte suivi de ":"), ou de commentaires. Une instruction peut également être suivie d'un commentaire (voir figure A.10).

Un commentaire est un texte préfixé par le caractère ";". Le commentaire s'arrête lorsque le caractère fin de ligne est détecté (voir figure A.11). 


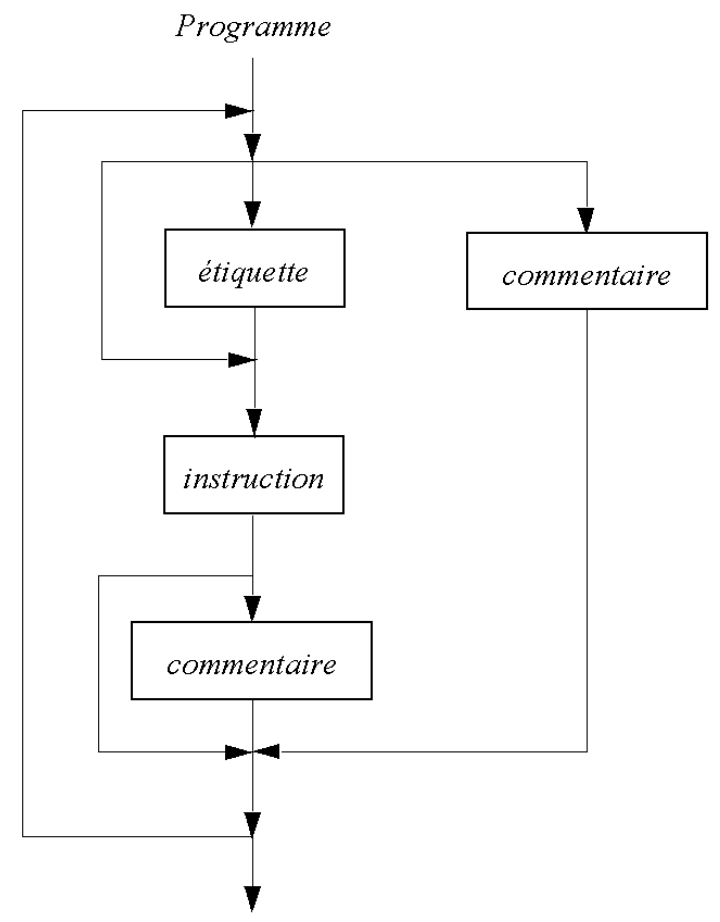

Figure A.10: Structure syntaxique d'un programme asmBio2.

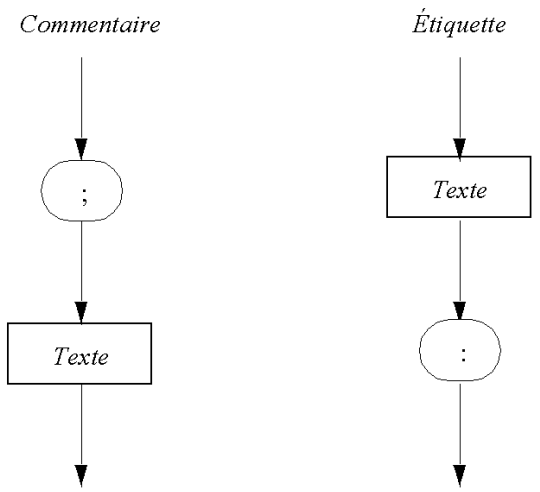

Figure A.11: Structure syntaxique d'un commentaire et d'une étiquette d'un programme asmBio2.

Une instruction commence par l'un des mots clés NOP, GOTO, IF ou DO (voir figure A.12). Le GOTO doit être suivi d'un nom d'étiquette apparaissant quelque part dans le programme. Le IF doit être suivi d'un nom de variable d'entrée suivie du mot clé ELSE et d'un nom d'étiquette apparaissant quelque part dans le programme. Le DO 


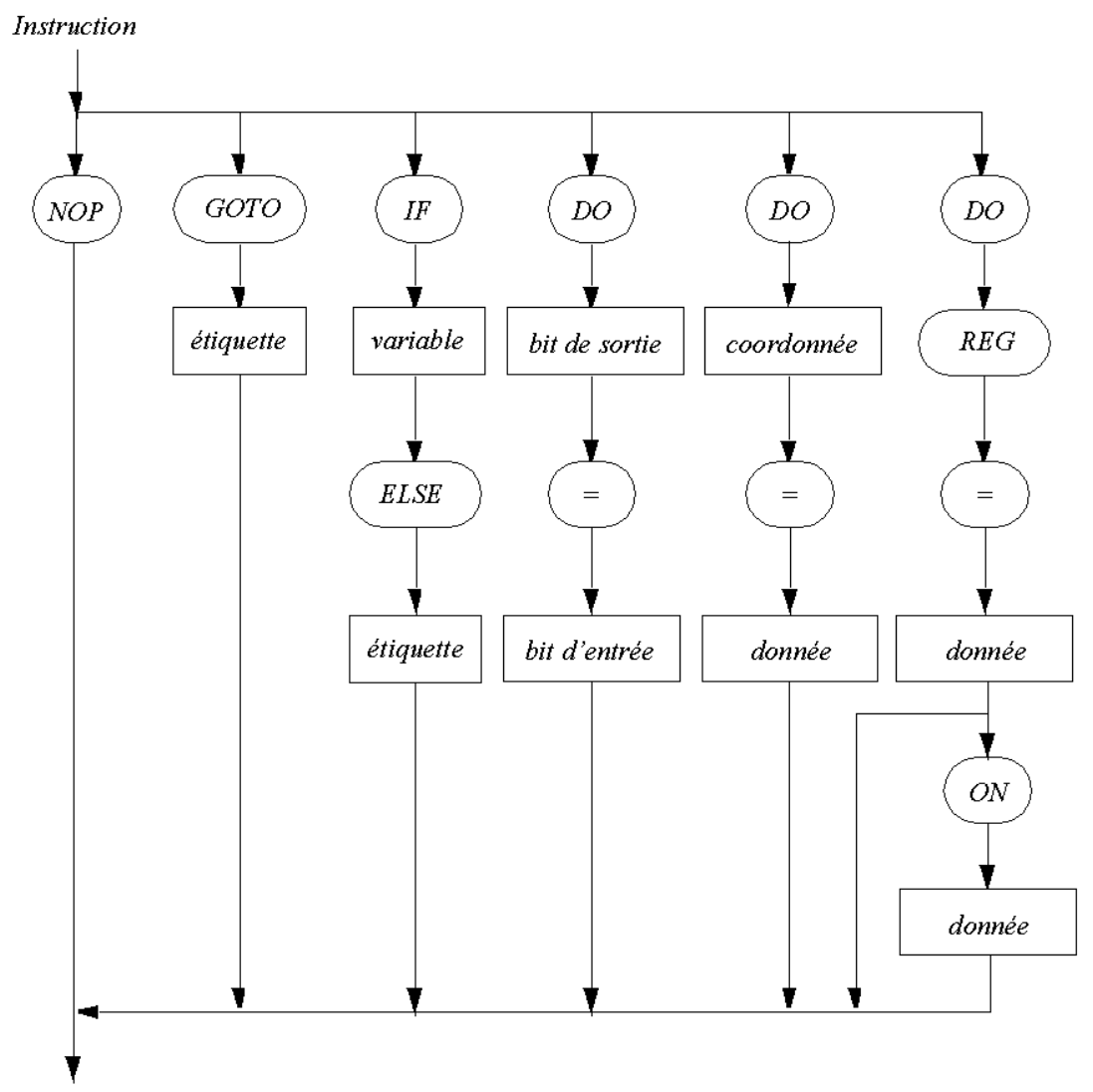

Figure A.12: Structure syntaxique d'une instruction d'un programme asmBio2.

prend trois formes différentes : il peut être suivi du nom d'un signal de sortie, du symbole "=" et d'un nom de signal d'entrée. Il peut être suivi du nom d'une coordonnée (X ou Y), du symbole "=" et d'une valeur décimale comprise entre 0 et 15 . Il peut finalement être suivi par le mot "REG", le symbole "=" et une valeur décimale comprise entre 0 et 15. De plus, cette dernière forme peut être complétée par le mot clé "ON" suivi d'une valeur décimale comprise entre 0 et 15.

\section{A.3.2 Variables}

Une variable est un bit représentant un signal disponible en entrée qui peut être utilisé comme valeur de test. Dans le biodule 601 se trouvent 30 variables. Il y a les 16 signaux des 4 bus (connexions automatiques) en entrée. Il y a les deux bus de 4 bits représentant les coordonnées en $\mathrm{X}$ et en $\mathrm{Y}$ entrantes. Il y a aussi les 4 bits du registre d'usage général. Finalement, il y a le signal de synchronisation général $\mathrm{G}$ ainsi qu'un signal toujours de valeur fausse (utilisé pour réaliser le GOTO).

La liste des variables est : 


$\begin{array}{cccc}N I 3 & \text { NI2 } & \text { NI1 } & \text { NIO } \\ E I 3 & E I 2 & \text { EI1 } & \text { EIO } \\ \text { SI3 } & \text { SI2 } & \text { SI1 } & \text { SIO } \\ W I 3 & W I 2 & W I 1 & W I 0 \\ & & & \\ W X 3 & W X 2 & W X 1 & \text { WXO } \\ \text { SY3 } & \text { SY2 } & \text { SY1 } & \text { SYO } \\ & & & \\ \text { REG3 } & \text { REG2 } & \text { REG1 } & \text { REGO } \\ G & \text { FALSE } & & \end{array}$

\section{A.3.3 Bits de sortie}

Les bits de sortie, ou signaux de sortie, sont ceux que l'utilisateur peut modifier. La modification se fait sur la connexion du signal et non directement sur la valeur transportée par le signal. Un signal de sortie se connecte à un signal d'entrée. Cette connexion peut se faire à n'importe quel moment par le programme à l'aide d'une instruction DO. Il s'agit d'une configuration locale.

À l'initialisation, les connexions sont aléatoires. Après la mort d'une cellule, toutes ses connexions internes entre bits d'entrée et de sortie sont faites de manière à la rendre transparente. Après résurrection de la cellule, la configuration locale précédant la mort est restituée.

La liste des bits de sortie est :

$\begin{array}{llll}\text { NO3 } & \text { NO2 } & \text { NO1 } & \text { NO0 } \\ \text { EO3 } & \text { EO2 } & \text { EO1 } & \text { EO0 } \\ \text { SO3 } & \text { SO2 } & \text { SO1 } & \text { SO0 } \\ \text { WO3 } & \text { WO2 } & \text { WO1 } & \text { WOO }\end{array}$

\section{A.3.4 Bits d'entrée}

Les bits d'entrées, ou signaux d'entrées, sont ceux qui peuvent servir de source à une connexion. En fait, il s'agit d'un sous-ensemble des variables :

$\begin{array}{cccc}\text { NI3 } & \text { NI2 } & \text { NI1 } & \text { NIO } \\ \text { EI3 } & \text { EI2 } & \text { EI1 } & \text { EIO } \\ \text { SI3 } & \text { SI2 } & \text { SI1 } & \text { SIO } \\ \text { WI3 } & \text { WI2 } & \text { WI1 } & \text { WIO } \\ \text { REG3 } & \text { REG2 } & \text { REG1 } & \text { REGO }\end{array}$




\section{A.3.5 Jeu d'instructions}

\section{L'instruction NOP}

\section{Syntaxe : NOP}

L'instruction NOP ne fait rien. Cette instruction peut être utilisée pour garantir un certain délai entre deux paquets d'instructions.

\begin{tabular}{|c|c|c|c|ccc}
15 & 14 & 13 & 12 & 11 & 0 \\
\hline 0 & 0 & 0 & 0 & &
\end{tabular}

Figure A.13: Format de l'instruction NOP.

\section{L'instruction IF}

Syntaxe : IF variable ELSE étiquette

L'instruction de test IF permet de tester une variable et de changer en conséquence l'adresse de la prochaine instruction à exécuter (modification du compteur ordinal PC). Si la variable désignée vaut 1 (test vrai), l'instruction suivante (dans la séquence des instructions) sera exécutée. Sinon, l'instruction suivante est déterminée par l'adresse fournie (saut absolu) dans l'instruction. La figure A.13 presente le format de l'instruction IF et la table A. 2 présente les differents codes des variables.

C'est ce même format d'instruction qui est employé pour réaliser des sauts inconditionnels. Dans ce cas, la variable de test est une constante qui possède la valeur fausse.

\begin{tabular}{|c|ccccc|}
\multicolumn{1}{c|}{15} & 14 & & 10 & 9 & 0 \\
\hline 1 & & Variable & & Adresse \\
\hline
\end{tabular}

Figure A.14: Format des instructions IF et GOTO.

\section{L'instruction GOTO}

Syntaxe : GOTO étiquette

Cette instruction assigne au compteur ordinal la valeur de l'étiquette. 
Table A.2: Codage des variables.

\begin{tabular}{|c|c|c|c|c|c|c|c|}
\hline codes & vare & code & Var & codes & Var & code & $V a r$ \\
\hline 00000 & REGO & 01000 & EIO & 10000 & WIO & 11000 & SYO \\
\hline 00001 & REG1 & 01001 & EI1 & 10001 & WI 1 & 11001 & SY1 \\
\hline 00010 & REG2 & 01010 & EI2 & 10010 & WI2 & 11010 & SY2 \\
\hline 00011 & REG3 & 01011 & EI3 & 10011 & WI 3 & 11011 & SY3 \\
\hline 00100 & NIO & 01100 & SIO & 10100 & WXO & 11100 & G \\
\hline 00101 & NI1 & 01101 & SI1 & 10101 & WX1 & 11101 & FALSE \\
\hline 00110 & NI2 & 01110 & SI2 & 10110 & WX2 & 11110 & FALSE \\
\hline 00111 & NI3 & 01111 & SI3 & 10111 & WX 3 & 11111 & FALSE \\
\hline
\end{tabular}

\section{L'instruction DO (configuration locale)}

Syntaxe : DO signal_de_bus_en_sortie = signal_de_bus_en_entrée

L'instruction DO de configuration locale établit un lien entre un bit de sortie et un bit d'entrée. Le bit de sortie aura donc après connexion, constamment la même valeur que le bit d'entrée.

Il n'est pas possible de connecter une sortie avec une entrée du même côté cardinal. Seules les trois autres points cardinaux différents du bit de sortie ainsi que les bits du registre d'usage général peuvent servir d'entrée.

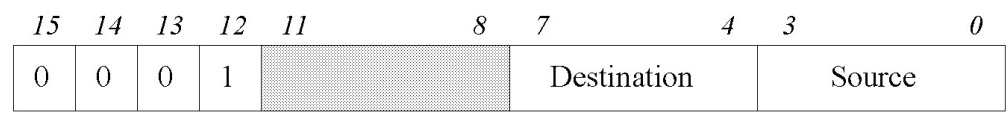

Figure A.15: Format de l'instruction DO (configuration locale).

Table A.3: Codage de la destination.

\begin{tabular}{|c|c|c|c|c|c|c|c|}
\hline Dest & Var & Dest & Var & Dest & Var & Dest & Var \\
\hline 0000 & NOO & 0100 & EOO & 1000 & SOO & 1100 & WOO \\
\hline 0001 & NO1 & 0101 & E01 & 1001 & SO1 & 1101 & WO1 \\
\hline 0010 & NO2 & 0110 & EO2 & 1010 & $\mathrm{SO} 2$ & 1110 & WO2 \\
\hline 0011 & NO3 & 0111 & EO3 & 1011 & $\mathrm{SO} 3$ & 1111 & WO 3 \\
\hline
\end{tabular}


Table A.4: Codage de la source si la destination=00xx.

\begin{tabular}{|l|l|l|l|c|c|c|c|c|}
\hline Src & Var & Src & Var & Src & Var & Src & Var \\
\hline 0000 & REGO & 0100 & EIO & 1000 & SI0 & 1100 & WIO \\
\hline 0001 & REG1 & 0101 & EI1 & 1001 & SI1 & 1101 & WI1 \\
\hline 0010 & REG2 & 0110 & EI2 & 1010 & SI2 & 1110 & WI2 \\
\hline 0011 & REG3 & 0111 & EI3 & 1011 & SI3 & 1111 & WI3 \\
\hline
\end{tabular}

Table A.5: Codage de la source si la destination $=01 \mathrm{xx}$.

\begin{tabular}{|c|c|c|c|c|c|c|c|}
\hline Srce & Var & Src & Var & Srac & Var & Sre & Var \\
\hline 0000 & REGO & 0100 & NIO & 1000 & SIO & 1100 & WIO \\
\hline 0001 & REG1 & 0101 & NI1 & 1001 & SI1 & 1101 & WI1 \\
\hline 0010 & REG2 & 0110 & NI2 & 1010 & SI2 & 1110 & WI 2 \\
\hline 0011 & REG3 & 0111 & NI 3 & 1011 & SI 3 & 1111 & WI 3 \\
\hline
\end{tabular}

Table A.6: Codage de la source si la destination=10xx.

\begin{tabular}{|c|c|c|c|c|c|c|c|}
\hline Sro & Var & Sro & Var & Sro & Var & Srac & Var \\
\hline 0000 & REGO & 0100 & NIO & 1000 & EIO & 1100 & WIO \\
\hline 0001 & REG1 & 0101 & NI1 & 1001 & EI1 & 1101 & WII \\
\hline 0010 & REG2 & 0110 & NI2 & 1010 & EI2 & 1110 & WI2 \\
\hline 0011 & REG3 & 0111 & NI3 & 1011 & EI3 & 1111 & WI 3 \\
\hline
\end{tabular}

Table A.7: Codage de la source si la destination $=11 \mathrm{xx}$.

\begin{tabular}{|c|c|c|c|c|c|c|c|}
\hline Src & var & Src & var & SrC & var & Src & var \\
\hline 0000 & REGO & 0100 & NIO & 1000 & EIO & 1100 & SIO \\
\hline 0001 & REGI & 0101 & NII & 1001 & EI1 & 1101 & SI1 \\
\hline 0010 & REG2 & 0110 & NI2 & 1010 & EI2 & 1110 & SI2 \\
\hline 0011 & REG3 & 0111 & NI 3 & 1011 & EI3 & 1111 & SI3 \\
\hline
\end{tabular}

\section{L'instruction DO (position)}

Syntaxe : DO coordonnée $=$ valeur4

Cette instruction place la valeur de 4 bits dans le registre de coordonnée spécifié (coordonnée $=X$ ou $Y$ ). Cette valeur est ensuite exportée (signaux $X$ et $Y$ ) vers la cellule voisine et affichée sur l'affichage sept segments correspondant à la coordonnée ( $X$ ou $Y)$ 
placé en bas à droite sur la face supérieure du biodule.

\begin{tabular}{|l|l|l|l|l|l|l|}
15 & 14 & 13 & 12 & 11 & 4 & 3 \\
\hline 0 & Coord & 1 & & Valeur $_{4}$ \\
\hline
\end{tabular}

Figure A.16: Format de l'instruction DO (position).

Table A.8: Codage de la coordonnée.

\begin{tabular}{|c|c|}
\hline Coord & Code \\
\hline$X$ & 01 \\
\hline$Y$ & 10 \\
\hline
\end{tabular}

\section{L'instruction DO (registre)}

Syntaxe : DO REG = valeur 4

DO $\mathrm{REG}=$ valeur4 ON masque 4

Cette instruction affecte une valeur 4 bits au registre d'usage général. Cette valeur peut être appliquée en utilisant un masque indiquant quels bits sont à mettre à jour dans le registre (tous ceux ou masque vaut 1). Lorsque le "ON masque" est omis, tous les bits du registre sont mis à jour suivant valeur4. Cela revient à écrire DO REG $=\ldots$ ON F.

\begin{tabular}{|c|c|c|c|c|c|c|c|c|}
\hline 15 & 14 & 13 & 12 & 11 & 8 & 7 & 4 & 3 \\
\hline 0 & 1 & 1 & 1 & & & Masque $_{4}$ & & Valeur $_{4}$ \\
\hline
\end{tabular}

Figure A.17: Format de l'instruction DO (registre).

\section{L'instruction SYNC}

\section{Syntaxe : SYNC}

Cette instruction n'est pas disponible à l'utilisateur. Elle sert uniquement lors du chargement du génome. Cependant, dans un soucis de complétude, son format est donné ici.

\begin{tabular}{|c|c|c|c|c|c|c|}
15 & 14 & 13 & 12 & 11 & 0 \\
0 & 1 & 1 & 0 & & 0
\end{tabular}

Figure A.18: Format de l'instruction SYNC. 



\title{
Appendix B
}

\section{Complete universal Turing machine microprogram}

\author{
$1 \mathrm{REG}=0$ \\ 24 do $W 03=R E G 3$ \\ $2 \mathrm{X}=0$ \\ 25 do $\mathrm{WO} 2=\mathrm{REG} 2$ \\ $3 \mathrm{Y}=0$ \\ 26 do $W 01=R E G 1$ \\ 27 do $W O 0=R E G O$ \\ 4 Program: \\ 5 if $G$ else TestG \\ 28 Config21: \\ 29 do $\mathrm{EO} 3=\mathrm{SI} 3$ \\ 6 XYInitlocalconfig: \\ 30 do $\mathrm{EO} 2=\mathrm{SI} 2$ \\ 7 if $w X 3$ else $w 81$ \\ 8 do $X=9$ \\ 9 if SY1 else R 45 \\ 10 do $Y=0$ \\ 11 do $R E G=0$ \\ 31 do $\mathrm{EO}=\mathrm{SI} 1$ \\ 32 do $\mathrm{EOO}=\mathrm{SIO}$ \\ 33 goto Program \\ 34 R5: \\ 35 do $\mathrm{Y}=1$ \\ 36 do $R E G=5$ \\ 13 do $\mathrm{SO} 3=\mathrm{NI} 3$ \\ 14 goto Program \\ 37 Config12: \\ 38 do $\mathrm{E} 01=\mathrm{WI} 1$ \\ 15 R45: \\ 16 if SYO else R5 \\ 39 Config1211: \\ 17 do $\mathrm{Y}=2$ \\ 40 do $N O O=R E G O$ \\ 18 do $R E G=4$ \\ 41 do $N O 1=R E G 1$ \\ 42 do $\mathrm{NO} 2=\mathrm{REG} 2$ \\ 19 Config2: \\ 43 do $N O 3=R E G 3$ \\ 20 do $\mathrm{SO} 3=\mathrm{WI} 3$ \\ 21 do $\mathrm{SO} 2=\mathrm{WI} 2$ \\ 44 do $E O O=S I O$ \\ 22 do $S 01=W I 1$ \\ 45 do $\mathrm{SOO}=\mathrm{WIO}$ \\ 23 do $\mathrm{SOO}=\mathrm{WIO}$ \\ 46 do $\mathrm{S} 01=\mathrm{WI} 1$ \\ 47 do $\mathrm{EO}=\mathrm{WI} 3$
}


48 do $\mathrm{SO} 3=\mathrm{WI} 3$

49 goto Program

50 W81:

51 if $W X 2$ else $W 41$

52 if $W X 1$ else $W 65$

53 if $W X 0$ else $X 7$

54 do $X=8$

55 if $S Y 1$ else $R 6 B$

56 do $Y=0$ do $R E G=0$

57 do $W O O=R E G 3$

58 Config2A8:

59 do $\mathrm{EOO}=\mathrm{WIO}$

60 do $\mathrm{EO} 1=\mathrm{WI} 1$

61 do $\mathrm{EO} 2=\mathrm{WI} 2$

62 do $\mathrm{EO} 3=\mathrm{WI} 3$

63 Config1A8:

64 do $\mathrm{NOO}=\mathrm{REGO}$

65 goto Initial

66 R6B :

67 if $S Y O$ else $R B$

68 do $Y=2$

69 do $R E G=6$

70 goto Config2

$71 \mathrm{RB}$ :

72 do $\mathrm{Y}=1$

73 do $R E G=11$

74 goto Config12

$75 \times 7$ :

76 do $X=7$

77 if $S Y 1$ else $R 89$

78 do $Y=0$

79 do $R E G=1$

80 Config2A7:

81 do $W O O=R E G O$

82 goto Config2A8

83 R89:

84 if SYO else $R 9$

85 do $Y=2$

86 do $\mathrm{REG}=8$

87 goto Config2
88 R9:

89 do $Y=1$

90 do $\mathrm{REG}=9$

91 goto Config12

92 W65:

93 if $\mathrm{WX} 0$ else $\mathrm{X} 5$

94 do $X=6$

95 if $S Y 1$ else $R B 4$

96 do $Y=0$

97 do $\mathrm{REG}=0$

98 do $\mathrm{WO} 0=\mathrm{EIO}$

99 goto Config2A8

100 RB4:

101 if SYO else $R 4$

102 do $Y=2$

103 do $\mathrm{REG}=11$

104 goto Config2

$105 \mathrm{R} 4$ :

106 do $Y=1$

107 do $\mathrm{REG}=4$

108 goto Config12

$109 \times 5$ :

110 do $X=5$

111 if $S Y 1$ else $R 4 A$

112 do $Y=0$

113 do $R E G=1$

114 goto Config2A7

115 R4A:

116 if $S Y O$ else $R A$

117 do $\mathrm{Y}=2$

118 do $\mathrm{REG}=4$

119 goto Config2

$120 \mathrm{RA}$ :

121 do $\mathrm{Y}=1$

122 do $\mathrm{REG}=10$

123 goto Config12

124 W41:

125 if $W X 1$ else $W 21$

126 if $\mathrm{WX} 0$ else $\mathrm{X} 3$

127 do $X=4$ 
128 if $\mathrm{SY} 1$ else $\mathrm{R} 98$

129 do $\mathrm{Y}=0$

130 do $\mathrm{REG}=0$

131 goto Config2A7

132 R98:

133 if SYO else R8

134 do $Y=2$

135 do $\mathrm{REG}=9$

136 goto Config2

137 R8 :

138 do $Y=1$

139 do $\mathrm{REG}=8$

140 Config11:

141 do $\mathrm{EO}=\mathrm{WIO}$

142 goto Config1211

$143 \times 3$ :

144 do $X=3$

145 if $S Y 1$ else RA6

146 do $Y=0$

147 do $\mathrm{REG}=1$

148 do $\mathrm{WO} 1=\mathrm{EIO}$

149 goto Config2A7

150 RA6:

151 if SYO else $R 6$

152 do $Y=2$

153 do $R E G=10$

154 goto Config2

155 R6:

156 do $Y=1$

157 do $\mathrm{REG}=6$

158 goto Config11

159 W21:

160 if $W X 0$ else $X 1$

161 do $X=2$

162 if $S Y 1$ else $R 56$

163 do $Y=0$

164 do $R E G=0$

165 goto Config2A7

166 R56:

167 if SYO else $R 6$

168 do $Y=2$
169 do $\mathrm{REG}=5$

170 goto Config2

$171 \mathrm{X} 1$ :

172 do $X=1$

173 if $S Y 1$ else $R 52$

174 do $\mathrm{Y}=0$

175 do $\mathrm{REG}=0$

176 do $\mathrm{EOO}=\mathrm{SIO}$

177 do $\mathrm{EO} 1=\mathrm{SI} 1$

178 do $\mathrm{EO2}=\mathrm{SI} 2$

179 do $\mathrm{EO}=\mathrm{SI} 3$

180 goto ConfiglA8

181 R52:

182 if SYO else R2

183 do $Y=2$

184 do $\mathrm{REG}=5$

185 Config3:

186 do $\mathrm{SO} 3=\mathrm{REG} 3$

187 do $\mathrm{SO} 2=\mathrm{REG} 2$

188 do $\mathrm{SO} 1=\mathrm{REG} 1$

189 do $\mathrm{SOO}=\mathrm{REGO}$

190 do $\mathrm{NO3}=\mathrm{REG} 3$

191 do $\mathrm{NO} 2=\mathrm{REG} 2$

192 do $\mathrm{NO}=\mathrm{REG1}$

193 do $N O O=R E G O$

194 goto Config21

195 R2:

196 do $\mathrm{Y}=1$

197 do $\mathrm{REG}=2$

198 goto Configl1

199 TestG:

200 if $\mathrm{G}$ else Opgenome

201 goto TestG

202 Opgenome:

203 if SY1 else Y21

204 if $W X 2$ else RegQSt3

205 if $W X 1$ else RegQLS

206 if WXO else RegQC

207 RegQR:

208 if NI1 else ToNop 
209 if WI3 else ToNop

210 if WII else QRSRSL

211 goto ToNop

212 QRSRSL:

213 if WIO else QRSL

214 QRSR:

215 if $R E G 3$ else $Q R S R B O$

216 if $R E G 2$ else $Q R S R D 4$

217 if REG1 else QRSRE6

218 if NIO else TO7

219 goto $\mathrm{TOF}$

220 QRSRE6:

221 if NIO else To6

222 goto TOE

223 QRSRD4:

224 if $R E G 1$ else $Q R S R C 4$

225 if NIO else To5

226 goto TOD

227 QRSRC4 :

228 if NIO else To4

229 goto ToC

230 QRSRB 0:

231 if $R E G 2$ else QRSR90

232 if REG1 else QRSRA2

233 if NIO else $\mathrm{TO} 3$

234 goto $T O B$

235 QRSRA2:

236 if NIO else To2

237 goto TOA

238 QRSR90:

239 if $R E G 1$ else $Q R S R 80$

240 if NIO else To1

241 goto To9

242 QRSR80:

243 if NIO else ToO

244 goto To8

245 QRSL:

246 if $R E G 2$ else QRSL60
247 if REG1 else QRSLA8

248 if REGO else TOC

249 goto $T O E$

250 QRSLA8:

251 if REGO else TO8

252 goto $T O A$

253 QRSL60:

254 if REG1 else QRSL20

255 if REGO else TO4

256 goto To6

257 QRSL20:

258 if REGO else TOO

259 goto To2

260 RegQC:

261 if NI1 else ToNop

262 if WI3 else ToNop

263 if WI1 else Sh if $t$

264 if WIO else TOO

265 goto To1

266 Sh ift :

267 if WIO else Sh iftLeft

268 if NIO else TOO

269 goto To1

270 ShitLeft:

271 if EIO else TOO

272 goto To1

273 RegQLS:

274 if WXo else RegS

275 RegQL:

276 if NI1 else ToNop

277 if WI3 else TONop

278 if WI1 else QLSRSL

279 goto ToNop

280 QLSRSL:

281 if WIO else QLSL

282 QLSR:

283 if REG3 else QLSR30

284 if REG2 else QLSR54 
285 if $R E G 1$ else To6

286 To7:

287 if $\mathrm{G}$ else To7

288 do $\mathrm{REG}=7$

289 goto TestG

290 QLSR54:

291 if REG1 else To4

292 goto To5

293 QLSR30:

294 if REG2 else QLSR10

295 if REG1 else To2

296 To3:

297 if $\mathrm{G}$ else To3

298 do $R E G=3$

299 goto TestG

300 QLSR10:

301 if REG1 else To0

302 goto To1

303 QLSL:

304 if REG2 else QLSL70

305 if REG1 else QLSLB8

306 if REGO else QLSLDC

307 if EIO else TOE

308 TOF:

309 if $\mathrm{G}$ else $\mathrm{TOF}$

310 do $R E G=15$

311 goto TestG

312 TOE:

313 if $G$ else $T O E$

314 do $R E G=14$

315 goto TestG

316 QLSLDC:

317 if $\mathrm{EIO}$ else TOC

318 TOD:

319 if $\mathrm{G}$ else $\mathrm{TOD}$

320 do $\mathrm{REG}=13$

321 goto TestG
322 TOC:

323 if $\mathrm{G}$ else $\mathrm{TOC}$

324 do $R E G=12$

325 goto TestG

326 QLSLB8 :

327 if REGO else QLSL98

328 if EIO else TOA

329 goto $\mathrm{TOB}$

330 QLSL98:

331 if EIO else To8

332 goto To9

333 QLSL70:

334 if REG1 else QLSL30

335 if REGO else QLSL54

336 if EIO else To6

337 goto To7

338 QLSL54:

339 if EIO else TO4

340 goto To5

341 QLSL30:

342 if REGO else QLSL10

343 if EIO else TO2

344 goto To3

345 QLSL10:

346 if EIO else ToO

347 goto To1

348 Regs:

349 if $\mathrm{NI1}$ else TONOP

350 if WI3 else To00

351 if NIO else ToO

352 goto To1

353 To00:

354 if WIO else TONOpO

355 goto To0

356 ToNop0 :

357 if WI2 else LoadQC

358 goto ToNop

359 LoadQC: 
360 if EIO else ToO

361 goto $\mathrm{To} 1$

362 RegQSt3:

363 if $W X 1$ else $S t 2 S t 3$

364 if $W X 0$ else $S t 1$

365 RegQ:

366 if NIO else ToNop

367 if WI3 else To1Nop

368 if WIO else ToO

369 goto To1

370 To1Nop:

371 if WIO else ToNop

372 if EIO else TOO

373 goto To1

374 St1:

375 if WI2 else ToNop

376 if WIO else TolExec1

377 if EIO else TOO

378 Exec1:

379 if REGO else TOO

380 To1:

381 if $\mathrm{G}$ else $\mathrm{To} 1$

382 do $R E G=1$

383 goto TestG

384 To0:

385 if $G$ else To0

386 do $\mathrm{REG}=0$

387 goto TestG

388 To1Exec1:

389 if WII else To1To0

390 if NIO else Exec1

391 goto $\mathrm{To1}$

392 TolTo0:

393 if NIO else TOO

394 goto To1

395 ToNop:

396 if $G$ else ToNop

397 goto TestG
398 St2St3:

399 if WXO else st3

400 St2:

401 if WI2 else ToNop

402 if WIO else TolExec2

403 if EI1 else Exec2

404 goto To0

405 Exec2:

406 if EIO else TOO

407 goto $\mathrm{To1}$

408 To1Exec2:

409 if WI1 else $\mathrm{Exec} 2$

410 if NIO else TOO

411 goto To1

412 St3:

413 if SI2 else ToNop

414 if SIO else TOONOP

415 if EIO else TOO

416 goto $\mathrm{TO}$

417 To0Nop:

418 if SI1 else ToNop

419 goto To0

420 Y21:

421 if SYO else TestNI

422 if $W X 3$ else TestEI

423 TestSI:

424 if $\mathrm{SI} 3$ else $\mathrm{SI52}$

425 if SI1 else SI98

426 if SIO else TOA

427 ToB :

428 if $\mathrm{G}$ else $\mathrm{TOB}$

429 do $R E G=11$

430 goto TestG

431 TOA:

432 if $G$ else $T O A$

433 do $\mathrm{REG}=10$

434 goto TestG 



\section{Bibliography}

[1] M. Abramovici and C. Stroud. No-overhead BIST for FPGAs. In Proceedings of First IEEE International On-Line Testing Workshop, pages 90-92, Nice, France, 1995.

[2] M. A. Arbib. Theories of Abstract Automata. Prentice-Hall, Englewood Cliffs, N.J., 1969.

[3] M. A. Arbib. Handbook of Brain Theory and Neural Networks. MIT Press, Cambridge, MA, 1995.

[4] W. Asprey. John von Neumann and the Origins of Modern Computing. MIT Press, Cambridge, MA, 1992.

[5] E. R. Banks. Universality in Cellular Automata. In IEEE 11th Annual Symposium on Switching and Automata Theory, pages 194-215, Santa Monica, CA, October 1970 .

[6] J. Barwise and J. Etchemendy. Turing's World 3.0. Center for the Study of Language and Information, Stanford, CA, 1993.

[7] C. H. Bennett. Notes on the history of reversible computation. IBM Journal of Research and Development, 32(1):16-23, 1988.

[8] C. H. Bennett and R. Landauer. The fundamental limits of computation. Scientific American, pages 48-56, July 1985.

[9] J.-L. Beuchat and J.-O. Haenni. Von Neumann's 29-State Cellular Automaton: A Hardware Implementation. IEEE Transactions on Education, 43(3):300-308, August 2000.

[10] F. Bois. Un simulateur JAVA pour réseaux de cellules MICTREE. Technical report, École Polytechnique Fédérale de Lausanne, Laboratoire de systèmes logiques, Lausanne, Switzerland, June 2001.

[11] J. Bowles. A Survey of Reliability-Prediction Procedures For Microelectronic Devices. IEEE Trans. on Reliability, 41(1):2-12, March 1992.

[12] S.D. Brown, R.J. Francis, J. Rose, and Z.G. Vranesic. Field-Programmable Gate Arrays. Kluwer Academic Publishers, Boston, 1992. 
[13] R. E. Bryant. Symbolic boolean manipulation with ordered binary-decision diagrams. ACM Computing Surveys, 24(3):293-318, 1992.

[14] A. Burks, editor. Essays on Cellular Automata. University of Illinois Press, Urbana, Illinois, 1970.

[15] J. Byl. Self-Reproduction in Small Cellular Automata. Physica D, 34:295-299, 1989.

[16] E. F. Codd. Cellular Automata. Academic Press, New York, 1968.

[17] C. Darwin. The Origin of Species by Means of Natural Selection. John Murray, London, 1859.

[18] M. Davio, J.-P. Deschamps, and A. Thayse. Digital Systems with Algorithm Implementation. John Wiley, Chichester, 1983.

[19] S. Durand, A. Stauffer, and D. Mange. Biodule: An Introduction to Digital Biology. Technical report, École Polytechnique Fédérale de Lausanne, Laboratoire de systèmes logiques, Lausanne, Switzerland, September 1994.

[20] J-P. Erard and P. Déguénon. Simulation par événements discrets. Presses polytechniques et universitaires romandes, Lausanne, Switzerland, 1996.

[21] D. B. Fogel. Evolutionary Computation: Toward a New Philosophy of Machine Intelligence. IEEE Press, Piscataway, NJ, 1995.

[22] S. Forrest, P. Burrows, and M. Thompson. The dawn of organic electronics. IEEE Spectrum, 37(8):29-34, August 2000.

[23] M. Goeke. Documentation technique du Biodule 2. Technical report, École Polytechnique Fédérale de Lausanne, Laboratoire de systèmes logiques, Lausanne, Switzerland, May 1995.

[24] M. Goeke, M. Sipper, D. Mange, A. Stauffer, E. Sanchez, and M. Tomassini. Online Autonomous Evolware. In T. Higuchi, M. Iwata, and L. Weixin, editors, Evolvable Systems: From Biology to Hardware, volume 1259 of Lecture Notes in Computer Science, pages 96-106. Springer-Verlag, Berlin, 1996.

[25] M. H. Hassoun. Fundamentals of Artificial Neural Networks. MIT Press, Cambridge, MA, 1995.

[26] G. T. Herman. On Universal Computer-Constructors. Information Processing Letters, 2(3):61-64, August 1973.

[27] P. Hertzog. Simulateur pour réseaux de cellules MICTREE. Technical report, École Polytechnique Fédérale de Lausanne, Lausanne, Switzerland, February 2000.

[28] A. Hodges. Alan Turing and the Turing Machine. In R. Herken, editor, The Universal Turing Machine a Half Century Survey, pages 3-14. Springer-Verlag, Wien, second edition, 1995. 
[29] J. H. Holland. Hidden Order: How Adaptation Builds Complexity. Addison Wesley, New York, 1995.

[30] J. E. Hopcroft and J. D. Ullman. Introduction to Automata Theory Languages and Compilation. Addison-Wesley, Redwood City, CA, 1979.

[31] P. D. Hortensius, R. D. McLeod, and B. W. Podaima. Cellular automata circuits for built-in self-test. IBM Journal of Research and Development, 34(2/3):389-405, 1990.

[32] D. C. Ince, editor. Mechanical Intelligence: Collected Works of A. M. Turing, chapter Intelligent Machinery, pages 107-128. North-Holland, Amsterdam, 1992.

[33] K. Kattel, S. Zhang, M. Serra, and J. C. Muzio. 2-by-n Hybrid Cellular Automata with Regular Configuration: Theory and Application. IEEE Transactions on Computers, 48(3):285-295, March 1999.

[34] S. C. Kleene. Turing's Analysis of Computability, and Major Applications of It. In R. Herken, editor, The Universal Turing Machine a Half Century Survey, pages 15-49. Springer-Verlag, Wien, second edition, 1995.

[35] J. R. Koza. Spontaneous emergence of self-replicating and evolutionarily selfimproving computer programs. In Christopher G. Langton, editor, Artificial Life III, volume XVII of SFI Studies in the Sciences of Complexity, pages 225-262. Addison-Wesley, Redwood City, CA, USA, 1994.

[36] J.R. Koza. Genetic Programming. The MIT Press, Cambridge, MA, 1992.

[37] J.R. Koza. Genetic Programming II. The MIT Press, Cambridge, MA, 1994.

[38] C. G. Langton. Self-reproduction in cellular automata. Physica D, 10:135-144, 1984.

[39] C. Lee. Synthesis of a Cellular Computer. In J. J. Tou, editor, Applied Automata Theory, pages 217-234. Academic Press, London, 1968.

[40] C. Y. Lee. Automata and Finite Automata. Bell System Tech. Journal, XXXIX:1267-95, 1960.

[41] Steven Levy. Artificial Life: The Quest For A New Creation. Pantheon Books, New York, 1992.

[42] H.R. Lewis and C.H. Papadimitriou. Elements of the Theory of Computation. Prentice-Hall, Englewood Cliffs, NJ, 1981.

[43] A. Lindermayer. Mathematical models for cellular interaction in development. parts i and ii. Journal of Theoretical Biology, 18:280-315, 1968.

[44] D. Madon. LoaderSer: Description et utilisation. Technical report, École Polytechnique Fédérale de Lausanne, Laboratoire de systèmes logiques, Lausanne, Switzerland, March 1996. 
[45] D. Madon. Architecture massivement parallèle tolérant des défaillances. In RenPar'9, 9e rencontres francophones du parallélisme, pages 189-192, Lausanne, Switzerland, May 1997.

[46] D. Madon. Biodule 601: Description et utilisation. Technical report, École Polytechnique Fédérale de Lausanne, Laboratoire de systèmes logiques, Lausanne, Switzerland, 1998.

[47] D. Mange. Microprogrammed Systems: An Introduction to Firmware Theory. Chapman \& Hall, London, 1992. (First published in French as "Systèmes microprogrammés: une introduction au magiciel”, Presses Polytechniques et Universitaires Romandes, Lausanne, Switzerland, 1990).

[48] D. Mange. Teaching firmware as a bridge between hardware and software. IEEE Transactions on Education, 36(1):152-157, 1993.

[49] D. Mange, M. Goeke, D. Madon, A. Stauffer, G. Tempesti, and S. Duran. Embryonics: A New Family of Coarse-grained Field-Programmable Gate Array with Self-Repair and Self-Reproducing Properties. In E. Sanchez and M. Tomassini, editors, Towards Evolvable Hardware, pages 197-220. Springer-Verlag, Berlin, 1996.

[50] D. Mange, D. Madon, A. Stauffer, and G. Tempesti. Von Neumann revisited: A Turing machine with self-repair and self-reproduction properties. Robotics and Autonomous Systems, 22(1):35-58, 1997.

[51] D. Mange, E. Sanchez, A. Stauffer, G. Tempesti, P. Marchal, and C. Piguet. Embryonics: A New Methodology for Designing Field-Programmable Gate Arrays with Self-Repair and Self-Replicating Properties. IEEE Transactions on VLSI Systems, 6(3):387-399, September 1998.

[52] D. Mange, M. Sipper, A. Stauffer, and G. Tempesti. Towards Robust Integrated Circuits: The Embryonics Approach. Proceedings of the IEEE, 88(04):516-541, April 2000.

[53] D. Mange, A. Stauffer, and G. Tempesti. Binary Decision Machine-Based Cells. In D. Mange and M. Tomassini, editors, Bio-Inspired Computing Machines: Toward Novel Computational Architectures, pages 183-216. Presses Polytechniques et Universitaires Romandes, Lausanne, Switzerland, 1998.

[54] D. Mange, A. Stauffer, and G. Tempesti. Embryonics: A Macroscopic View of the Cellular Architecture. In M. Sipper, D. Mange, and A. Pérez-Uribe, editors, Evolvable Systems: From Biology to Hardware, volume 1478 of Lecture Notes in Computer Science, pages 174-184. Springer-Verlag, Berlin, 1998.

[55] D. Mange, A. Stauffer, and G. Tempesti. Embryonics: A Microscopic View of the Cellular Architecture. In M. Sipper, D. Mange, and A. Pérez-Uribe, editors, Evolvable Systems: From Biology to Hardware, volume 1478 of Lecture Notes in Computer Science, pages 185-195. Springer-Verlag, Berlin, 1998. 
[56] D. Mange, A. Stauffer, and G. Tempesti. Multiplexer-Based Cells. In D. Mange and M. Tomassini, editors, Bio-Inspired Computing Machines: Toward Novel Computational Architectures, pages 121-165. Presses Polytechniques et Universitaires Romandes, Lausanne, Switzerland, 1998.

[57] D. Mange, A. Stauffer, and G. Tempesti. Self-Repairing Molecules and Cells. In D. Mange and M. Tomassini, editors, Bio-Inspired Computing Machines: Toward Novel Computational Architectures, pages 217-267. Presses Polytechniques et Universitaires Romandes, Lausanne, Switzerland, 1998.

[58] D. Mange, A. Stauffer, G. Tempesti, and C. Teuscher. Dispositif électronique à affichage électro-optique commandé par des circuits logiques programmables, March 2001. European patent, No 01201221.7.

[59] D. Mange, A. Stauffer, G. Tempesti, and C. Teuscher. From Embryonics to POEtic Machines. In J. Mira and A. Prieto, editors, Bio-Inspired Applications of Connectionism, volume 2085 of Lecture Notes in Computer Science, Part II, pages 1-13. Springer-Verlag, Berlin, June 2001.

[60] P. Marchal and P. Nussbaum. Demultiplexer-Based Cells. In D. Mange and M. Tomassini, editors, Bio-Inspired Computing Machines: Toward Novel Computational Architectures, pages 167-182. Presses Polytechniques et Universitaires Romandes, Lausanne, Switzerland, 1998.

[61] P. Marchal, P. Nussbaum, C. Piguet, S. Duran, D. Mange, E. Sanchez, A. Stauffer, and G. Tempesti. Embryonics: The Birth of Synthetic Life. In E. Sanchez and M. Tomassini, editors, Towards Evolvable Hardware, pages 166-196. SpringerVerlag, Berlin, 1996.

[62] P. Marchal, A. Tisserand, P. Nussbaum, B. Girau, and H. F. Restrepo. Array processing: A massively parallel one-chip architecture. In Proceedings of the Seventh International Conference on Microelectronics for Neural, Fuzzy, and Bio-Inspired Systems, pages 187-193, Granada, Spain, April 1999.

[63] E. J. McCluskey. Logic Design Principles with Emphasis on Testable Semicustom Circuits. Prentice-Hall, Englewood Cliffs, New Jersey, 1986.

[64] M. L. Minsky. Computation: Finite and Infinite Machines. Prentice-Hall, Englewood Cliffs, New Jersey, 1967.

[65] V. Mischler. Environnement de développment pour cellules MICTREE. Technical report, École Polytechnique Fédérale de Lausanne, Lausanne, Switzerland, February 2000.

[66] K. Misra. Reliability Analysis and Prediction. Elsevier, Amsterdam, 1992.

[67] M. Mitchell. An Introduction to Genetic Algorithms. MIT Press, Cambridge, MA, 1996. 
[68] K. Morita and K. Imai. Self-reproduction in reversible cellular space. In Theoretical Computer Science, volume 168, pages 337-366, 1996.

[69] K. Morita and K. Imai. A simple self-reproduction space automaton with shape encoding mechanism. In C. Langton and T. Shimohara, editors, Artificial Life V: Proceedings of the Fifth International Workshop on the Synthesis and Simulation of Living Systems, Cambridge, MA, 1997. MIT Press.

[70] G. García Márquez. El cataclismo de damocles. Conferencia Ixtapa, Mexico, 1986.

[71] F. Nourai. A Universal Four-State Cellular Computer. IEEE Transactions on Computers, c-24(8):766-776, August 1975.

[72] P. Nussbaum, B. Girau, and A. Tisserand. Field Programmable Processor Arrays. In M. Sipper, D. Mange, and A. Pérez-Uribe, editors, Evolvable Systems: From Biology to Hardware, volume 1478 of Lecture Notes in Computer Science, pages 311-322. Springer-Verlag, Berlin, 1998.

[73] C. Ortega, A.M. Tyrrell, D. Mange, A. Stauffer, and G. Tempesti. Reliability Analysis of a Self-Repairing Embryonic Machine. In Proceedings of the Euromicro's Symposium on Digital Systems Design, pages 356-361, Maastricht, The Nertherlands, September 2000.

[74] C. Ortega-Sánchez and A. Tyrrell. Reliability Analysis in Self-Repairing Embryonics Systems. In A. Stoica, D. Keymeulen, and J. Lohn, editors, Proceedings of the First NASA/DoD Workshop on Evolvable Hardware, pages 120-128. IEEE Computer Society, Los Alamitos, CA, July 1999.

[75] C. A. Ortega-Sánchez. Embryonics: A Bio-Inspired Fault-Tolerant Multicellular System. PhD thesis, The University of York, York, UK, May 2000.

[76] A. Perez-Uribe. Artificial Neural Networks: Algorithms and Hardware Implementation. In D. Mange and M. Tomassini, editors, Bio-Inspired Computing Machines: Toward Novel Computational Architectures, pages 289-316. Presses Polytechniques et Universitaires Romandes, Lausanne, Switzerland, 1998.

[77] A. Perez-Uribe and E. Sanchez. FPGA Implementation of an Adaptable-Size Neural Network. In International Conference on Artificial Neural Networks, Bochum, Germany, July 1996.

[78] A. Perez-Uribe and E. Sanchez. The FAST Architecture: A Neural Network with Flexible Adaptable-Size Topology. In Proceedings of the 5th International Conference on Microelectronics for Neural Networks and Fuzzy Systems, pages 337-340, February 1996.

[79] J.-Y. Perrier, M. Sipper, and J. Zahnd. Toward a Viable, Self-Reproducing Universal Computer. Physica D, 97:335-352, 1996. 
[80] U. Pesavento. An implementation of von Neumann's Self-Reproducing Machine. Artificial Life, 2(4):337-354, 1995.

[81] P. Prusinkiewicz and A. Lindenmayer. The Algorithmic Beauty of Plants. SpringerVerlag, Ney York, 1990.

[82] M. Rabii. Autotest des cellules artificielles MICROTREE. Technical report, École Polytechnique Fédérale de Lausanne, Laboratoire de systèmes logiques, Lausanne, Switzerland, September 1997.

[83] T. S. Ray. An Approach to the Synthesis of Life. In C. Langton, C. Taylor, J. D. Farmer, and S. Rasmussen, editors, Artificial Life II, volume XI of SFI Studies in the Sciences of Complexity, pages 371-408. Addisson-Wesley, Reading, MA, 1992.

[84] J. A. Reggia, S. L. Armentrout, H.-H. Chou, and Y. Peng. Simple Systems that Exhibit Self-Directed Replication. Science, 259:1282-1287, February 1993.

[85] H. F. Restrepo and D. Mange. Reconfigurable Computing. Experiences and Perspectives, chapter MICTREE: A Bio-Inspired FPGA for Embryonic Applications, pages 152-167. Fundaçao de Ensino Eurípides Soares da Rocha, Marília, SP, Brazil, August 2000.

[86] H. F. Restrepo and D. Mange. A 2-by-n Hybrid Cellular Automaton Implementation Using a Bio-Inspired FPGA. In J. Mira and A. Prieto, editors, Bio-Inspired Applications of Connectionism, volume 2085 of Lecture Notes in Computer Science, Part II, pages 39-46. Springer-Verlag, Berlin, June 2001.

[87] H. F. Restrepo and D. Mange. An Embryonic Implementation of a Self-Replicating Universal Turing Machine. In Y. Liu, K. Tanaka, M. Iwata, T. Higuchi, and M. Yasunaga, editors, Evolvable Systems: From Biology to Hardware, volume 2210 of Lecture Notes in Computer Science, pages 74-87. Springer-Verlag, Berlin, October 2001.

[88] H. F. Restrepo, D. Mange, and E. Caidedo. Implementación multicelular de una máquina universal de Turing auto-replicativa. In J. A. Hernández, editor, Proceedings of the International Congress on Computational Iintelligence, pages 67-72, Medellín, Colombia, August 2001.

[89] H. F. Restrepo, D. Mange, and E. Caidedo. MICTREE: Una FPGA reconfigurable bio-inspirada para la implementación de organismos artificiales. In J. A. Hernández, editor, Proceedings of the International Congress on Computational Iintelligence, pages 73-78, Medellín, Colombia, August 2001.

[90] H. F. Restrepo, D. Mange, and M. Sipper. A Self-Replicating Universal Turing Machine: From von Neumann's Dream to New Embryonic Circuits. In M. A. Bedau, J. S. McCaskill, N. H. Packard, and S. Rasmussen, editors, Seventh International Conference on Artificial Life, pages 3-12. MIT Press, Cambridge, August 2000. 
[91] E. Sanchez, D. Mange, M. Sipper, M. Tomassini, A. Pérez-Uribe, and A. Stauffer. Phylogeny, Ontogeny, and Epigenesis: Three Sources of Biological Inspiration for Softening Hardware. In T. Higuchi, M. Iwata, and W. Liu, editors, Evolvable Systems: From Biology to Hardware, Lecture Notes in Computer Science, pages 35-54. Springer-Verlag, Berlin, 1996.

[92] M. Sipper. Fifty Years of Research on Self-Replication: An Overview. Artificial Life, 4(3):237-257, 1998.

[93] M. Sipper, D. Mange, and A. Stauffer. Ontogenetic Hardware. BioSystems, 44(3):193-207, 1997.

[94] M. Sipper, E. Sanchez, D. Mange, M. Tomassini, A. Pérez-Uribe, and A. Stauffer. A Phylogenetic, Ontogenetic, and Epigenetic View of Bio-Inspired Hardware Systems. IEEE Transactions on Evolutionary Computation, 1(1):83-97, April 1997.

[95] M. Sipper, E. Sanchez, D. Mange, M. Tomassini, A. Perez-Uribe, and A. Stauffer. An Introduction to Bio-Inspired Machines. In D. Mange and M. Tomassini, editors, Bio-Inspired Computing Machines: Toward Novel Computational Architectures, pages 183-216. Presses Polytechniques et Universitaires Romandes, Lausanne, Switzerland, 1998.

[96] A. Stauffer, D. Mange, M. Goeke, D. Madon, G. Tempesti, S. Durand, P. Marchal, and C. Piguet. MICROTREE: Towards a Binary Decision Machine-Based FPGA with Biological-like Properties. In Proceedings of the International Workshop on Logic and Architecture Synthesis, pages 103-112, Grenoble, France, December 1996.

[97] A. Stauffer, D. Mange, E. Sanchez, G. Tempesti, S. Durand, P. Marchal, and C. Piguet. Embryonics: Towards New Design Methodologies for Circuits with Biological-like Properties. In Proceedings of the International Workshop on Logic and Architecture Synthesis, pages 299-306, Grenoble, France, December 1995.

[98] A. Stauffer, D. Mange, G. Tempesti, and C. Teuscher. BioWatch: A Giant Electronic Bio-Inspired Watch. In D. Keymeulen, A. Stoica, J. Lohn, and R. Zebulum, editors, Proceedings of the Third NASA/DoD Workshop on Evolvable Hardware, pages 185-192. IEEE Computer Society, Los Alamitos, CA, July 2001.

[99] A. Stauffer and M. Sipper. On the Relationship Between Cellular Automata and L-systems. Physica D, 116(1-2):71-80, 1998.

[100] A. Stauffer and M. Sipper. Externaly Controllable and Destructible SelfReplicating Loops. In J. Kelemen and P. Sosík, editors, Sixth European Conference on Artificial Life, volume 2159 of Lecture Notes in Artificial Intelligence, pages 282-291. Springer-Verlag, Prague, Czech Republic, 2001. 
[101] G. Tempesti. A New Self-Reproducing Cellular Automaton Capable of Construction and Computation. In F. Morán, A. Moreno, J. J. Merelo, and P. Chacón, editors, Third European Conference on Artificial Life, volume 929 of Lecture Notes in Computer Science, pages 555-563. Springer-Verlag, Heidelberg, 1995.

[102] G. Tempesti. A Self-Repairing Multiplexer-Based FPGA Inspired by Biological Processes. PhD thesis, Swiss Federal Institute of Technology, Lausanne, Switzerland, 1998.

[103] G. Tempesti, D. Mange, and A. Stauffer. A robust multiplexer-based fpga inspired by biological systems. Journal of Systems Architecture, 43(10):719-733, 1997.

[104] G. Tempesti, D. Mange, and A. Stauffer. Self-Replicating and Self-Repairing Multicellular Automata. Artificial Life, 4(3):259-282, 1998.

[105] C. Teuscher, D. Mange, A. Stauffer, and G. Tempesti. Bio-Inspired Computing Tissues: Towards Machines that Evolve, Grow, and Learn. In Fourth International Workshop on Information Processing in Cells and Tissues, pages 153-164, Leuven, Belgium, August 2001.

[106] T. Toffoli and N. Margolus. Cellular Automata Machines. MIT Press, Cambridge, MA, 1987.

[107] B. A. Trakhtenbrot. Comparing the Church and Turing Approaches: Two Prophetical Messages. In R. Herken, editor, The Universal Turing Machine a Half Century Survey, pages 557-582. Springer-Verlag, Wien, second edition, 1995.

[108] S. Trimberger, editor. Field-Programmable Gate Array Technology. Kluwer Academic Publishers, Boston, 1994.

[109] A. M. Turing. On Computable Numbers, with an Application to the Entscheidungsproblem. Proceedings of the London Math. Soc., 42:230-265, 1936.

[110] J. Villasenor and W. H. Mangione-Smith. Configurable computing. Scientific American, 276(6):54-59, June 1997.

[111] J. von Neumann. The Theory of Self-Reproducing Automata. University of Illinois Press, Urbana, Illinois, 1966. Edited and completed by A. W. Burks.

[112] H. Wang. A Variant to Turing's Theory of Computing Machines. Journal of the ACM, IV:63-92, 1957.

[113] P. H. Winston. Artificial Intelligence. Addison-Wesley, Reading, MA, third edition, 1992.

[114] N. Wirth. Programming in MODULA-2. Springer-Verlag, Berlin, 1983.

[115] D. Witting. Système de test pour cellules artificielles MICTREE. Technical report, École Polytechnique Fédérale de Lausanne, Laboratoire de systèmes logiques, Lausanne, Switzerland, June 2000. 
[116] B. Wittwer. Un simulateur pour réseaux de cellules MICTREE: Documentation du projet. Technical report, École Polytechnique Fédérale de Lausanne, Laboratoire de systèmes logiques, Lausanne, Switzerland, June 2000.

[117] B. Wittwer. Un simulateur pour réseaux de cellules MICTREE: Manuel de l'utilisateur. Technical report, École Polytechnique Fédérale de Lausanne, Laboratoire de systèmes logiques, Lausanne, Switzerland, June 2000.

[118] S. Wolfram. Theory and Applications of Cellular Automata. World Scientific, Singapore, 1986.

[119] S. Wolfram. Cellular Automata and Complexity. Addison-Wesley, Reading, MA, 1994. 


\section{Curriculum vitae}

\section{Héctor Fabio Restrepo}

Logic Systems Laboratory

EPFL-DI-LSL, INN Ecublens

CH-1015 Lausanne

Switzerland

Citizenship: Colombian
Phone: +41-21-693 6714 (W)

+41-21-626 $5327(\mathrm{H})$

Fax: +41-21-693 3705

Email:restrepo@1slsun.epfl.ch

URL:http://lslwww.epfl.ch

\section{Education}

Current Ph. D. Candidate in Computer Science, Swiss Federal Institute of Technology (EPFL), Lausanne, Switzerland.

May/98 Master Degree in Electrical Engineering, Universidad del Valle, Cali, Colombia.

Dec/96 Master Degree in Computer Science, EPFL, Lausanne, Switzerland

Sep/91 Electrical engineer, Universidad del Valle, Cali, Colombia.

\section{Professional experience}

Apr/96-Apr/97 Fellowship Holder: Training at the Logic Systems Laboratory, EPFL under the direction of professors Eduardo Sanchez and Daniel Mange

Oct/93-Mar/96 Fellowship Holder: Training at the Industrial Computer Science Laboratory, EPFL under al direction of professor Henri Nussbaumer.

Oct/92-Jun/93 Assistant professor, Electrical Engineering Department, Universidad del Valle.

Oct/91-Sep/92 Research/Teaching assistant, Electrical Engineering Department, Universidad del Valle.

Software Skills: $\quad$ Windows administration, Unix administration C, C++, Java, HTML, Pascal, Assembly, VHDL

Software Packages: CVS, ModelSim, Leonardo, Synopsys, Symplify, ViewLogic, Xilinx Alliance

Languages: $\quad$ Spanish (native language)

French (fluent)

English (fluent) 


\section{List of Publications}

$\approx$ H. F. Restrepo, D. Mange. An Embryonics Implementation of a Self-Replicating Universal Turing Machine. In Evolvable Systems: From Biology To Hardware, volume 2210 of Lecture Notes in Computer Science, pages 74-87. Springer-Verlag, Berlin, October 2001.

$\Rightarrow$ H. F. Restrepo, D. Mange, and E. Caicedo. Implementación multicelular de una máquina universal de Turing auto replicativa. In Proceedings of the International Congress on Computational Intelligence, Medellin, Colombia, August 2001.

$\approx$ H. F. Restrepo, D. Mange, and E. Caicedo. MICTREE: Una FPGA reconfigurable para la implementación de organismos artificiales en Embriónica. In Proceedings of the International Congress on Computational Intelligence, Medellin, Colombia, August 2001.

$\Rightarrow$ H. F. Restrepo, D. Mange. A 2-by-n Hybrid Cellular Automaton Implementation Using a Bio-Inspired FPGA. In J. Mira and A. Prieto, editors, Bio-Inspired Applications of Connectionism, volume 2085 of Lecture Notes in Computer Science, PartII, pages 39-46. Springer-Verlag, Berlin, June 2001.

$\Rightarrow$ J.-L. Beuchat, J.-O. Haenni, H.-F. Restrepo, C. Teuscher, F. Gomez, E. Sanchez. Approches Matérielles et Logicielles de l'Algorithme de Chiffrement IDEA. Technique et Science Informatiques, 2001. To appear.

$\Rightarrow$ H. F. Restrepo, C. A. Peña, A. Perez-Uribe. Hacia el desarrollo de nuevas máquinas computacionales: lecciones que aprendemos de la naturaleza. In Revista Energía y Computación, volume IX(2):40-47, Universidad del Valle, 2001.

$\Rightarrow$ F. J. Gómez, G. Galeano, H. F. Restrepo, J-O. Haenni, C. Teuscher, E. Sanchez. Labomat3: Un entorno completo para el aprendizaje de técnicas de codiseño utilizando una plataforma reconfigurable. IV Congreso de Tecnologías Aplicadas a la Enseñanza de la Electrónica, volume II, pp. 549-552. Barcelona, Spain. Septiembre 2000 .

$\Rightarrow$ H. F. Restrepo, D. Mange. Reconfigurable Computing Experiences and Perspectives, chapter MICTREE: A Bio-Inspired FPGA for Embryonic Applications, pages 152-167. Fundaçao de Ensino Eurípides Soares da Rocha, Marília, S.P., Brazil, August 2000 .

$\Rightarrow$ H. F. Restrepo, D. Mange, M. Sipper. A Self-Replicating Universal Turing Machine: From von Neumann's Dream to New Embryonic Circuits. In Proceedings of the Seventh International Conference on the Simulation and Synthesis of Living Systems (Artificial Life VII). Portland, Oregon, USA, August 2000.

$\Rightarrow$ J-L. Beuchat, C. Teuscher, F. Gómez, H. F. Restrepo, E. Sanchez. Une comparaison entre quelques implantations logicielles et matérielles de l'algorithme de chiffrement IDEA. 6-ème Symposium sur les Architectures Nouvelles de Machines, Rennes, France, Juin 2000. 
$\Rightarrow$ H. F. Restrepo, F. J. Gómez, C. Teuscher, J.-O. Haenni, E. Sanchez. Una Plataforma Reconfigurable para la Enseñanza de Sistemas Lógicos. Proceedings of the Interamerican Conference on Engineering and Technology Education Conference, Cincinnati, Ohio, June 2000.

$\Rightarrow$ H. F. Restrepo, R. Hoffman, A. Perez, C. Teuscher, E. Sanchez. A Networked FPGA-Based Hardware Implementation of a Neural Network Application. IEEE Symposium on Field-Programmable Custom Computing Machines, pages 337-338, Napa Valley, CA, April 2000.

$\approx$ J.-O. Haenni, C. Teuscher, F. J. Gómez, H. F. Restrepo, E. Sanchez. A Tool for Teaching and Research on Computer architecture and Reconfigurable Systems. Proceedings of the 25th EUROMICRO Conference. Milan, Italy, September 1999.

$\Rightarrow$ E. Mosanya, C. Teuscher, H. F. Restrepo, P. Galley, E. Sanchez, CryptoBooster: A Reconfigurable and Modular Cryptographic Coprocessor. Proceedings of the 1st International Workshop in Cryptographic Hardware and Embedded Systems. Worcester, MA, USA, August 1999.

$\Rightarrow$ C. Teuscher, J.-O. Haenni, F. J. Gómez, H. F. Restrepo, E. Sanchez. Labomat 3: Une plate-forme pour l'enseignement et le prototypage d'architectures reconfigurables. 5-ème Symposium sur les Architectures Nouvelles de Machines, Sympa '5, Rennes, France, Juin 1999.

$\Rightarrow$ C. Teuscher, J.-O. Haenni, F. J. Gómez, H. F. Restrepo, E. Sanchez. Labomat 3: A Reconfigurable Platform for Academic Purposes. Proceedings of the IEEE Symposium on Field-Programmable Custom Computing Machines. Napa Valley, California, USA, April 1999.

$\Rightarrow$ P. Marchal, A. Tisserand, P. Nussbaum, B.Girau, Hector Fabio Restrepo. Array Processing: A Massively Parallel One-Chip Architecture. In Proceedings of the VII International Conference on Microelectronics for Neural, Fuzzy and Bio-Inspired Systems, MicroNeuro '99, Granada, Spain, April 7-9, 1999.

$\Rightarrow$ W. Pineda, H. F. Restrepo. Lenguajes y metodologías de programación. Innovación y Ciencia - La revolución de la informática, Vol. V-1, pp. 40-53, 1996.

$\Rightarrow$ G. Berthet, P. Castori, P. Pleinevaux, F. Restrepo, K. Vijayanada, F. Vamparys. Interoperability testing: Experience with MAP and CNMA. In Proceedings of the Thirteenth IASTED International Conference, IGLS Austria, February, 1995.

$\approx$ H. F. Restrepo, W. Pineda. Historia de los Computadores. Revista Energía y Computación, volume I(4), pages. 43-47, 1992. 